\title{
Laser-Carburisierung von Eisen und Edelstahl
}

\author{
Dissertation \\ zur Erlangung des Doktorgrades \\ der Mathematisch-Naturwissenschaftlichen Fakultäten \\ der Georg-August-Universität zu Göttingen
}

vorgelegt von

Michael Kahle

aus Göttingen

Göttingen 2005 
D7

Referent:

Koreferent:

Tag der mündlichen Prüfung: 11.01.2006

Prof. Dr. Peter Schaaf

Prof. Dr. Herbert C. Freyhardt 
„Es ist falsch anzunehmen, es sei Aufgabe der Physik herauszufinden, wie die Natur ist. Physik betrifft das, was wir über die Natur sagen können." 1

Niels Bohr

\footnotetext{
${ }^{1}$ Aus: B. Herbert, Quantum Reality, Anchor Press, Garden City, New York, 1985
} 



\section{Inhaltsverzeichnis}

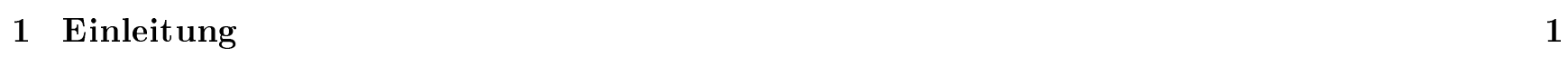

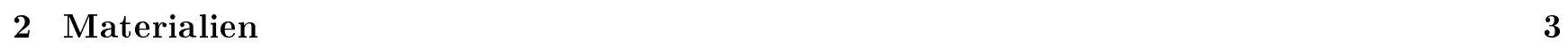

$2.1 \quad$ Das Eisen-Kohlenstoff-System . . . . . . . . . . . . . . . . . . . . 3

2.1 .1 Eigenschaften der Fe-C-Legierungen $\ldots \ldots \ldots \ldots \ldots$

2.1 .2 Eisen-Carbide . . . . . . . . . . . . . . . . . . . . 5

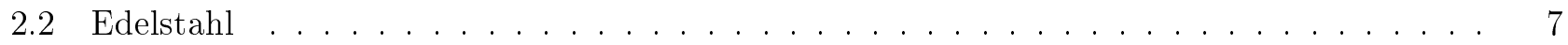

3 Experimentelle Methoden und theoretische Grundlagen 9

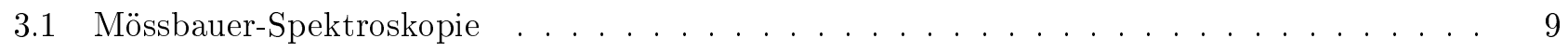

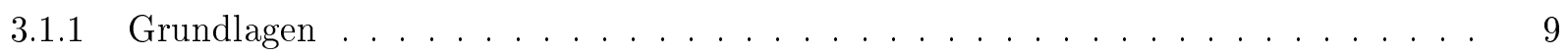

$3.1 .2 \quad$ Hyperfeinwechselwirkungen und Phasenanalyse . . . . . . . . . . . . . . 11

3.2 Röntgen-Diffraktometrie (XRD) und Kristallographie . . . . . . . . . . . . . . . . 15

$3.2 .1 \quad$ Beugungsbedingung $\ldots \ldots \ldots \ldots \ldots \ldots \ldots$

3.2 .2 Lorentz-Polarisations-Faktor $\ldots \ldots \ldots \ldots \ldots \ldots \ldots$. . . . . . . . . . . . 19

3.2 .3 Eindringtiefe und Geometrie . . . . . . . . . . . . . . . . . . 20

$3.3 \quad$ Extended X-Ray Absorption Fine-Structure (EXAFS) ～. . . . . . . . . . . . . . . . 21

3.4 Rutherford Backscattering Spectrometry (RBS) $\ldots \ldots \ldots \ldots \ldots$. . . . . . . . . . . 23

3.4 .1 Wirkungsquerschnitt . . . . . . . . . . . . . . . . . . . 24

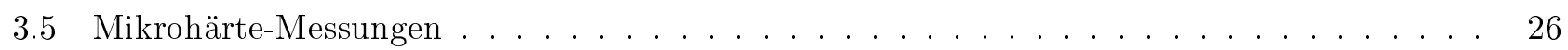

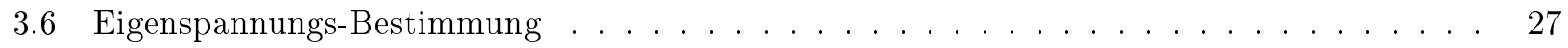

3.7 Sonstige Messmethoden $\ldots \ldots \ldots \ldots \ldots$

$3.7 .1 \quad$ Magneto-Optischer Kerr-Effekt (MOKE) $\ldots \ldots \ldots \ldots \ldots \ldots \ldots$

3.7 .2 Raman-Spektroskopie . . . . . . . . . . . . . . . . . . . . . 30

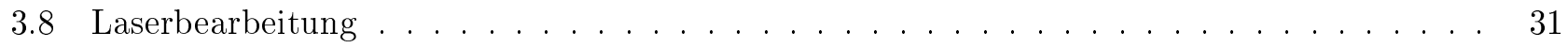

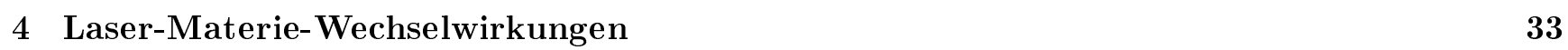

4.1 Elektronische Eigenschaften der Metalle . . . . . . . . . . . . . . . . . . . . . . . . 33

4.2 Wärmeleitungs-Gleichung . . . . . . . . . . . . . . . . . . . . . 34

4.2 .1 Abkühlrate und Temperaturgradient . . . . . . . . . . . . . . . . . 35

4.3 Numerische Berechnung der Temperatur-Profile . . . . . . . . . . . . . . . . . . . . . 36

4.4 Materialverdampfung und Plasmabildung $\ldots \ldots \ldots \ldots \ldots \ldots$

$4.4 .1 \quad$ Laser-Supported Absorption-Waves . . . . . . . . . . . . . . . . . . . . . . 37

4.5 Materietransport . . . . . . . . . . . . . . . . . . . . . . . . . . 40 
\begin{tabular}{|lll}
5 & Ergebnisse der Eisen-Bestrahlung & 43
\end{tabular}

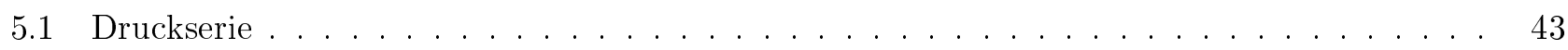

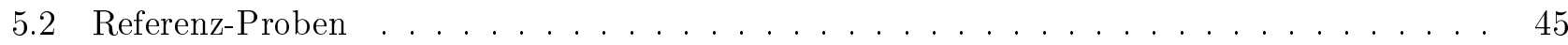

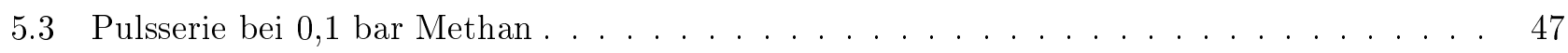

5.4 Pulsserie bei 4 bar Methan $\ldots \ldots \ldots \ldots \ldots \ldots \ldots$

5.5 Pulsserie bei 1 bar Methan $\ldots \ldots \ldots \ldots \ldots \ldots \ldots \ldots$

5.6 Vergleich der Pulsserien $\ldots \ldots \ldots \ldots \ldots \ldots \ldots \ldots \ldots$

$5.7 \quad$ Vergleich zwischen Roh- und homogenisiertem Strahl . . . . . . . . . . . . . . . . . 53

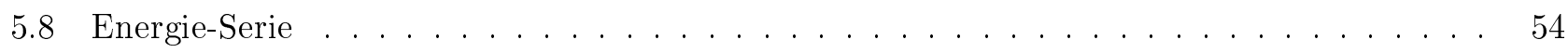

5.9 Pulsserien bei 4 bar Methan und Energiedichten von $2,5 \mathrm{Jcm}^{-2}$ bzw. $3 \mathrm{Jcm}^{-2}$. . . . . . 56

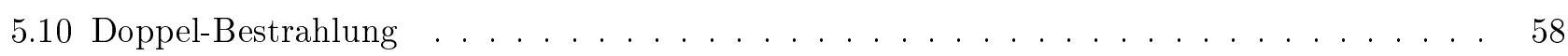

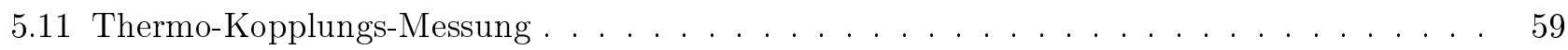

5.12 Eigenspannungs-Bestimmung $\ldots \ldots \ldots \ldots \ldots \ldots \ldots$

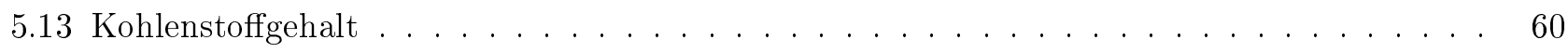

5.14 Mikrohärte-Messungen . . . . . . . . . . . . . . . . . . . . . . . . . . 61

5.15 Zusammenfassung: Phasengebiete der Puls- und Druckserie . . . . . . . . . . . . . . . . 64

\begin{tabular}{|lll}
\hline 6 & Ergebnisse der Edelstahl-Bestrahlung & 65 \\
\hline
\end{tabular}

6.1 Pulsserie bei 1 bar Methan . . . . . . . . . . . . . . . . . . . . . . . . . 66

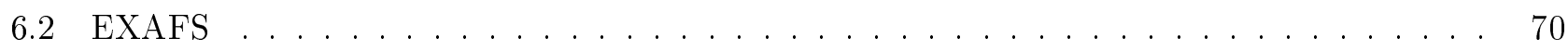

6.3 Mikrohärtemessung der Pulsserie $\ldots \ldots \ldots \ldots \ldots \ldots$. . . . . . . . . . . . 74

6.4 Bestrahlung in gemischten Gasen, Stickstoff und Ammoniak . . . . . . . . . . . . . . . . 75

6.5 Anlassen der Edelstahl-Probe $\ldots \ldots \ldots \ldots \ldots \ldots \ldots$

\begin{tabular}{|lll}
\hline & Diskussion zum Carburisierungs-Prozess & $\mathbf{7 9}$
\end{tabular}

7.1 Phasenbildungs-Mechanismus im Eisen . . . . . . . . . . . . . . . . . . . . . . 79

7.2 Phasenbildungs-Mechanismus im Edelstahl . . . . . . . . . . . . . . . . . . . 81

\begin{tabular}{|llr}
\hline 8 & Zusammenfassung & 85
\end{tabular}

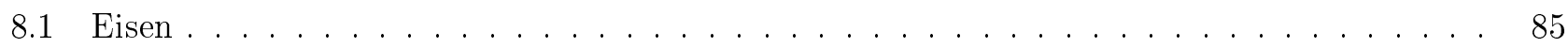

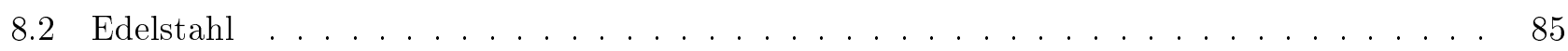

\begin{tabular}{|lrl}
\hline 9 Ausblick & 87
\end{tabular} 


\section{Kapitel 1}

\section{Einleitung}

Eisen und seine Legierungen insbesondere mit Kohlenstoff sind die am weitverbreitesten Werkstoffe, deren Herstellung und Strukturumwandlung schon seit Ende des 19. Jahrhunderts systematisch untersucht wurden. Die Prozesse des Fe-C-Legierens wurden ständig weiter entwickelt und so die Materialien gezielt an die Anforderungen angepasst. Man erkannte, dass die verbesserten Eigenschaften wie Verschleiß- und Korrosionsbeständigkeit auch dadurch erreicht werden konnten, indem man Kohlenstoff (als Ionen oder als Verbindung) lediglich in oberflächennahe Schichten des Materials einbringt, anstatt das gesamte Werkstück einem Legierungsprozess zu unterziehen. Als konventionelle Verfahren seien das Gas-Carburisieren [1,2,3], das Plasma-Carburisieren [4, 5, 6] und die Ionen-Implantation [7, 8, $9,0,10]$ erwähnt.

Bereits nach der Erfindung des Lasers wurde daran gearbeitet, dessen Vorteile und neuartige Anwendungsmöglichkeiten für die Materialbearbeitung zu nutzen. Eine industrielle Nutzung des Laser in der Materialbearbeitung ist seit ca. 30 Jahre zu verzeichnen. Der Laser vermag höchste Leistungsdichten auf einem kleinen räumlichen und zeitlichen Bereich zu erzielen, ohne das Grundmaterial oder angrenzende Oberflächenbereiche wesentlich in ihrer Beschaffenheit zu beeinflussen. Auch komplex geformte Oberflächen können schneller und kostengünstiger als durch die herkömmlichen Techniken vergütet werden. Die daraus resultierenden, vielfältigen Anwendungen sind u.a. Schneiden, Schweißen, Bohren, Umschmelzen, Härten, Legieren, Beschichten, Glasieren und Amorphisierung, Reinigen, Lithographie [11, 12,13,14. Die Ergebnisse hängen von den Größen und Änderungen der Prozessparameter wie Art der Energieeinkopplung in die Oberfläche (Strahlenergie, -Durchmesser, -Polarisation, Puls- oder Dauerbetrieb), Rastergeschwindigkeit und Eigenschaft des Materials ab, deren Kenntnis es erlaubt, die Prozessführung immer weiter zu optimieren [15, 16, 17, 18]. Ein weiteres wichtiges Anwendungsgebiet ist die Puls-Laserdeposition [11, 13], mit der beispielsweise Polymerfilme, Hochtemperatur-Supraleiter oder sehr harter, diamant-ähnlicher Kohlenstoff (DLC) hergestellt werden kann. Auf dem Gebiet der Beschleunigertechnik ist es in neuester Zeit gelungen, die starken Laser-Felder zu benutzen, um Partikel bis auf einige $\mathrm{MeV}$ zu beschleunigen [19:20].

Beim sogenannten Laser-Carburisieren wird der Kohlenstoff mit Hilfe des Lasers in das Werkstück inkorporiert. Dies geschieht dadurch, indem auf das Werkstück eine Kohlenstoff/Graphit-Schicht aufgebracht wird und der Laser (meistens im cw-Betrieb) die Oberfläche erhitzt bzw. aufschmilzt [21, 22]. Die konsequente Weiterentwicklung dieser Methode führt auf das Laser-Carburisieren in reaktiver Atmosphäre: Dabei werden Metalle in kohlenstoffreichen Gasen (CO oder $\left.\mathrm{C}_{x} \mathrm{H}_{y}\right)$ mit einem Laser bestrahlt. Im Rahmen dieser Arbeit werden Eisen und Edelstahl in Methan bearbeitet. Durch die stattfindende LaserMaterie-Wechselwirkung wird das Metall bis über den Schmelzpunkt erhitzt und der Kohlenstoff in Tiefen um $1 \mu \mathrm{m}$ inkorporiert [23]. Ein derartiges Verfahren wurde außer an Eisen 24, 25] noch an Silizium [24] und an Titan [26] angewendet. 



\section{Kapitel 2}

\section{Materialien}

Das Thema dieser Arbeit ist die Laser-Bestrahlung von Eisen- und Edelstahl-Proben in einer Methanatmosphäre. Durch die Laser-Substrat-Wechselwirkung wird Kohlenstoff von den bestrahlten Substanzen aufgenommen. Dabei entstehen je nach herrschendem Gasdruck, Laserintensität und Anzahl der LaserPulse unterschiedliche Phasen der Fe-C-Verbindungen. Dieses Kapitel liefert einen Überblick über das wichtige Fe-C-System und die auftretenden Fe-C-Verbindungen.

\subsection{Das Eisen-Kohlenstoff-System}

\subsubsection{Eigenschaften der Fe-C-Legierungen}

Die Abb 2.1 zeigt das Fe-C-Phasendiagramm [27]. Es beinhaltet sowohl interstitielle Lösungen, wie das kubisch-raumzentrierte Ferrit $(\alpha$-Fe) bzw. $(\delta$-Fe) und das kubisch-flächenzentrierte Austenit $(\gamma-\mathrm{Fe}(\mathrm{C}))$

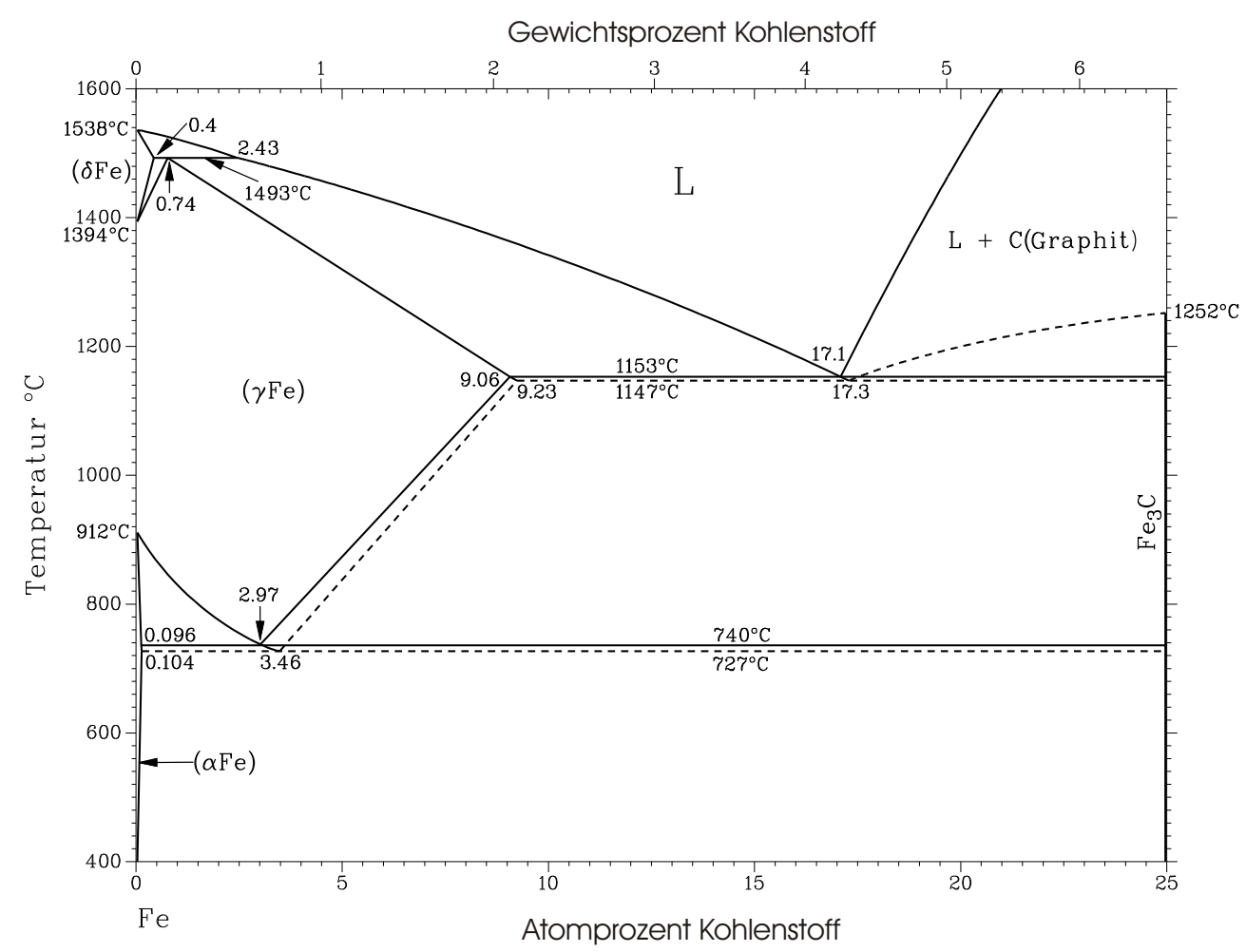

Abbildung 2.1: Fe-C-Phasendiagramm nach [27]. (Der maximale Kohlenstoffgehalt von $\gamma$-Fe(C) und $\alpha^{\prime}$-Fe variiert je nach Experimenator etwas. In Tab. 2.1 sind die Werte der Konsistenz halber nach 28 aufgeführt.)

als auch die stöchiometrische Phase $\theta-\mathrm{Fe}_{3} \mathrm{C}$ (Zementit). Im $\alpha$-Fe beträgt die maximale Löslichkeit des Kohlenstoffs 0,096 at.\% (bei $740^{\circ}$ ), in $\gamma$ - $\mathrm{Fe}(\mathrm{C})$ dagegen 9,06 at.\% (bei $1153^{\circ}$ ) [29, 30]. Übersteigt der Kohlenstoffgehalt obige Löslichkeitsgrenzen, so kann dieser als stabiles Graphit oder als metastabiles Zementit 
ausfallen; daher kann das Fe-C-System entweder als Fe-C- (-) oder als Fe-Fe 3 C-(- - -) Phasendiagramm dargestellt werden [31]. Nicht aufgeführte Phasen sind das tetragonal-raumzentrierte Martensit $(\alpha$ '-Fe) und die hexagonale Phase $\varepsilon-\mathrm{Fe}_{x} \mathrm{C}$, die eine Zusammensetzung von $2<x<6$ aufweisen kann [32, 30]. Darüber hinaus existieren noch weitere Carbide, die allesamt weniger stabil sind als das $\theta-\mathrm{Fe}_{3} \mathrm{C}$, u.a. das monokline Hägg-Carbid $\chi-\mathrm{Fe}_{5} \mathrm{C}_{2}$, das orthorhombische $\eta-\mathrm{Fe}_{2} \mathrm{C}$ bzw. $\mathrm{Fe}_{20} \mathrm{C}_{9}$, das hexagonale $\mathrm{Fe}_{2} \mathrm{C}$ und $\mathrm{Fe}_{7} \mathrm{C}_{3}$, als auch das kubische $\mathrm{Fe}_{4} \mathrm{C}$ [33, 27, 28].

Tabelle 2.1: Kristallographische Eigenschaften einiger Fe-C-Verbindungen

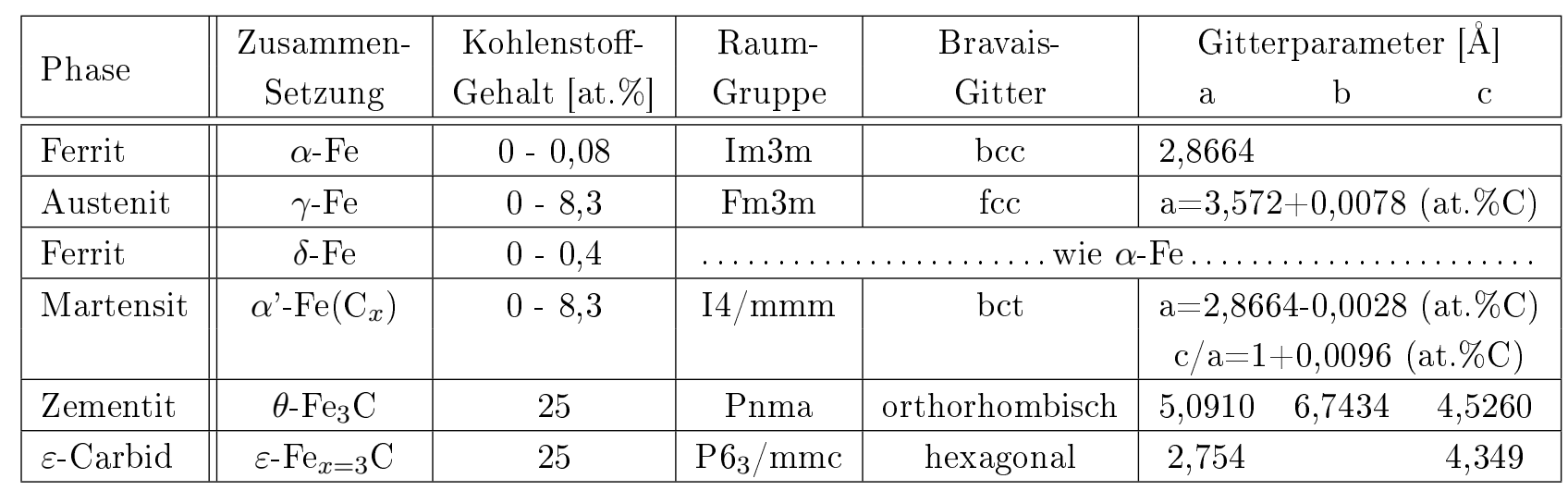

Die Tabelle 2.1 zeigt die kristallographischen Daten der wichtigsten Fe-C-Verbindungen [34, 28, 9, 27].

Tabelle 2.2: Physikalische Eigenschaften der Eisenmodifikationen und der Fe-C-Verbindungen

\begin{tabular}{|c||l|l|l|l|}
\hline $\begin{array}{c}\text { Phase }, \\
\text { Zusammensetzung }\end{array}$ & $\begin{array}{l}\text { Umwandlungspunkt } \\
\text { Schmelzpunkt }[K]\end{array}$ & Siedepunkt $[K]$ & $\rho\left[\mathrm{g} / \mathrm{cm}^{3}\right]$ & $\begin{array}{l}\text { Curie- } \\
\text { Temp. }[K]\end{array}$ \\
\hline \hline$\alpha-\mathrm{Fe}$ & $1185: \rightarrow \gamma$-Fe & & 7,86 & 1042 \\
\hline$\gamma-\mathrm{Fe}$ & $1667: \rightarrow \delta$-Fe & & 8,14 & \\
\hline$\delta-\mathrm{Fe}$ & 1811 & 2750 & 7,86 & \\
\hline$\alpha-\mathrm{FeC}_{0,03-0,08}$ & & & $7,57-7,09$ & \\
\hline$\theta-\mathrm{Fe}_{3} \mathrm{C}$ & 1525 & Zersetzung & $\approx 7,68$ & 488 \\
\hline$\varepsilon-\mathrm{Fe}_{x} \mathrm{C}$ & Umwandlung & & & $633-653$ \\
\hline
\end{tabular}

In der Tabelle 2.2 sind die wichtigsten physikalische Eigenschaften der betrachteten Fe-C-Verbindung aufgeführt [27, 34, 35, 28, 36]. Die Abb. 2.2 und 2.3 zeigen die Temperaturabhängigkeit der Löslichkeit bzw. des Diffusionskoeffizienten des Kohlenstoffs in Eisen. Unstetigkeiten kommen dadurch zustande, dass Phasenumwandlungen im Eisen auftreten. Die Diffusion von Kohlenstoff in $(\alpha$ - und $\gamma$ )-Eisen bzw. FeC-Verbindungen ist sowohl experimentell (z.B. [39,40]) als auch theoretisch 441,42] ausgiebig untersucht worden. In Abb. 2.4 sind Löslichkeitskurven in der umgänglicheren Einheit [gew.\% C] angegeben [43]. Die Löslichkeit steigt linear mit der Temperatur an. Der maximale Wert von $\approx 8,5$ gew.\% entspricht ca. 30,1 at. \% Kohlenstoff. Die Phasen-Existenzgebiete und die Phasengrenzen sind in der Abb. 2.5 dargestellt, in der die Kohlenstoffaktivität in Abhängigkeit der Temperatur aufgetragen ist. Die Aktivität $a$ ist im Allgemeinen über

$$
G=G_{0}+R T \ln a
$$

definiert [45]. $G$ ist die freie Enthalpie, $G_{0}$ die freie Standard-Enthalpie, $R$ die Gaskonstante und $T$ die Temperatur. Für eine reine Substanz ist $a=1$ und somit $G=G_{0}$. Hat man es mit Lösungen zu tun, lässt sich für das chemische Potenzial $\mu$ analog schreiben [46]:

$$
\mu=\mu_{0}+R T \ln a
$$

Die Einführung der Aktivität wird notwendig, damit das Massenwirkungsgesetz auch bei höheren Konzentrationen noch andwendbar ist, weil die Wechselwirkung der gelösten Substanzen untereinander nicht 


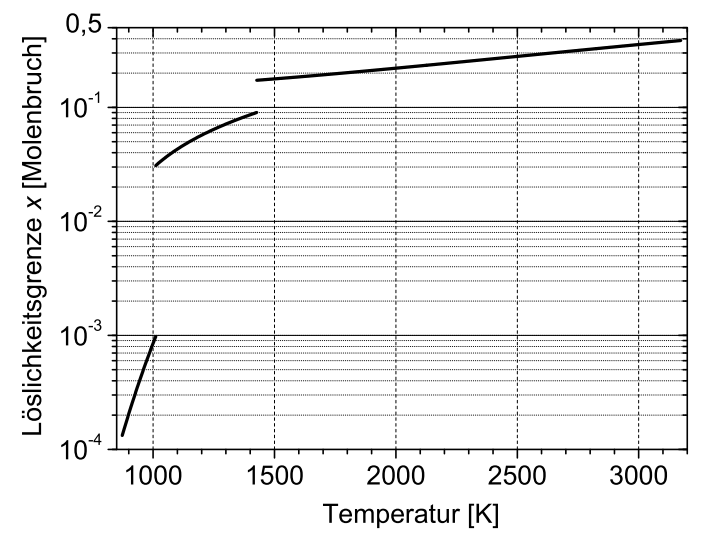

Abbildung 2.2: Löslichkeit von Kohlenstoff in Eisen (nach $[37$ ).

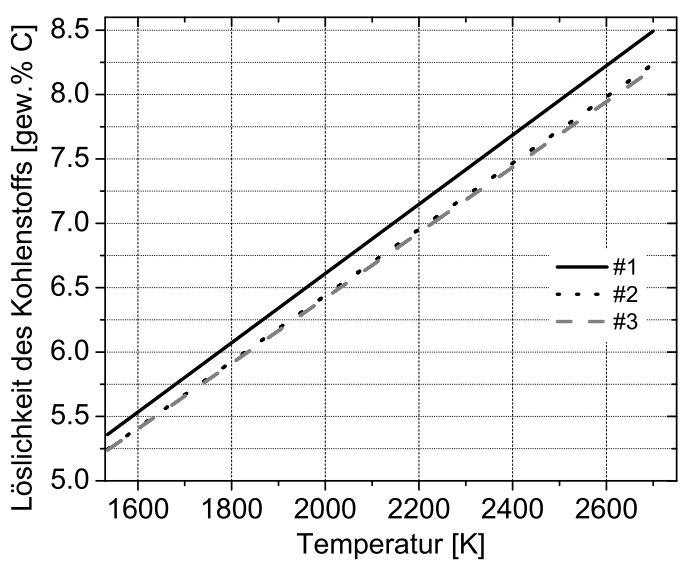

Abbildung 2.4: Löslichkeit des Kohlenstoffs in flüssigem Eisen [43] (Einheit in gew.\% C). Die Messungen mehrerer Experimentatoren (Nr. 1-3, Referenzen in [43]) zeigen, dass weitgehend Übereinstimmung der Daten herrscht.

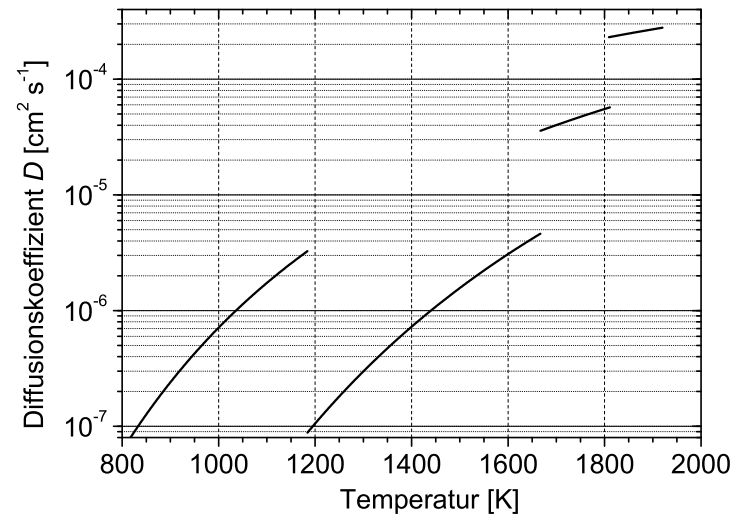

Abbildung 2.3: Diffusionskoeffizient von Kohlenstoff in Eisen (nach [37, Daten sind LiteraturDurchschnittswerte [38]).

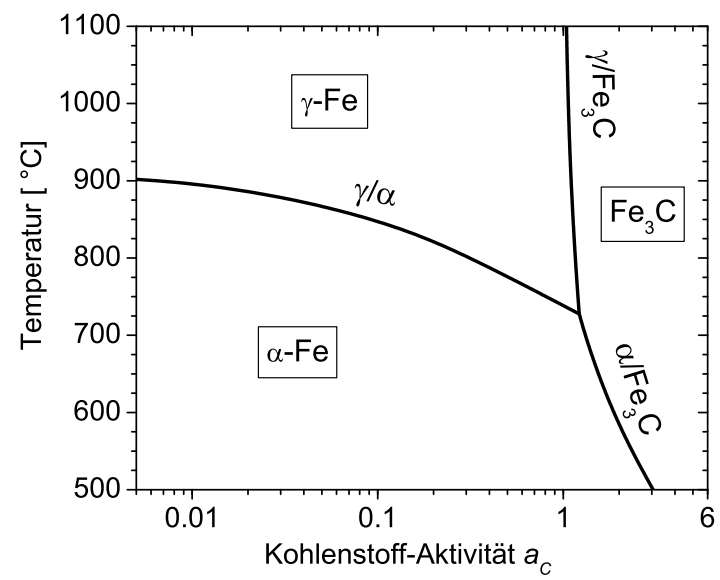

Abbildung 2.5: Temperatur-Aktivitäts-Diagramm von Kohlenstoff im Fe-C-System (Daten nach 44 reproduziert).

mehr vernächlässigt werden kann. Die Aktivität stellt damit eine effektive Konzentration dar. Die Aktivität $a$ hängt über einen Korrekturfaktor $f$, den sog. Aktivitätskoeffizienten, auf folgender Weise mit der Konzentration $c$ zusammen:

$$
a=f \cdot c
$$

Nur bei „unendlich“ verdünnten Lösungen liegen die Aktivitätskoeffizienten bei 1.

\subsubsection{Eisen-Carbide}

Die technologisch interessante Verbindung ist hier das Zementit, $\theta-\mathrm{Fe}_{3} \mathrm{C}$, dessen mechanische Eigenschaften die des Stahls in hohem Maße beeinflussen. Im folgenden werden die Strukturen und Eigenschaften der Fe-C-Verbindungen aufgeführt und die Bedingungen erläutert, unter denen sie sich ineinander umwandeln können.

\section{Das $\varepsilon$-Carbid:}

Ein wichtiges Carbid ist das $\varepsilon-\mathrm{Fe}_{x} \mathrm{C}$. Es handelt sich hierbei um eine feste Lösung von Kohlenstoff in hcp-Eisen [47], das als reine Fe-Phase bei Raumtemperatur nur oberhalb von ca. 115 kbar existent ist [48]. Die Struktur bzw. Atompositionen werden u.a. von Nagakura [49] angegeben. Die Kohlenstoffatome sitzen in der Mitte eines Okataeders, das von den umgebenen Fe-Atomen gebildet wird [32]. Ein wesentliches Merkmal ist, dass der Kohlenstoff des $\varepsilon$-Carbids in den Grenzen $2<\mathrm{x}<6$ variieren kann. 


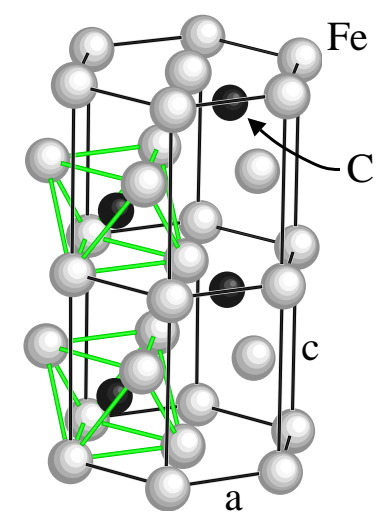

Abbildung 2.6: Hcp-Struktur des $\varepsilon-\mathrm{Fe}_{x} \mathrm{C}$. Oktaedrische Kohlenstoffpositionen sind eingezeichnet. Die dunklen, kleinen Kugeln sind die C-Atome, die großen, hellen die Fe-Atome.

\section{Das $\theta$-Carbid:}

Das $\theta-\mathrm{Fe}_{3} \mathrm{C}$ besitzt eine sehr komplexe Struktur, die u.a. von Fasiska und Jeffrey [50] analysiert wurde. Die Kristallstruktur ist zwar orthorhombisch, aber die Eisenatome bilden trigonalen Prismen, in deren Mitten die Kohlenstoffatome sitzen. Allgemein kann gesagt werden, dass alle Carbide der Zusammensetzung $\mathrm{Fe}_{3} \mathrm{C} \cdot n \mathrm{Fe}_{2} \mathrm{C}(n \in \mathbb{N})$ einen trigonal-prismatischen Aufbau zeigen [28,51]. Insbesondere zeigen $\theta-\mathrm{Fe}_{3} \mathrm{C}$ und $\chi-\mathrm{Fe}_{5} \mathrm{C}_{2}$ große $\mathrm{A} h n l i c h k e i t$, die durch fast gleiche Prismenabmessungen und Orientierung in der Einheitszelle zustandekommt. Das $\chi-\mathrm{Fe}_{5} \mathrm{C}_{2}$-Gitter ist mehr oder weniger ein verzerrtes $\theta$-Fe ${ }_{3} \mathrm{C}$-Gitter; es kann durch Scherung des $\mathrm{Fe}_{3} \mathrm{C}$ in $\mathrm{Fe}_{5} \mathrm{C}_{2}$ überführt werden [32]. In Abb. 2.7 ist der Aufbau dargestellt: Die Eisen-

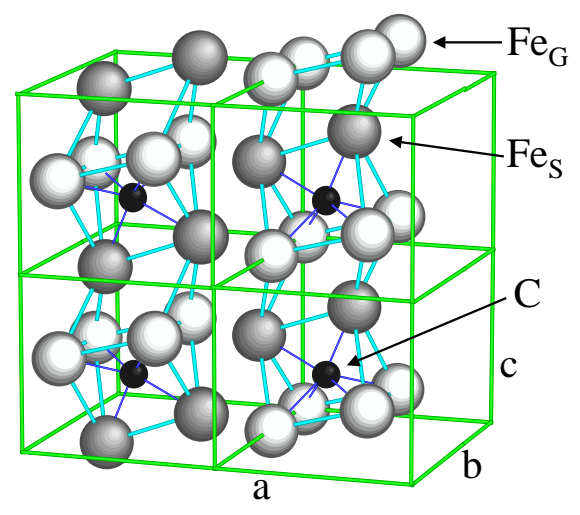

Abbildung 2. 7: Trigonal-prismatische Struktur des $\theta-\mathrm{Fe}_{3}$ C. Das Gitter stellt die Einheitszelle dar. Die inequivalenten Fe-Positionen $\mathrm{Fe}_{G}$ und $\mathrm{Fe}_{S}$ sind unterschiedlich schattiert.

atome besetzen keine gleichwertigen Position, vielmehr kann man 2 unterschiedliche Besetzungsschemata angeben: Die allgemeine Eisenposition $\mathrm{Fe}_{G}$ mit $3 \mathrm{C}$ - und $11 \mathrm{Fe}$-Atomen in nächster Nachbarschaft (NN) und die spezielle Position $\mathrm{Fe}_{S}$ mit $2 \mathrm{C}$ - und $12 \mathrm{NN}$ Fe-Atomen [33]. Der Kohlenstoffgehalt des $\theta-\mathrm{Fe}_{3} \mathrm{C}$ ist unveränderlich 25 at.\%. Darüber hinaus wurden auch Aussagen über elektronische Eigenschaften, Bindung und Stabilität durch theoretische Berechnungen gewonnen [52 [53]. Die Hyperfeinparameter weichen je nach Experimentator etwas voneinander ab. Nach Ron [30] z.B beträgt das Hyperfeinfeld von $\theta-\mathrm{Fe}_{3} \mathrm{C}$ I: $205 \mathrm{~T}$ und II: $207 \mathrm{~T}$ mit einer IS: 0,17(2) mm/s. Eine Zusammenfassung aller Mössbauerparameter von $\alpha^{\prime}$-Fe, $\theta$-Fe ${ }_{3} \mathrm{C}, \varepsilon^{\left({ }^{\prime}\right)}$ - und $\chi$-Carbide, die unter verschiedenen experimentellen Bedingungen gewonnen wurden, ist in Ron [30] gegeben.

In der Literatur wird differenziert zwischen dem hexagonal dichtest-gepackten (hcp) $\varepsilon^{\prime}$-Carbid und dem intermediären $\varepsilon$-Carbid, das strukturell zwischen hcp und monoklin einzuordnen ist [54, 55, 56]. Im Gegensatz zum $\varepsilon$-Carbid sind im hcp $\varepsilon^{\prime}$-Carbid alle kristallographisch verfügbaren Oktaederplätze gleichmäßig belegt, so dass dementsprechend nur ein Hyperfeinfeld auftritt. Sind einige Kohlenstoff-Plätze nicht besetzt, haben nicht alle Fe-Atome dieselbe Umgebungs-Konfiguration, was in unterschiedlichen Hyperfeinfeldern resultiert. Dasselbe gilt für die inequivalenten Eisen-Plätze im $\chi$ - und $\theta$-Carbid. Weiterhin gibt es ein $\mathrm{Fe}_{x} \mathrm{C}$, das eine Vorstufe des $\varepsilon$-Carbides ist [57]. Ruhl und Cohen unterscheiden darüber hinaus noch die $\varepsilon$-Phase von dem $\varepsilon$-Carbid [29]. 
Tabelle 2.3: Hyperfeinfelder der Carbide, Atompositionen(-plätze) und Curie-Temperaturen ( [3] 54 und enthaltende Ref.).

\begin{tabular}{|c|c|c|c|c|c|}
\hline Carbid & Struktur & $\mathrm{T}_{c}\left[{ }^{\circ} \mathrm{C}\right]$ & Platz & $\mathrm{H}[\mathrm{T}]$ & $\mathrm{IS}[\mathrm{mm} / \mathrm{s}]$ \\
\hline$\varepsilon^{\prime}-\mathrm{Fe}_{2,2} \mathrm{C}$ & hcp & 447 & & $173(1)$ & $+0,25(1)$ \\
\hline \multirow{3}{*}{$\varepsilon-\mathrm{Fe}_{2} \mathrm{C}$} & \multirow{3}{*}{ hcp $\rightarrow$ monoklin } & \multirow{3}{*}{377} & $\mathrm{I}$ & $170(3)$ & $+0,20(5)$ \\
\hline & & & II & $237(3)$ & 0,35 \\
\hline & & & III & $130(6)$ & 0,30 \\
\hline \multirow{3}{*}{$\chi-\mathrm{Fe}_{5} \mathrm{C}_{2}$} & \multirow{3}{*}{ monoklin } & \multirow{3}{*}{252} & I & $184(3)$ & $0,30(4)$ \\
\hline & & & II & $222(3)$ & $0,35(4)$ \\
\hline & & & III & 110 & $0,30(8)$ \\
\hline \multirow[t]{2}{*}{$\theta-\mathrm{Fe}_{3} \mathrm{C}$} & \multirow[t]{2}{*}{ orthorhombisch } & \multirow[t]{2}{*}{207} & $\mathrm{I}$ & $208(7)$ & \multirow[t]{2}{*}{$+0,29(2)$} \\
\hline & & & II & Site $\mathrm{I} \pm 5$ & \\
\hline
\end{tabular}

Im Rahmen dieser Arbeit sollen diese Feinheiten nicht ausschlaggebend sein und das $\varepsilon$-Carbid an sich betrachtet werden, welches hier einfach mit $\varepsilon-\mathrm{Fe}_{x} \mathrm{C}$ bezeichnet wird. $\mathrm{Ob}$ eines der Carbide $\mathrm{Fe}_{6} \mathrm{C}, \mathrm{Fe}_{3} \mathrm{C}$ oder $\mathrm{Fe}_{2} \mathrm{C}$ vorliegt, hängt davon ab, wieviele Oktaederplätze mit Kohlenstoff besetzt sind (s. auch [32]). In den $\varepsilon$-Carbid enthaltenden Proben sind sie in unterschiedlichen Anteilen vorhanden, die man mithilfe der Mössbauerspektren bestimmen kann. Die Stabilität der Carbide kann folgendermaßen angeordnet werden

$$
\varepsilon^{\left({ }^{\prime}\right)} \text {-Carbid }\left(\mathrm{Fe}_{2,0-2,4} \mathrm{C}\right)<\chi-\mathrm{Fe}_{5} \mathrm{C}_{2}<\mathrm{Fe}_{3} \mathrm{C}
$$

und nimmt mit steigendem Kohlenstoffgehalt ab [56 58]. In der folgenden Tab. 2.4 ist die Bildungssequenz der Carbide mit steigender Temperatur angegeben, die Temperaturen sind nur Richtwerte, da sie je nach Experimentator und Versuchsbedingungen voneinander abweichen: Die treibende Kraft, um $\theta-\mathrm{Fe}_{3} \mathrm{C} \mathrm{zu}$

Tabelle 2.4: Richtwerte für die Umwandlungstemperaturen der Carbide [59 60 61].

\begin{tabular}{|c|c|}
\hline Umwandlung & Temperatur $\left[{ }^{\circ} \mathrm{C}\right]$ \\
\hline \hline Fe-C-Cluster $/$ Martensit $\rightarrow \varepsilon$-Carbid & 200 \\
\hline$\varepsilon$-Carbid $\rightarrow \chi-\mathrm{Fe}_{5} \mathrm{C}_{3}$ & $380-400$ \\
\hline$\chi-\mathrm{Fe}_{5} \mathrm{C}_{2} \rightarrow \theta-\mathrm{Fe}_{3} \mathrm{C}$ & 560 \\
\hline$\theta-\mathrm{Fe}_{3} \mathrm{C} \rightarrow \mathrm{Fe}_{7} \mathrm{C}_{3}$ & 1400 (und $p=80 \mathrm{kbar}$ ) \\
\hline
\end{tabular}

bilden, rührt von der chemischen Bindungsenergie der Fe-C-Bindung her. $\varepsilon$-Carbid bildet sich vielmehr, um Spannungen abzubauen, wenn es aus dem $\alpha^{\prime}$-Martensit durch eine Umorientierung hervorgeht 62 63]: Es ist für den Phasenumwandlungsmechanismus aufschlussreich, auf welche Weise sich die strukturellen Umgruppierungen zwischen den verschiedenen Gitterstrukturen abspielen. Anhand der Orientierungsbeziehungen der einzelnen kristallographischen Ebenen und Atome zueinander ist es ersichtlich, wie der Übergang z.B. von $\varepsilon \rightarrow \theta$ oder $\alpha^{\left({ }^{\prime}\right)} \rightarrow \varepsilon$ abläuft. [63 64,65$]$.

\subsection{Edelstahl}

Generell werden als „rostfreie Edelstähle“ (stainless steel) Fe-Legierungen bezeichnet, die mehr als 12 gew.\% Cr enthalten [66]. Der verwendete rostfreie, austenitische Edelstahl ist eine quaternäre Legierung aus $\mathrm{Fe}, \mathrm{Cr}$, Ni und Mo. Weitere Elemente kommen aufgrund des Herstellungsprozesses in Spuren vor. Die metallurgische Bezeichnung lautet X5CrNiMo18.10.3 (Werkstoff-Nr.: 1.4401) oder gleichbedeutend AISI 316. Die Zusammensetzung ist in Tab. 2.5 angegeben: Der Edelstahl besitzt wie auch das $\gamma-\mathrm{Fe}(\mathrm{C})$ eine fcc-Gitterstruktur. Das $\gamma-\mathrm{Fe}(\mathrm{C})$ wird durch den interstitiell eingelagerten Kohlenstoff stabilisiert, so dass es auch bei Raumtemperatur vorliegen kann. Bei 1.4401 wird die fcc-Struktur des Edelstahls durch das substitutionelle Element Ni stabilisiert [66.68] (s.a. Abb. 2.8 und 2.9). Über die magnetischen 
Tabelle 2.5: Zusammensetzung des Edelstahls X5CrNiMo18.10.3 67.

\begin{tabular}{|l||c|c|c|c|c|c|c|c|}
\hline & $\mathrm{C}$ & $\mathrm{Si}$ & $\mathrm{Mn}$ & $\mathrm{P}$ & $\mathrm{S}$ & $\mathrm{Cr}$ & $\mathrm{Mo}$ & $\mathrm{Ni}$ \\
\hline gew.\% & $\leq 0,07$ & 1,0 & 2,0 & 0,045 & 0,030 & $16,5-18,5$ & $2,0-2,5$ & $10,5-13,5$ \\
\hline
\end{tabular}

Eigenschaften lässt sich sagen, dass AISI 316 antiferromagnetisch ist, dessen Néel-Temperatur bei 22,5 K liegt [69].

Der Edelstahl zeichnet sich besonders durch seine Widerstandsfähigkeit gegenüber Loch- bzw. Spaltkorrosion, hoher Verschleiffestigkeit, Zähigkeit und Streckfestigkeit aus. Das im Edelstahl enthaltene Cr bildet mit Luftsauerstoff eine passivierende Schicht aus Chrom-Oxiden, die das darunter liegende Material vor weiterer Korrosion schützt.

In den Abb. 2.8 und 2.9 sind die ternären Fe-Cr-Ni-Phasendiagramme bei $900^{\circ} \mathrm{C}$ und Raumtemperatur dargestellt, die die Komposition des X5CrNiMo18.10.3-Edelstahls zumindest in diesen drei Komponenten annähern. Das entsprechende quaternäre Phasendiagramm war nicht zugänglich. Die MolybdänKomponente wäre in diesem Fall die senkrecht zur Papierebene hinauslaufende Achse. Zu beachten sind die unterschiedlich angeordneten Komponenten-Achsen und -Gehaltsangaben (gew.\% oder at.\%). Das

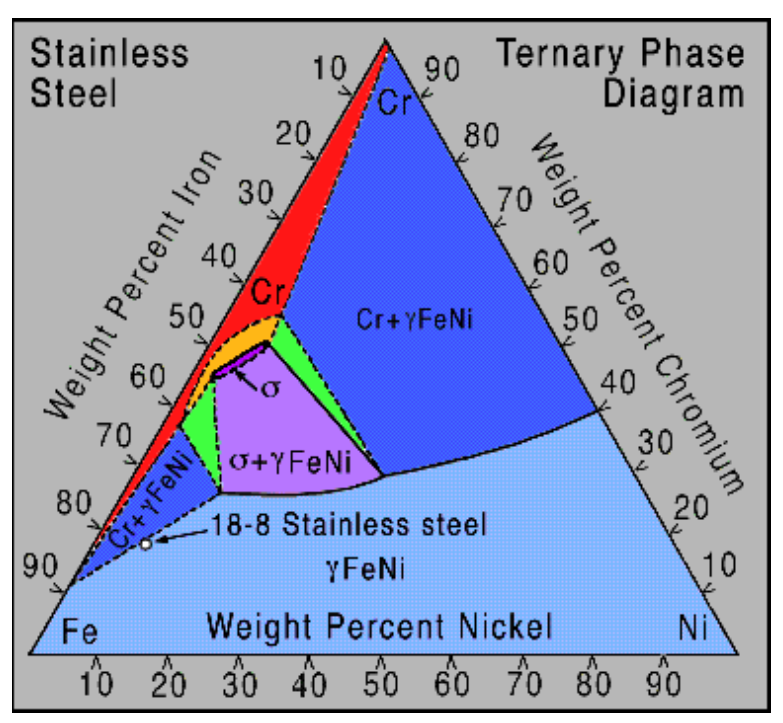

Abbildung 2.8: Fe-Cr-Ni-Phasendiagramm bei $900^{\circ} \mathrm{C}$ (nach [70]). Der weit verbreitete $18 \mathrm{Cr} 8 \mathrm{Ni}$-Edelstahl ist eingezeichnet.

Phasendiagramm in Abb. 2.9 zeigt insbesondere die Gebiete unterschiedlicher Struktur (fcc,bcc,bct).
Abbildung 2.9: Fe-Cr-Ni-Phasendiagramm bei Raumtemperatur $\left(25^{\circ} \mathrm{C}\right)$ 71. Das „X“ kennzeichnet die Zusammensetzung des $18 \overline{\mathrm{Cr}} 10 \mathrm{Ni}-\mathrm{Edelstahls} \mathrm{im} \mathrm{austenitischen} \mathrm{Gebiet.}$ Der Komponenten-Gehalt ist im Gegensatz zu denen in Abb. 2.8 in at.\% angegeben. 


\section{Kapitel 3}

\section{Experimentelle Methoden und theoretische Grundlagen}

Zur zerstörungsfreien Material- und Phasenanalyse wurden unterschiedliche Methoden angewendet, die im folgenden ausführlich erklärt werden.

\subsection{Mössbauer-Spektroskopie}

Der grundlegende Effekt ist die rückstoßffreie Kernresonanzabsorption, den Rudolf Mössbauer 1957 während seiner Doktorarbeit am Isotop ${ }^{191}$ Os entdeckte $72,73.74$. Die große Bedeutung liegt in der extrem hohen Energieauflösung, die es u.a. auch erlaubt, die chemische und kristallographische Umgebung (insbesondere deren Änderungen) des Mössbauer-Kerns zu untersuchen. Das zweifellos wichtigste MössbauerIsotop ist ${ }^{57} \mathrm{Fe}$, was den Materialwissenschaften zu einer höchst leistungsfähigen Analysemethode verholfen hat [75,76]. Seit dieser Entdeckung ist die Mössbauer-Spektroskopie sehr schnell zu einem überaus wichtigen Werkzeug in der kernphysikalischen Meßtechnik geworden. Auch in der Chemie, Biologie und Geologie findet sie einen immer größer werdenden Anwendungsbereich. Es ist eine große Anzahl an wissenschaftlichen Veröffentlichungen, Arbeiten und Büchern zu diesem Thema erschienen; als Beispiel sei auf die ausführlichen theoretischen und experimentellen Grundlagen in $777,78,79,80,81,82,83,84]$ verwiesen.

\subsubsection{Grundlagen}

Bei der Kernresonanzabsorption emittiert ein ${ }^{57} \mathrm{Fe}$-Kern der Masse $M$ ein $\gamma$-Quant der Energie $E_{\gamma}$. Unter Beachtung der Impuls- und Energieerhaltung gilt für die Rückstoßenergie $E_{R}$

$$
E_{R}=\frac{E_{\gamma}^{2}}{2 M c^{2}}=1,95 \cdot 10^{-3} \mathrm{eV}
$$

Um diesen Betrag $E_{R}$ ist die Anregungs-(Resonanz-)energie $E^{*}$ größer: $E^{*}=E_{\gamma}+E_{R}$. Da der absorbierende Kern die gleiche Rückstoßenergie $E_{R}$ aufnimmt, gilt für die Resonanzverstimmung $\Delta E$ :

$$
\Delta E=2 E_{R}=\frac{E_{\gamma}^{2}}{M c^{2}} \approx \frac{E^{* 2}}{M c^{2}}
$$

Resonanzabsorption tritt nur dann auf, wenn die Absorptions- und Emissionslinien sich überlappen, d.h. wenn zwischen $\Delta E$ und der natürlichen Linienbreite $\Gamma$ die Beziehung

$$
2 \cdot E_{R} \leq \Gamma
$$

herrscht. Ein Vergleich mit $\Gamma$

$$
\Gamma=\frac{\hbar}{\tau}=4,66 \cdot 10^{-9} \mathrm{eV}
$$

zeigt, dass $2 E_{R} \simeq 10^{6} \Gamma$ und somit bei weitem keine Resonanz vorliegt. 
Tabelle 3.1: Eigenschaften des Mössbauer-Isotops ${ }^{57} \mathrm{Fe}, 85,80,86,87$.

\begin{tabular}{|c|l|l|}
\hline \multicolumn{2}{|c|}{ Nukleare Daten } & Größe \\
\hline \hline$E_{\gamma}$ & Energie der $\gamma$-Strahlung & $14,41 \mathrm{keV}$ \\
\hline$E_{R}$ & Rückstoßenergie & $1,9563 \cdot 10^{-3} \mathrm{eV}$ \\
\hline$\Gamma$ & Natürliche Linienbreite (FWHM) & $4,66 \cdot 10^{-9} \mathrm{eV}$ \\
\hline$M_{57} \mathrm{Fe}$ & Masse von ${ }^{57} \mathrm{Fe}$ & $56,9354 \mathrm{a} . \mathrm{m} . \mathrm{u}$ \\
\hline$t_{1 / 2}\left({ }^{57} \mathrm{Fe}\right)$ & Halbwertszeit des ${ }^{57} \mathrm{Fe}$ & $97,81 \mathrm{~ns}$ \\
\hline$t_{1 / 2}\left({ }^{57} \mathrm{Co}\right)$ & Halbwertszeit des ${ }^{57} \mathrm{Co}$ & $270,9 \mathrm{~d}$ \\
\hline$\sigma_{0}$ & Max. Resonanz-Querschnitt & $2,5575 \cdot 10^{6} \mathrm{~b}$ \\
\hline$\alpha$ & Koeffizient d. inneren Konversion & 8,18 \\
\hline \multicolumn{2}{|c|}{ Natürliche Isotopenhäufigkeit } & $2,19 \%$ \\
\hline \multicolumn{2}{|c}{} \\
\hline
\end{tabular}

\section{Debye-Waller-Faktor und rückstoßfreie Emission}

Quantenmechanisch wird die Wahrscheinlichkeit, dass die Emission eines $\gamma$-Quantes rückstoßfrei erfolgt, durch den Debye-Waller-Faktor $W$ [88]

$$
f_{D}(T)=\exp \left\{-\frac{6 E_{R}}{k_{B} \Theta} \cdot\left[\frac{1}{4}+\left(\frac{T}{\Theta}\right)^{2} \int_{0}^{T / \Theta} \frac{x d x}{e^{x}-1}\right]\right\}=\exp [-2 W]
$$

gegeben. $k_{B}$ ist die Boltzmann-Konstante und $\Theta_{D}$ die Debye-Temperatur, die eine wichtige Größe im Zusammenhang mit Gitterschwingungen bzw. dem zugehörigen Phononenspektrum ist. Die Rückstoßenergie $E_{R}$ kann auch indirekt durch Phononenanregung auf den Kristall übertragen werden. Ein Vergleich von $k_{B} T$ mit $k_{B} \Theta_{D}$ gibt Aufschluss darüber, in welchem Maße der Energieübertrag an das Gitter stattfindet: Ist $T \gg \Theta_{D}$, so sind alle Schwingungsmoden des Festkörpers angeregt, d.h. die Rückstoßenergie kann auf das Gitter übertragen werden. Im umgekehrten Fall $T \ll \Theta_{D}$ ist die Wahrscheinlichkeit gering, dass $E_{R}$ in das Phononenspektrum aufgenommen wird und somit steigt $f_{D}(T)$ an. Die Debye-Temperatur bestimmt die Grenze zwischen dem niedrigen Temperaturbereich, in dem die Quantenstatistik gültig ist, und dem hohen Temperaturbereich, der durch die klassische, statistische Mechanik beschrieben wird [89]. Rechnerisch ergibt sich die Debye-Temperatur als Maximal- oder Cut-off-Frequenz $\Theta_{D}=\hbar \omega_{D} / k_{B}$ aus der sog. Debye-Näherung [90]: Die Gitterschwingungen (Phononen) im Kristall werden auf $3 N$ beschränkt, wobei $N$ die Anzahl der Atome ist. Die Debye-Frequenz $\omega_{D}$ ist dann folgendermaßen mit der PhononenZustandsdichte $Z(\omega)$ verknüpft:

$$
\int_{0}^{\omega_{D}} Z(\omega) d \omega=3 N
$$

Der Debye-Waller-Faktor $W$ ist ursprünglich aus der Theorie der Röntgenstreuung entstanden. In Bezug auf den Mössbauer-Effekt bezeichnet man $W$ Mössbauer-Lamb-Faktor. Der Grund für diese Unterscheidung sind die verschiedenen Zeit-Skalen, auf denen beide Prozesse ablaufen, denn die Lebensdauer eines Mössbauerzustandes ist länger als die Periodendauer einer Gitterschwingung, wohingegen RöntgenStreuung ein kurzzeitiger Prozess ist [83].

Das Strahlungsfeld der $\gamma$-Strahlung kann als gedämpfte Schwingung in der Form

$$
E(t)=E_{0} \cdot \exp \left(i \omega_{0} t-\Gamma / 2\right)=E_{0} \cdot \exp \left(-\left(\Gamma / 2-i \omega_{0} t\right)\right)
$$

dargestellt werden (sog. Breit-Wigner-Formel) [91]. Der Term $\Gamma / 2$ ist ein Dämpfungsterm, der über die Energieunschärfe

$$
\Gamma \cdot \tau \simeq \hbar
$$

die endliche Lebensdauer $\tau$ des Mössbauer-Zustandes mit der Linienbreite $\Gamma$ verknüpft. Das FourierSpektrum von $E(t)$ lautet dann

$$
\mathcal{F}(E(t))=F(\omega)=\frac{1}{\sqrt{2 \pi}} \int_{0}^{\infty} E(t) \cdot \exp (i \omega t) d t=\frac{1}{\sqrt{2 \pi}} \int_{0}^{\infty} E_{0} \cdot \exp \left(i\left(\omega-\omega_{0}\right) t-\Gamma / 2\right) d t
$$


Integration und Quadrieren ergibt dann die sog. Lorentzform oder Breit-Wigner-Formel (in der noch $\omega$ durch $E$ ersetzt ist) [92]:

$$
\sigma(E)=\frac{\sigma_{0} \Gamma^{2} / 4}{\left(E-E_{0}\right)^{2}+\Gamma^{2} / 4}
$$

wobei $\sigma_{0}$ der der Resonanz-Wirkungsquerschnitt ist:

$$
\sigma_{0}=\frac{\lambda^{2}}{2 \pi} \cdot \frac{2 I_{a}+1}{2 I_{g}+1} \cdot \frac{1}{1+\alpha}
$$

$I_{a}$ und $I_{g}$ sind die nuklearen Spins im angeregten bzw. Grundzustand, $\alpha$ ist der Konversions-Koeffizient des Überganges (s. Abschnitt 3.1.2.4 und $\lambda$ die Wellenlänge der Mössbauer-Strahlung.

\subsubsection{Hyperfeinwechselwirkungen und Phasenanalyse}

Aufgrund der extrem hohen Energieauflösung ist es möglich, die Hyperfeinwechselwirkung direkt zu beobachten. Die bei der Analyse der Spektren wichtigen Parameter werden im folgenden erklärt:

Die elektrostatische Wechselwirkung des Kerns mit der Ladung $Z e$ mit der umgebenen Ladung ist gegeben durch

$$
E_{\mathrm{el}}=\int \rho_{n}(\mathbf{r}) \phi(\mathbf{r}) d V,
$$

dabei sind $\rho_{n}(\mathbf{r})$ die Kernladungsdichte und $\phi(\mathbf{r})$ das Coulomb-Potenzial am Punkt $\mathbf{r}$. Das Potenzial $\phi(\mathbf{r})$ kann um $\mathbf{r}=0$ in eine Taylor-Reihe bis zur 2. Ordnung entwickelt werden:

$$
\phi(\mathbf{r})=\phi(\mathbf{0})+\left.\sum_{i=0}^{3} \frac{\partial \phi}{\partial x_{i}}\right|_{0} x_{i}+\left.\frac{1}{2} \sum_{i, j=0}^{3} \frac{\partial^{2} \phi}{\partial x_{i} \partial x_{j}}\right|_{0} x_{i} x_{j}+\mathcal{O}\left(\partial^{3} \phi / \partial x^{3}\right)
$$

In Gleichung 3.12 eingesetzt ergibt das:

$$
E_{\mathrm{el}}=\phi(\mathbf{0}) \int \rho_{n}(\mathbf{r}) d V+\left.\sum_{i=1}^{3} \frac{\partial \phi}{\partial x_{i}}\right|_{0} \cdot \int \rho_{n}(\mathbf{r}) x_{i} d V+\frac{1}{2} \sum_{i, j=3}^{3} \underbrace{\left.\frac{\partial^{2} \phi}{\partial x_{i} \partial x_{j}}\right|_{0}}_{:=\phi_{i i}} \cdot \int \rho_{n}(\mathbf{r}) x_{i} x_{j} d V
$$

Der $3 \times 3$-Tensor $\phi_{i j}$ kann durch eine Hauptachsen-Transformation diagonalisiert werden [80], so dass nur Diagonal-Elemente des Tensors $\phi_{i i}$, der auch als Elektrischer Feld-Gradient Tensor (EFG) bezeichnet wird, vorkommen. Nach Umordnen des 3. Terms erhält man:

$$
E^{(3)}=\frac{1}{2} \sum_{i=1}^{3} \phi_{i i} \cdot \int \rho_{n}(\mathbf{r}) x_{i}^{2} d V=\frac{1}{2} \sum_{i=1}^{3} \phi_{i i} \cdot \int \rho_{n}(\mathbf{r})\left(x_{i}^{2}-\frac{r^{2}}{3}\right) d V+\frac{1}{6} \sum_{i=1}^{3} \phi_{i i} \int \rho(\mathbf{r}) r^{2} d V
$$

Am Kernort gilt die Poisson-Gleichung

$$
\left(\sum_{i=3}^{3} \phi_{i i}\right)_{0}=4 \pi e|\Psi(\mathbf{0})|^{2}
$$

wobei $e|\Psi(\mathbf{0})|^{2}$ die Ladungdichte des s-Elekrons am Kernort bezeichnet [93. Somit erhält man für den elektrischen Wechselwirkungsterm $E_{\mathrm{el}}$ :

$$
E_{\mathrm{el}}=\frac{2}{3} \pi e|\Psi(0)|^{2} \cdot \int \rho_{n}(\mathbf{r}) r^{2} d V+\frac{1}{2} \sum_{i=1}^{3} \phi_{i i} \cdot \int \rho_{n}(\mathbf{r})\left(x_{i}^{2}-\frac{r^{2}}{3}\right) d V:=E_{I S}+E_{Q S}
$$

Der erste Term $E_{I S}$ ist die Elektrische Monopol-Wechselwirkung und ist gleichbedeutend mit dem Isomerieverschiebung $I S$; der letzte Term beschreibt die elektrische Quadrupol-Wechselwirkung $Q S$. 


\subsubsection{Elektrische Monopolwechselwirkung und Isomerieverschiebung}

Für den Erwartungswert des Quadrates des Kernradius in angeregten oder Grundzustand gilt:

$$
\int \rho_{n}(\mathbf{r}) r^{2} d V=\left\langle r^{2}\right\rangle_{a, g} \cdot Z e
$$

Der Energieunterschied des nuklearen Übergangs zwischen Grund- und angeregtem Zustand beträgt damit für den Quell-Kern

$$
\Delta E_{I S}^{(Q)}=E_{Q}-E_{0}=\frac{2}{3} \pi Z e^{2}\left|\Psi_{Q}(0)\right|^{2}\left(\left\langle r^{2}\right\rangle_{a}-\left\langle r^{2}\right\rangle_{g}\right)
$$

bzw. für den Absorber-Kern (s. Abb. 3.1

$$
\Delta E_{I S}^{(A)}=E_{A}-E_{0}=\frac{2}{3} \pi Z e^{2}\left|\Psi_{A}(0)\right|^{2}\left(\left\langle r^{2}\right\rangle_{a}-\left\langle r^{2}\right\rangle_{g}\right)
$$

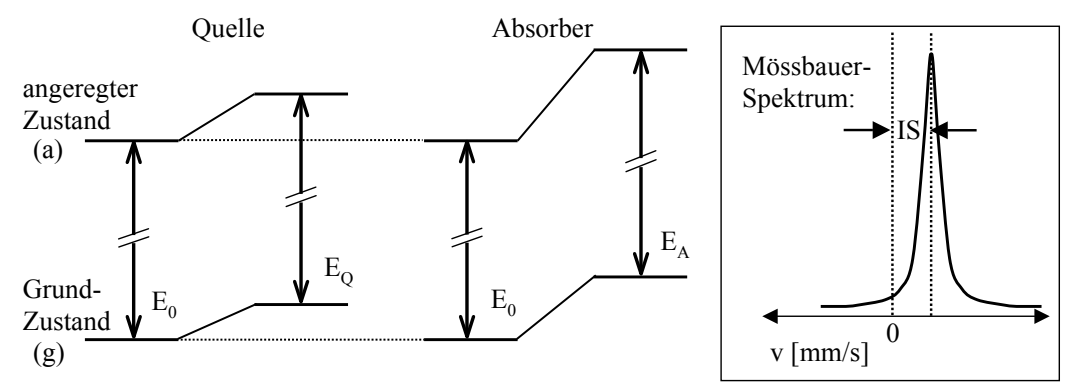

Abbildung 3.1: Elektrische Monopolwechselwirkung und Isomerieshift IS.

Die Differenz der Übergangsenergien zwischen Quell- und Absorber-Kern ist der Isomerie-Shift IS

$$
I S:=\Delta E_{I S}^{(Q)}-\Delta E_{I S}^{(A)}=\frac{2}{3} \pi Z e^{2}\left|\Psi_{Q}(0)-\Psi_{A}(0)\right|\left(\left\langle r^{2}\right\rangle_{a}-\left\langle r^{2}\right\rangle_{g}\right)
$$

\subsubsection{Elektrische Quadrupol-Wechselwirkung und Quadrupolaufspaltung}

In den $E_{Q S^{-}}$Term der Gl. 3.17 kann man das Quadrupolmoment Q gemäß

$$
e Q_{i i}=e Q=\int \rho_{n}(\mathbf{r})\left(3 x_{i}^{2}-r^{2}\right)=\sqrt{\frac{16 \pi}{5}} \rho_{n}(\mathbf{r}) r^{2} \mathbf{Y}_{2}^{0} d V
$$

einführen [94 93]. Y Y ist eine Kugelfächenfunktion. Man kann jede Ladungsverteilung nach Multipolmomenten entwickeln, die durch die Kugelfunktionen ausgedrückt werden [95]. Geht man zur Quantenmechanik über, so definiert $Q$ den Erwartungswert des Quadrupoloperators $Z r^{2} \hat{\mathbf{Y}}_{2}^{0}$ [93]:

$$
Q=\sqrt{\frac{16 \pi}{5}}\left\langle I, m_{I}=I\left|Z r^{2} \hat{\mathbf{Y}}_{2}^{0}\right| I, m_{I}=I\right\rangle=\sqrt{\frac{16 \pi}{5}} \cdot\left\langle I, m_{I}=I\left|\hat{\mathbf{Q}}_{2}^{0}\right| I, m_{I}=I\right\rangle
$$

Man definiert den Asymmetrie-Parameter $\eta$ auf folgende Weise

$$
\eta=\frac{\phi_{x x}-\phi_{y y}}{\phi_{z z}} \quad \text { mit der Beziehung: } \quad\left|\phi_{z z}\right| \geq\left|\phi_{y y}\right| \geq\left|\phi_{x x}\right| \Rightarrow 0 \leq \eta \leq 1
$$

Die Eigenschaften des EFGen sind durch $\phi_{z z}$ und $\eta$ vollständig beschrieben, da die Laplace-Gleichung erfüllt sein muss

$$
\Delta \phi=\phi_{x x}+\phi_{y y}+\phi_{z z}=0
$$

Die Werte für den Spin des ${ }^{57} \mathrm{Fe}$ liegen im Grund- bzw. angeregten Zustand bei $I_{g}=1 / 2$ und $I_{a}=3 / 2$, so dass sich für die Quadrupolaufspaltung $Q S=\Delta E_{q}$ ergibt (s. Abb. 3.2

$$
Q S:=\Delta E_{q}=\frac{e Q \phi_{z z}}{4 I(2 I-1)}\left[3 m_{I}^{2}-I(I+1)\right] \cdot \sqrt{1+\frac{\eta^{2}}{3}} \stackrel{57 \mathbf{F e}}{\Longrightarrow} \quad \Delta E_{q}=\frac{e Q \phi_{z z}}{2} \cdot \sqrt{1+\frac{\eta^{2}}{3}}
$$

Die Bedingungen für die Entstehung eines EFG sind [96]: 

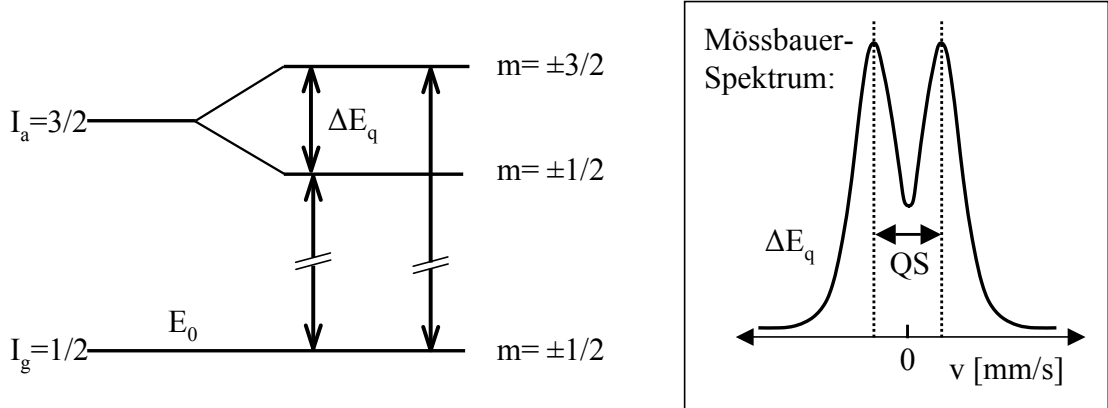

Abbildung 3.2: Elektische Quadrupol-Wechselwirkung und Quadrupolaufspaltung QS

1. Die Ladungsverteilung der Elektronen ist asymmetrisch, was verursacht werden kann durch a) Polarisation von gepaarten Elektronen in gefüllten Elektronenschalen oder b) durch nicht-sphärische, aber symmetrische Verteilung von Elektronen in teilgefüllten Orbitalen, wie z.B. die $3 d^{6}$-Konfiguration in $\mathrm{Fe}(\mathrm{II})$.

2. Äußere Einwirkung geladener Teilchen (Ionen oder Liganden). Besonders in Ionen-Kristallen ist dieser Effekt ausgeprägt.

Die Intensitäten beider Linien des Quadrupol-Dubletts können unterschiedlich sein, da sie nämlich abhängig vom Winkel $\theta$ zwischen dem EFG und der $\gamma$-Ausbreitungsrichtung [96] sind.

$$
\frac{m_{I}= \pm 3 / 2 \rightarrow \pm 1 / 2}{m_{I}= \pm 1 / 2 \rightarrow \pm 1 / 2}=\frac{1+\cos ^{2} \theta}{5 / 3-\cos ^{2} \theta}
$$

\subsubsection{Magnetische Dipol-Wechselwirkung und magnetische Aufspaltung}

In Anwesenheit eines Magnetfeldes $B$ am Kernort wird die Entartung der nuklearen Zustände durch die magnetische Wechselwirkungsenergie $E_{m}=-\boldsymbol{\mu} \cdot \mathbf{B}$ aufgehoben. Ein Zustand mit Spin $I$ spaltet in $(2 I+1)$ verschiedene Niveaus mit der Energie

$$
\Delta E_{m}=-g_{n} \mu_{n} m_{I} B
$$

auf. In Tab. 3.2 sind die Übergänge der einzelnen Niveaus gesondert aufgeführt. $\theta$ bezeichnet den Winkel

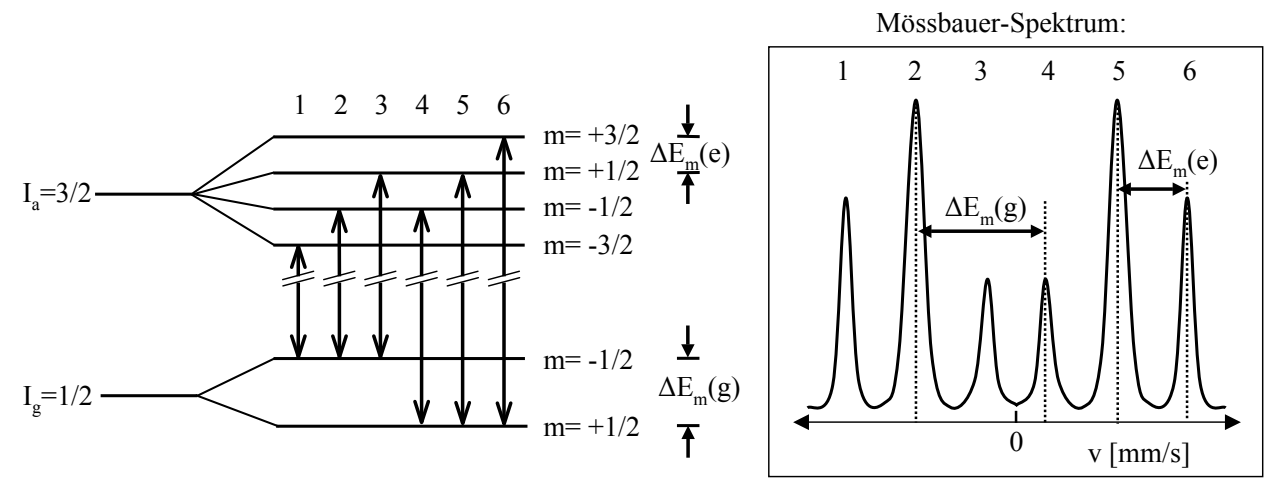

Abbildung 3.3: Magnetische Dipol-Wechselwirkung und magnetische Aufspaltung

zwischen Spin-Ausrichtung (magnetische Momente) und Richtung der $\gamma$-Aussendung. Für die Intensitätsverhältnisse der 6 Linien ergibt sich 3:x:1:1:x:3, wobei

$$
x=\frac{4 \sin ^{2} \theta}{1+\cos ^{2} \theta} .
$$

Für eine Magnetisierung in der Schichtebene $\left(\theta=90^{\circ}\right)$ erhält man ein Verhältnis von 3:4:1:1:4:3, wie es typischerweise bei CEM-Spektren zu erwarten ist. Liegt eine statistische Magnetisierungsverteilung im 
Tabelle 3.2: Winkelabhängigkeit der Mössbauer-Übergänge 96 .

\begin{tabular}{|c|c|c|}
\hline Übergang & $\Delta m$ & Winkelabhängigkeit \\
\hline \hline$+3 / 2 \rightarrow+1 / 2$ & -1 & $9 / 4 \cdot\left(1+\cos ^{2} \theta\right)$ \\
$-3 / 2 \rightarrow-1 / 2$ & +1 & $3 \sin ^{2} \theta$ \\
\hline$+1 / 2 \rightarrow+1 / 2$ & 0 & \\
$-1 / 2 \rightarrow-1 / 2$ & 0 & $3 / 4 \cdot\left(1+\cos ^{2} \theta\right)$ \\
\hline$-1 / 2 \rightarrow+1 / 2$ & +1 & \\
$+1 / 2 \rightarrow-1 / 2$ & -1 & \\
\hline
\end{tabular}

Raum vor $\left(\theta=54,7^{\circ}\right)$, so ist das Verhältnis 3:2:1:1:2:3. Tief im Inneren einer Probe (Bulk) tritt diese Situation auf und ist daher meist mit CXMS zu beobachten.

Es kann passieren, dass magnetische Dipol- und elektrische Quadrupol-Aufspaltung nebeneinander auftreten und sich beide Wechselwirkungen überlagern. Ist eine Wechselwirkung im Vergleich zur anderen sehr klein, so kann man sie in der quantenmechanischen Rechnung als Störungsterm behandeln. Ist z.B. die elektrische Quadrupol-Aufspaltung sehr klein, so erhält man für die Energie-Verschiebungen des ZeemanSextetts folgendes Bild (Abb. 3.4):
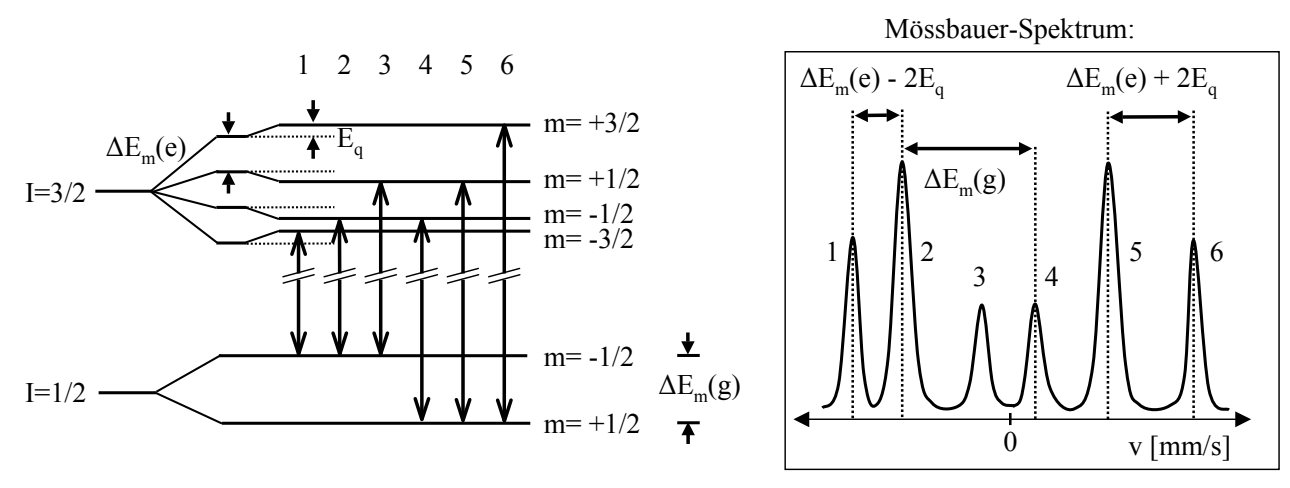

Abbildung 3.4: Gemischte Hyperfeinaufspaltung: Magnetische Dipol-Aufspaltung plus elektrische QuadrupolAufspaltung als Störungsterm

\subsubsection{Innere Konversion und Kernabregung}

In Abb. 3.5 ist das Abregungsschema des ${ }^{57}$ Fe gezeigt. Der angeregte Kern kann seine Energie in Form von

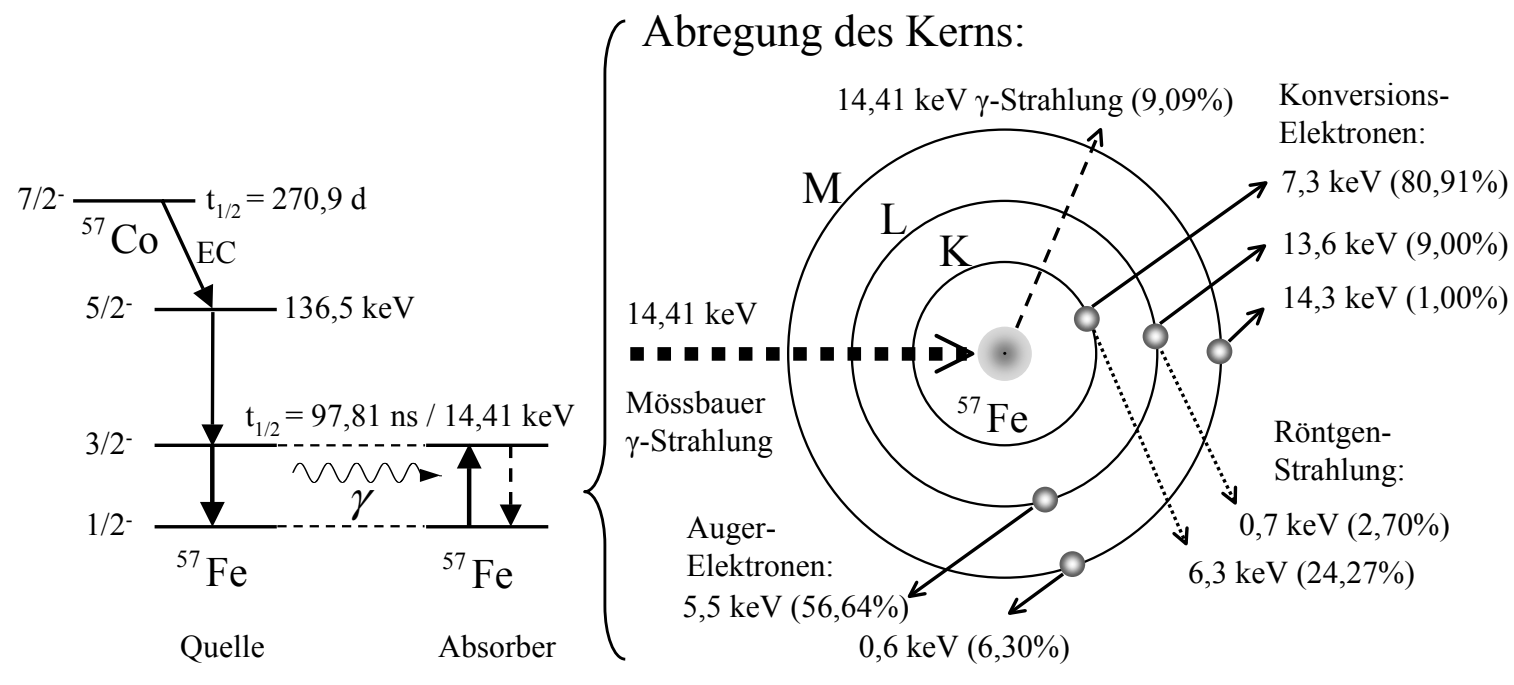

Abbildung 3.5: Abregungs-Schema von ${ }^{57} \mathrm{Fe}$ (nach [97, Werte nach [98 und Tab. 3.1]. 
$\gamma$-Strahlung oder über den konkurrierenden Prozess der inneren Konversion abgegeben. Bei diesem Mechanismus überlappen die Kernwellenfunktion und die Wellenfunktion der Elektronenhülle. Im Endeffekt wird dabei ein Elektron emittiert.

\subsubsection{Konversions-Elektronen- und Röntgenstrahlen-Mössbauerspektroskopie}

Für die Aufnahme der Konversions-Elektronen- und Konversions-Röntgenstrahlungs-Spektren (CEMS \& CXMS) wird bei Raumtemperatur ein Simultaneous Triple-Radiation Mössbauer Spectrometer (STRMS) benutzt [99]. Geometrie und Detektoranordnung sind in Abb. 3.6 skizziert: Die Quelle besteht aus ${ }^{57} \mathrm{Fe}$,

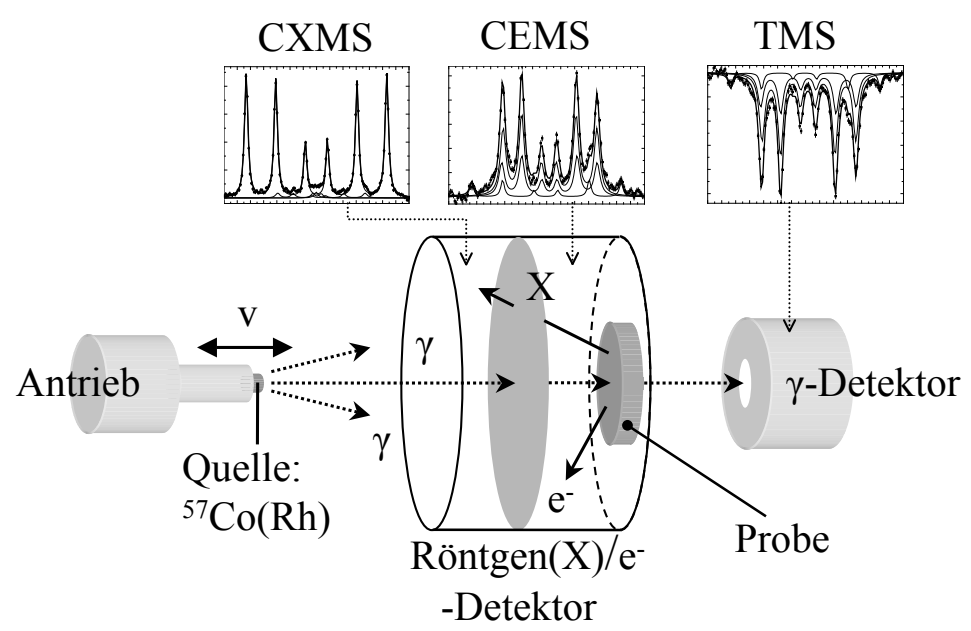

Abbildung 3.6: Aufbau des Transmissions(TMS)- und Konversions(CEMS/CXMS)-Mössbauer-Spektroskopie-Messplatzes

das von einer Rh-Matrix umgeben ist. Die Aktivität beträgt maximal $\approx 1 \mathrm{GBq}$. Die Quelle wird im constant-acceleration-Modus betrieben und bewegt sich mit Frequenzen um die $10 \mathrm{~Hz}$ auf die Probe zu und wieder weg. Die Geschwindigkeit $v$ der Quelle sorgt durch den Doppler-Effekt für die Energievariaqtion der Resonanzverstimmung

$$
\Delta E=E_{0}\left(1+\frac{v}{c}\right),
$$

wobei $v$ im Bereich einiger $\mathrm{mm} / \mathrm{s}$ liegt.

In TMS-Geometrie tritt die $\gamma$-Strahlung durch die Probe hindurch und wird in einem $\gamma$-Detektor registriert. Die Dicke der gemessenen Proben beträgt jedoch mindestens $1 \mathrm{~mm}$, so dass TMS hier nicht in Frage kommt. In einem anderen Verfahren wird in Rückstreugeometrie gemessen. Der angeregte Kern gibt dabei die Energie entweder als Konversions-Elektronen (CE) oder als Konversions-Röntgenstrahlung (CX) ab. Die Technik heißt daher Konversions-Elektronen- (CEMS) oder Konversions-RöntgenstrahlungMössbauer-Spektroskopie (CXMS). Die Elektronen werden in einem mit $\mathrm{He} / \mathrm{CH}_{4}$ und die Röntgenquanten in einem mit $\mathrm{Ar} / \mathrm{CH}_{4}$ durchströmten Proportional-Zähler detektiert [100] (s. Abb. 3.6]. Die Rohdaten werden in einem 1024-Kanal-Analysator aufgezeichnet [101] und die generierten Spektren anschließend standardmäßig durch Superponierung mehrerer Lorentz-Profile angepasst [102]. Die Fit-Parameter sind Isomerieverschiebung $\delta[\mathrm{mm} / \mathrm{s}]$, Quadrupolaufspaltung $\varepsilon[\mathrm{mm} / \mathrm{s}]$, FWHM-Profilbreite $\Gamma[\mathrm{mm} / \mathrm{s}]$, das magnetische Hyperfeinfeld $B[\mathrm{~T}]$ und die relativen Profil-Intensitäten.

Die Geschwindigkeitseichung erfolgt bei Raumtemperatur mit einer $25 \mu \mathrm{m}$ dicken $\alpha$-Fe-Folie. Weil CEMS eine Informationstiefe von ca. $150 \mathrm{~nm}$ aufweist, ist sie sehr gut geeignet, um die obersten Oberflächenschichten zu untersuchen. Demgegenüber reicht CXMS bis in Tiefen von 10-20 $\mu \mathrm{m}$ 103, 104 .

\subsection{Röntgen-Diffraktometrie (XRD) und Kristallographie}

Die Röntgen-Diffraktometrie [89, 105, 90, 106, 107, 108] liefert Informationen über die atomare Struktur und interatomare Abstände, die in der Größenordnung $10^{-10} \mathrm{~m}=1 \AA$ liegen. Die Wellenlänge der elektro 
magnetischen Strahlung muss demzufolge in derselben Größenordung liegen, d.h. $h f=h c / \lambda \simeq 1,2 \cdot 10^{4}$ eV. Diese Energien liegen im Bereich der Röntgenstrahlung.

Das Kristallgitter wird beschrieben durch ein System periodischer, dreidimensionaler Anordnungen. Mathematisch gesehen ist ein Gitter die Faltung eines Punktgitters mit einer Basis(einheit): Die Basis wird in regelmäßigen Abständen aneinandergereiht, und das Punktgitter wird derart gewählt, dass jeder Gitterpunkt zu einer bestimmten Atom- oder Molekülgruppe gehört. Diese kleinsten räumlichen Einheiten heißen Elementarzellen, die genau einem Gitterpunkt und einer Basis zugeordnet sind; die Elementarzellen werden durch 3 linear unabhängige Grundvektoren $\mathbf{a}_{1}, \mathbf{a}_{2}, \mathbf{a}_{3}$ aufgespannt. Die Elementarzellen bauen das gesamte Kristallgitter durch Symmetrieoperationen auf. Dadurch ergeben sich 7 Kristallsysteme und 14 elementare Bravais-Gitter (Tab. 3.3. In Abb. 3.7 ist ein Beispiel für ein kubisch-flächenzentriertes Gitter

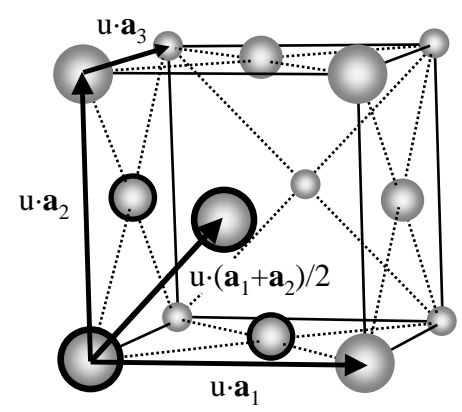

Abbildung 3.7: Kubisch-flächenzentriertes Bravaisgitter (Gitterparameter $u$ ). Die Basis bilden hier 4 Atome (schwarz umrundet dargestellt).

(fcc) gegeben. Dabei bedeutet, dass die Elementarzelle bei $P$ nur an den Ecken besetzt (primitiv), $C$ : in Tabelle 3.3: Gitterkonstanten- und Winkelbeziehungen in Bravais-Gittern.

\begin{tabular}{|c||c|c|c|}
\hline Raumgitter & Winkel & $\begin{array}{c}\text { Gitter- } \\
\text { konstanten }\end{array}$ & $\begin{array}{c}\text { Zentrierungs- } \\
\text { typen }\end{array}$ \\
\hline \hline triklin & $\alpha_{1}, \alpha_{2}, \alpha_{3} \neq 90^{\circ}$ & $a_{1} \neq a_{2} \neq a_{3}$ & $P$ \\
\hline monoklin & $\alpha_{1}=\alpha_{2}=90^{\circ}, \alpha_{3} \neq 90^{\circ}$ & $a_{1} \neq a_{2} \neq a_{3}$ & $P, C$ \\
\hline orthorhombisch & $\alpha_{1}=\alpha_{2}=\alpha_{3}=90^{\circ}$ & $a_{1} \neq a_{2} \neq a_{3}$ & $P, C, I, F$ \\
\hline hexagonal & $\alpha_{1}=60^{\circ}, \alpha_{2}=\alpha_{3}=90^{\circ}$ & $a_{1} \neq a_{2}=a_{3}$ & $P$ \\
\hline trigonal & $\alpha_{1}=\alpha_{2}=\alpha_{3} \neq 90^{\circ}$ & $a_{1}=a_{2}=a_{3}$ & $P$ \\
\hline tetragonal & $\alpha_{1}=\alpha_{2}=\alpha_{3}=90^{\circ}$ & $a_{1}=a_{2} \neq a_{3}$ & $P, I$ \\
\hline kubisch & $\alpha_{1}=\alpha_{2}=\alpha_{3}=90^{\circ}$ & $a_{1}=a_{2}=a_{3}$ & $P, I, F$ \\
\hline
\end{tabular}

den Basisflächen zentriert, $I$ : innenzentriert und $F$ : flächenzentriert ist.

\subsubsection{Beugungsbedingung}

Ein Vektor des Gitters kann als

$$
\mathbf{r}=\sum_{i} n_{i} \cdot \mathbf{a}_{i} \quad, \quad n_{i} \in \mathbb{N}
$$

dargestellt werden. Ein kubisches Gitter sei durch die 3 Translations-(Grund-)vektoren $\mathbf{a}_{1}, \mathbf{a}_{2}, \mathbf{a}_{3}$ charakterisiert, das ein rechtwinkliges Koordinatensystem beschreibt. Legt man irgendeine Ebene, die beispielsweise Reflexionsebene ist, willkürlich in das Kristallgitter, so schneidet diese das Koordinatensystem bei ganzzahligen Vielfachen der Translationsvektoren $a \mathbf{a}_{1}, b \mathbf{a}_{2}, c \mathbf{a}_{3}$. Die Kehrwerte der Achsenabschnitte $a, b, c$ werden mit dem kleinsten Vielfachen multipliziert, das zu einer ganzen Zahl führt. Diese Zahlen $h, k, l$ heißen Millersche Indizes (M.I.). Gitterebenen, die beispielsweise parallel (Schnittpunkt befindet sich im Unendlichen) zu Würfelflächen liegen, sind somit (h00), (0k0) oder (001)-Ebenen. Negative M.I. werden als $(\bar{h} \bar{k} \bar{l})$ geschrieben. In Frage kommende Ebenen werden nicht durch den Nullpunkt gelegt, 
sondern sie werden gegebenenfalls mindestens um eine entsprechende Grundvektorlänge verschoben. Die Kristallrichtungen werden mit $[h k l]$ indiziert.

\section{Reziprokes Gitter}

Beugungserscheinungen und andere strukturanalytische Probleme können auf elegante Weise mit dem Konzept des reziproken Gitters behandelt werden. Mathematisch handelt es sich um eine Fourier-Transformation des realen Kristallgitters. Die Grundvektoren des reziproken Gitters $\mathbf{a}_{i}^{*}$ und die des realen Gitters $\mathbf{a}_{j}$ sind durch folgende Gleichungen verknüpft:

$$
\mathbf{a}_{i}^{*} \cdot \mathbf{a}_{j}=2 \pi \delta_{i j}\left\{\begin{array}{ccc}
0 & \text { für } & i \neq j \\
2 \pi & \text { für } & i=j
\end{array} \quad \text { mit } i, j=1,2,3\right.
$$

Das bedeutet

$$
\mathbf{a}_{i}^{*} \perp \mathbf{a}_{j} \vee \mathbf{a}_{i}^{*} \perp \mathbf{a}_{k} \quad \Rightarrow \quad \mathbf{a}_{i}^{*} \|\left(\mathbf{a}_{j} \times \mathbf{a}_{k}\right) \quad \text { mit } i, j, k=1,2,3 \text { (paarweise verschieden) }
$$

Die Gleichung

$$
\mathbf{a}_{i}^{*}=2 \pi \cdot \frac{\mathbf{a}_{j} \times \mathbf{a}_{k}}{\mathbf{a}_{i} \cdot \mathbf{a}_{j} \times \mathbf{a}_{k}}
$$

genügt den Gl. (3.32) und 3.33). Der Ausdruck $\mathbf{a}_{i} \cdot \mathbf{a}_{j} \times \mathbf{a}_{k}$ ist dabei das Volumen der Einheitszelle. Ein beliebiger Vektor $\mathbf{R}^{*}$ des reziproken Gitters ist durch

$$
\mathbf{R}^{*}=\sum_{i} m_{i} \cdot \mathbf{a}_{i}^{*} \quad, \quad m_{i} \in \mathbb{N}
$$

darstellbar. Das Produkt

$$
\mathbf{r} \cdot \mathbf{R}^{*}=\left(\sum_{i} n_{i} \cdot \mathbf{a}_{i}\right) \cdot\left(\sum_{i} m_{i} \cdot \mathbf{a}_{i}^{*}\right)=2 \pi\left(\sum_{i} n_{i} \cdot m_{i}\right)=2 \pi \cdot c \quad, \quad c \in \mathbb{N}
$$

ist eine ganze Zahl. Wird diese Bedingung erfüllt, so erfolgt konstruktive Interferenz.

Die Beugung kann auf 2 äquivalenten Wegen beschrieben werden, die in Abb. 3.8 skizziert sind: Die Bragg'sche Bedingung geht von parallelen Scharen von reflektierenden Netzebenen aus, die im Abstand $d$ voneinander angeordnet sind, während die v. Laue'sche Bedingung regelmäßig angeordnete, isotrope Streuzentren voraussetzt. Um konstruktive Interferenz zu erreichen, müssen die Weglängenunterschiede

a) Bragg:
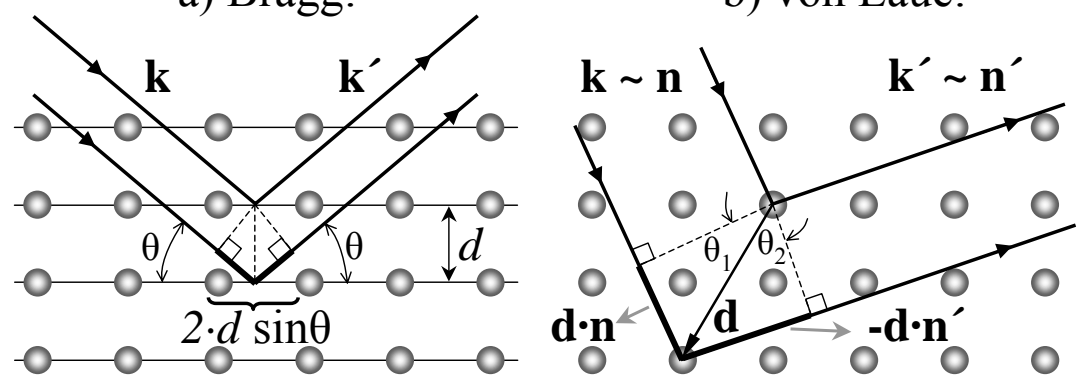

Abbildung 3.8: a) Bragg'sche und b) v. Laue'sche Reflexionsbedingung.

einfach ein ganzzahliges Vielfaches der Wellenlänge $\lambda$ sein, woraus sich die Bragg-Gleichung

$$
2 d \cdot \sin \phi=n \cdot \lambda \quad(n \in \mathbb{N})
$$

ergibt (Abb. 3.8a). Im Fall der v. Laue'schen Reflexionsbedingung (Abb. 3.8b) betrachtet man 2 beliebige Streuzentren, die sich im Abstand $d=|\mathbf{d}|$ befinden. Die Röntgenstrahlung mit der Wellenlänge $\lambda$ fällt aus einer $\mathbf{n}$-Richtung ein, deren Wellenvektor $(\mathbf{k})$ durch den Einheitsvektor $(\mathbf{n})$ und die Wellenlänge $(\lambda)$ wie folgt gegeben ist:

$$
\mathbf{k}=\frac{2 \pi \mathbf{n}}{\lambda}
$$


Entsprechendes gilt für die ausgehende, gestreute Welle.

$$
\mathbf{k}^{\prime}=\frac{2 \pi \mathbf{n}^{\prime}}{\lambda}
$$

Die Bedingung für Interferenz ergibt sich analog der Bragg'schen Bedingung aus den Wegunterschieden:

$$
d \cdot \cos \theta_{1}+d \cdot \cos \theta_{2}=\mathbf{d} \cdot \mathbf{n}-\mathbf{d} \cdot \mathbf{n}^{\prime}=\mathbf{d} \cdot\left(\mathbf{n}-\mathbf{n}^{\prime}\right) \stackrel{!}{=} n \lambda
$$

Für den Wellenvektor gilt somit

$$
\mathbf{d} \cdot\left(\mathbf{k}-\mathbf{k}^{\prime}\right)=2 \pi m
$$

was gleichbedeutend mit der Identität

$$
\exp \left(i \mathbf{d} \cdot\left(\mathbf{k}-\mathbf{k}^{\prime}\right)\right)=1
$$

ist. Damit alle zur konstruktiven Interferenz beitragenden Streuzentren berücksichtigt werden, ersetzt man den Verschiebungsvektor $\mathbf{d}$ zweier Zentren durch den Gittervektor $\mathbf{r}$ des Bravaisgitters, weil die Gitterplätze relativ zueinander um $\mathbf{r}$ verschoben sind und somit alle $\mathbf{d}$ in den Vektoren $\mathbf{r}$ enthalten sind. Aufgrund des Streuprozesses ändert sich der Wellenvektor um

$$
\mathbf{k}-\mathbf{k}^{\prime}=\Delta \mathbf{k}=\mathbf{K}
$$

den sog. Streuvektor. Die Interferenz-Bedingung lautet somit:

$$
\begin{gathered}
\exp (i \mathbf{r} \cdot \mathbf{K})=1 \quad(\Leftrightarrow \quad \mathbf{r} \cdot \mathbf{K}=2 \pi n) \\
\Delta \mathbf{k} \cdot \mathbf{r}=\Delta \mathbf{k} \cdot\left(\sum_{i} n_{i} \cdot \mathbf{a}_{i}\right)=2 \pi c \quad, \quad c \in \mathbb{N}
\end{gathered}
$$

Bei elastischer Streuung ist der Betrag des ein- und auslaufenden Wellenvektors gleich, d.h. $|\mathbf{k}|=\left|\mathbf{k}^{\prime}\right|$, im Teilchenbild werden die Photonen also elastisch am Kristallgitter gestreut $\hbar \mathbf{k}=\hbar \mathbf{k}^{\prime}$. Geometisch veranschaulicht bedeutet Gl. 3.43. Wenn beide Wellenvektoren $\mathbf{k}$ und $\mathbf{k}^{\prime}$ von einem Punkt im reziproken Gitter ausgehen, dann enden deren Endpunkte auf einem Kreis (oder Kugel).

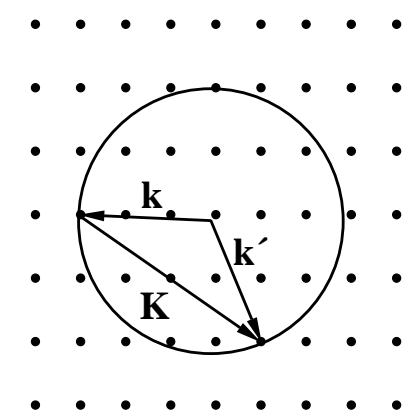

Abbildung 3.9: Ewald-Konstruktion zur Streuung zweier Photonen im reziproken Gitter.

Die Abb. 3.9 stellt diese Ewald-Kugel dar. Nur wenn diese Bedingung erfüllt ist, kommt ein Beugungsreflex zustande. Die Ebenenabstände $d$, die Millerschen Indizes und die Gitterparameter $a, b, c$ hängen für das kubische Gitter wie folgt voneinander ab [109]:

$$
\frac{1}{d^{2}}=\frac{h^{2}+k^{2}+l^{2}}{a^{2}}
$$

mit der Braggbedingung (Gl. 3.37, $n=1$ ) ergibt das für den Gitterparameter $a$ :

$$
a=\frac{\lambda}{2 \sin \theta} \sqrt{\left(h^{2}+k^{2}+l^{2}\right)}
$$


In der bisherigen Behandlung der Röntgenbeugung sind nur die Bedingungen aufgeführt, unter denen Reflexe in bestimmten Richtungen auftreten. Desweiteren können noch Aussagen über die entsprechende Intensität getroffen werden. Die Intensität der reflektierten Strahlen hängt im Kristallgitter von den BasisAtomen der Einheitszelle, deren individuellen Streueigenschaften sowie der Temperatur des Kristallgitters ab.

Der Phasenunterschied zweier Röntgenstrahlen beträgt gemäß Abb. (3.8b) und der Gl. (3.40).

$$
\phi(\mathbf{d})=\frac{2 \pi}{\lambda} \mathbf{d} \cdot\left(\mathbf{n}-\mathbf{n}^{\prime}\right)=\mathbf{K} \cdot \mathbf{d}
$$

Die Amplitude der elektrische Feldstärke ist gegeben durch [90,105

$$
F=\int_{\text {Zelle }} n(\mathbf{d}) \cdot \exp (i \phi(\mathbf{d})) d V=\int_{\text {Zelle }} n(\mathbf{d}) \cdot \exp (\mathbf{K} \cdot \mathbf{d}) d V
$$

$n(\mathbf{d})$ ist die Elektronendichte in der Einheitszelle. Der Amplitudenfaktor $f$ ist die Fourier-Transformierte der Elektronendichte. Für den atomaren Strukturfaktor $F$ der Elementarzelle gilt:

$$
F_{h k l}=\sum_{i} f_{i} \exp \left[2 \pi i\left(\frac{h \cdot x}{a}+\frac{k \cdot y}{b}+\frac{l \cdot z}{c}\right)\right]
$$

$a, b$ und $c$ sind die Dimensionen der Einheitszelle, $x, y$ und $z$ bezeichnen die Koordinaten des $j$-ten Atoms. Die Reflexe werden somit nicht nur durch die Atompositionen, sondern auch durch die Atomspezies bestimmt.

\subsubsection{Lorentz-Polarisations-Faktor}

Die gestreute Intensität ist stark abhängig vom Winkel $\theta$ zwischen der Einfalls- und Streu-Richtung der Röntgenstrahlung. Diese winkelabhängige Intensitätsverteilung wird durch den Lorentz-PolarisationsFaktor erfasst, der sich sich aus 2 Anteilen zusammensetzt:

Der Lorentz-Faktor $L$ erfasst die Geometrie und Orientierung der reflektierenden Ebenen [108]:

$$
L=\frac{1}{\sin ^{2} \theta \cdot \cos \theta}
$$

Der Polarisations-Faktor rührt von der Röntgen-Streuung an einem Elektron her: Von einer Röntgenquelle emittierte Strahlung ist unpolarisiert, jedoch enthält der gestreute Strahl einen polarisierten Anteil. Die

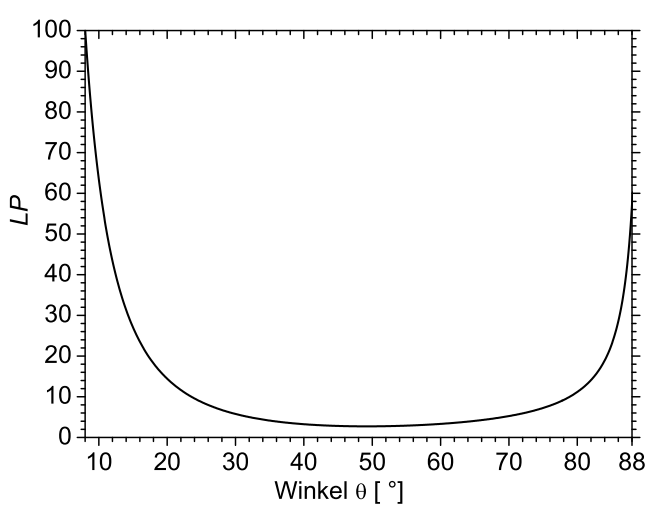

Abbildung 3.10: Lorentz-Polarisations-Faktor: Winkel-Abhängigkeit der Intensität.

Intensitätsverteilung ist winkelabhängig und nach der Thomson-Gleichung [110]:

$$
I \sim I_{0} \cdot \frac{1+\cos ^{2} 2 \theta}{2}
$$


Beide Terme ergeben zusammen den Lorentz-Polarisations-Faktor

$$
L P=\frac{1+\cos ^{2}(2 \theta)}{\sin ^{2} \theta \cdot \cos \theta}
$$

Die Winkelabhängigkeit ist in Abb. (3.10) von $8^{\circ}-88^{\circ}$ dargestellt. Für die Gesamtintensität des gestreuten Strahls ergibt sich schließlich:

$$
I_{h k l}=\left|F_{h k l}\right|^{2} \cdot M\left(\frac{1+\cos ^{2} 2 \theta}{\sin ^{2} \theta \cos \theta}\right) \cdot \exp \left[-2 f_{D}(T)\right],
$$

wobei $M$ die Multiplizität ist. Diese gibt an wieviel äquivalente Ebenen $\{h k l\}$ zur Bragg-Streuung beitragen. In einem kubischen Gitter sind beispielsweise die Ebenen $( \pm h, 00),(0, \pm k, 0)$ und $(00, \pm l)$ gleichwertig und erfüllen bei festem $\theta$ die Gl. (3.47).

\subsubsection{Eindringtiefe und Geometrie}

Die Röntgenmessungen mit einem BRUKER ${ }^{\circledR}$ AXS Diffractometer durchgeführt. Das Gerät verwendet $\mathrm{Cu}-K_{\alpha}$ Strahlung der Wellenlänge $\lambda=1,54 \AA$ und einen LiF-Einkristall-Monochromator. Die Messungen wurden in grazing incidence-Geometrie durchgeführt, die in Abb. 3.11 skizziert ist. Der Röntgenstrahl fällt in festem Winkel $\alpha$ auf die Probe. Der Winkel zwischen dem einfallenden und dem austretenden wird mit $2 \theta$ bezeichnet und beträgt bei allen Messungen $35^{\circ} \leq 2 \theta \leq 100^{\circ}$. Die Eindringtiefen für verschiedene

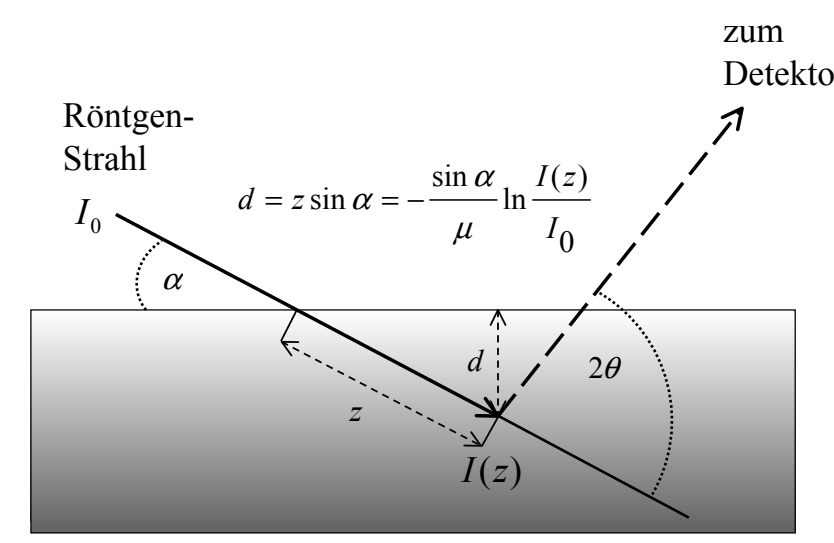

Abbildung 3.11: Intensitätsabfall bei GIXRD-Geometrie. Nach einer Strecke $z$, die der Tiefe $d=z \cdot \sin \alpha$ entspricht, ist die Intensität der Röntgenstrahlung $I_{0}$ auf $I(z)$ abgefallen.

Einfallswinkel sind in Tab. (3.4) aufgeführt: Die Tiefen wurden unter der Annahme errechnet, dass 90\% der

Tabelle 3.4: Eindringtiefe $[\mu \mathrm{m}]$ der Röntgenstrahlung in $\mathrm{Fe}, \mathrm{Fe}_{3} \mathrm{C}$, Edelstahl (SS) und $\mathrm{C}$ bei $90 \%$ Absorption unter verschiedenen Einfallswinkeln. Die mittlere Dichte des Kohlenstoffs ist $\rho=2,0 \mathrm{~g} / \mathrm{cm}^{3}$.

\begin{tabular}{|c||c|c|c|c|c|}
\hline \multicolumn{1}{|c||}{ Material } & \multicolumn{5}{c|}{ Einfallswinkel [ ${ }^{\circ}$ ] } \\
& 0,7 & 1 & 3 & 5 & 10 \\
\hline \hline $\mathrm{Fe}$ & 0,11 & 0,15 & 0,45 & 0,72 & 1,26 \\
\hline $\mathrm{Fe}_{3} \mathrm{C}$ & 0,15 & 0,21 & 0,62 & 0,99 & 1,7 \\
\hline $\mathrm{SS}$ & - & 0,17 & 0,51 & 0,81 & 1,4 \\
\hline $\mathrm{C}$ & 33,2 & 47 & 135 & 214 & 378 \\
\hline
\end{tabular}

einfallenden Röntgenstrahlung, d.h. $I(z) / I_{0}=0,1$, absorbiert werden. Das Programm ist Bestandteil des Röntgengerätes. Der Einfallswinkel von $1^{\circ}$ liefert eine Informationstiefe von ca. $150 \mathrm{~nm}$ und ist mit der der CEM-Spektroskopie ungefähr gleich. Somit können Resultate beider Messungen miteinander verglichen werden. 


\subsection{Extended X-Ray Absorption Fine-Structure (EXAFS)}

Tritt Röntgenstrahlung durch Materie hindurch, so wird diese durch 3 Absorptionsmechanismen gedämpft, die jeweils mit unterschiedlicher energieabhäniger Wahrscheinlichkeit auftreten; dazu gehören 1. der Photoeffekt, 2. der Comptoneffekt und 3. die Paarbildung. Die Gesamtdämpfung wird beschrieben durch die Dämpfungsfunktion

$$
I(z)=I_{0} \cdot \exp (-\mu \cdot z)
$$

$z$ ist die Dicke des durchstrahlten Materials und $\mu$ dessen Absorptionskoeffizient. Der Absorptionskoeffiezient setzt sich wegen gegenseitiger Unabhängigkeit aus den Absorptionskoeffizienten der Einzelprozesse additiv zusammen:

$$
\mu=\mu_{\text {Photo }}+\mu_{\text {Compton }}+\mu_{\text {Paar }}
$$

Der in obiger Formel als konstant angenommene Absorptionskoeffizient $\mu$ hängt tatsächlich selbst von der Energie und der Kernladungszahl $Z$ ab.

Reicht die Photonenenergie $E_{P}$ aus, um einen Photoeffekt in der $K$-Schale auszulösen, so steigt der Absorptionskoeffizient sprunghaft an (Absorptionskante) und nimmt anschließend ungefähr mit $E^{-3}$ wieder ab. Die kinetische Energie $E$ des Photoelektrons ist

$$
E=\hbar \omega-E_{\text {bind }}
$$

$E_{\text {bind }}$ ist die Bindungsenergie des emittierten Elektrons. Über die Beziehung $E=\mathrm{p}^{2} / 2 m=(\mathbf{k} \hbar)^{2} / 2 m$ ist der Wellenvektor des emittierten Elektrons

$$
k=|\mathbf{k}|=\sqrt{\frac{2 m}{\hbar^{2}}\left(\hbar \omega-E_{\text {bind }}\right)}
$$

Die emittierten Elektronen, die als auslaufende Kugelwellen an den starr angeordneten Nachbaratomen gestreut werden, superponieren und erzeugen aufgrund ihrer unterschiedlichen Phasenlage zueinander ein kompliziertes Wellenfeld. Der höherenergetische Teil der Absorptionskante weist demzufolge eine charakteristische Oszillation $\mu$ auf, die sog. x-ray absorption fine structure (XAFS). Diese Modulation ist dem linearen Verlauf der Absorption $\mu_{0}$, die dem freien Atom entsprechen würde, überlagert und man definiert das normierte XAFS-Spektrum durch

$$
\chi(E)=\frac{\mu_{0}(E)-\mu(E)}{\mu_{0}(E)}
$$

XAFS ist abhängig von der Anzahl und Typ der Atome in der Koordinations-Schale des streuenden Atoms. Für Eisen beträgt die Ionisationsenergie für ein Elektron der $K$-Schale $7112 \mathrm{eV}$ [111]. Die XAFSRegion kann zudem in 2 Hauptbereiche unterteilt werden [110 112] (Abb. (3.12): Im Bereich von der Absorptionskante bis ca. $30 \mathrm{eV}$ tritt XANES (x-ray absorption near edge structure) auf, die im wesentlichen durch Mehrfach-Streuung bestimmt wird. Der schwächer oszillierende Bereich jenseits XANES bis ca. 1000 $\mathrm{eV}$ ist die Extended XAFS (EXAFS), die strukturelle Informationen über die atomare Umgebung enthält. Die Umrechnung von der Energie- in die $k$-Skala erfolgt mit Gl. (3.58). Gewöhnlich ist $\chi$ als Funktion von $k$ definiert. Gemäß der short-range-order Streutheorie [113 114 115 116] ergibt sich $\chi(k)$ in der Form [117]:

$$
\chi(k)=\frac{1}{k} \sum_{\substack{\text { Koordinations- } \\ \text { schale } i}} \frac{N_{i}}{r_{i}^{2}} S_{0}^{2}(k) F_{i}(k) \exp \left(\frac{-2 r_{i}}{\lambda(k)}\right) \exp \left(-2 \sigma_{i}^{2} k^{2}\right) \sin \left(2 k r_{i}+\phi_{i}(k)\right),
$$

die Summe erstreckt sich über die Koordinationsschalen des Zentralatoms; die strukturellen Parameter sind:

1. $N_{i}$ ist die Anzahl der Atome in der $i$-ten Koordinations-Schale (Koordinationszahl),

2. $r_{i}$ die interatomaren Abstände zwischen dem streuenden Zentral- und den Nachbaratomen,

3. $F_{i}(k)$ die Rückstreu-Amplitude, 


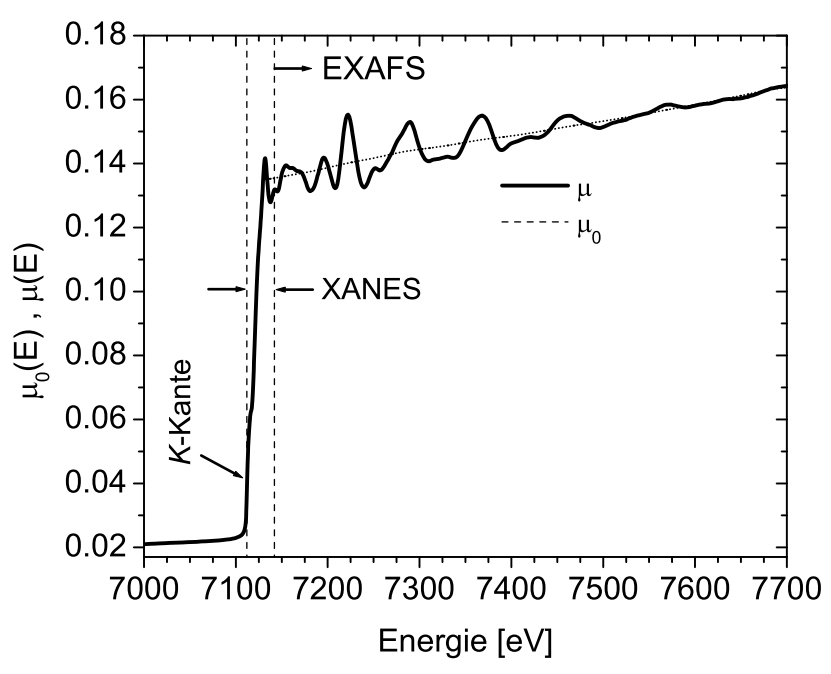

Abbildung 3.12: $K$-Schalen-Absorptionskoeffizient $\quad \mu(E)$ bzw. $\mu_{0}(E)$ von Eisen und die unterschiedlichen Regionen der XAFS. Die extrahierten $\chi(k)$-Daten sind in Abb. 3.13 dargestellt.

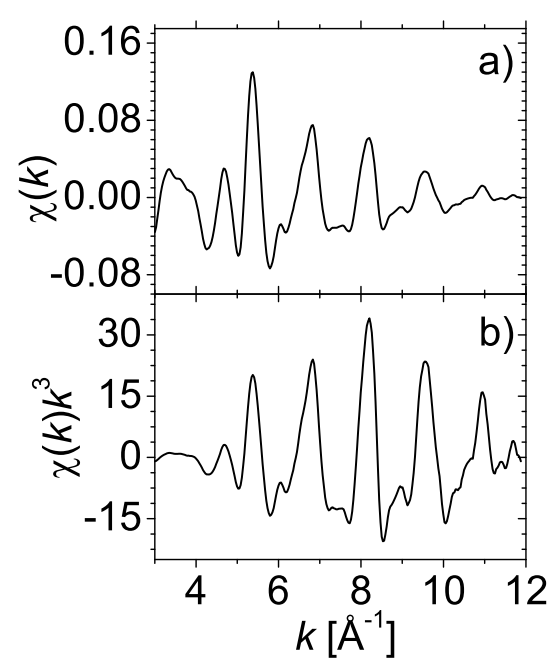

Abbildung 3.13: a) ungewichtete $\chi(k)$-Daten. b) der Gewichtungsfaktor $k^{3}$ betont höhere $k$-Werte und verteilt sie symmetrischer über das $k$ Intervall.

4. $S_{0}^{2}(k)$ beschreibt Vielkörper-Effekte, sog. shake-up- und shake-off-Prozesse, die durch elektronische Mehrfachanregungen des absorbierenden Atoms zustandekommen [112],

5. $\phi_{i}(k)$ ist die Phasenverschiebung, die das streuende Photoelektron aufgrund des Atom-Potenzials erfährt [118],

6. $\lambda(k)$ die freie Weglänge des Elektrons,

7. der Debye-Waller ähnliche Faktor $\sigma_{i}$ beschreibt die Schwankung der interatomaren Abstände (Bindungslänge); der Faktor $\sigma$ berücksichtigt sowohl thermische Vibration $\sigma_{\text {vib }}$ als auch statische, strukturell bedingte Unordnung $\sigma_{\text {stat. }}$. Es gilt $\sigma^{2}=\sigma_{\mathrm{vib}}^{2}+\sigma_{\text {stat }}^{2}[112$.

Der Debye-Waller-Faktor ist folgendermaßen gegeben

$$
\sigma_{i}=\sqrt{\left\langle\left[\mathbf{r}_{i} \cdot\left(\mathbf{u}_{i}-\mathbf{u}_{0}\right)\right]^{2}\right\rangle}
$$

Abb. (3.14) skizziert die Verschiebung zweier Atome gegeneinander: Atom $A$ absorbiert das Röntgenquant und liegt im Ursprung, $B_{i}$ ist ein Nachbaratom, das im Gleichgewicht um $\mathbf{r}_{i}$ relativ zu $A$ verschoben ist. Die Verrückungen aus ihrer Gleichgewichtslage sind $\mathbf{u}_{0}$ bzw. $\mathbf{u}_{i}$. Es ist anzumerken, dass sich der

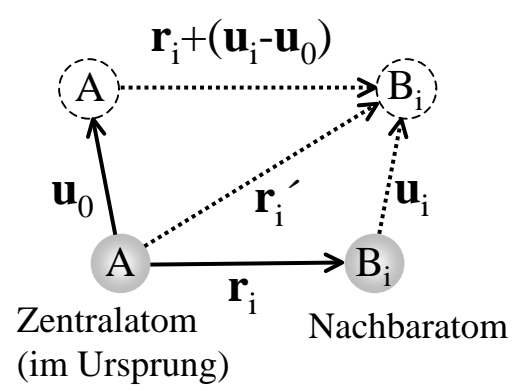

Abbildung 3.14: Gleichgewichts (-)- und instantane Positionen (..) und Verschiebungsvektoren zweier Atome (nach 112]).

EXAFS Debye-Waller-Faktor von dem der Röntgenstreuung bzw. Mössbauer-Effektes durch einen sog. Korrelations-Term (KT) unterscheidet [117, 112], denn Gl. (3.61) ergibt

$$
\sigma_{i}^{2}=\left\langle\left(\mathbf{r}_{i} \cdot \mathbf{u}_{i}\right)^{2}\right\rangle+\left\langle\left(\mathbf{r}_{i} \cdot \mathbf{u}_{0}\right)^{2}\right\rangle-\underbrace{2\left\langle\left(\mathbf{r}_{i} \cdot \mathbf{u}_{i}\right) \cdot\left(\mathbf{r}_{0} \cdot \mathbf{u}_{0}\right)\right\rangle}_{K T}
$$


Dieser Term ist besonders für die erste Koordinationsschale wegen der starken Bindung zwischen Zentralund Nachbaratome wichtig [112]. Bei der Auswertung muss man beachten, dass gemäß Gl. (3.60) die EXAFS aus den Beiträgen jeder Koordinations-Schale zusammensetzt sind. Um den Absorptionkoeffizienten des freien Atoms $\mu_{0}$ zu berechnen, fittet man ein Polynom oder ein kubischen Spline an die experimentellen Daten $\mu$ an (s. Abb. (3.12). Die Daten-Analyse erfolgte mit dem Programm VIPER 119 120. Um vom $k$-Raum in den $r$-Raum zu gelangen, muss man eine Fourier-Transformation durchführen [112]:

$$
\chi(k) \stackrel{\mathrm{FT}}{\Longrightarrow} \mathcal{F}\{\chi(k)\}=\tilde{\chi}(r)
$$

Da nur ein bestimmter Bereich der Fourier-Transformation unterzogen wird, müssen die Ränder des Intervalls möglichst glatt auf Null gehen, um im Spektrum verfälschende Peaks zu minimieren. Dazu werden die Daten $\chi(k)$ mit einer Fensterfunktion $\mathcal{W}(k)$ gefaltet, z.B. der hier benutzten Hanning-Funktion:

$$
\mathcal{W}(k)=\left\{\begin{array}{ccc}
\cos ^{2}\left(\frac{k-k_{0}}{\Delta k}\right) & \text { für } & \left|k-k_{0}\right|<\Delta k \\
0 & \text { für } & \left|k-k_{0}\right|>\Delta k
\end{array}\right.
$$

$\Delta k$ ist das gewählte Intervall $k_{\min }-k_{\max }$ und $k_{0}$ die Intervallmitte. Weil die Oszillationen für hohe $k$-Werte schnell abfallen, wird $\chi(k)$ mit dem Faktor $k^{n}, n=1,2,3$ gewichtet, um eine möglichst symmetrischen Verlauf von $\chi(k) k^{n}$ zu erzielen. Man erhält somit die Fourier-Transformierte als:

$$
\tilde{\chi}(k)=\int_{\min }^{\max } \mathcal{W}(k) k^{n} \exp (\mathrm{i} k r) \chi(k) d k
$$

Abb. 3.15 zeigt den experimentellen Aufbau: Die Elektronen werden vorbeschleunigt und in den eigentlichen Speicherring injiziert. Das Restgas im Ring hat zur Folge, dass die Elektronen kontinuierlich Energie verlieren und mit der Wand kollidieren. Im Laufe der Zeit nimmt daher die Intensität der Strahlung ab. Daher muss in bestimmten Intervallen (z.B. 12 Stunden) eine neue Injektion vorgenommen werden. Die Röntgenstrahlung erreicht über einen $\mathrm{Si}(111)$-Monochromator die Ionisationskammer. Die Differenz der Photoströme der Referenzkammer $I_{0}$ und der Probenkammer $I$ liefert die Oszillationen $\mu(E)$.

Die bemerkenswerte Eigenschaft der Synchrotron-Strahlung ist, dass sie neben ihrer linearen Polarisation [121] eine enorme Brillianz $B$ in der Größenordnung $10^{20}$ Photonen $/\left(\mathrm{mm}^{2} \cdot \mathrm{mrad}^{2} \cdot 0,1 \% \mathrm{BW}\right)$ aufweist [107] (BW = Bandbreite), wenn sie in Anlagen der 3. Generation erzeugt wird und liegt somit $10^{12}$-mal über dem Wert der herkömmlichen Labor-Geräte.

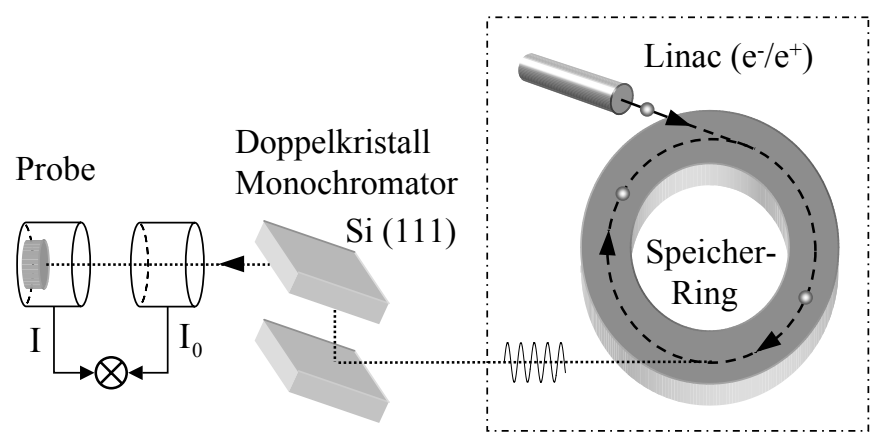

Abbildung 3.15: Prinzipieller Strahlengang und Aufbau eines Synchrotrons.

\subsection{Rutherford Backscattering Spectrometry (RBS)}

Bei der Rutherford Backscattering Spectromerty werden $\alpha$-Teilchen elastisch an den zu untersuchenden Target-Atomen zurückgestreut. Das Energiespektrum der $\alpha$-Patikel enthält Information über die Tiefenprofile der Elemente, die in der Probe enthalten sind. Das Prinzip ist in Abb. (3.16) dargestellt: Die RBS-Experimente wurden am IONAS-Beschleuniger in Göttingen durchgeführt [122]. Die He ${ }^{++}$-Teilchen der Masse $M_{\alpha}=4 \mathrm{amu}$ wurden mit einer Anfangsenergie von $900 \mathrm{keV}$ an den Targetatomen der Masse $M_{t}$ 


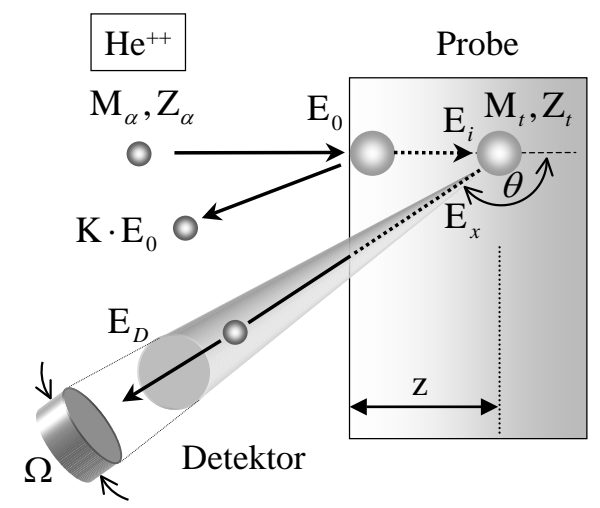

Abbildung 3.16: Prinzipskizze der Rutherford Backscattering Spectrometry (RBS).

gestreut. Der Silizium-Detektor ist in einem Winkel von $\theta=165^{\circ}$ angeordnet und bedeckt einen Raumwinkel $\Omega$ von 3,4 msr. Aufgrund der Abbremsung im Targetmaterial ist die Energie um einen gewissen Betrag vermindert, der durch den kinematischen Faktor beschrieben wird [86]

$$
K=\left[\frac{\sqrt{M_{t}^{2}-M_{\alpha}^{2} \sin ^{2} \theta}+M_{\alpha} \cos \theta}{M_{t}+M_{\alpha}}\right]^{1 / 2}
$$

Der Energieverlust beträgt dann:

$$
\Delta E=K \cdot E_{0}-E_{D}
$$

mit

$$
E_{D}=K\left(E_{0}-\Delta E(z)\right)-\Delta E(z / \cos \theta)
$$

Die Energie $E_{D}$ eines gestreuten $\alpha$-Partikels ist unter Verwendung der stopping power $d E / d x[86,123$ :

$$
E_{D}=K\left(E_{0}-\left.\int_{0}^{z} \frac{d E}{d x}\right|_{E} d x\right)-\left.\int_{0}^{z / \cos \theta} \frac{d E}{d x}\right|_{K E} d x
$$

$d E / d x$ gibt den Energieverlust pro zurückgelegter Strecke an. Eine weitere Größe ist die stopping cross section $\varepsilon$, die den Energieverlust pro Flächendichte angibt:

$$
\varepsilon=\frac{1}{N} \cdot \frac{d E}{d x} \quad, \quad \text { Einheit }(\varepsilon)=\frac{\mathrm{eV}}{10^{15} \mathrm{Atome} \cdot \mathrm{cm}^{2}}
$$

Für jedes Element $i$ ist $\varepsilon_{i}$ verschieden. Für eine Verbindung ist diese Größe nach der Braggschen Regel einfach die gewichtete Summe [124]

$$
\varepsilon_{A_{m} B_{n}}=m \cdot \varepsilon_{A}+n \cdot \varepsilon_{B}
$$

Für die stopping power gilt ein entsprechender Zusammenhang [123]:

$$
\frac{d E_{A_{m} B_{n}}}{d x}=N_{A_{m} B_{n}} \cdot\left(m \varepsilon_{A}+n \varepsilon_{B}\right)
$$

\subsubsection{Wirkungsquerschnitt}

Der Wirkungsquerschnitt ist definiert als [125, 126]:

$$
\sigma(\theta)=\frac{(\text { Zahl der nach } d \Omega \text { gestreuten Teilchen }) \cdot s^{-1}}{(\text { Zahl der gesamten einfallenden Teilchen }) \cdot\left(s \cdot m^{2}\right)^{-1}}
$$

Abb. (3.17) zeigt die relevanten Größen einer Streuung. Der Detektor befindet sich im Winkel $\theta$ zum 


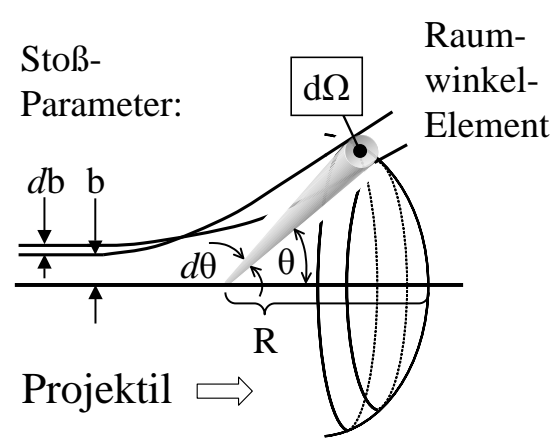

Abbildung 3.17: Streugeometrie und Parameter eines Teilchens.

einfallenden Strahl und deckt den Raumwinkel $d \Omega$ ab. Der differenzielle Wirkungsquerschnitt ist auf den Raumwinkel $d \Omega$ normiert:

$$
\sigma_{\text {diff }}=\frac{d \sigma(\theta)}{d \Omega}
$$

Für den Wirkungsquerschnitt im Fall der Rutherford-Streuung gilt [86]:

$$
\frac{d \sigma(\theta)}{d \Omega}=\left(\frac{Z_{\alpha} Z_{t} e^{2}}{4 E_{i}}\right)^{2} \frac{4}{\sin ^{4} \theta} \cdot \frac{\left[\sqrt{1-\left(M_{\alpha} / M_{t}\right)^{2} \sin ^{2} \theta}+\cos \theta\right]^{2}}{\sqrt{1-\left(M_{\alpha} / M_{t}\right)^{2} \sin ^{2} \theta}}
$$

Den totalen Wirkungsquerschnitt erhält man durch Integration über den Raumwinkel [123]:

$$
\sigma_{\mathrm{tot}}=\int_{\Omega} \frac{d \sigma(\theta)}{d \Omega} d \Omega
$$

Der Raumwinkel des Detektors ist gewöhnlicherweise klein, und so definiert man den Streuquerschnitt als

$$
\sigma=\frac{1}{\Omega} \cdot \int_{\Omega} \frac{d \sigma(\theta)}{d \Omega} d \Omega
$$

Die Gesamtanzahl der detektierten $\alpha$-Teilchen ist gegeben durch

$$
H=\sigma \Omega \cdot Q \cdot N t
$$

dabei ist $Q$ die Anzahl der einfallenden Teilchen, $N t$ ist die Anzahl der Targetatome pro Flächeneinheit und $\sigma$ gemäß Gl. (3.77). Bei bekanntem $\sigma$ und $\Omega$ kann man nun die physikalische Dicke $N \cdot t$ bestimmen. Für eine Verbindung $A_{m} B_{n}$ setzt sich $H$ additiv zusammen:

$$
H_{A_{m} B_{n}}=H_{A}+H_{B} \quad \text { mit } H_{A}=\sigma_{A} \Omega Q \cdot m \cdot H_{A_{m} B_{n}} t_{A} \quad \text { und } \quad H_{B}=\sigma_{B} \Omega Q \cdot n \cdot H_{A_{m} B_{n}} t_{B}
$$

So erhält man schließlich:

$$
\frac{H_{A}}{H_{B}}=\frac{\sigma_{A} \cdot \Omega Q \cdot m N_{A_{m} B_{n}} t_{A}}{\sigma_{B} \cdot \Omega Q \cdot n N_{A_{m} B_{n}} t_{B}}=\frac{\sigma_{A} \cdot m \cdot t_{A}}{\sigma_{B} \cdot n \cdot t_{B}}
$$

Über einen Umweg kann man das Verhältnis $t_{A} / t_{B}$ errechnen. Dazu führt man den Stopping-QuerschnittsFaktor $[\varepsilon]$ ein. Der Energieverlust aus Gl. (3.67) kann dann geschrieben werden als

$$
\Delta E=[\varepsilon] \cdot N t
$$

Analog gilt für die Elemente $A$ oder $B$ der Verbindung $A_{m} B_{n}$ :

$$
\Delta E^{A_{m} B_{n}}=[\varepsilon]_{A, B}^{A_{m} B_{n}} \cdot N^{A_{m} B_{n}} t_{A, B}
$$

Schreibt man Gl. 3.80 in dieser Notation um, so ergibt das

$$
\frac{H_{A}}{H_{B}}=\frac{\sigma_{A} \cdot m \cdot[\varepsilon]_{A}^{A_{m} B_{n}}}{\sigma_{B} \cdot n \cdot[\varepsilon]_{B}^{A_{m} B_{n}}}
$$


Die Stöchiometrie ist jetzt einfach zu berechnen, weil das Verhältnis in 1. Näherung $[\varepsilon]_{A}^{A_{m} B_{n}} /[\varepsilon]_{B}^{A_{m} B_{n}}=1$ gesetzt werden kann; der Fehler beträgt hierbei nur wenige Prozent 123 86]:

$$
\frac{n}{m}=\frac{H_{A} \cdot \sigma_{A}}{H_{B} \cdot \sigma_{B}}
$$

Zur Limitierung der Tiefen-Aufösung einer RBS-Messung tragen im Wesentlichen drei unterschiedliche Anteile bei:

1. Energie-Straggling $\Omega_{\text {strag }}$

2. Energie-Breite des auftreffenden $\alpha$-Strahls $\Omega_{\text {beam }}$

3. Auflösung des Detektors $\Omega_{\mathrm{det}}$

Abb. 3.18 zeigt ein Beispiel einer RBS-Messung an reinem Eisen. Die Steilheit der Vorderkante spiegelt

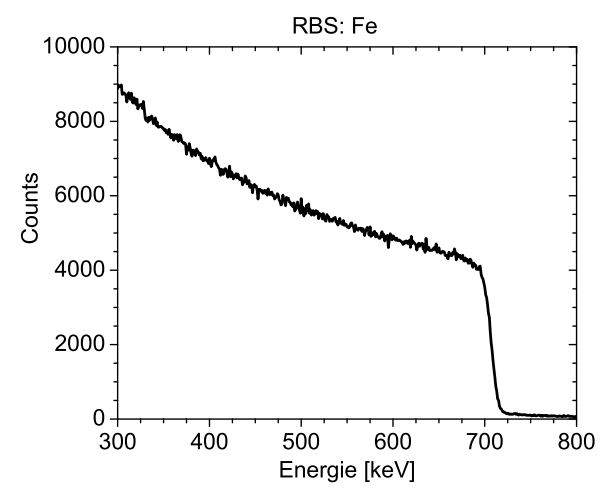

Abbildung 3.18: RBS-Spektrum von Eisen.

die Auflösung $\Omega_{\text {det }}$ des Halbleiter-Detektors wieder, die bei ca. $\Omega_{\text {det }}=12 \mathrm{keV}$ liegt. Die Energiebreite eines Partikel-Strahls am IONAS ist energie- und ladungsabhängig: Im Fall eines $900 \mathrm{keV} \alpha$-Strahls beträgt diese $\Omega_{\text {beam }}=79 \mathrm{eV}$ und besitzt eine gaussförmige Verteilung [122]. Beide Effekte sind apparativ bedingt im Gegensatz zum Energie-Straggling, das statistisch-physikalischer Natur ist. Durchdringen geladene Partikel Materie, dann sind diese den statistischen Schwankungen der Target-Atome unterworfen, so dass nicht alle Partikel denselben Energieverlust $E_{D}$ erleiden, sondern streuen mit einer Gaussverteilung um diesen Wert. Im Grenzfall hoher Ionen-Geschwindigkeiten, ist der Energievelust hauptsächlich durch elektronische Anregung

$$
\Omega_{\text {strag }}\left[\mathrm{keV}^{2}\right]=0,26 \cdot Z_{\alpha}^{2} Z_{t} \cdot N z\left[10^{18} \text { at. } / \mathrm{cm}^{2}\right]
$$

zu verzeichnen [127]. Für kleine Strahlenergien bzw. -geschwindigkeiten $E<75 Z_{t}[\mathrm{keV} / \mathrm{amu}]$ wurde Gl. (3.85) korrigiert 128]:

$$
\frac{\Omega_{\mathrm{LS}}}{\Omega_{\mathrm{strag}}}\left\{\begin{array}{cl}
0,5 \cdot f(x) & \text { für } \quad E<75 Z_{t}[\mathrm{keV} / \mathrm{amu}] \\
1 & \text { für } \quad E \geq 75 Z_{t}[\mathrm{keV} / \mathrm{amu}]
\end{array}\right.
$$

mit

$$
\begin{aligned}
f(x) & =1,36 \cdot x^{1 / 2}-0,016 \cdot x^{3 / 2} \\
x & =E[\mathrm{keV} / \mathrm{amu}] /\left(25 Z_{t}\right)
\end{aligned}
$$

\subsection{Mikrohärte-Messungen}

Die Härte eines Materials wird als Widerstand gegen das Eindringen einer härteren Spitze definiert [35]. In der Regel wird als Spitze eine Vickers-Diamant-Pyramide mit einem Öffnungswinkel von $\alpha=136^{\circ}$ verwendet (Abb. 3.19). Die Indenter-Tiefe $h$ wird dabei in Abhängigkeit von der Eindrück-Kraft $F$ der 
Spitze bestimmt. Die Abb. 3.19 zeigt eine typische $F$ - $h$-Kurve für poliertes, polykristallines Eisen. Die sog. Universalhärte $H U$ wird in $\mathrm{N} / \mathrm{mm}^{2}$ bzw. MPa gemessen und ist das Verhältnis der angewendeten Prüfkraft $F$ zu der Kontaktfläche $A$ zwischen Material und der Eindringtiefe des Diamanten. Die Messungen werden mit einem Fischerscope HV $100[129,130]$ durchgeführt, dessen Tiefenauflösung $<2$ nm und Kraftauflösung $\approx 20 \mathrm{nN}$ beträgt [131. Die maximale aufgewendete Kraft beträgt hier im Falle von Eisen und Edelstahl $1000 \mathrm{mN}$. Die Kontakt-Fläche $F$ der quadratischen Pyramide ist von der Eindringtiefe abhängig und beträgt:

$$
A(h)=4 h^{2} \cdot \frac{\sin \left(1 / 2 \cdot 136^{\circ}\right)}{\cos ^{2}\left(1 / 2 \cdot 136^{\circ}\right)}=26,429 \cdot h^{2}
$$

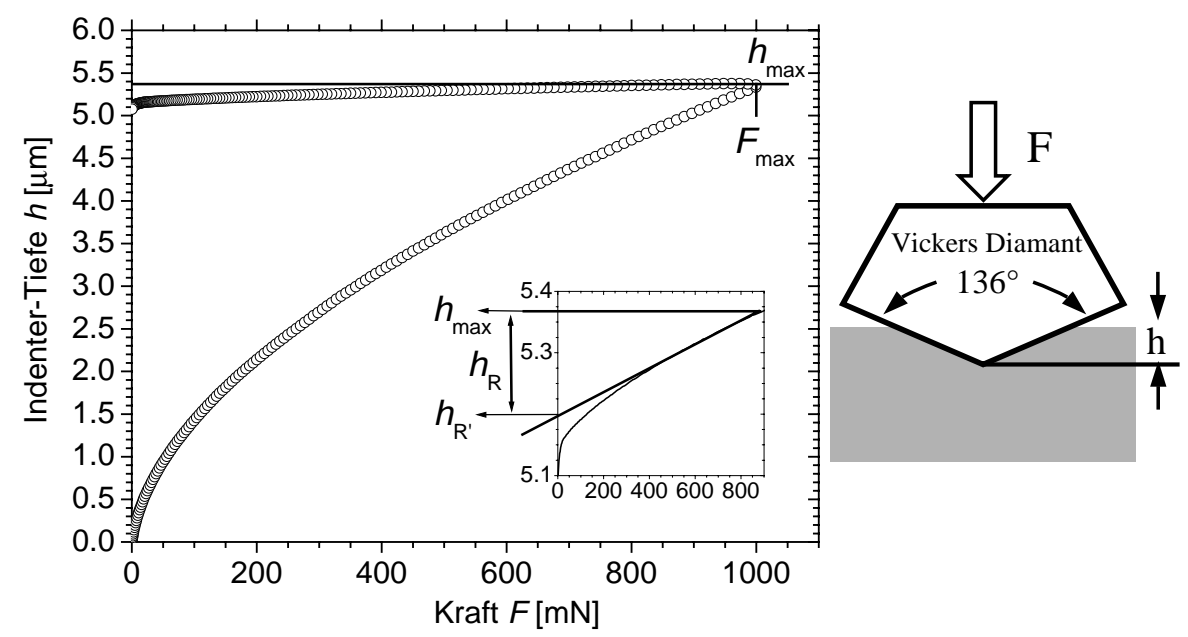

Abbildung 3.19: Eindring-Tiefe des Vickers-Diamanten in Abhängigkeit zur Kraft. Das Material ist polykristallines, auf $1 \mu \mathrm{m}$-poliertes Eisen. Im kleinen Bild sind die charakteristischen Größen der Messung dargestellt. Der rechte Teil zeigt die Geometrie des Nanoindenters.

Für die Universalhärte $H U$ ergibt sich demnach:

$$
H U=\frac{F}{A}=\frac{F}{26,429 \cdot h^{2}}
$$

Die obige Betrachtung gilt nur für den idealen Vickers-Diamanten. In der Realität liegt natürlich kein perfekter Diamant vor. Jede Abweichung von der idealen Geometrie führt zu z.T. großen Fehlern in der bestimmten Härte. Diese Fehler können aber durch Vergleich mit einer bekannten Härtekurve herausgerechntet werden. Als Referenz benutzt man hierbei ein 5mm dickes Si(100)-Substrat. Die Härte wird gemessen und entsprechend korrigiert, bis der reale Wert erreicht wird. Auf diese Weise erhält man eine Korrektur-Funktion, mit der dann alle weiteren Härteprofile errechnet werden. Nach [132 kann man aus der plastischen bzw. der elastischen Arbeit den E-Modul berechnen:

$$
\frac{E}{\left(1-\nu^{2}\right)}=\frac{\sqrt{\pi} \cdot F}{4 \cdot \tan \left(1 / 2 \cdot 136^{\circ}\right) \cdot h_{R} \cdot h_{R^{\prime}}}
$$

dabei ist $\nu$ die Poisson-Zahl, $h_{R^{\prime}}$ der Ordinatenabschnitt der Tangente am Anfang der Entlastungskurve und $h_{R}=h_{\max }-h_{R^{\prime}}$ die Differenz aus der maximalen Eindrücktiefe und $h_{R^{\prime}}$. Die relevanten Größen sind in Abb. 3.19 veranschaulicht.

Die $F$-h-Kurven werden durch konstante, schrittweise Erhöhung (150 Schritte) von $\sqrt{F}$ erhalten; die entsprechende Tiefe $h$ wird bei jedem Schritt registriert. Die Kraft wird jeweils für eine Zeitspanne von $0,1 \mathrm{~s}$ auf dem Wert gehalten und anschließend erhöht.

\subsection{Eigenspannungs-Bestimmung}

Während der Laser-Bestrahlung treten im Material wegen der enormen Aufheiz- und Abkühlraten hohe thermische und mechanische Belastungen auf, die schließlich im erstarrten Zustand fortbestehen, die sog. 
Eigenspannung (oder stress) $\sigma$. Die Spannungen verursachen eine Verzerrung des Gitters und verändern daher die Gitterparameter. Es gibt 2 Arten von Eigenspannungen (Abb. 3.20): Sind die Gitter-Parameter (c) normal zur Oberfläche gestaucht und die parallel $(a)$ zur Oberfläche gedehnt, so liegt Zugspannung (tensile stress) vor: $a_{t}>a$ und $c_{t}<c$ mit $\sigma>0$. Im umgekehrten Fall ist es Druckspannung (compressive stress): $a_{c}<a$ und $c_{c}>c$ mit $\sigma<0$. Die Röntgen-Reflexe sind aufgrund des Stresses in charakteristischer

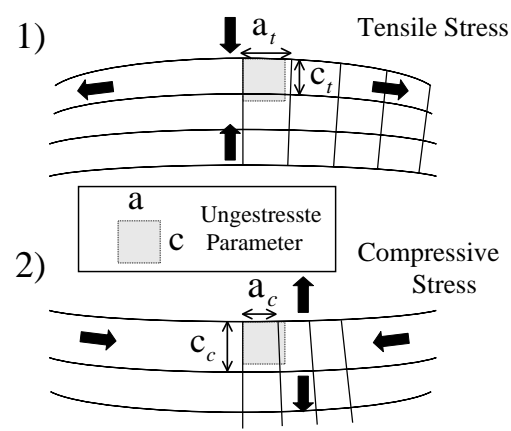

Abbildung 3.20: Veranschaulichung von Zug-(tensile) und Druckspannung (compressive stress).

Weise gegenüber den 2 $\theta$-Reflexen des spannungslosen (ungespannten) Gitters verschoben. XRD-Spektren mit festem Einfallswinkel erlauben die Bestimmung der Eigenspannungs- und Gitterparameter, da der Streuwinkel für jeden Reflex einer Phase verschieden ist (s. unten). Die zugrundeliegende Formel lautet nach Prévey [133]:

$$
\frac{d-d_{0}}{d_{0}}=\frac{\Delta d}{d_{0}}=(1+\nu)\left[\frac{\sigma}{E}\right] \sin ^{2}(\Phi)-2 \nu\left[\frac{\sigma}{E}\right]
$$

dabei sind: $\nu$ die Poisson-Zahl, die auf 0,3 (liegt im Bereich von Eisen und Gußeisen [3]) festgesetzt ist, $E / \sigma$ das gesuchte Verhältnis von Elastizitätsmodul zu Eigenspannung. In Grazing-Incidence-XRD Geometrie ist $\Phi=\theta-\alpha$ der Winkel zwischen der Oberflächennormalen und dem Streuvektor, $\alpha$ ist der Einfallswinkel.

Zur Analyse werden weiterhin die gemessenen $d$ und die (noch unbekannten) spannungslosen $d_{0}$ Ebenenabstände benötigt. Die gemessenen Werte erhält man einfach durch die Bragg-Gleichung:

$$
d=\frac{\lambda}{2 \sin \theta}
$$

Die genauen Peak-Positionen wurden mit dem Program WinFit! [134] durch Anpassen einer Pearson-VIIProfil-Funktion P VII ermittelt:

$$
P V I I=\frac{1}{H_{<\mathrm{hkl}>}} \cdot \frac{\Gamma(m)}{\Gamma(m-0,5)} \cdot \frac{2 \sqrt{2^{1 / m}-1}}{\sqrt{\pi}} \cdot\left[1+4\left(2^{1 / m}-1\right)\left(\frac{\left(2 \theta_{i}-2 \theta_{<\mathrm{hkl}>}\right)^{2}}{H_{<\mathrm{hkl}>}^{2}}\right)\right]^{-m}
$$

mit: $H_{<\mathrm{hkl}}>$ ist die Halbwertsbreite des Bragg-Reflexes $\theta_{<\mathrm{hkl}}>$ mit den korrespondierenden Millerindizes $<\mathrm{hkl}>, \Gamma(\ldots)$ die Gamma-Funktion, $\theta_{i}$ die gemessene Reflexlage und $m$ der Form-Faktor, der die Flankenform bestimmt: $m=1$ entspricht einem Lorentzprofil, ab $m>10$ wird das Gauss-Profil angenähert; für Röntgenspektren beträgt in der Regel 1,5<m<2. Dieses Profil gibt die beste Übereinstimmung zwischen beobachteten und gefitteten XRD-Spektren [135, 136]. Die aufgrund der Eigenspannung verursachten Verschiebungen der Bragg-Positionen können leicht berechnet werden:

$$
d=\frac{\lambda}{2 \sin \theta} \quad \Rightarrow \quad \Delta d=d-d_{0}=\frac{\partial}{\partial \theta}\left(\frac{\lambda}{2 \sin \theta}\right) \Delta \theta=-\frac{d_{0}}{\tan \theta} \Delta \theta
$$

und somit

$$
\Delta \theta=\theta-\theta_{0}=-\tan \theta \frac{\Delta d}{d_{0}}
$$

$d_{0}$ ist durch die Gitterparameter $a_{0}, c_{0}$ des hexagonalen Kristallsystems und den $<\mathrm{hkl}>$ eindeutig gegeben [109]:

$$
\frac{1}{d_{0}}=\frac{4}{3} \frac{\left(h^{2}+h k+k^{2}\right)}{a_{0}^{2}}+\frac{l^{2}}{c_{0}^{2}}
$$




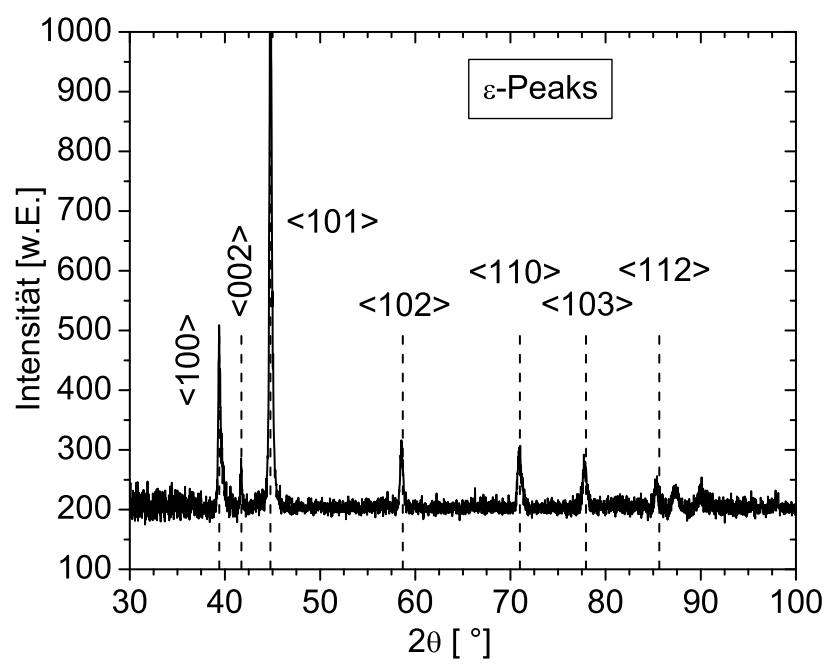

Abbildung 3.21: Indizierte Reflexe der bei $\mathrm{p}\left(\mathrm{CH}_{4}\right)=0,1$ bar und $11 \times 12$ Pulsen bestrahlten Probe, die auch als $\varepsilon$-Referenz dient.

Die Abb. 3.21 zeigt das XRD-Spektrum der $\varepsilon$-Referenz. Die entsprechende $<$ hkl $>\Leftrightarrow 2 \theta$ Zuordnung wird mit der Datenbank PCPDFWIN vorgenommen [34]. Mit diesen Gleichungen ergibt sich eine Formel, mit der die 3 gesuchten Parameter $a_{0}, c_{0}$ und $(\sigma / E)$ angefittet werden können:

$$
\sin ^{2}\left(\theta-\tan \theta\left[(1+\nu)\left(\frac{\sigma}{E}\right) \sin ^{2}(\Phi)-2 \nu\left(\frac{\sigma}{E}\right)\right]\right)-\left(\frac{\lambda^{2}}{3} \frac{\left(h^{2}+h k+k^{2}\right)}{a_{0}^{2}}+\frac{l^{2}}{c_{0}^{2}}\right)=0
$$

In Abb. 3.22 ist der Plot $\Delta d / d_{0}$ vs. $\sin ^{2} \Phi$ gemäß Gl. 3.90 skizziert, die Werte sind in Tab. 3.5 zusätzlich mit den Literaturdaten aufgeführt. Die Druckspannung $\sigma$ beträgt ca. 1,8\% des Elastizitätmoduls der

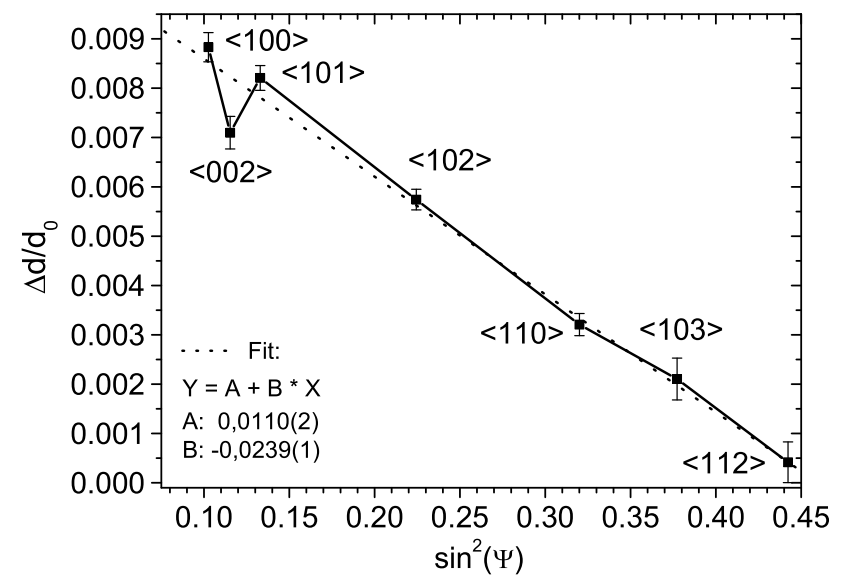

Tabelle 3.5: Vergleich der gefitteten mit den Literaturwerten.

\begin{tabular}{|c||l|c|}
\hline Parameter & gefittet & Literatur \\
\hline \hline $\mathrm{a}[\AA]$ & $2,665(1)$ & 2,754 \\
\hline $\mathrm{c}[\AA]$ & $4,358(1)$ & 4,349 \\
\hline$(1+\nu) \cdot(\sigma / E)$ & $-0,0239(1)$ & - \\
$\nu=0,3 \rightarrow \sigma / E$ & $-0,0184(1)$ & - \\
\hline
\end{tabular}

Abbildung 3.22: Auftragung $\Delta d / d_{0}$ gegen $\sin ^{2} \Phi$.

$\varepsilon$-Phase, dessen Literaturwert jedoch nicht zu ermitteln war.

\subsection{Sonstige Messmethoden}

In diesem Abschnitt werden weitere Messmethoden kurz vorgestellt, die nicht in großem Maße angewandt wurden.

\subsubsection{Magneto-Optischer Kerr-Effekt (MOKE)}

Die wesentliche Eigenschaft des Magneto-Optischen Effekts beruht auf der optischen Anisotropie, die durch die Magnetisierung der Probenoberfläche herbeigeführt wird [137 138]. Arbeitet man bei Transmission von 
Licht durch Materie, so spricht man vom Magneto-Optischen Faraday-Effekt, bei Reflexion des Lichts an der Oberfläche vom Magneto-Optischen Kerr-Effekt (MOKE).

Die Polarisationsrichtung und die Elliptizität des transmittierten bzw. reflektierten Lichtes ändert sich dabei in Abhängigkeit von der Polarisation des einfallenden Lichtes und der Proben-Magnetisierung. Diese Änderung wird als sog. Kerr-Rotation bezeichnet. Im Experiment wurde linear-polarisiertes Licht eines He-Ne-Lasers benutzt. Die Polarisationsebene des reflektierten (bzw. transmittierten) Lichtes wird in diesem Fall gegenüber der Polarisationsebene des einfallenden Strahls um einen Winkel $\delta$ gedreht. Es gibt 3 Arten des Kerr-Effektes, die sich in Orientierung der Magnetisierungsvektors in der Probe und in der Einfallsebene des Lichtes unterscheiden:

1) Polarer Kerr-Effekt: Die Magnetisierungrichtung ist senkrecht zur Probenoberfläche, jedoch parallel zur Einfallsebene des Lichts.

2) Longitudinaler Kerr-Effekt: Die Magnetisierungrichtung liegt parallel zur Probenoberfläche und zur Einfallsebene des Lichts.

3) Transversaler Kerr-Effekt: Die Magnetisierungsrichtung liegt auch hier parallel zur Probenoberfläche, aber senkrecht zur Einfallsebene des Lichts.

Theoretisch werden magneto-optische Effekte mithilfe des dielektrischen Tensors $\hat{\varepsilon}$ beschrieben. Im isotropen Medium gilt

$$
\boldsymbol{D}=\hat{\varepsilon} \cdot \boldsymbol{E},
$$

$\hat{\varepsilon}$ ist eine $3 \times 3$-Diagonalmatrix. Bei zusätzlichem, äußerem magnetischen Feld bzw. zusätzlicher Magnetisierung der Probe kommen Nichtdiagonalelemente hinzu, die durch Spin-Bahn-Wechselwirkung verursacht werden. Bei Vernachlässigung weiterer optischer Anisotropien und Effekte höherer Ordnung (sog. VoigtEffekt) kann $\hat{\varepsilon}$ in einen isotropen (Diagonalelemente, $\varepsilon_{i i}$ ) und einen anti-symmetrischen Anteil (NichtDiagonalelemente, $\varepsilon_{i j}$ ) zerlegt werden, so dass das Dielektrische Gesetz (Gl. 3.97) folgendermaßen geschrieben werden kann:

$$
\hat{\boldsymbol{\varepsilon}}=\varepsilon \cdot[\hat{\mathbf{1}}+i Q(\boldsymbol{m} \times \boldsymbol{E})] \quad \Rightarrow \quad \boldsymbol{D}=\varepsilon \cdot[\boldsymbol{E}+i Q(\boldsymbol{m} \times \boldsymbol{E})]
$$

$\boldsymbol{m}$ ist der Richtungsvektor der Magnetisierung, $\boldsymbol{E}$ das elektrische Feld, $Q$ beschreibt die (Voigt-)Materialkonstante, die die magneto-optische Rotation der Polarisationsebene beschreibt; sie ist in guter Näherung proportional zur Magnetisierung $\boldsymbol{m}$. Zeigt $\boldsymbol{m}$ in 3-Richtung und sind die 3-Komponenten von $i Q(\boldsymbol{m} \times \boldsymbol{E})$ in $\varepsilon_{i j}$ zusammengefasst, so ergibt sich für den Tensor $\hat{\varepsilon}$ :

$$
\hat{\boldsymbol{\varepsilon}}=\left(\begin{array}{ccc}
\varepsilon_{11} & \varepsilon_{12} & 0 \\
\varepsilon_{21} & \varepsilon_{22} & 0 \\
0 & 0 & \varepsilon_{33}
\end{array}\right) \approx\left(\begin{array}{ccc}
\varepsilon_{11} & \varepsilon_{12} & 0 \\
-\varepsilon_{12} & \varepsilon_{11} & 0 \\
0 & 0 & \varepsilon_{11}
\end{array}\right),
$$

wobei nun $\varepsilon \propto \boldsymbol{m}$. Die Näherung in obiger Gl. (3.99) gilt hier nur für kubische Systeme. Daraus ergibt sich der Zusammenhang zwischen Winkel $\delta$ und Magnetisierung $\boldsymbol{m}$ :

$$
\delta \propto \boldsymbol{m}
$$

Bei den Messungen wurde der longitudinale Kerr-Effekt ausgenutzt. Eine detaillierte Beschreibung des Versuchsaufbaus und der Durchführung ist bei [139] zu finden.

\subsubsection{Raman-Spektroskopie}

Beim Raman-Effekt handelt es sich um einen Zwei-Photonen-Prozess, bei dem das einfallende Photon $\omega$ inelastisch gestreut wird, $\omega_{m}$ stellt dann die Frequenz des erzeugten bzw. vernichteten Phonons dar 140 141.

Wird ein Molekül mit der Frequenz $\omega_{0}$ bestrahlt, so treten im Streulicht neben der unverschobenen Linie (Rayleigh-Streuung) seitlich angeordnete, symmetrische Linien mit der Frequenz $\omega_{0} \pm \Omega_{i}$ auf, die sog. Raman-Streuung. Diese Energien $\hbar \cdot \Omega_{i}$ entsprechen den Rotations- und Schwingungsniveaus des streuenden Moleküls. Beschrieben wird der Raman-Effekt durch ein oszillierendes Dipolmoment

$$
\boldsymbol{\mu}_{\text {ind }}=\alpha \boldsymbol{E},
$$


das durch die einfallende elektromagnetische Welle $\boldsymbol{E}=\boldsymbol{E}_{0} \cos \omega t$ induziert wird. Das Gesamtdipolmoment ergibt sich mit einem permanenten Dipolmoment zu

$$
\boldsymbol{\mu}=\boldsymbol{\mu}_{0}+\alpha \boldsymbol{E}
$$

Für kleine Auslenkungen aus der Gleichgewichtslage $R_{g}$ kann man das Dipolmoment und die Polarisierbarkeit in eine Taylorreihe entwickeln, da diese Größen vom Kernabstand und den Elektronenkoordinaten zusammenhängen. $A_{m}$ und $\omega_{m}$ sind Amplitude und Frequenz der Molekülschwingung, $\alpha(0)$ die Polarisierbarkeit im Gleichgewichtsabstand:

$$
\begin{aligned}
\boldsymbol{\mu}(t)= & \boldsymbol{\mu}_{0}+\left(\frac{\partial \boldsymbol{\mu}}{\partial R}\right)_{R_{g}} A_{m} \cos \omega_{m} t+\alpha(0) \boldsymbol{E}_{0} \cos \omega t \\
& +\frac{\boldsymbol{E}_{0}}{2}\left(\frac{\partial \alpha}{\partial R}\right)_{R_{g}} A_{m}\left[\cos \left(\omega_{0}-\omega_{m}\right) t+\cos \left(\omega_{0}+\omega_{m}\right) t\right]
\end{aligned}
$$

Für den Raman-Effekt ist es nun wesentlich, dass die Polarisierbarkeit mit der Energieänderung variiert $\partial \alpha / \partial R \neq 0$, während die Änderung des Dipolmomentes $\partial \boldsymbol{\mu} / \partial R \neq 0$ für die Infrarot-Absorptions-Spektren relevant ist. $\left(\omega_{0}-\omega_{m}\right)$ wird als ,Stokes“-Linie, $\left(\omega_{0}+\omega_{m}\right)$ als „Anti-Stokes"-Linie bezeichnet, die nur auftritt, wenn sich das Molekül vor der Streuung im angeregten Zustand befindet.

Die Messungen wurden im IV. Physikalischen Institut der Universität Göttingen durchgeführt. Angeregt wurde mit einem 514,5 nm Ar-Laser. Die Streustrahlung wurde mit einer CCD-Kamera im Bereich von ca. $1175-1750 \mathrm{~cm}^{-1}$ detektiert.

\subsection{Laserbearbeitung}

ARMCO-Eisen der Reinheit 99,85\% [142] wird in Stangen mit $25 \mathrm{~mm}$ Durchmesser ausgeliefert und wurde in Scheibchen mit 1,5 mm Dicke zersägt. Der austenitische Edelstahl [143] wurde aus $1 \mathrm{~mm}$ dicken Folien $\left(15 \times 15 \mathrm{~cm}\right.$ Seitenlänge) in Plättchen zu je $15 \times 15 \mathrm{~mm}^{2}$ geschnitten. Die Roh-Proben werden der Reihe nach mit 1200er, 2400er und 4000er SiC-Schleifpapier nassgeschliffen. Im letzten Schritt poliert man sie mit $1 \mu \mathrm{m}$-Diamantpaste und entfettet mit Aceton und Ethanol. Die Rauhigkeit beträgt dann $R_{a}=15(4) \mathrm{nm}$ [144].

Die Laser-Bestrahlung erfolgt mit einem Siemens XP2020 Excimer-Laser. Die Daten sind in Tab. 3.6 zusammengefasst: Die Proben werden in einer Aluminium-Kammer plaziert, die vor der Gasbefüllung

Tabelle 3.6: Kenndaten des Siemems XP2020 Lasers und des fokussierten Strahls.

\begin{tabular}{|l|c|}
\hline \multicolumn{1}{|c|}{ Parameter } & Wert \\
\hline \hline Art des Gases & XeCl \\
\hline Wellenlänge $\lambda[\mathrm{nm}]$ & 308 \\
\hline Max. Pulsenergie $E[\mathrm{~J}]$ & 2 \\
\hline Pulsdauer (FWHM) $t_{p}[\mathrm{~ns}]$ & 55 \\
\hline Max. Pulsfrequenz $f_{p}[\mathrm{~Hz}]$ & 10 \\
\hline Spotgröße im Focus $x \cdot y\left[\mathrm{~mm}^{2}\right]$ & $\begin{array}{c}\text { Raw Beam: } \\
=4,5 \times 5,5 \\
\text { homogenisiert: } \\
=5 \times 5\end{array}$ \\
\hline
\end{tabular}

bis zu einem Restdruck von $p_{R} \approx 10^{-6}$ bar evakuiert wird. Die Kammer wird anschließend mit Methan (Reinheit $\approx 99,85 \%$ ) befüllt, die Drücke variieren von 0,1 bis 10 bar. Abb. 3.23 zeigt den Versuchsaufbau der Laser-Kammer. Die Laserstrahlung tritt von oben durch ein Quarz-Fenster in die Kammer ein. Der Laserstrahl kann mit 2 verschiedenen Linsen fokussiert werden.

a) Eine Quarz-Sammellinse der Brennweite $f_{\mathrm{rb}} \simeq 190 \mathrm{~mm}$ : Das Intensitätsprofil des fokussierten Strahls ist jedoch inhomogen, man spricht daher von Raw-Beam. 


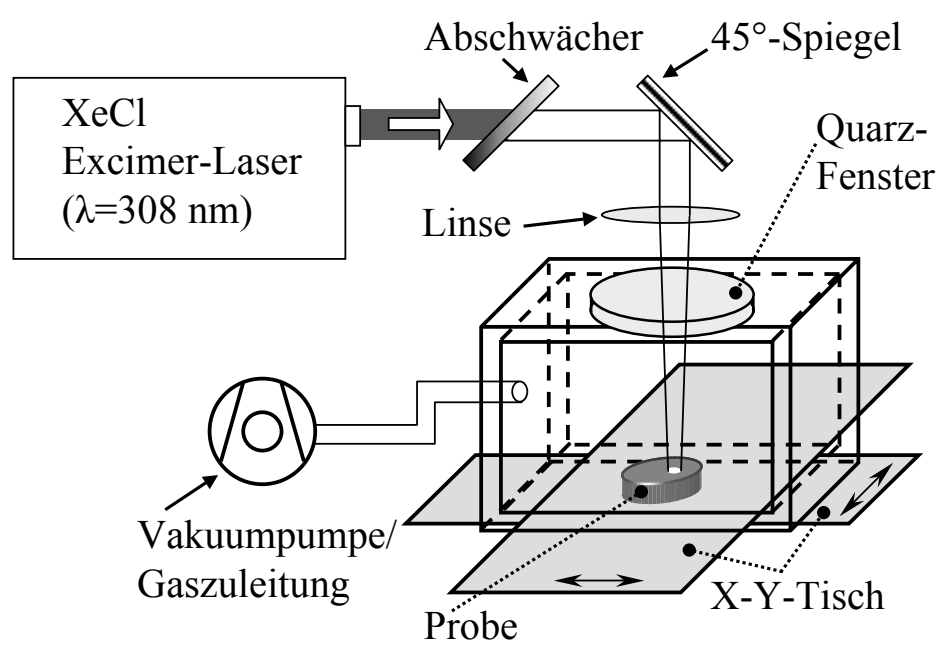

Abbildung 3.23: Prinzipskizze des experimentellen Aufbaus der Laserkammer und des computergesteuerten $x-y$ Tisches.

b) Eine „Fly-Eye“-oder Homogenisator-Linse der Brennweite $f_{\text {hom }} \simeq 140 \mathrm{~mm}$. Diese Linse besteht u.a. aus mehreren gekreuzten, zylindrischen Linsen. Deren Intensitätsprofile überlappen sich und das daraus resultierende Top-Hat-Profil ist sehr homogen [131].

Fast alle Bestrahlungen wurden mit der Homogenisator-Linse durchgeführt. Im Fokus beträgt die Spotfläche ca. $25 \mathrm{~mm}^{2}$. Die Energiedichte $H=E / A$ wird mit einem pyroelektrischen SpectroLas ${ }^{\bigodot_{-}}$Joule-Meter bestimmt. Da die Fläche $A$ im Focus nicht mehr veränderlich ist, verändert man die Energiedichte $H$, indem die Energie $E$ des Lasers mit einem Abschwächer reguliert wird. Je nach Anstellwinkel wird der gewünschte Anteil der Strahlung transmittiert. Die Energie des Lasers kann man direkt am Gerät durch Variation der Hochspannung in der Vorionisations-Röntgenkammer regeln.

Die Proben kann man auf 2 Arten bestrahlen. Bei Einzelspots wird die gleiche Stelle des Materials mit der entsprechenden Anzahl beschossen. Beim sog. Mäandrieren wird die Oberfläche vom Laser abgerastert. In Abb. 3.24 ist der Übergang von Einzelspot- zum Mäanderverfahren skizziert: Die Kammer steht dazu

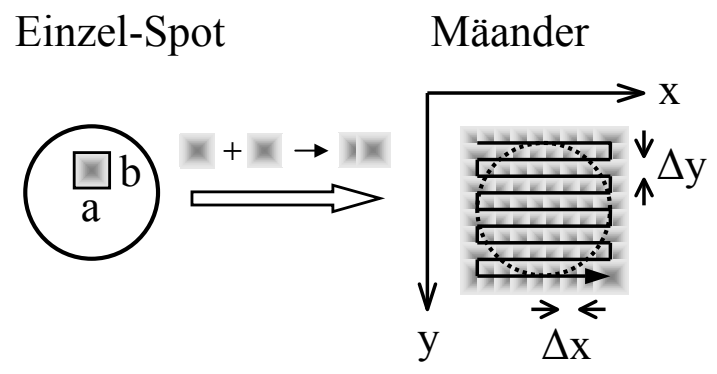

Abbildung 3.24: Unterschied zwischen Einzelpuls- und Mäander-Verfahren.

auf einem computergesteuerten $x$ - $y$-Tisch. Durch geeigneten Einzelspot-Überlapp wird erreicht, dass jedes Flächenelement effektiv mit $n \cdot m$ Pulsen bedeckt wird: Dazu bewegt sich der Tisch nach jedem Puls mäanderförmig in $x$-Richtung um $\Delta x=a / n$ und um $\Delta y=b / m$ in $y$-Richtung, was im Gegensatz zu Einzelpulsen als Bestrahlung mit , $n \times m^{\prime \prime}$ Pulsen bezeichnet wird. Um eine beliebige Probe der Größe $x \cdot y \mathrm{~mm}^{2}$ mit der gewünschten Anzahl Pulsen/Fläche zu bedecken, müssen diese Achsenvorschübe $\Delta x$ und $\Delta y$ mit der Spotgröße $a \cdot b$ und der Pulsfrequenz $f_{p}$ verrechnet werden. 


\section{Kapitel 4}

\section{Laser-Materie-Wechselwirkungen}

Für die Laser-Bearbeitung ist es wichtig, die Mechanismen zu kennen, durch die die Laserenergie eingekoppelt und wie diese durch Relaxationsprozesse an das Gitter bzw. die Elektronen übertragen wird. In Metallen wird die Laserstrahlung durch die Elektronen des Leitungsbandes absorbiert, die auch für die optischen Eigenschaften verantwortlich sind 13,11. Die Elektron-Elektron-Stoßzeiten $\tau_{\text {ee }}$ liegen im Bereich von $10^{-14}-10^{-12} \mathrm{~s}$, während die Elektron-Phonon-Relaxationszeit $\tau_{\text {ep }}$ zwischen $10^{-12}$ und $10^{-10}$ $\mathrm{s}$ liegt. Letztere ist die typische Zeit $\tau_{\text {therm }}$, in der die Energie thermalisiert. Diese Zeit ist sehr klein im Vergleich zur Pulsdauer des Lasers mit $\tau_{p}=55$ ns. In diesem Fall ist $\tau_{p}$ die Relaxationszeit $\tau_{\mathrm{i}}$, die die Laser-Prozess-Initialisierung charakterisiert. Allgemein gilt, wenn $\tau_{\text {therm }} \ll \tau_{\mathrm{i}}$, liegt ein thermischer Prozess vor, d.h. der Laser ist als eine rein thermische Strahlungsquelle anzusehen [11].

\subsection{Elektronische Eigenschaften der Metalle}

Das elektromagnetische Feld der auftreffenden Strahlung veranlasst das Elektron zu erzwungenen Schwingungen gleicher Frequenz. Phase und Amplitude ergeben sich aus der Schwingungsgleichung [145]:

$$
m \frac{d^{2} x}{d t^{2}}+m \gamma \frac{d x}{d t}=-e \cdot E_{0} e^{-i \omega t}=-e \cdot E(t)
$$

$e$ und $m$ sind Elektronenladung und -Masse, $\gamma$ ist die Dämpfungskonstante und $E_{0}$ die Amplitude der einfallenden Strahlung. Im Gegensatz zu Systemen mit gebundenen Elektronen-Zuständen fehlt hier der Eigenfrequenzterm $\omega_{0}$. Die Differenzial-Gleichung besitzt die Lösung:

$$
x(t)=\frac{e}{m} \cdot \frac{1}{\omega^{2}+i \gamma \omega} \cdot E(t)
$$

Für $N$ Elektronen und die Polarisation $P(t)=N \cdot p(t)=-N e \cdot x(t)$ erhält man

$$
P(t)=-\frac{N e^{2}}{m} \cdot \frac{1}{\omega^{2}+i \gamma \omega} \cdot E(t)
$$

Der Zusammenhang von $P(t)$ und $E(t)$ ist über $P(t)=\varepsilon_{0} \chi_{e} E(t)$ gegeben, $\chi_{e}$ ist die dielektrische Suszeptibilität [105]. Mit der Definition der komplexen dielektrischen Funktion $\varepsilon(\omega)=1+\chi_{e}$, die die Antwort auf die eingestrahlte Welle beschreibt, folgt:

$$
P(t)=\varepsilon_{0} \chi_{e} E(t) \quad \Rightarrow \quad \varepsilon(\omega)=1-\left(\frac{N e^{2}}{m \varepsilon_{0}}\right) \frac{1}{\omega^{2}+i \gamma \omega}=\varepsilon^{\prime}(\omega)+i \varepsilon^{\prime \prime}(\omega)
$$

Für vernachlässigbare Dämpfung $\gamma \ll \omega \operatorname{kann} \varepsilon^{\prime \prime}(\omega) \approx 0$ gesetzt werden:

$$
\varepsilon^{\prime}(\omega)=1-\frac{N e^{2}}{m \varepsilon_{0}} \cdot \frac{1}{\omega^{2}}=1-\frac{\omega_{p}^{2}}{\omega^{2}} \quad \text { mit } \quad \omega_{p}=\sqrt{\frac{N e^{2}}{m \varepsilon_{0}}}
$$


mit der sog. Plasmafrequenz $\omega_{p}$. Für Frequenzen $\omega \ll \omega_{p}$ ist $\varepsilon^{\prime}(\omega)<0$, somit wird der Brechungsindex $m$ imaginär, der über

$$
m(\omega):=n-i k=\sqrt{\varepsilon^{\prime}(\omega)}
$$

gegeben ist [146]. Als Konsequenz findet eine starke Dämpfung der Welle im Medium statt. Der Absorptionskoeffizient ist direkt proportional zum Imaginärteil des Brechungsindex':

$$
\alpha=\frac{4 \pi k}{\lambda}
$$

Im UV-Bereich gilt für Metalle: $\alpha \approx 10^{6} \mathrm{~cm}^{-1}$. Der absorbierte Teil $I_{a}$ der eingestrahlten Laser-Intensität $I_{0}$ nimmt mit der Tiefe exponentiell nach dem Beerschen Absorptionsgesetz [147] ab:

$$
I(z)=I_{a} \cdot e^{-\alpha z} \quad \text { mit } I_{a}=A \cdot I_{0}=(1-R) \cdot I_{0} \quad,
$$

dabei ist $A$ die Absorptivität und $R$ die Reflektivität mit $A+R=1$. Für Eisen gilt bei der Wellenlänge $\lambda=308 \mathrm{~nm}: \alpha=8,4 \cdot 10^{7} \mathrm{~m}^{-1}[148$ und $A=1-R=0,59$ [146]. Die optische Eindringtiefe beträgt demnach $\alpha^{-1}=l_{\alpha} \sim 12 \mathrm{~nm}$. In die zeitliche Entwicklung der Intensität $I_{0}$ geht die Form des Laserpulses ein und kann in folgender Weise angegeben werden [24]:

$$
I_{0}(t)=\frac{H \cdot t}{\sigma^{2}} \cdot \exp \left(-\frac{t^{2}}{2 \sigma^{2}}\right)
$$

$H$ ist die Energiedichte des Lasers $\left[\mathrm{J} / \mathrm{cm}^{2}\right]$ und $t_{p}=1,6 \sigma$ die Pulsdauer des Lasers. Im Inset der Abb. 4.1 ist die Pulsform eingezeichnet.

\subsection{Wärmeleitungs-Gleichung}

Auf makroskopischer Ebene kann die Antwort des Materials auf die Laserstrahlung mit der Wärmeleitungsgleichung ausgedrückt werden. Die thermische Diffusivität von (ARMCO-)Eisen bei $300 \mathrm{~K}$ beträgt $\kappa=0,206 \mathrm{~cm}^{2} / \mathrm{s}[149]$ und im geschmolzenen Zustand $\kappa<0,07 \mathrm{~cm}^{2} / \mathrm{s}$ [150]. Die thermische Diffusionlänge berechnet sich zu $\ell_{D}=2 \sqrt{\kappa \tau_{p}} \approx 1,2 \mu \mathrm{m}$, was erheblich kleiner ist als die Ausdehnung des Laserspots. Daher kann das Temperaturfeld mit der eindimensionalen Wärmeleitungsgleichung errechnet werden [151]. In der allgemeinen dreidimensionalen Gleichung nimmt man die Ersetzungen $\mathbf{r} \leftrightarrow z$ und $\nabla_{\mathbf{r}} \leftrightarrow \partial / \partial z$ vor.

$$
\begin{aligned}
\rho(T) c_{p}(T) \cdot \frac{\partial T(z, t)}{\partial t} & =\frac{\partial}{\partial z}(\Phi(z, t))+Q(z, t) \\
\Rightarrow \quad \rho(T) c_{p}(T) \cdot \frac{\partial T(z, t)}{\partial t} & =\frac{\partial}{\partial z}\left(\lambda(T) \frac{\partial T(z, t)}{\partial z}\right)+I_{0}(t)[1-R(T)] \alpha \cdot e^{-\alpha(T) z}
\end{aligned}
$$

wobei $\rho\left[\mathrm{kg} / \mathrm{m}^{-3}\right]$ die Dichte ist, $T[\mathrm{~K}]$ ist die Temperatur, $c_{p}\left[\mathrm{JK}^{-1} \mathrm{~kg}^{-1}\right]$ die spezifische Wärmekapazität, $\lambda$ die thermische Leitfähigkeit $[\mathrm{W} /(\mathrm{K} \cdot \mathrm{m})]$ und $t[\mathrm{~s}]$ die Zeit beginnend mit dem Laserimpuls. $\Phi(z, t)$ ist der Wärmefluss. Der sog. Quell-Term $Q(z, t)$ kommt durch die Lasereinkopplung in das Material zustande. Mit der Wärmeleitungsgleichung können die Temperaturprofile sowohl der flüssigen als auch der festen Phase berechnet werden. Die Lösung der G1. 4.10 erfordert wegen ihrer Nicht-Linearität numerische Methoden, was in Abschnitt 4.3 näher besprochen wird.

Auf der Oberfläche entstehen während des Laserprozesses zwei Phasen-Grenzflächen: Dampf-Flüssigkeit $(v l)$ und Flüssigkeit-Festkörper $(l s)$. Zur Lösung müssen noch Randbedingungen miteinbezogen werden. Die Schmelzfront wird als Stefan's Problem behandelt 152 153]: Die Grenzfläche wird dabei als isotherm betrachtet, die die Schmelztemperatur $T_{m}$ hat. Die Fortpflanzungsgeschwindigkeit hängt vom thermischen Gradienten der jeweiligen Grenzfläche ab und kann für die $l s$-Phasenfront durch folgende Gleichung beschrieben werden [154,13]:

$$
-\lambda_{l} \frac{\partial T}{\partial z}+\lambda_{s} \frac{\partial T}{\partial z}=v_{l s} \rho L_{m}
$$


dabei sind: $L_{m}$ die Schmelzenthalpie, $\lambda_{l, s}$ die Wärmeleitfähigkeit der festen $s$ bzw. der flüssigen $l$ Phase und $v_{l s}$ die Geschwindigkeit der Schmelzfront. Entsprechendes gilt für die vl-Phasenfront [155, 156]

$$
-\lambda_{l} \frac{\partial T}{\partial z}+A \cdot I_{0}=v_{v l} \rho L_{v}
$$

$v_{v l}$ die Geschwindigkeit der Dampf-Flüssigkeitsgrenzfläche und $L_{v}$ die Verdampfungsenthalpie. $A \cdot I_{0}$ ist der Anteil der eingekoppelten Laserenergie in die geschmolzene Metalloberfläche. Die Ableitungen sind dabei an den Positionen der Phasengrenzen zu nehmen.

Wird die Schmelztemperatur erreicht, so dampft das Metall mit der Ablationsrate

$$
\frac{\partial z_{D}}{\partial t}=\frac{p_{D}(T)}{\rho \sqrt{2 \pi k_{B} T / m}}
$$

ab [146]. Dabei ist $p_{D}(T)$ der Dampfdruck, der durch die Clausius-Clapeyron-Gleichung gegeben ist

$$
p_{D}(T)=p_{0} \cdot \exp \left[\frac{L_{v}}{R}\left(\frac{1}{T_{s d}}-\frac{1}{T}\right)\right]
$$

Die Atome, die in die Gasphase abdampfen, befinden sich nicht im thermischen Gleichgewicht; sie weisen eine Semi-Maxwell'sche Geschwindigkeitsverteilung auf, d.h. die Geschwindigkeitsverteilung entspricht zwar der Oberflächentemperatur, aber die Geschwindigkeitsvektoren weisen alle von der Oberfläche weg. Durch gegenseitige Stöße jedoch erreichen sie innerhalb der sog. Knudsen-Schicht, die wenige freie Weglängen (einige $\mu \mathrm{m}$ ) beträgt, eine isotrope (Maxwellsche) Geschwindigkeitsverteilung und damit liegt thermisches Gleichgewicht vor [11 13]. In der Knudsenschicht ändern sich Temperatur, Druck und Dichte diskontinuierlich [157]; man kann sie folglich nicht als Kontinuum behandeln und benutzt deshalb Methoden wie zur Berechnung starker Schockwellen. Außerdem nimmt man für deren Absorptionseigenschaften die des Festkörpers an. Ein Simulations-Programm von Fähler und Krebs [158] berechnet mithilfe der Gl. 4.10 und (4.13) die Laser-Ablationsraten von Eisen im Vakuum (Parameter: $t_{p}=30 \mathrm{~ns}$ und $\lambda=248 \mathrm{~nm}$ für KrFExcimer). Weil ein beträchtlicher Anteil des verdampften Materials die Laserstrahlung bereits absorbiert hat, muss die Eindringtiefe noch um diesen Beitrag $z_{D}$, die in der Größenordnung der Absorptionslänge liegt, korrigiert werden:

$$
I(z)=A \cdot I_{0} \cdot \exp (-\alpha z) \cdot \exp \left(-\alpha z_{D}\right)
$$

\subsubsection{Abkühlrate und Temperaturgradient}

Der Temperaturgradient $\partial T / \partial z$ kann durch

$$
\frac{\partial T}{\partial z} \simeq \frac{\Delta T}{\sqrt{2 \kappa \tau_{p}}}
$$

abgeschätzt werden [159], wenn die Bedingung

$$
\sqrt{2 \kappa \tau_{p}}>\alpha^{-1}=l_{\alpha}
$$

erfüllt ist [160]. $\Delta T$ ist die Differenz von Oberflächentemperatur (Endtemperatur, z.B. $2000 \mathrm{~K}$ ) und Substrattemperatur (Raumtemperatur, $295 \mathrm{~K}$ ), $\kappa$ die Diffusivität und $\tau_{p}$ die Pulsdauer. Dieser Wert liegt im Bereich $\partial T / \partial z \simeq 2 \cdot 10^{7} \mathrm{~K} / \mathrm{cm}$. Der Wärmefluss $\Phi$ errechnet sich zu

$$
\Phi=\lambda \cdot\left(\frac{\partial T}{\partial z}\right)
$$

dabei ist $\lambda$ die spezifische Wärmeleitfähigkeit 13. Mit der thermischen Diffusionslänge $\ell_{D}=2 \sqrt{\kappa \tau_{p}}$, ergibt sich für die Abkühlrate

$$
\dot{T}=\frac{\lambda \cdot\left(\frac{\partial T}{\partial z}\right)}{c_{p} \rho \ell_{D}}=\frac{\kappa}{\ell_{D}} \cdot\left(\frac{\partial T}{\partial z}\right) \sim \frac{\Delta T}{\tau_{p}},
$$

mit der Dichte $\rho$ und der spezifischen Wärmekapazität $c_{p}$ des Materials [13]. Die Abkühlrate $\dot{T}$ liegt hier in der Größenordnung von einigen $10^{10} \mathrm{~K} / \mathrm{s}$. 


\subsection{Numerische Berechnung der Temperatur-Profile}

Bei numerischen Rechenmethoden werden die auftretenden Differenziale durch Differenzenquotienten ersetzt 161, 162]. Die Diskretisierung wird mittels geeigneter Differenzen $\Delta t$ und $\Delta z$ durchgeführt, die sich aus den thermischen Eigenschaften des Materials ergeben. Für Eisen sind die Werte $\Delta z \sim 10 \mathrm{~nm}$ und $\Delta t \sim 1$ ps. Die relevanten Größen $t$ und $z$ werden anschließend in konstante Intervalle $t^{i}=i \cdot \Delta t$ und $z_{n}=n \cdot \Delta z$ eingeteilt. Das Argument einer Funktion wird folgendermaßen abgeändert: $T(z, t) \rightarrow T\left(z_{n}, t^{i}\right)$. Die Rechenvorschrift für die 1. Ableitung nach der Zeit ist dann durch

$$
\frac{\partial T}{\partial t}=\frac{T^{i+1}-T^{i}}{\Delta t}
$$

gegeben. Für die 1. und 2. Ortsableitung gilt

$$
\frac{\partial T}{\partial z}=\frac{T_{n+1}-T_{n}}{\Delta z} \quad \text { bzw. } \quad \frac{\partial^{2} T}{\partial z^{2}}=\frac{T_{n-1}-2 T_{n}+T_{n+1}}{(\Delta z)^{2}}
$$

Zusammengefasst ergibt sich die Wärmeleitungsgleichung als sog. FTCS-Schema (forward time centred space). Der Name rührt daher, auf welche Weise die Differenzen gebildet werden [162]:

$$
T_{n}^{i+1}=T_{n}^{i}+\Delta t \cdot\left[\frac{\kappa}{(\Delta z)^{2}}\left(T_{n-1}^{i}-2 T_{n}^{i}+T_{n+1}^{i}+\frac{Q}{\rho c_{p}}\right)\right]
$$

Da weiterhin $\Delta x$ und $\Delta t$ nicht unabhängig voneinander sind, muss das Neumannsche Stabilitätskriterium

$$
\frac{\Delta t}{(\Delta z)^{2}} \geq \frac{1}{2 \kappa}
$$

erfüllt werden, damit eine physikalische Lösung erhalten werden kann. Die folgenden Abbildungen zeigen die errechneten Oberflächentemperaturen (Abb. 4.1) und die Aufschmelztiefe (Abb. 4.2) bei verschiedenen Intensitäten: Die Geschwindigkeit der Fest-Flüssig-Grenzschicht (Schmelzfront) $v_{l s}$ in der Nähe der

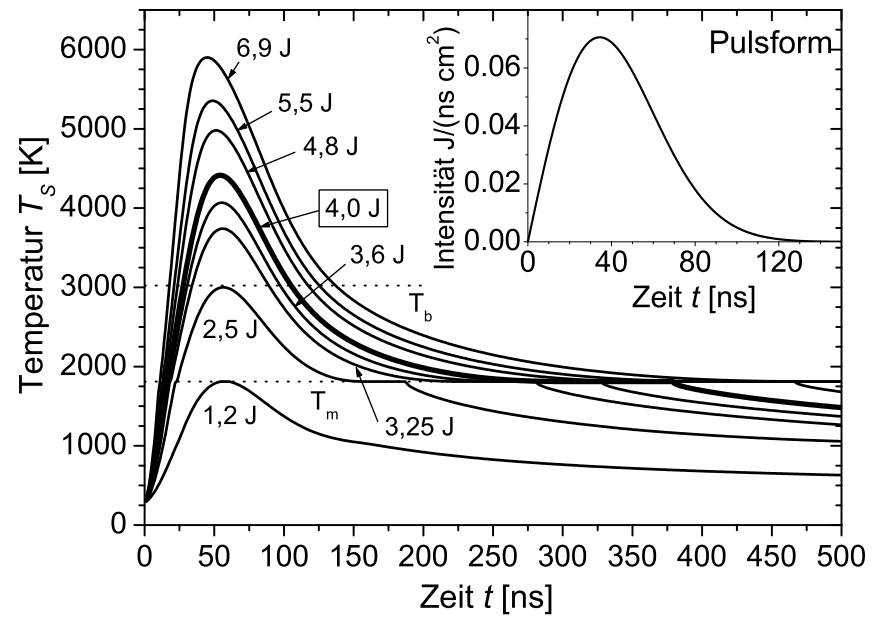

Abbildung 4.1: Mit dem Simulationsprogramm 24 errechnete zeitliche Entwicklung der Oberflächentemperatur von Eisen. Schmelz- $T_{m}$ und Siedetemperatur $T_{b}$ sind angegeben. Im oberen Teil ist die Form des Pulses dargestellt.

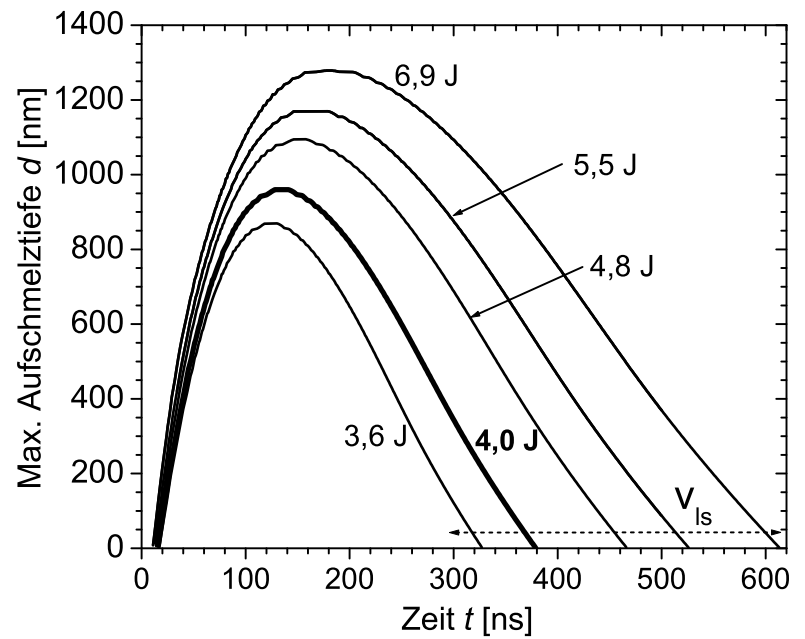

Abbildung 4.2: Zeitliche Entwicklung der Aufschmelztiefe von Eisen (errechnet nach [24]). Die Geschwindigkeit der Schmelzfront $v_{l s}$ beträgt ungefähr $4,0(4) \mathrm{m} / \mathrm{s}$. (Position durch Doppelpfeil gekennzeichnet).

Oberfläche errechnet sich aus der Steigung des rechten Astes der Schmelzkurve zu ca. 4,0(4) m/s.

\subsection{Materialverdampfung und Plasmabildung}

Die Intensität bestimmt im wesentlich die Prozesse, die direkt über der Materialoberfläche und dem umgebenden Medium stattfinden. Simulationsrechnung zeigen, dass die Materialverdampfung erst ab 
ca. $2,5 \mathrm{~J} / \mathrm{cm}^{2}$ auftritt 163 (s. Abb. 4.1). Bei niedrigen Intensitäten beschränkt sich die Laser-MetallWechselwirkung auf Prozesse wie Schmelzen und Verdampfen. Bei höheren Intensitäten wird der Metalldampf ionisiert, was zu einer weiteren Absorption führt. Der Prozess führt zu einem enormen Temperaturanstieg und die Bildung eines Plasmas setzt ein. Diese Plasmaschwelle konnte rechnerisch in guter Übereinstimmung mit dem Experiment ermittelt werden, in denen $\mathrm{Al}$ und $\mathrm{Fe}$ mit einem Excimerlaser (XeF, $\lambda=350 \mathrm{~nm}$ ) bestrahlt wurden [164 165]. Die Kollisionen zwischen den Atomen in der Gasphase und den Elektronen bestimmen den Ionisierungsgrad $n_{e} /\left(n_{e}+n_{a}\right)$ des Plasmas, der für jede Temperatur durch die Saha-Gleichung [166, 11] angegeben werden kann:

$$
\frac{\left(\frac{n_{e}}{n_{e}+n_{a}}\right)^{2}}{1-\left(\frac{n_{e}}{n_{e}+n_{a}}\right)}=\frac{2 g_{i}}{g_{a}\left(n_{e}+n_{a}\right)} \cdot\left(\frac{2 \pi m_{e} k_{B} T}{h^{2}}\right)^{3 / 2} \exp \left(-\frac{E_{I}}{k_{B} T}\right),
$$

dabei bedeuten $m_{e}$ : Elektronenmasse, $h$ : Planck-Konstante, $k_{B}$ : Boltzmann-Konstante und $T$ : Temperatur $[\mathrm{K}]$ und $E_{I}$ : Ionisierungsenergie. $n_{e}$ ist die Elektronen- und $n_{a}$ die Atom-/Moleküldichte. $g_{i}$ und $g_{a}$ bezeichnen die Niveau-Entartung eines Quantenzustandes der Ionen bzw. Atome/Moleküle (für die Drehimpulsquantenzahl $J$ gilt dann $g_{i, a}=2 J+1$ ). Die Gl. (4.24) gilt aber nur für die einfache Ionisation, d.h. $n_{e}=n_{i}$ mit der Dichte der Ionen $n_{i}$. Man kann die Saha-Gleichung auf jede Ionisierungsstufe und für mehrere Gase verallgemeinern, doch die komplexen Gleichungssysteme können dann nur noch numerisch gelöst werden [167]. Der dominierende Absorptionsmechanismus macht bei hoher Ionisation die sog. inverse Bremsstrahlung aus. Die Energieübertragung erfolgt dabei über Frei-Frei-Übergänge der Elektronen. Der Absorptionskoeffizient ist gegeben durch [11, 13]:

$$
\alpha_{\mathrm{IB}} \approx \mathcal{K} \cdot \lambda^{3} \cdot \frac{Z^{2} n_{i} n_{e}}{\sqrt{T}}\left[1-\exp \left(\frac{\hbar \omega}{k_{B} T}\right)\right] \quad, \quad[\lambda]=\mu m
$$

mit

$$
\mathcal{K} \approx \frac{2 \sqrt{2} \cdot e^{6}}{3 \sqrt{3 \pi k_{B} m_{e}^{3}} \hbar c^{4}} \quad, \quad \text { Zahlenwert von } \mathcal{K} \approx 1,37 \cdot 10^{-35}
$$

Ein weiterer Absorptionsprozess, der über hochangeregte Atome/Moleküle abläuft, ist die (Multi-)Photoionisation, bei der Gebunden-Frei-Übergänge eine Rolle spielen (ein Gebunden-Gebunden-Übergang wäre die Photo-Anregung). Die Verdampfung des Targetmaterials ist demnach notwenig für die Zündung eines Plasmas, was die typischen Intensitäten von $\left(10^{9}-10^{11} \mathrm{~W} / \mathrm{cm}^{2}\right)$, die nötig sind, um Gase in Abwesenheit eines Materials zu ionisieren, um mehrer Größenordnungen verringern kann [24].

Die Dynamik des Plasmas bzw. Laser-Plumes ist in vielen Modellrechnungen untersucht worden. Das Model von Singh und Narayan [168] beschreibt die Entwicklung des Laser-Plasmas in 2 Stufen:

a) Während des Laserpulses (FWHM) $\tau_{p}$ expandiert das Plasma isotherm. Dabei wird die ideale Gasgleichung $p \cdot V=n k_{b} \cdot T$ benutzt.

b) nach der Pulsterminierung erfolgt dann die Expansion adiabatisch gemäß $V \sim T^{\frac{1}{1-\gamma}}$. Das Modell gilt nur bei niedrigen Drücken. Bei hohen Drücken ist es jedoch nicht mehr anwendbar, da die ideale Gasgleichung dann nicht mehr gültig ist.

Das Modell von Arnold et al. [169], nach dem der Laser-Plume sphärisch expandiert, zieht Massen-, Energie- und Impulserhaltung heran. Dieses Modell gilt sowohl bei niedrigem als auch bei hohem Druck, beschreibt aber die Dynamik erst nach der Pulsterminierung.

\subsubsection{Laser-Supported Absorption-Waves}

Das heiße Plasma breitet sich mit hoher Geschwindigkeit in die umgebende Atmosphäre aus, an dessen Grenzfläche eine Schockwelle entsteht (Abb. 4.3). Das Gas wird einerseits durch die Kompression der Schockwelle und andererseits durch Wärmeleitung bzw. Strahlung des Plasmas erhitzt. Erreichen die freigesetzten thermischen Elektronen eine kritische Elektronendichte, absorbiert nun auch das komprimierte Gas die Laserstrahlung und es wird selbst zum stark absorbierenden Plasma, das als laserinduzierte Absorptionswelle oder LSAW (laser-supported absorption wave) bezeichnet wird. Die LSAW 


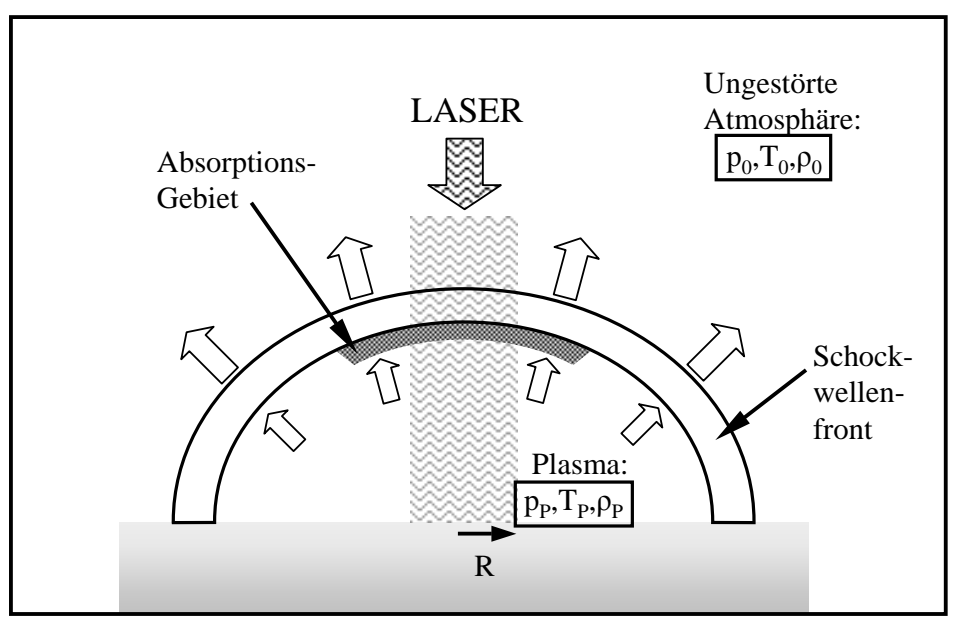

Abbildung 4.3: Radiale bzw. axiale Schockwellenausbreitung und thermodynamische Größen eines laserinduzierten Plasmas (frei nach [170]).

wird in 2 Klassen eingeteilt (z.B. [170]), die je nach Intensität $I_{0}$ des Lasers unterschiedliche Absorptionsund Ausbreitungs-Eigenschaften besitzen. Mitte der 1960er bis Anfang der 1970er Jahren wurden umfassende Theorien vor allem von Pirri und Raŭzer über die Dynamik und Entstehung der LSAWs entwickelt 171, 172, 173 174 175 176]. Eine tabellarische Zusammenfassung weiterer 1-dimensionaler Modelle und ihrer Ergebnisse ist in [157] zu finden.

1) Laser-supported Combustion Wave (LSCW): Die Plasmafront ist langsamer als die Schockwelle und bewegt sich mit Unterschallgeschwindigkeit. Beide Wellen sind räumlich getrennt und wandern auseinander. Ein Teil des Laser-Plumes wird ionisiert und absorbiert Laserenergie, die das entstehende Plasma weiter aufheizt. Das Plasma bleibt aber größtenteils transparent, so dass die Laserstrahlung noch das Target erreicht. Die Schockwelle komprimiert das umgebende Gas. Strahlung und Wärmeleitung des Plasmas sind hier maßgebend für die Aufheizung.

2) Laser-supported Detonation Wave (LSDW): Bei höheren Intensitäten wird das Plasma dichter und wegen erhöhter Absorption der Laserstrahlung, wird es stärker erhitzt. Die Plasmafront erreicht dabei Überschallgescheindigkeit und durch die enorme Kompression wird das Gas derart erhitzt, dass die komplette Strahlung in der Schockwellenfront absorbiert wird. Die Strahlung dringt deshalb nicht mehr zur Oberfläche, und die Temperatur des Plasmas ist im Vergleich zur LSC-Welle geringer; wegen der Abschirmung findet eine Laser-Materie-Entkopplung statt, so dass der Kontakt des Plasmas mit der Oberfläche abreißen kann.

Für die Laser-Material-Wechselwirkung ist es also vorteilhaft, im LSCW-Bereich zu arbeiten, weil die Energieeinkopplung aufgrund des Plasma-Kontaktes zur Oberfläche aufrecht erhalten bleibt. Für $4 \mathrm{~J} / \mathrm{cm}^{2}$ beträgt die Intensität des XeCl-Excimerlasers $7,27 \cdot 10^{7} \mathrm{~W} / \mathrm{cm}^{2}$ und liegt somit im LSCW-Bereich, weil $I_{0}>I_{\mathrm{LSCW}} \approx 10^{7} \mathrm{~W} / \mathrm{cm}^{2}$ und $I_{0}<I_{\mathrm{LSDW}} \approx 10^{8} \mathrm{~W} / \mathrm{cm}^{2}$ [177]. Im folgenden werden LSCW und LSDW der Reihe nach diskutiert.

Der Druck der LSC-Welle auf die Oberfläche beträgt nach [172, 170]:

$$
p_{\mathrm{LSCW}}=\left(1-\frac{2 \mathcal{W}}{\gamma_{0}-1}\right) \cdot\left(\frac{\gamma_{0}+1}{2}\right)^{1 / 3}\left(\frac{(\gamma-1)\left(\gamma_{0}+1\right)}{(\gamma+\mathcal{W})\left(\gamma_{0}-1-2 \mathcal{W}\right)}\right)^{2 / 3} \rho_{0}^{1 / 3} \cdot I_{0}^{2 / 3} \propto I_{0}^{2 / 3}
$$

dabei sind: $\gamma \simeq 1,2$ der Adiabatenexponent des Plasmas/Metalldampfes [178, 163], $\gamma_{0}=1,3053$ der Adiabatenexponent [179] und $\rho_{0}=0,671 \mathrm{~kg} / \mathrm{m}^{3}$ Dichte [180] der Methan-Atmosphäre. Die Ausbreitungsgeschwindigkeit $v_{\mathrm{LSCW}}$ der Welle ist

$$
v_{\mathrm{LSCW}}=(1+\mathcal{W})\left(\frac{2(\gamma-1)\left(\gamma_{0}-1\right)}{\left(\gamma_{0}+1\right)(\gamma+\mathcal{W})\left(\gamma_{0}+1-2 \mathcal{W}\right)} \cdot \frac{I_{0}}{\rho_{0}}\right)^{1 / 3} \propto I_{0}^{1 / 3}
$$

$\mathcal{W}$ ist die dimensionslose Ausbreitunggeschwindigkeit und kann angenähert werden durch 170

$$
\mathcal{W} \approx 0,009 \cdot I_{0}^{2 / 3} \quad, \quad\left[I_{0}\right]=\mathrm{MW} / \mathrm{cm}^{2}
$$


Der große Anteil der Laserenergie erhitzt das Plasma und dieses wiederum die umgebende Atmosphäre aufgrund Wärmeleitung und -strahlung. Der verbleibende Teil der Energie wird in kinetische Energie des Plasma-Plumes konvertiert. Betrachtet man den Energieverlust pro Volumen $E_{\text {verl }}\left[\mathrm{J} / \mathrm{cm}^{3}\right] \mathrm{im}$ Plasma, dann beträgt die Energie-Bilanz [175, 13]:

$$
\frac{\lambda_{\mathrm{eff}} \cdot \Delta T}{d}=d \cdot\left(\alpha I_{0}-E_{\mathrm{verl}}\right)
$$

dabei ist: $d$ die Dicke der (als Scheibe betrachteten) LSCW-Front, $\Delta T$ der Temperatursprung entlang der LSC-Welle, $\alpha$ Absorptionskoeffizient des Plasmas und $\lambda_{\text {eff }}=\lambda_{\text {diff }}+\lambda_{\text {str }}$ die thermische Leitfähigkeit, die sich aus einem diffusiven und einem Strahlungsanteil [178 zusammensetzt. Benutzt man die Wärmeleitungsgleichung (4.10), so erhält man:

$$
\left(\rho c_{p} \cdot \frac{\partial T}{\partial t}=\rho c_{p} \frac{\Delta T}{\Delta z} v_{\mathrm{LSCW}}=\right) \quad \rho c_{p} \frac{\Delta T}{d} v_{\mathrm{LSCW}} \stackrel{!}{=} \lambda_{\mathrm{eff}} \frac{\Delta T}{d^{2}} \quad\left(=\lambda_{\mathrm{eff}} \frac{\partial^{2} T}{\partial z^{2}}\right)
$$

Einsetzen von Gl. 4.30 ergibt 13

$$
v_{\mathrm{LSCW}}=\frac{\lambda_{\mathrm{eff}}}{\rho c_{p}} \sqrt{\frac{\alpha I_{0}-E_{\mathrm{verl}}}{\lambda_{\mathrm{eff}} \cdot \Delta T}}
$$

Ist die Intensität so gewählt, dass sie gerade die Verluste kompensiert $\alpha I_{0} \approx E_{\text {verl }}$, so bildet sich eine stationäre LSC-Welle mit $v_{\mathrm{LSCW}} \approx 0$ aus, ein sog. Plasmatron [181].

Bei höheren Intensitäten wird die Laserstrahlung komplett in der Schockwelle absorbiert. Der Druck in der entstehenden LSD-Welle ist dann:

$$
p_{\text {LSDW }}=\frac{\left(2\left(\gamma^{2}-1\right)\right)^{2 / 3}}{\gamma+1}\left(\frac{\gamma+1}{2 \gamma}\right)^{\frac{2 \gamma}{\gamma-1}} \rho_{0}^{1 / 3} I_{0}^{2 / 3} \propto I_{0}^{2 / 3}
$$

mit der Geschwindigkeit der LSD-Welle

$$
v_{\mathrm{LSDW}}=\left(2\left(\gamma^{2}-1\right) \frac{I_{0}}{\rho_{0}}\right)^{1 / 3} \propto \sqrt{\frac{p_{\mathrm{LSDW}}}{\rho_{0}}} \propto I_{0}^{1 / 3}
$$

Die bisher aufgeführten Modelle bzw. Gleichungen beinhalten nur eine 1-dimensionale, axiale Ausbreitung des Plasmas. Reilly et al. [182] betrachtet darüber hinaus auch die radiale Expansion des Plasmas bis zum Rand des Laserpulses und führt ein 2-dimensionales Modell ein, das auch die zeitliche Dynamik beschreibt und dem realen Plasma besser angepasst ist. Aufgrund der Expansion ensteht hinter der Plasmafront eine Zone mit vermindertem Druck, so dass die radiale Verdünnungswelle mit der lokalen Schallgeschwindigkeit $c_{S}$ in der Zeit $\tau_{2 D}$ vom Rand nach innen läuft.

$$
\tau_{2 D}=\frac{r_{P}}{c_{S}}
$$

$r_{P}$ ist der Radius der Laserspots. Die Schallgeschwindigkeit im LSCW-Bereich errechnet sich zu:

$$
c_{S}=\left(\frac{\gamma \cdot p_{\mathrm{LSCW}}}{\rho_{0}}\right)^{1 / 2}\left(\frac{(\mathcal{W}+1)\left(\gamma_{0}-1\right)}{\mathcal{W}\left(\gamma_{0}+1\right)}\right)^{1 / 2}
$$

Die zweite, axiale Verdünnungswelle beginnt von der Plasmafront zur Oberfläche zurückzulaufen, wenn der Laserpuls nach der Zeit $\tau_{P}$ terminiert und die Energieeinkopplung in das Plasma stoppt. Das Plasma dehnt sich noch weiter aus, kühlt sich aber dabei ab. Die Verdünnungswelle erreicht die Oberfläche nach der Zeit

$$
\tau_{z}=\tau_{p}+\frac{v_{\mathrm{LSCW}} \cdot \tau_{p}}{c_{S}}
$$

Für die Intensität $I_{0} \simeq 6,55 \cdot 10^{7} \mathrm{~W} / \mathrm{cm}^{2}\left(3,6 \mathrm{~J} / \mathrm{cm}^{2}\right)$ und $r_{P}=2,82 \mathrm{~mm}$ erhält man beispielsweise: $\tau_{z}=226 \mathrm{~ns}$ und $\tau_{2 D}=449 \mathrm{~ns}$. Die zeitliche Entwicklung des Oberflächendruckes im Zentrum des Laser- 


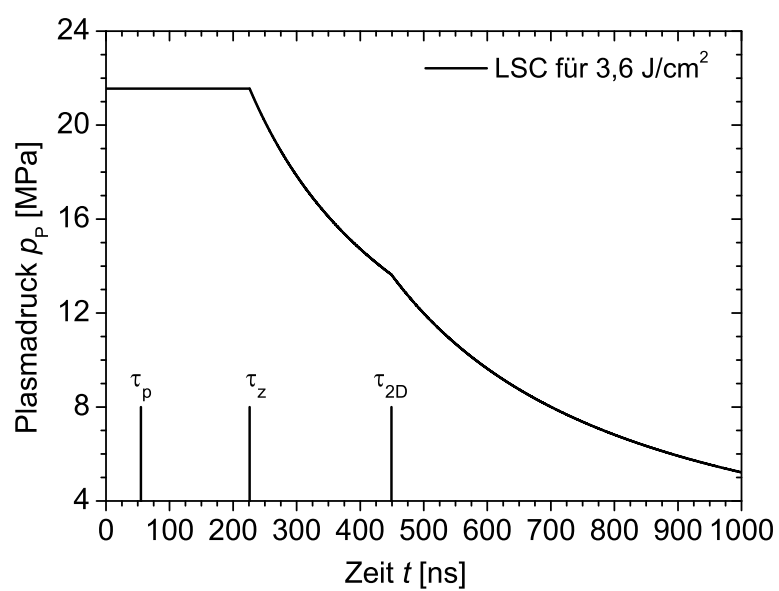

Abbildung 4.4: Die zeitliche Entwicklung des LSCWPlasmadruckes auf der Oberfläche bei $I_{0} \simeq 6,55 \cdot 10^{7}$ $\mathrm{W} / \mathrm{cm}^{2}$.

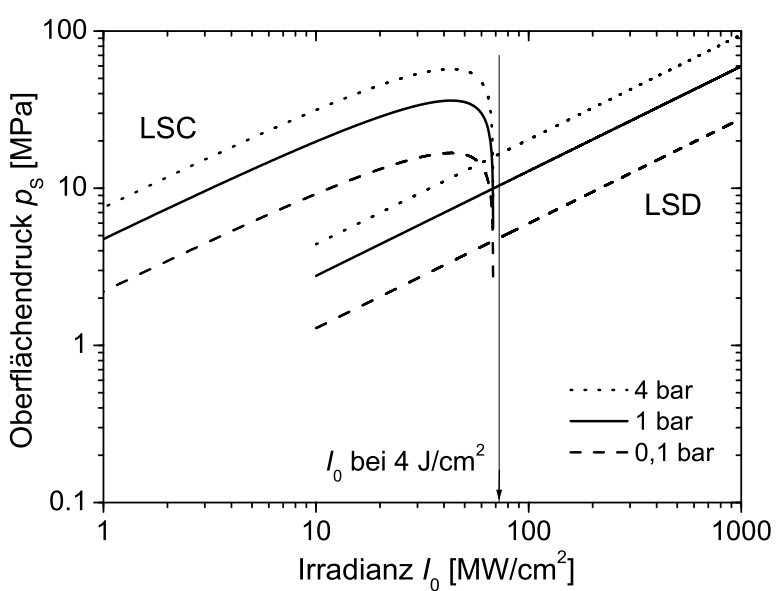

Abbildung 4.5: Abhängigkeit des Oberflächendruckes von der Intensität für LSC- und LSD-Wellen.

spots kann für den LSCW-Bereich mit den charakteristischen Zeiten $\tau_{z}$ und $\tau_{2 D}$ folgendermaßen geschrieben werden [182]:

$$
\begin{aligned}
t \leq \tau_{z} \quad: \quad p_{P}(t)=p_{\mathrm{LSCW}} \\
\tau_{z} \leq t \leq \tau_{2 D} \quad: \quad p_{P}(t)=p_{\mathrm{LSCW}} \cdot\left(\frac{t}{\tau_{z}}\right)^{-2 / 3} \\
\tau_{2 D} \leq t \quad: \quad p_{P}(t)=p_{\mathrm{LSCW}}\left(t=\tau_{2 D}\right) \cdot\left(\frac{t}{\tau_{2 D}}\right)^{-6 / 5}
\end{aligned}
$$

Aus Abb. 4.5 ist ersichtlich, dass das LSCW-Modell im Bereich der üblichen Intensität von $I_{0} \simeq 7,27 \cdot 10^{7}$ $\mathrm{W} / \mathrm{cm}^{2}\left(4 \mathrm{~J} / \mathrm{cm}^{2}\right)$ gerade nicht mehr gültig ist. Root 170 gibt als unterste Grenze des LSDW-Bereichs $10^{7} \mathrm{MW} / \mathrm{cm}^{2}$ an, während Herziger und Kreutz für KrF-Bestrahlung ( $\left.\lambda=248 \mathrm{~nm}, \mathrm{FWHM}=50 \mathrm{~ns}\right)$ von Stahl $(2-4) \cdot 10^{8} \mathrm{~W} / \mathrm{cm}^{2}$ angeben [146, 183], was mit den Bedingungen dieses Lasers und Materials fast übereinstimmt; daher ist diese Untergrenze hier auch anzunehmen. Da man sozusagen zwischen LSCWund LSDW-Bereich operiert, ist es schwierig, ein bestimmtes Modell für weitere Rechnungen in Betracht zu ziehen.

\subsection{Materietransport}

Bei der Laserbestrahlung wird nicht nur Materie verdampft und senkrecht von der Oberfläche weggeschleudert, sondern es findet auch lateraler Materietransport statt, der durch Temperaturgradieneten in der Schmelze verursacht wird : Beim thermo-kapillaren Effekt verursacht die temperaturabhängige Oberflächenspannung $d \sigma / d T$ eine Materiebewegung, deren laterale Geschwindigkeitskomponente durch

$$
v_{\mathrm{tc}}=\frac{\delta_{m} \cdot \Delta T}{\eta \cdot d_{L}} \cdot \frac{d \sigma}{d T}
$$

abgeschätzt werden kann (sog. Marangoni-Konvektion) [11]. Dabei bedeuten: dynamische Viskosität $\eta=6,9 \cdot 10^{-3} \mathrm{~Pa} \cdot \mathrm{s}$ [184], Temperatur-Koeffizient der Oberflächenspannung $d \sigma / d T=-5 \cdot 10^{-4} \mathrm{~N} /(\mathrm{mK})$ [185], $\delta_{m} \approx 1 \mu \mathrm{m}$ Dicke der geschmolzenen Schicht, $d_{L}$ Durchmesser des Laserspots. Der laterale Temperaturunterschied kann mit $\Delta T \approx 4700 \mathrm{~K}$ angegeben werden [144]. Für einen oberen Grenzwert ergibt sich $v_{\text {tc }} \approx 0,2 \mathrm{~m} / \mathrm{s}$. Der weitaus überwiegendere Transport-Mechanismus ist der sog. Piston-Effekt. Der auf die Oberfläche ausgeübte Plasmadruck $p_{\mathrm{Pl}}$ und Rückstoßdruck $p_{\text {rec }}$ des verdampfenden Metalls ist radial inhomogen, weil die Energiedichte ebenfalls radial variiert. Dadurch entstehen Druckgradienten, die das Material seitlich mit der Geschwindigkeit $v_{m}$ herausschleudern, so als ob ein „Kolben“ senkrecht auf die Schmelze der Dicke $\delta_{m}$ auftreffen würde und den Flüssigkeitsspiegel so herunterdrückt [186, 187]. 


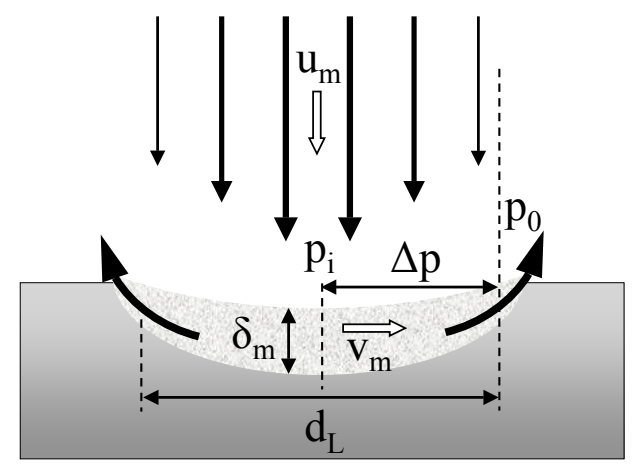

Abbildung 4.6: Piston-Effekt: Die Druckdifferenz verursacht eine Materiebewegung und schleudert sie radial nach außen.

Die Abb. 4.6 zeigt das Prinzip diese Effektes: Nach 13, 187 kann die Geschwindigkeit aus der Gleichheit der Volumenarbeit und kinetischen Energie abgeschätzt werden. Dazu nimmt man die Schmelze als nicht-viskos und inkompressibel an:

$$
v_{\mathrm{P}}=\sqrt{\frac{2 \Delta p}{\rho}}=\sqrt{\frac{2\left(\left(p_{\mathrm{Pl}}+p_{\mathrm{rec}}\right)-p_{0}\right)}{\rho}}
$$

$\Delta p$ ist die Differenz zwischen der Summe $p_{i}$ aus Plasmadruck $p_{\mathrm{Pl}}$ und Rückstoßdruck $p_{\text {rec }}$ und dem Umgebungsdruck $p_{0}$. Für $\Delta p=100$ bar [13] und der Dichte des flüssigen Eisens $\rho \approx 7 \mathrm{~g} / \mathrm{cm}^{3}$ [188] ergibt sich eine radiale Geschwindigkeit von ca. $50 \mathrm{~m} / \mathrm{s}$. In [131] wurden detaillierte Berechnungen angestellt und für eine Energiedichte $H=4 \mathrm{~J} / \mathrm{cm}^{2}$ und die daraus resultierende Druckdifferenz von $\Delta p \approx 7,8 \cdot 10^{2}$ bar ein Wert von $v_{P} \approx 148 \mathrm{~m} / \mathrm{s}$ erhalten. Man kann nun die Geschwindigkeit $u_{m}$ berechnen, mit der sich der Kolben herunterbewegt. Wegen vorausgesetzter Inkompressibilität ist der durch den Kolben hervorgerufene Materiefluss $j_{P}$ gleich dem radial nach außen strömenden Fluss $j_{r}$ :

$$
j_{P}=j_{r} \quad \Leftrightarrow \quad \rho u_{m} \cdot A=l \delta_{m} \rho v_{m}
$$

$A$ ist die Fläche des Laserspots, $l=\pi d_{L}$ dessen Umfang und $\kappa$ die Diffusivität. Die Tiefe der Schmelze läßt sich mit

$$
\delta_{m}=\frac{\kappa}{v_{m}} \cdot \ln \left(\frac{T_{s d}}{T_{s m}}\right)
$$

berechnen. Daraus ergibt sich schließlich:

$$
u_{m}=\sqrt{\frac{l \kappa \cdot \ln \left(T_{s d} / T_{s m}\right)}{A}} \cdot\left(\frac{2 \Delta p}{\rho}\right)^{1 / 4}
$$

Mithilfe von Simulationsrechnungen kann man $\delta_{m}$ direkt bestimmen, was sich besser zur Berechnung des Piston-Effektes eignet [131]. Die Absenkung der Oberfläche $\Delta z_{p}$ kann dann durch Integration von Gl. 4.43 bestimmt werden

$$
\Delta z_{p}=\int_{0}^{t} \frac{l \cdot \delta_{m}^{c a l c}}{A} \cdot \sqrt{\frac{2 \Delta p}{\rho}} d t
$$

was für $H=4 \mathrm{~J} / \mathrm{cm}^{2}, \Delta p \approx 7,8 \cdot 10^{2}$ und einer Fläche von $2 \times 3 \mathrm{~mm}^{2}$ in der Größenordnung von $\Delta z_{p}=12(4) \mathrm{nm} /$ Puls liegt.

Die Konvektion oder Wirbelbildung, die durch oben genannte Effekte verursacht wird, ermöglicht einen homogenen Materietransport in Tiefen bis $10 \mu \mathrm{m}$ (Carburisieren von Ti mit einem Excimer-Laser [26] bzw. von Fe mit einem $\mathrm{CO}_{2}$-Laser [25]). Die Dicke beträgt also ca. das 10-fache der Aufschmelztiefe und kann auch durch Kohlenstoff-Diffusion in flüssiger Phase nicht erreicht werden. 



\section{Kapitel 5}

\section{Ergebnisse der Eisen-Bestrahlung}

\subsection{Druckserie}

Als erstes werden die Druck- und Pulsserien diskutiert. Die Abb. 5.1 zeigt die RBS Roh-Spektren der Druck-Serie bei $11 \times 12$ Pulsen; der Deutlichkeit halber sind nur die Extrema bei $\mathrm{p}\left(\mathrm{CH}_{4}\right)=0,1$ und 10 bar Methan abgebildet. Die Probe bei $\mathrm{p}\left(\mathrm{CH}_{4}\right)=0,1$ bar Methan-Druck weist an der Basis der Eisenkante einen Knick auf, der Kohlenstoff auf der Oberfläche zugeordnet werden kann. Der Kohlenstoffanteil beträgt bis zu 100\%. Der Bulk-Anteil ist jedoch identisch mit dem der $\mathrm{p}\left(\mathrm{CH}_{4}\right)=10$ bar-Probe. Dieser Kohlenstoff liegt

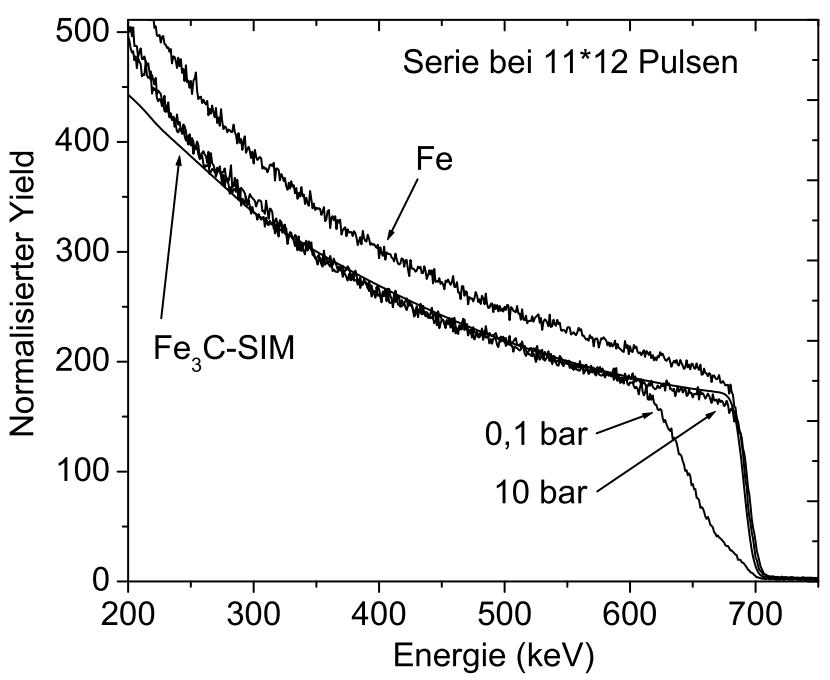

Abbildung 5.1: RBS-Spektren der Druck-Serie bei $11 \times 12$ Pulsen. Es sind lediglich die Extremaldrücke bei $\mathrm{p}\left(\mathrm{CH}_{4}\right)=0,1$ bar und 10 bar skizziert. $\mathrm{Fe}_{3} \mathrm{C}$ - SIM ist das simulierte RUMP-Spektrum.

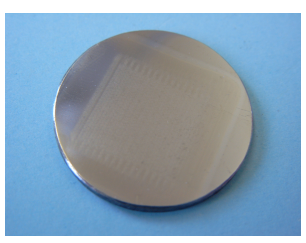

Abbildung 5.2: Probe bestrahlt bei $\mathrm{p}\left(\mathrm{CH}_{4}\right)=10$ bar und $11 \times 12$ Pulsen.

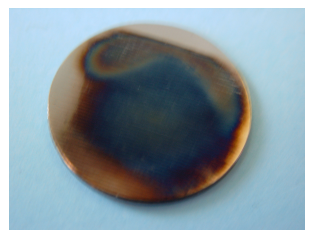

Abbildung 5.3: Probe bestrahlt bei $\mathrm{p}\left(\mathrm{CH}_{4}\right)=0,1$ bar und $11 \times 12$ Pulsen. Die Schwärzung stammt vom Kohlenstoff in der Oberfläche.

amorph vor, da die XRD-Spektren keinen Hinweis auf eine geordnete Struktur liefern. Raman-Messungen (s. Abschnitt 5.8) bestätigen die graphitische Struktur, wohingegen die Bildung von diamond-like carbon ausgeschlossen werden kann. Es entsteht während der Laserbestrahlung ein Kohlenstoff-Fallout, der sich nicht nur auf der Probe, sondern in der gesamten Kammer absetzt. Diese Schicht ist nicht homogen (s. Abb. 5.3). RBS-Messungen, die an verschiedenen Positionen auf der Oberfläche durchgeführt wurden, deuten auf unterschiedliche Dicken der Kohlenstoff-Schichten hin, jedoch ist der Kohlenstoff-Anteil im Bulk bei allen identisch. Bei hohem Druck hingegen bildet sich kein Kohlenstoff auf der Oberfläche (s. Abb. 5.2). Beide Aspekte werden für die weitere Diskussionen noch von Bedeutung sein. Ein weiterer wichtiger, entscheidender Punkt ist, über welchen Prozess der Kohlenstoff entsteht:

a) Das Methan dissoziiert im heißen Gas-Plasma (Entstehung im Kammervolumen).

b) Das Methan dissoziiert erst beim Kontakt mit dem Metalldampf (Entstehung im Kammervolumen).

c) Der Kohlenstoff entsteht aufgrund der hohen Temperaturentwicklung direkt an der Oberfläche. 
Mit dem Programm RUMP [189] wurde simuliert, dass bei beiden Extremaldrücken die gebildeten Fe-CVerbindungen ca. 25 at.\% Kohlenstoff enthalten. Diese Phasen besitzen aber unterschiedliche Strukturen (s. Kap. 2.1). XRD- und Mössbauermessungen (Abb.5.5 und 5.6 zeigen, dass es sich bei der HochdruckPhase um $\theta-\mathrm{Fe}_{3} \mathrm{C}$ und bei der Niederdruckphase um $\varepsilon$-Fe ${ }_{3} \mathrm{C}$ handelt. Das mit WiNDF [190] erstellte Kohlenstoffprofil in Abb. 5.4 zeigt ebenfalls eine hohe Kohlenstoffkonzentration auf der Oberfläche bei niedrigen Drücken. Im mittleren Bereich von ca. 70 bis $300 \mathrm{~nm}$ beträgt der Kohlenstoffanteil für alle Proben ungefähr 25 at.\%, der zu größeren Tiefen abfällt. Die Gleichskalierung der beiden Abzissen $\left(10^{15}\right.$ at. $/ \mathrm{cm}^{2}$

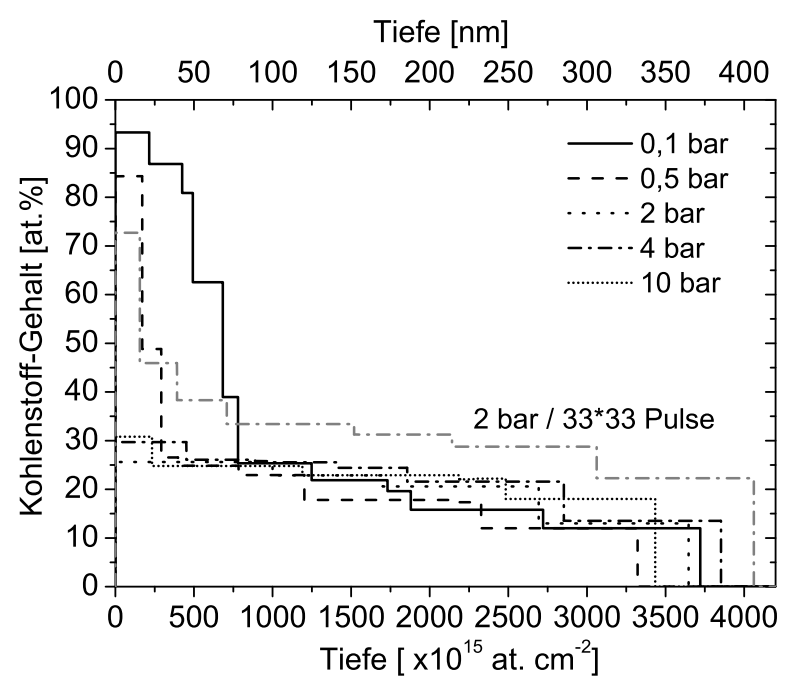

Abbildung 5.4: Kohlenstoffprofil der Druckserie. Zusätzlich ist noch eine $\mathrm{p}\left(\mathrm{CH}_{4}\right)=2$ bar-Probe bei $33 \times 33$ Pulsen dargestellt.

und nm) mit dem Faktor 10 ist eher zufällig. Die Umrechnung von $10^{15} \mathrm{at} . / \mathrm{cm}^{2}$ in die nm-Skala geschieht mittels des Faktors $f$ [86]:

$$
10^{15} \text { at. } / \mathrm{cm}^{2} \stackrel{\times \mathrm{f}}{\longrightarrow} \mathrm{nm}: \quad \mathrm{f}=\frac{1,661 \cdot 10^{-2} \cdot \mathrm{M}[\mathrm{amu}]}{\bar{\rho}\left[\mathrm{g} / \mathrm{cm}^{-3}\right]}
$$

errechnet und $\bar{\rho}$ die mittlere Dichte der Fe-C-Verbindung. Für die mittlere Molmasse eines $\mathrm{Fe}_{3} \mathrm{C}$-Teilchens $M\left(\mathrm{Fe}_{3} \mathrm{C}\right)=44,888 \mathrm{~g} / \mathrm{mol}$ und $\bar{\rho} \approx 7,6 \mathrm{~g} / \mathrm{cm}^{-3}$ ergibt sich gerade $f \simeq 0,098(1 / f \simeq 10,193)$. Geht man in der Berechnung der Tiefenskala von der Fe-C-Verbindung zu Kohlenstoff über, so ändert sich $f$ in einem annehmbaren Maße, und zwar derart, dass $[M / \bar{\rho}]_{C} \approx 6$ und $[M / \bar{\rho}]_{\mathrm{Fe}_{3} \mathrm{C}} \approx 5,72-6,67$, wenn man für die Dichte des Kohlenstoffs 1,8-2,1 $\mathrm{g} / \mathrm{cm}^{-3}$ annimmt [35].

Mithilfe der XRD-Reflexe kann die Entstehung und das Abnehmen der unterschiedlichen Carbid-Anteile gut verfolgt werden. Das XRD-Spektrum (Abb. 5.5) zeigt die verschiedenen Phasen bei gegebenem Druck. Nicht indizierte Peaks sind $\alpha$ - und $\gamma$-Fe. Der Übergang von $\varepsilon$-Carbid bei niedrigem Druck zu $\theta$-Carbid bei hohem Druck ist eindeutig erkennbar. Bei mittlerem Druck von ca. 2 bar liegt eine Mischung beider Phasen vor. Die vertikalen Linien geben die (ungefähre) Position der Carbide an. Unglücklicherweise sind einige Reflexlagen ähnlich, die zu verschieden Phasen gehören, besonders im hohen 2 $\theta$-Bereich. Für die Unterscheidung beider Phasen ist es daher wichtig, die Reflex-Lagen der Carbide bei den extremalen Drücken zu kennen (s. Kap. 5.2 Referenz-Proben). Betrachtet man beispielsweise die Peakpositionen ab ca. $2 \theta \approx 70^{\circ}$, so ist eine eindeutige Unterscheidung unmöglich, aber aufgrund der charakteristischen Reflexe der Phasen im niedrigen $2 \theta$-Bereich bis ca. $2 \theta \approx 55^{\circ}$ ist die $\varepsilon$ - von der $\theta$-Phase zu trennen.

Auch in den Mössbauerspektren kann man für beide Phasen typische Spektren beobachten und zur Identifizierung heranziehen. Die gemessenen Mössbauerspektren sind in Abb. 5.6 zusammengefasst. Die Niederdruck-Spektren zeigen überwiegend breite Spektren, wohingegen die Hochdruck-Phasen aus schmalen Spektren bestehen: Die $\varepsilon$-Phase ist durch maximal 3 breite, aufösbare Unterspektren charakterisiert. Die $\theta$-Phase wird durch 2 sehr eng benachbarte, schmale Unterspektren beschrieben. Das $\gamma-\operatorname{Fe}(\mathrm{C})$ wird durch ein Singulett und ein Doublett angefittet. Das Singulett weist eine Isomerieverschiebung von $I S \simeq-0,07(2) \mathrm{mm} / \mathrm{s}$ auf und ist Fe-Atomen zuzuschreiben, die keine nächsten C-Nachbarn besitzen. Das Doublett gehört zu einem Fe-Platz mit einem nächsten C-Nachbarn und zeigt eine relative starke 


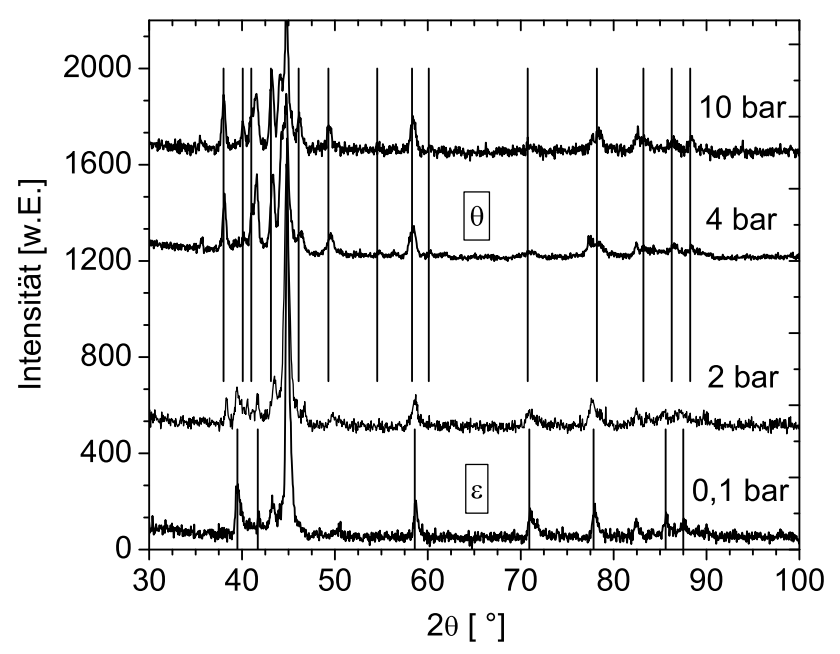

Abbildung 5.5: XRD-Spektrum der bei $11 \times 12$ Pulsen bestrahlten Proben unter verschiedenen Methan-Drücken. Die senkrechten Linien geben die Positionen der charakeristischen $\theta$ - und $\varepsilon$-Carbid-Peaks an.

Quadrupolwechselwirkung von $Q S \simeq 0,63(2) \mathrm{mm} / \mathrm{s}$; die Isomerieverschiebung beträgt $I S \simeq 0,045 \mathrm{~mm} / \mathrm{s}$ (z.B. [30]). Jedes Unterspektrum repräsentiert eine Phase (bzw. Teil einer Phase), der man einen relativen Flächenanteil zum Gesamtspektrum zuorden kann. Der Gesamtflächenanteil setzt sich dann additiv aus den Flächen der Unterspektren zusammen (Abb. 5.7): Da der Kohlenstoffgehalt einer Phase bekannt bzw.

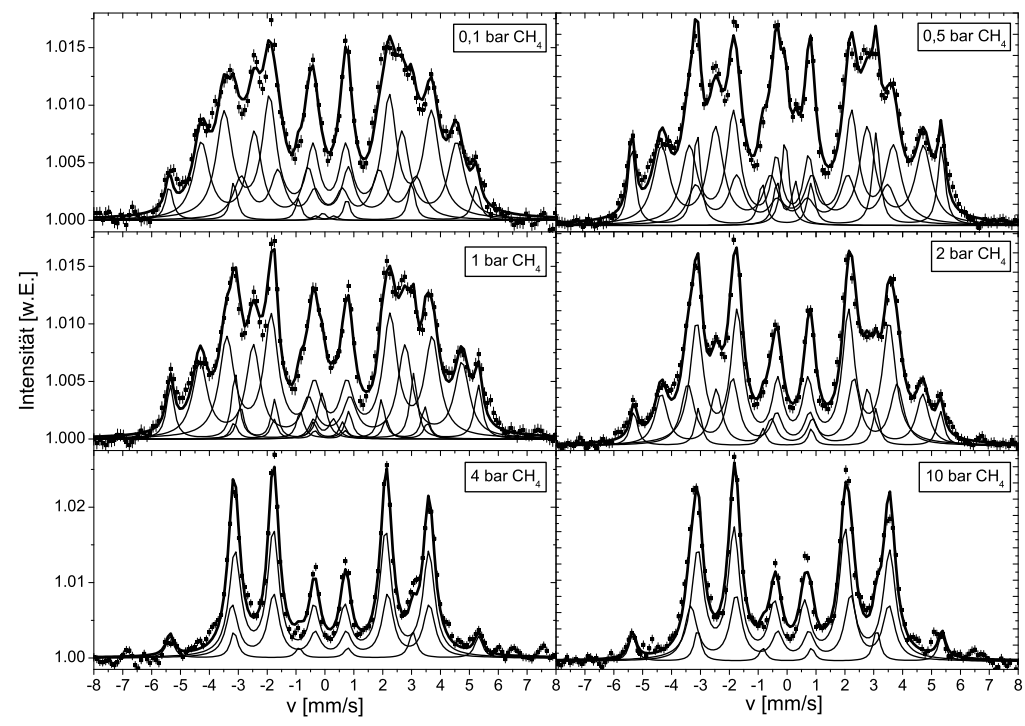

Abbildung 5.6: Mössbauerspektren der Druckserie bei $11 \times 12$ Pulsen

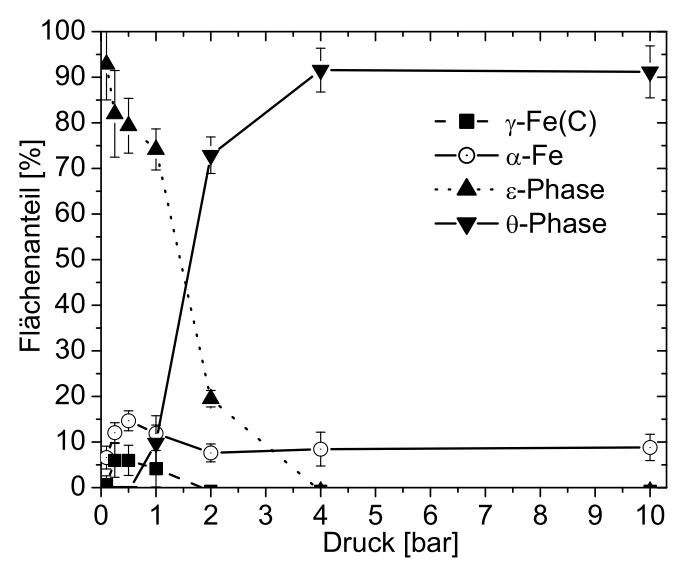

Abbildung 5.7: Flächenanteil der verschiedenen Phasen in der Druckserie bei $11 \times 12$ Pulsen.

errechnet werden kann, hat man mit der Mössbauerspektroskopie ein geeignetes Mittel an der Hand, den Kohlenstoffgehalt der Probe zu bestimmen.

Die folgenden Proben der Pulsserien wurden bei Drücken von 0,1, 1 und 4 bar Methan bestrahlt. Die Analyse wird analog der Druckserie durchgeführt.

\subsection{Referenz-Proben}

Bei den Bestrahlungen der Proben mit $11 \times 12$ Pulsen hat sich herausgestellt, dass sich bei einem Druck von $p\left(\mathrm{CH}_{4}\right)=0,1$ bar ausschließlich das $\varepsilon$-Carbid und bei $p\left(\mathrm{CH}_{4}\right)=4$ bar das $\theta$-Carbid bildet. Die Identifizierung der einzelnen Phasen wird mit der Powder-Diffraction Datenbank vorgenommen [34]. Die Gitterparameter der Referenz-Proben sind in Tab. 5.1 aufgelistet Im XRD-Spektrum ist das $\varepsilon$-Carbid als einzige Komponente sowohl in $750 \mathrm{~nm}\left(5^{\circ}\right.$ g.i.) und $150 \mathrm{~nm}\left(1^{\circ}\right.$ g.i.) erkennbar. Eine Informationstiefe von $150 \mathrm{~nm}$ liefert 


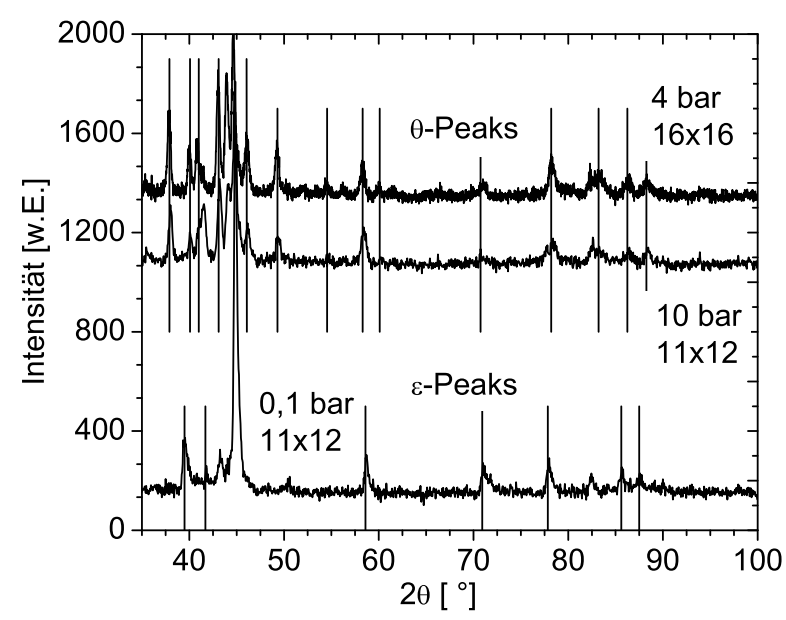

Abbildung 5.8: XRD-Spektren ( $5^{\circ}$ g.i.) der Proben, die ausschließlich nur $\varepsilon$ - oder $\theta$-Carbid enthalten und zur Indentifizierung dieser Phasen dienen.

Tabelle 5.1: Gitterparameter der Phasen der Referenz-Proben.

\begin{tabular}{|c||c||c|c|}
\hline $\begin{array}{c}\text { Gitter- } \\
\text { Parameter }[\AA]\end{array}$ & $\begin{array}{c}\varepsilon: 0,1 \mathrm{bar} / \\
11 \times 12\end{array}$ & $\begin{array}{c}\theta: 4 \mathrm{bar} / \\
16 \times 16\end{array}$ & $\begin{array}{c}\theta: 10 \mathrm{bar} / \\
11 \times 12\end{array}$ \\
\hline \hline$a$ & $2,66216(215)$ & $5,06741(627)$ & $5,06981(2053)$ \\
\hline$b$ & - & $6,75112(1283)$ & $6,71687(2986)$ \\
\hline$c$ & $4,35305(465)$ & $4,50209(664)$ & $4,50819(2258)$ \\
\hline
\end{tabular}

auch das CEM-Spektrum. Das breite Spektrum, wie es Abb. 5.9 zeigt, kann somit eindeutig dem $\varepsilon$-Carbid zugeordnet werden, das aus 3 gut auflösbaren Unterspektren besteht: Die Richtwerte der Hyperfeinfelder

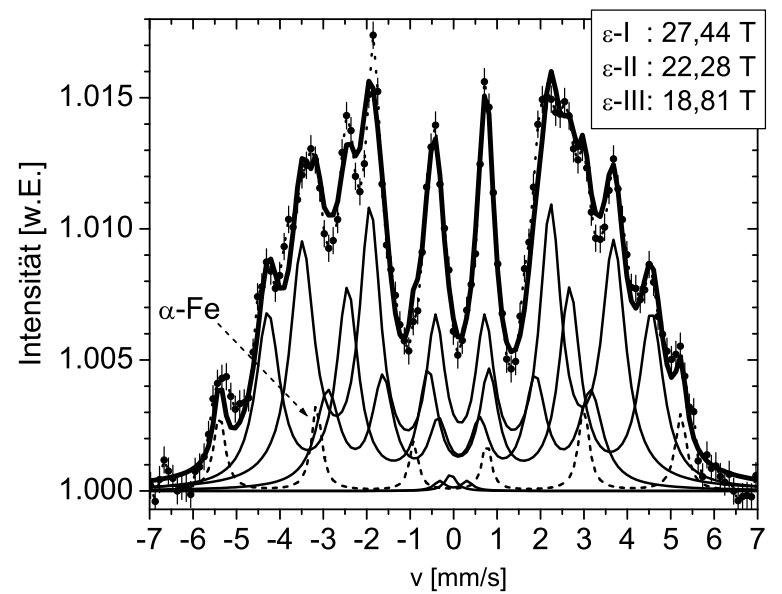

Abbildung 5.9: Gemessenes (gepunktet) und angefittetes (dicke Linie) Spektrum, das aus 3 Unterspektren mit den angegeben Feldern besteht. Die sehr schwachen Unterspektren in der Mitte sind dem $\gamma$-Fe(C) zuzuordnen.

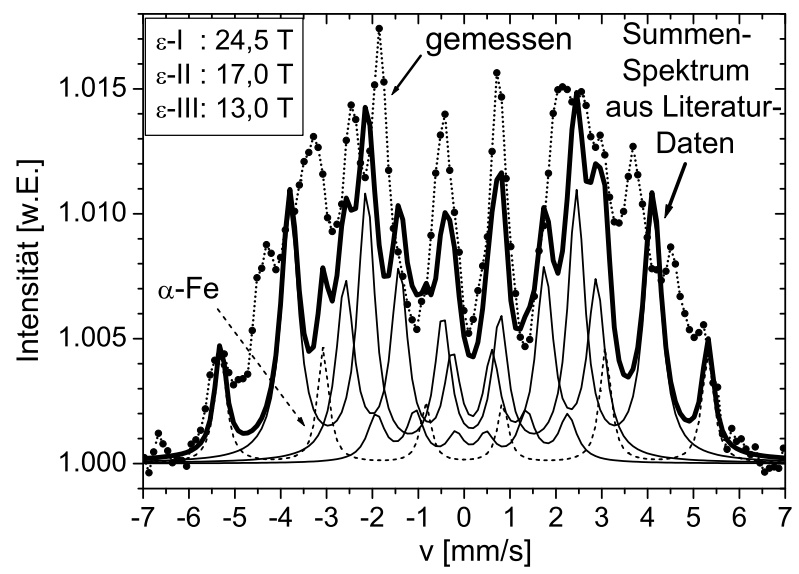

Abbildung 5.10: Vergleich des gemessenen $\varepsilon$-CarbidSpektrums mit den angegebenen Literaturdaten. Die Intensität wurde der Deutlichkeit halber so gewählt, dass die Anpassungsfehler zu erkennen sind.

der Unterspektren sind in Tab. 5.2 aufgeführt. In das gemessene Spektrum (s. Abb. 5.9p sind Hyperfeinfelder (ca. 13,0 T, 17,0 T und 24,5 T) der $\varepsilon$-Carbid-Daten der Literatur eingefügt (Abb. 5.10). Diese zeigen deutliche Diskrepanz, vor allem in der Position bzw. HF-Aufspaltung der Sextetts. Übereinstimmung herrscht bei dem 17,0 T-Unterspektrum. Der 27,0 T-Wert entspricht in etwa dem Hyperfeinfeld des $\varepsilon^{\prime}$-Carbid, das nur einen Fe-Platz aufweist [63|. Jedoch weichen auch in der Literatur die Werte stark

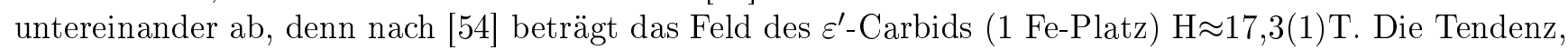


Tabelle 5.2: Hyperfeinfelder und Kohlenstoffgehalt des $\varepsilon-\mathrm{Fe}_{x} \mathrm{C}$.

\begin{tabular}{|l||c|l|l|}
\hline & Zusammensetzung & at.\% C & HF-Feld [T] \\
\hline \hline 1 C-Nachbar & $\mathrm{Fe}_{6} \mathrm{C}$ & 14,29 & $\sim 27,0$ \\
\hline 2 C-Nachbarn & $\mathrm{Fe}_{3} \mathrm{C}$ & 25,0 & $\sim 22,0$ \\
\hline 3 C-Nachbarn & $\mathrm{Fe}_{2} \mathrm{C}$ & 33,33 & $\sim 19,0$ \\
\hline
\end{tabular}

dass mit steigender Anzahl $n$ der C-Nachbarn in Fe-C-Verbindungen das Hyperfeinfeld linear nach dem Gesetz

$$
H(n)=H_{0}-n \cdot K \quad, \quad \text { mit }[K]=T
$$

sinkt, ist bekannt und intensiv untersucht worden [56,191].

\subsection{Pulsserie bei 0,1 bar Methan}

In der zweiten Serie wurde Eisen mit einer Pulszahl von $4 \times 4$ bis $16 \times 16$ und $\mathrm{p}\left(\mathrm{CH}_{4}\right)=0,1$ bar bestrahlt. Die Energiedichte beträgt für alle Pulsserien $4 \mathrm{~J} / \mathrm{cm}^{2}$. Sowohl das RBS-Spektrum (Abb. 5.11) als auch das

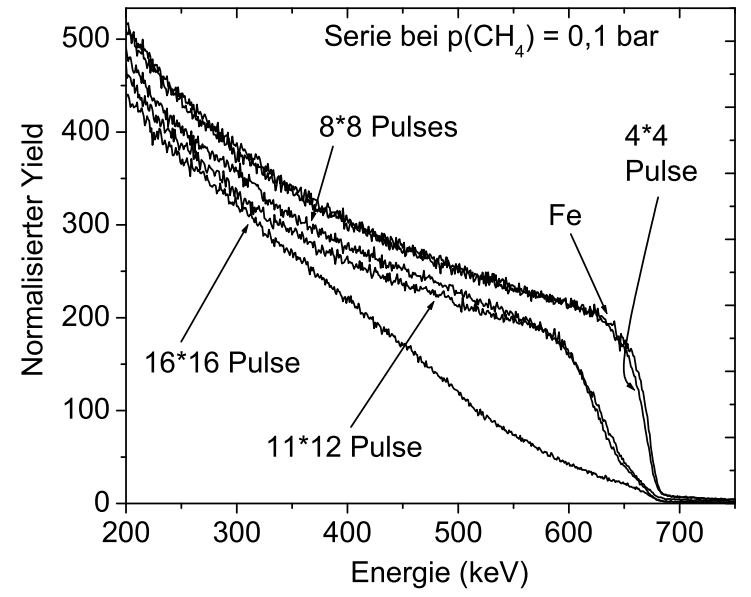

Abbildung 5.11: RBS-Spektren der Pulsserie bei $\mathrm{p}\left(\mathrm{CH}_{4}\right)=0,1$ bar.

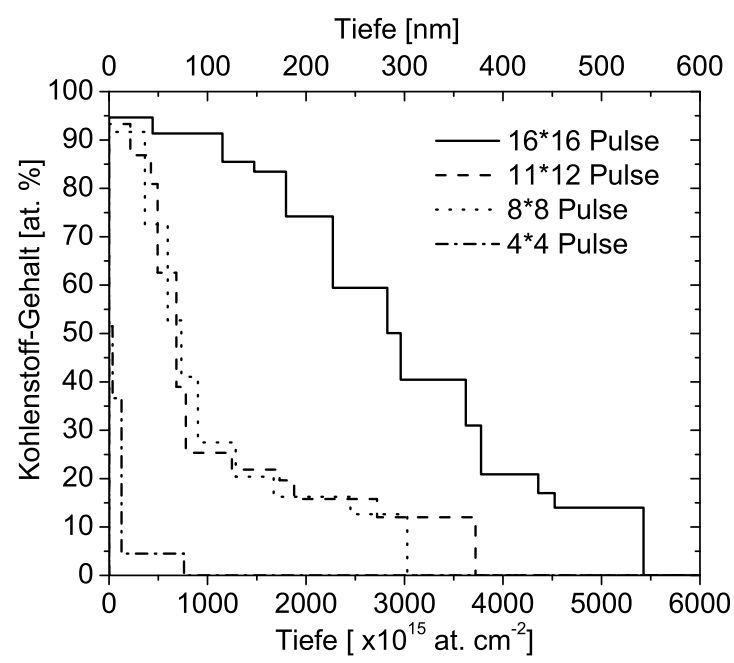

Abbildung 5.12: Kohlenstoffprofil der Pulsserie bei $\mathrm{p}\left(\mathrm{CH}_{4}\right)=0,1$ bar.

WiNDF-Kohlenstoffprofil (Abb. 5.12) zeigen einen enormen Anstieg des Kohlenstoffs auf der Oberfläche mit steigender Pulszahl. Je mehr Kohlenstoff in der Oberfläche inkorporiert ist, desto schwärzer ist diese. Die XRD-Spektren sind in den Abb 5.13 und 5.14 zu sehen: Die Spektren unterscheiden sich durch ihre Informationstiefe. Diese beträgt bei einem Einfallswinkel von $1^{\circ}$ ca. $150 \mathrm{~nm}$ bzw. bei $5^{\circ}$ ca. $750 \mathrm{~nm}$. Die Bildung des $\varepsilon$-Carbid beginnt bei $8 \times 8$ Pulsen. Nur bei der höchsten Pulszahl von $16 \times 16$ sind Reflexe von $\theta$-Carbid präsent, welche eigentlich nur bei hohen Drücken ab ca. 1-2 bar auftreten. In Abb. 5.16 ist das Spektrum der Probe dargestellt, die mit $16 \times 16$ Pulsen bestrahlt wurde. Der $\theta$-Gehalt steigt mit zunehmender Tiefe (Einfallswinkel) an. Der vergrößerte Ausschnitt in Abb. 5.15 zeigt die $\theta$-CarbidEntstehung anhand der ausgeprägtesten Peaks im low-angle-Bereich. Im Gegensatz zum $\varepsilon$-Carbid besitzt das $\theta$-Carbid eine kompliziertere Struktur, die zur Bildung mehr Zeit bzw. eine verminderte Kühlrate beansprucht, welche in größerer Tiefe auch zu erwarten ist [14].

Das Mössbauerspektrum (Abb. 5.17) bzw. die Flächenanteile der Phasen (Abb. 5.18) bestätigen die Beobachtung der Phasenbildung im XRD-Spektrum. Bei $4 \times 4$ Pulsen bildet sich $\gamma-\mathrm{Fe}(\mathrm{C})$. Bei Erhöhung der Pulszahl setzt ab $8 \times 8$ Pulsen die Bildung von $\varepsilon$-Carbid ein. Bei niedrigem Druck entsteht überwiegend $\varepsilon$-Carbid. Ein sehr wichtiger Punkt hierbei ist die Ursache für die Entwicklung des Kohlenstoffs auf der Oberfläche: Je höher die Pulszahl $n$ gewählt wird, desto mehr Kohlenstoff bildet sich auf der Oberfläche. 


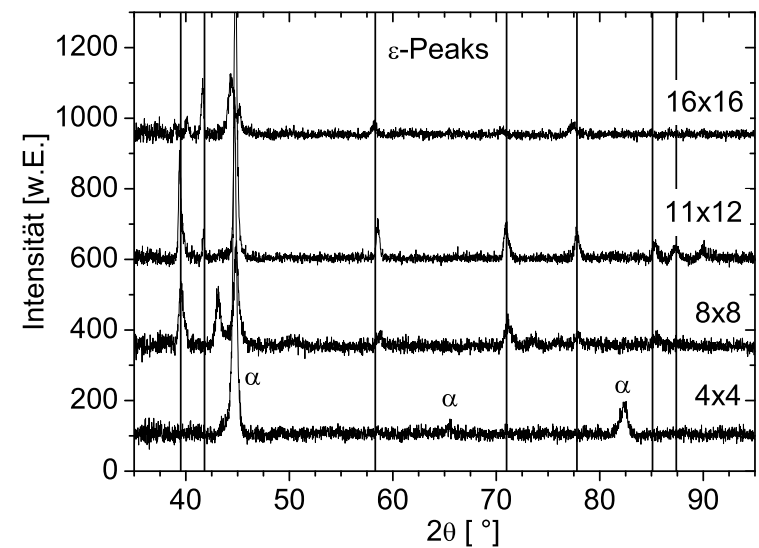

Abbildung 5.13: XRD-Spektrum der Pulsserie bei $\mathrm{p}\left(\mathrm{CH}_{4}\right)=0,1$ bar. Der Einfallswinkel der Röntgenstrahlung ist $1^{\circ}(\approx 150 \mathrm{~nm}$ Informationstiefe).

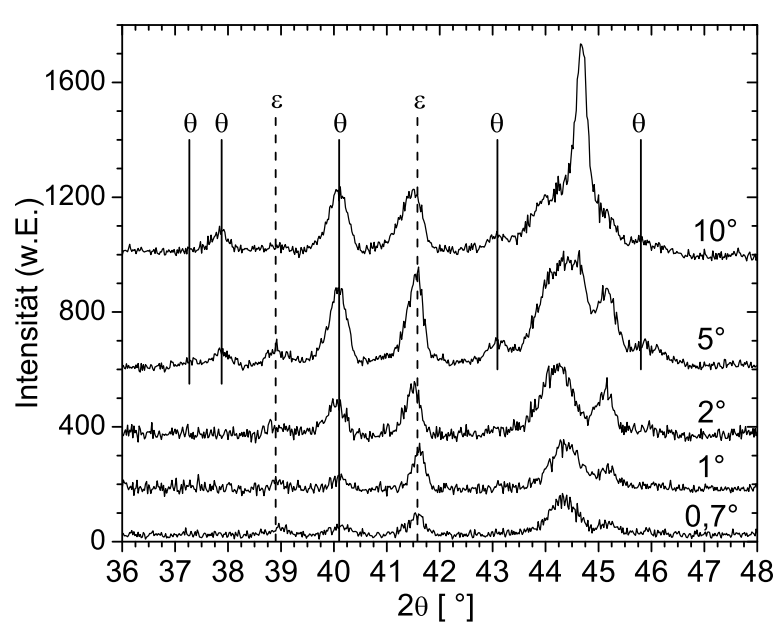

Abbildung 5.15: XRD-Teil-Spektren der Pulsserie bei $\mathrm{p}\left(\mathrm{CH}_{4}\right)=0,1$ bar.

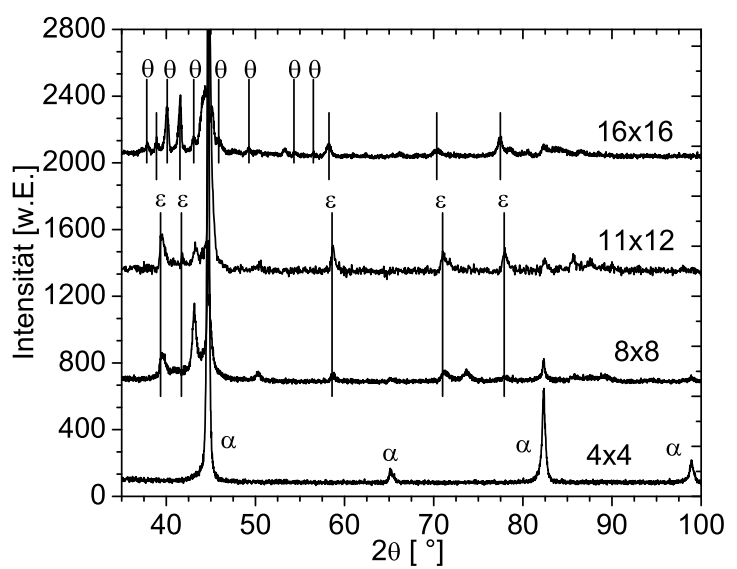

Abbildung 5.14: XRD-Spektrum der Pulsserie bei $\mathrm{p}\left(\mathrm{CH}_{4}\right)=0,1$ bar. Der Einfallswinkel der Röntgenstrahlung ist $5^{\circ}$ ( $\approx 750 \mathrm{~nm}$ Informationstiefe).

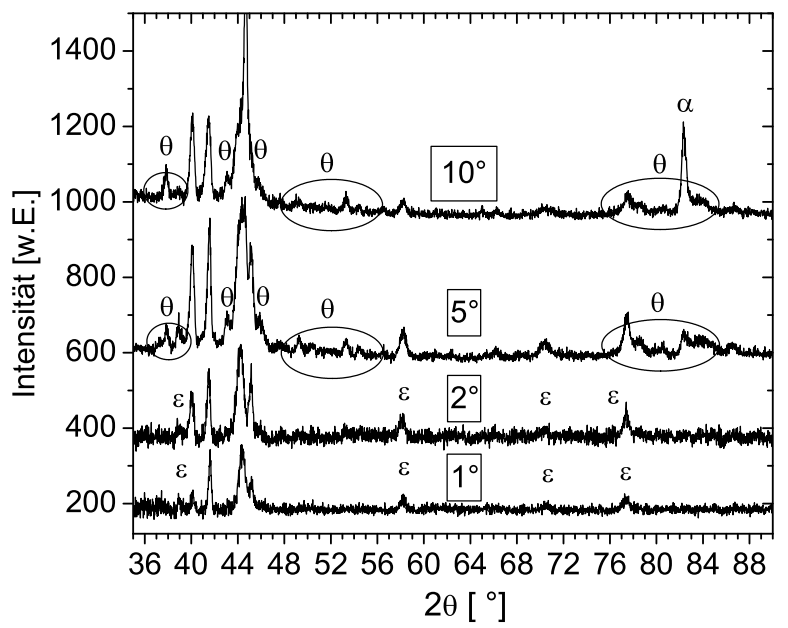

Abbildung 5.16: XRD-Spektren der Pulsserie bei $\mathrm{p}\left(\mathrm{CH}_{4}\right)=0,1$ bar. Die ovalen Markierungen zeigen der Übersichtlichkeit halber die typischen $\theta$-Regionen.

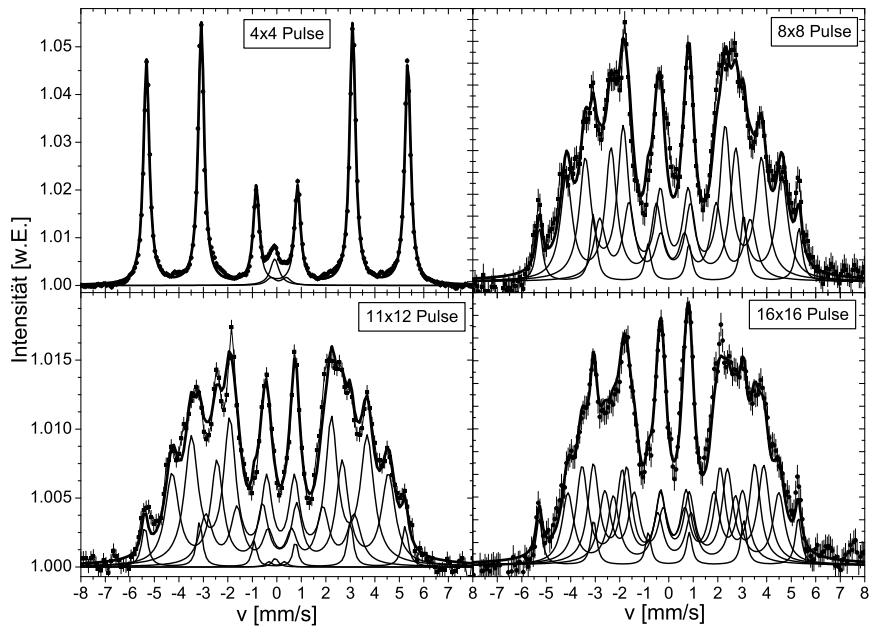

Abbildung 5.17: Mössbauerspektren der Pulsserie bei $\mathrm{p}\left(\mathrm{CH}_{4}\right)=0,1$ bar.

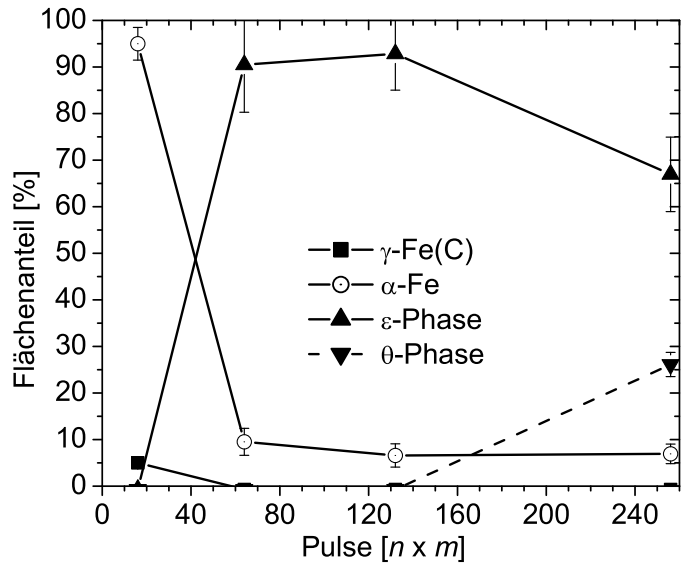

Abbildung 5.18: Flächenanteil der verschiedenen Phasen der Pulsserie bei $\mathrm{p}\left(\mathrm{CH}_{4}\right)=0,1$ bar. 
Bei $4 \times 4$ Pulsen ist noch keine Entwicklung zu erkennen. Aber: Die Puls-Parameter sind für sich genommen bei allen Bestrahlungen gleich.

\subsection{Pulsserie bei 4 bar Methan}

Die Pulsserie bei $\mathrm{p}\left(\mathrm{CH}_{4}\right)=4$ bar besitzt eine gänzlich andere Tendenz der Phasenbildung als die Serie bei $\mathrm{p}\left(\mathrm{CH}_{4}\right)=0,1$ bar. Abb. 5.19 zeigt im Gegensatz zum Spektrum in Abb. 5.11 keinen Kohlenstoff auf der Oberfläche, jedoch liegt ein vergleichbar hoher Kohlenstoff-Anteil in der Bulk-Phase vor. In Abb. 5.20 sind die Tiefenprofile der Serie dargestellt. Bei niedrigen Pulszahlen $(4 \times 4$ und $8 \times 8)$ wird im Vergleich

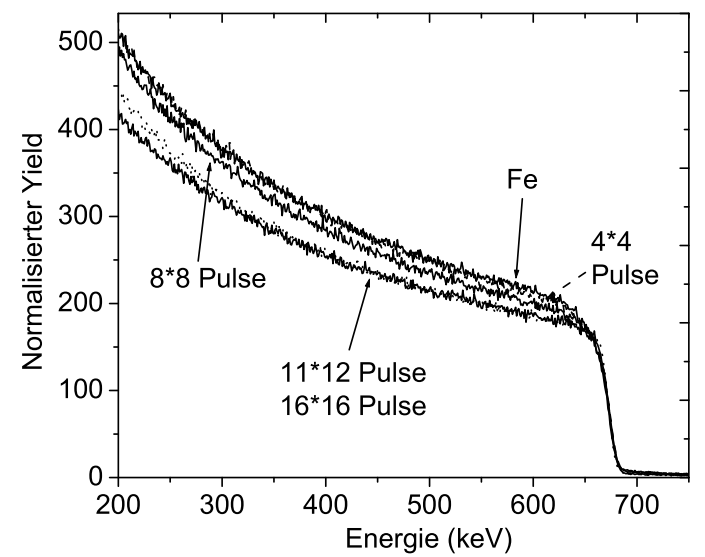

Abbildung 5.19: RBS-Spektrum der Pulsserie bei $\mathrm{p}\left(\mathrm{CH}_{4}\right)=4$ bar.

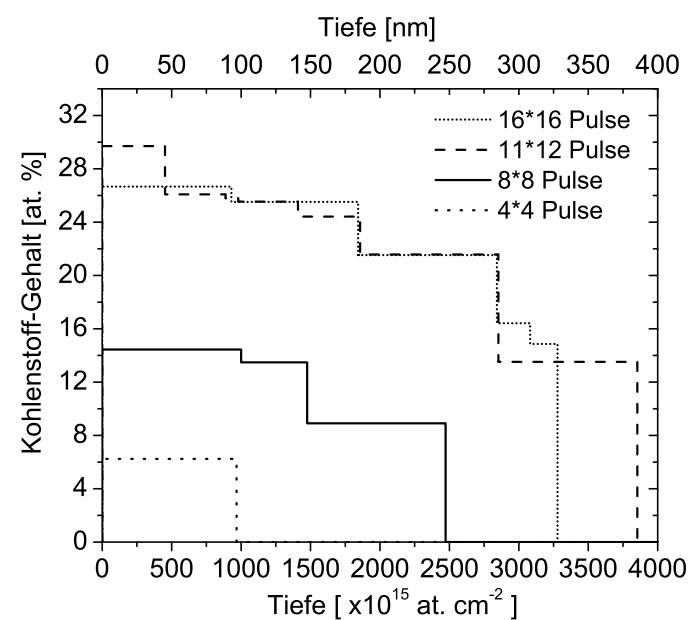

Abbildung 5.20: Kohlenstoffprofil der Pulsserie bei $\mathrm{p}\left(\mathrm{CH}_{4}\right)=4$ bar.

zu 0,1 bar- oder 1 bar-Proben am meisten $\gamma$-Fe(C) gebildet. Nur die $8 \times 8$-Probe zeigt im CEM-Spektrum

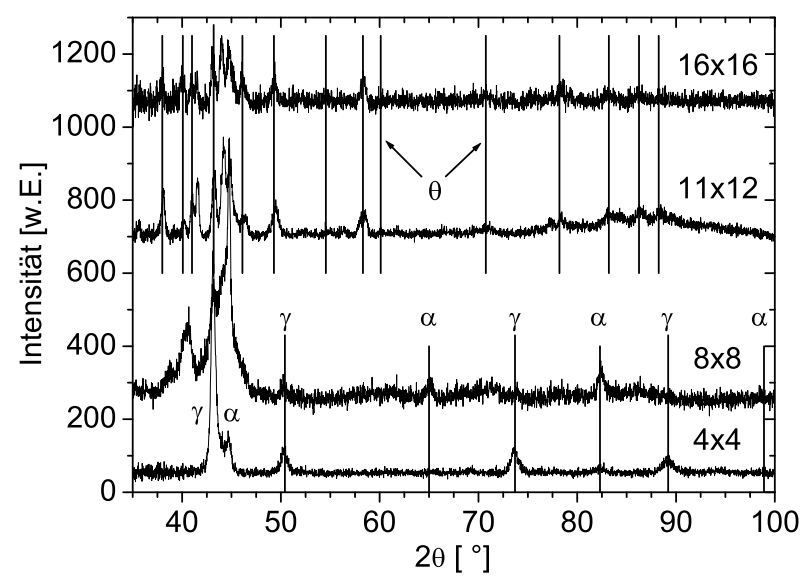

Abbildung 5.21: XRD-Spektrum der Pulsserie bei $\mathrm{p}\left(\mathrm{CH}_{4}\right)=4$ bar. Der Einfallswinkel der Röntgenstrahlung ist $1^{\circ}(\approx 150 \mathrm{~nm}$ Informationstiefe)

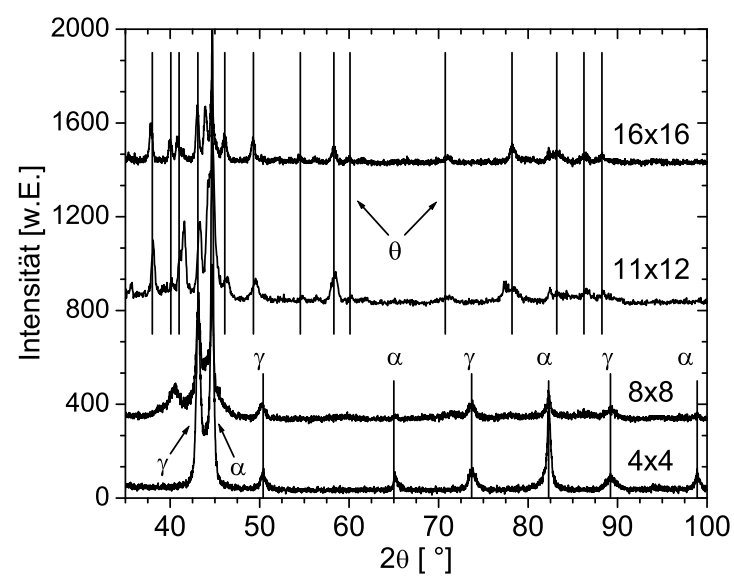

Abbildung 5.22: XRD-Spektrum der Pulsserie bei $\mathrm{p}\left(\mathrm{CH}_{4}\right)=4$ bar. Der Einfallswinkel der Röntgenstrahlung ist $5^{\circ}(\approx 750 \mathrm{~nm}$ Informationstiefe)

(Abb. 5.23 eine typische Sextett-Konstellation, die dem Martensit $\left(\alpha^{\prime}-\mathrm{Fe}\right)$ zugeordnet werden kann. Die maximale Kohlenstoff-Konzentration im $\alpha^{\prime}$-Fe beträgt wie im $\gamma$-Fe(C) (bei $1153^{\circ} \mathrm{C}$ ) 8,3 at.\% [44 28]. Auch in den XRD-Spektren (Abb. 5.21 und 5.22 werden bei $4 \times 4$ und $8 \times 8$ Pulsen nur die Peaks des $\alpha\left({ }^{\prime}\right)$-Fe und $\gamma$ - $\mathrm{Fe}(\mathrm{C})$ beobachtet. Die Bildung des $\theta$-Carbids beginnt bei $11 \times 12$ Pulsen, ohne dass die $\varepsilon$-Phase auftritt. Bei hohem Druck ist die dominierende Phase das $\theta$-Carbid. 


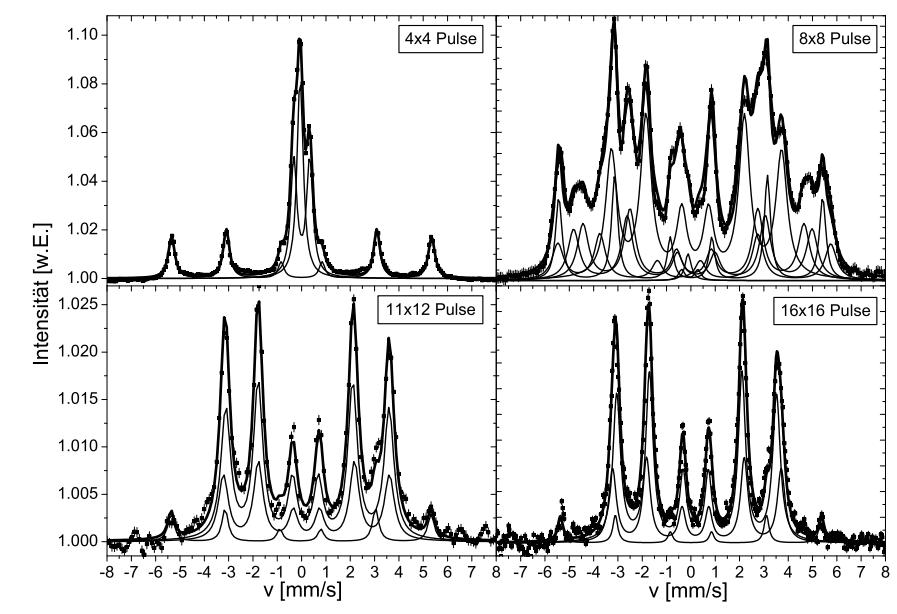

Abbildung 5.23: Mössbauerspektrum der Pulsserie bei $\mathrm{p}\left(\mathrm{CH}_{4}\right)=4$ bar.

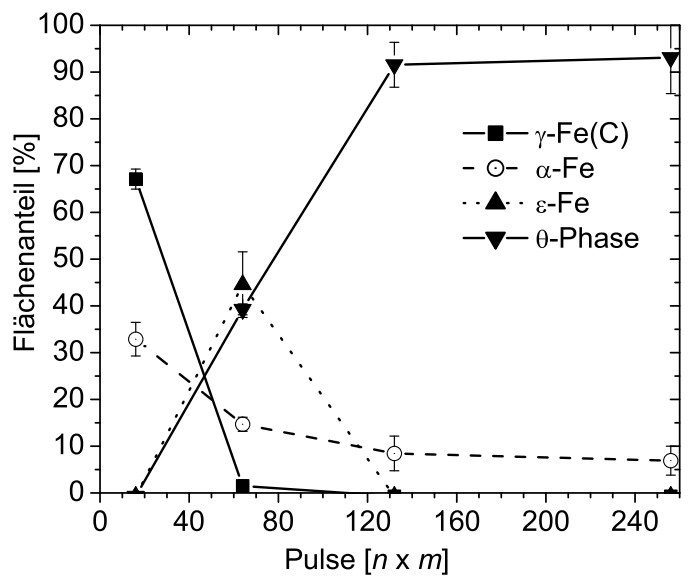

Abbildung 5.24: Flächenanteil der Fe-C-Phasen der Pulsserie bei $\mathrm{p}\left(\mathrm{CH}_{4}\right)=4$ bar.

\subsection{Pulsserie bei 1 bar Methan}

Die Serie bei $\mathrm{p}\left(\mathrm{CH}_{4}\right)=1$ bar liegt in der Entwicklung der Phasen zwischen den Serien bei $\mathrm{p}\left(\mathrm{CH}_{4}\right)=0,1$ und 4 bar, wie man schon bei der Druckserie beobachten konnte. Bei $4 \times 4$ Pulsen bildet sich die $\gamma$-Phase. Im

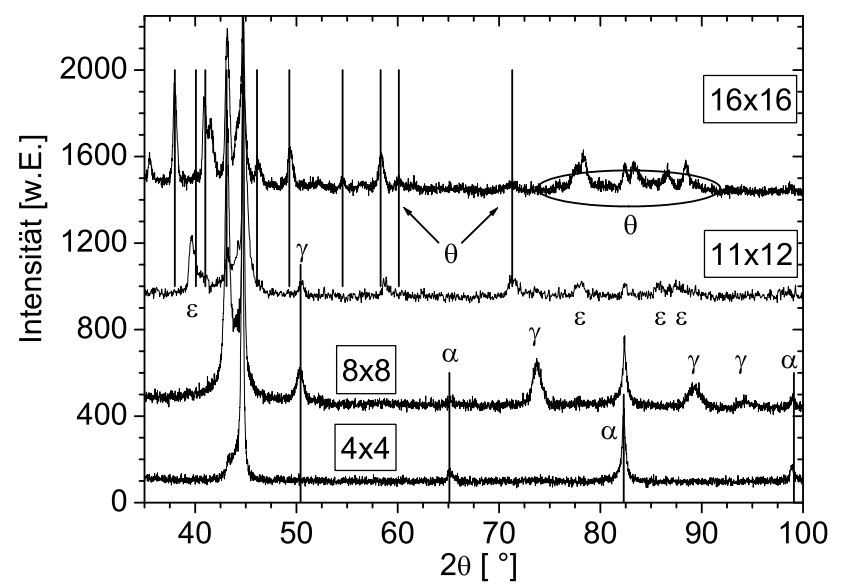

Abbildung 5.25: XRD-Spektren der Pulsserie bei $\mathrm{p}\left(\mathrm{CH}_{4}\right)=1$ bar.

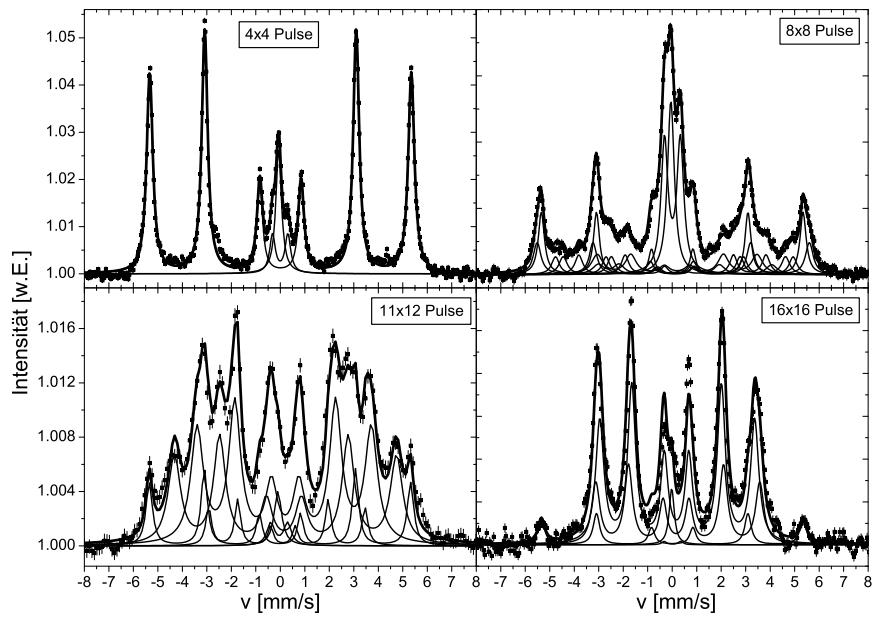

Abbildung 5.26: Mössbauerspektren der Pulsserie bei $\mathrm{p}\left(\mathrm{CH}_{4}\right)=1$ bar.

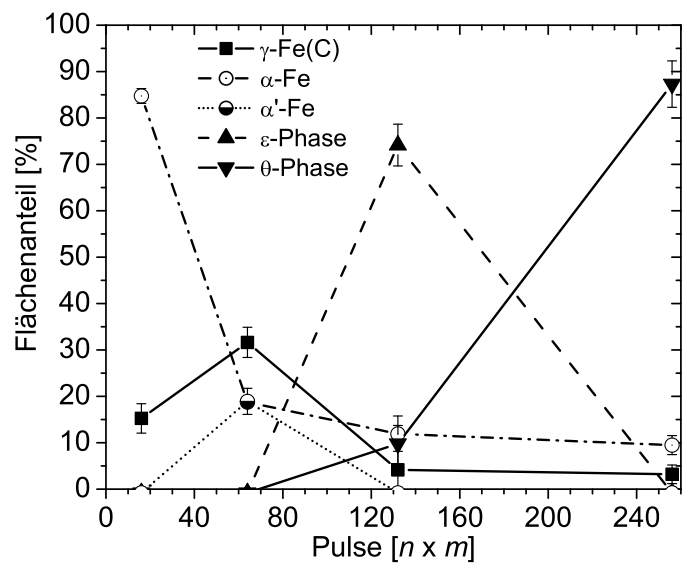

Abbildung 5.27: Flächenanteil der verschiedenen Phasen der Pulsserie bei $\mathrm{p}\left(\mathrm{CH}_{4}\right)=1$ bar. 
Gegensatz zu den beiden anderen Druckserien bildet sich bei $8 \times 8$ Pulsen ein $\alpha\left({ }^{\prime}\right) / \varepsilon$-Gemisch. Bei $11 \times 12$ Pulsen bildet sich ein wenig $\theta$-Carbid, aber der größte Anteil ist $\varepsilon$-Carbid, das komplett in $\theta$-Carbid bei $16 \times 16$ Pulsen übergeht. Auch diese Serie zeigt, dass bei der höchsten Pulszahl von $16 \times 16$ schließlich nur eine Phase auftritt.

\subsection{Vergleich der Pulsserien}

Vergleicht man die Pulsserien bei 0,1,1 und 4 bar, so ist ein klares Bildungsschema für die $\theta$ - und $\varepsilon$-Carbide erkennbar. Die Mössbauerspektren für die Bestrahlung bei $4 \times 4$-Pulsen ist in Abb. 5.28 aufgezeigt. Der errechnete Kohlenstoffgehalt ist in Tab. 5.3 aufgelistet. Mit steigendem Druck steigt nicht

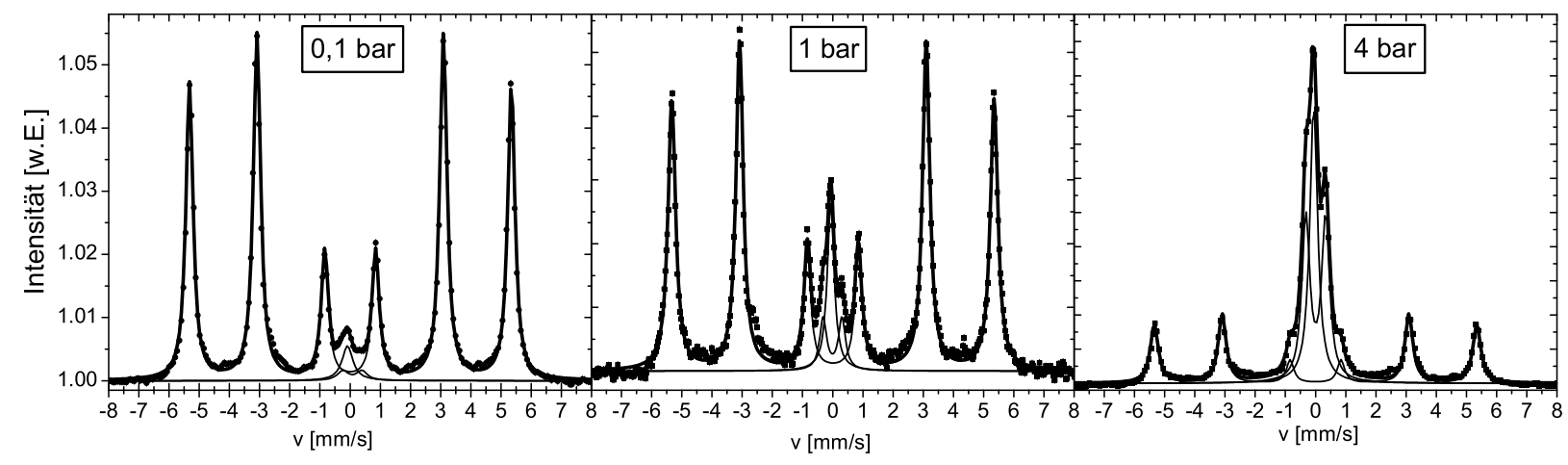

Abbildung 5.28: CEM-Spektren der mit $4 \times 4$ Pulsen bestrahlten Proben bei $\mathrm{p}\left(\mathrm{CH}_{4}\right)=0,1 / 1$ und 4 bar.

nur der Kohlenstoffgehalt im Austenit, sondern auch der relative Anteil in Probe. Bei der 4 bar hat der Kohlenstoff im $\gamma$-Fe(C) die Sättigungsgrenze von ca. 9 at.\% erreicht. Der RBS-Kohlenstoff-Anteil liegt stets etwas über dem CEMS-Anteil (s. Kap. 5.13). Der Fehler des Kohlenstoffgehaltes ist aufgrund des

Tabelle 5.3: Mithilfe von CEMS und RBS bestimmter Kohlenstoffgehalt im $\gamma-\mathrm{Fe}(\mathrm{C})$ und Gesamtanteil der $4 \times 4$ Puls-Proben.

\begin{tabular}{|c|c||c|c|}
\hline Druck [bar] & $\begin{array}{c}\text { C-Anteil in } \\
\gamma-\mathrm{Fe}(\mathrm{C}) \text { [at.\%] }\end{array}$ & $\begin{array}{c}\text { C-Anteil aus CEMS } \\
\text { gesamt [at.\%] }\end{array}$ & $\begin{array}{c}\text { C-Anteil aus RBS } \\
\text { gesamt [at.\%] }\end{array}$ \\
\hline \hline 0,1 & $5,64(100)$ & $0,273(50)$ & - \\
\hline 1 & $6,00(390)$ & $0,915(600)$ & $2(1)$ \\
\hline 4 & $8,26(51)$ & $5,54(53)$ & $9(2)$ \\
\hline
\end{tabular}

geringen Vorkommens relativ hoch und sinkt bei steigendem Anteil. Bei hohen Pulszahlen dominiert bei $\mathrm{p}\left(\mathrm{CH}_{4}\right)=0,1$ bar das $\varepsilon$-Carbid; bei $\mathrm{p}\left(\mathrm{CH}_{4}\right)=4$ bar bildet sich ausschließlich das $\theta$-Carbid. In den mittleren Pulsbereichen bilden sich Mischungen einzelner Phasen oder Carbid-Vorstufen wie $\alpha^{\prime}$-Fe oder $\gamma$-Fe(C), die noch genug Kohlenstoff aufnehmen können. Erst die hohen Pulszahlen führen zur Sättigung der Kohlenstoffaufnahme von ca. 25 at.\%. Bei der Bildung des $\theta$-Carbids bei hohem Druck und Pulszahlen ist die Vorstufe in keinem Fall $\varepsilon$-Carbid. Ebenso geht der Bildung von $\varepsilon$-Carbid bei niedrigem Druck und hohen Pulszahlen kein $\theta$-Carbid voraus.

Mithilfe der CXMS-Messungen konnten die Flächenanteile des $\varepsilon$ - und des $\theta$-Carbids der bestrahlten Proben bei $\mathrm{p}\left(\mathrm{CH}_{4}\right)=0,1$ bar (Tab. 5.4) und $\mathrm{p}\left(\mathrm{CH}_{4}\right)=4$ bar (Tab. 5.5) errechnet werden: Mit steigender Pulszahl wächst in der $\mathrm{p}\left(\mathrm{CH}_{4}\right)=0,1$ bar-Serie der Anteil an $\varepsilon$-Carbid auf Kosten des $\gamma$-Fe(C). Bei $16 \times 16$ Pulsen ist zusätzlich ein hoher Anteil von ca. $10 \%$ zu verzeichnen, das $\varepsilon$-Carbid liegt nur noch zu 1,9\% vor. Die gleiche Tendenz ist in der $\mathrm{p}\left(\mathrm{CH}_{4}\right)=4$ bar-Serie zu beobachten. Anstelle des $\varepsilon$-Carbids wird $\theta$ Carbid gebildet. Der Kohlenstoff-Eintrag beginnt bei niedrigem Druck bereits ab $8 \times 8$ Pulsen und ist etwas höher als bei hohem Druck. In den CEM-Spektren ist kaum $\gamma$-Fe $(\mathrm{C})$ auflösbar, in den CXM-Spektren ist dagegen ein beträchtlicher Anteil relativ zu den anderen Phasen vorhanden. Um die Carbide zu bilden, 
Tabelle 5.4: CXMS-Flächenanteil der Serie bei $\mathrm{p}\left(\mathrm{CH}_{4}\right)=0,1$ bar.

Tabelle 5.5: CXMS-Flächenanteil der Serie bei $\mathrm{p}\left(\mathrm{CH}_{4}\right)=4$ bar.

\begin{tabular}{|c|c|c|c|c|c|c|c|}
\hline Pulse & $\mathrm{Fe}[\%]$ & $\gamma-\mathrm{Fe}(\mathrm{C})[\%]$ & Carbide [\%] & Pulse & Fe [\%] & $\gamma-\mathrm{Fe}(\mathrm{C})[\%]$ & Carbide [\%] \\
\hline $4 \times 4$ & 100 & - & - & $4 \times 4$ & $97,14(261)$ & $2,86(-)$ & - \\
\hline $8 \times 8$ & $93,18(174)$ & $4,2()$ & $\varepsilon: 2,62(-)$ & $8 \times 8$ & $95,08(167)$ & $4,92(-)$ & - \\
\hline $11 \times 12$ & $91,95(176)$ & $3,43(-)$ & $\varepsilon: 4,62(-)$ & $11 \times 12$ & $93,11(296)$ & $2,56(-)$ & $\theta: 4,33(273)$ \\
\hline \multirow{2}{*}{$16 \times 16$} & \multirow{2}{*}{$86,21(195)$} & \multirow{2}{*}{$1,76(-)$} & \multirow{2}{*}{$\begin{array}{l}\varepsilon: 1,86(-) \\
\theta: 10,17(102)\end{array}$} & $16 \times 16$ & $86,89(126)$ & $3,79(162)$ & $\theta: 9,31(-)$ \\
\hline & & & & & & & \\
\hline
\end{tabular}

bedarf es einen Mindest-Anteil Kohlenstoff. Wird dieses Minimum in großen Tiefen unterschritten, bildet sich $\gamma-\mathrm{Fe}(\mathrm{C})$.

Die Unterschiede der Bildungssequenzen der einzelnen Phasen in Abhängigkeit der Pulsanzahl kann man wie folgt zusammenfassen:

- $\mathrm{p}\left(\mathrm{CH}_{4}\right)=0,1$ bar: niedriger Anteil $\gamma$-Fe(C) $\longrightarrow \varepsilon$-Carbid

- $\mathrm{p}\left(\mathrm{CH}_{4}\right)=1$ bar: mittlerer Anteil $\gamma$-Fe $(\mathrm{C}) \longrightarrow \varepsilon$-Carbid $\longrightarrow \theta$-Carbid

- $\mathrm{p}\left(\mathrm{CH}_{4}\right)=4$ bar: hoher Anteil $\gamma$-Fe $(\mathrm{C}) \longrightarrow \alpha^{\prime}$-Fe $\longrightarrow \theta$-Carbid

Ein weiterer wichtiger Aspekt ist, wie und ob der partielle- bzw. totale $\mathrm{CH}_{4}$-Druck eine Rolle spielt. Dazu wurden 2 Proben bei $11 \times 12$ und $\mathrm{p}\left(\mathrm{CH}_{4}\right)=0,1$ bar mit dem Rohstrahl bearbeitet (Abb. 5.29 und 5.30). Die Methan-Atmosphäre wurde zusätzlich mit Argon versetzt. Erwartungsgemäß bildet sich bei $\mathrm{p}\left(\mathrm{CH}_{4}\right)=0,1$

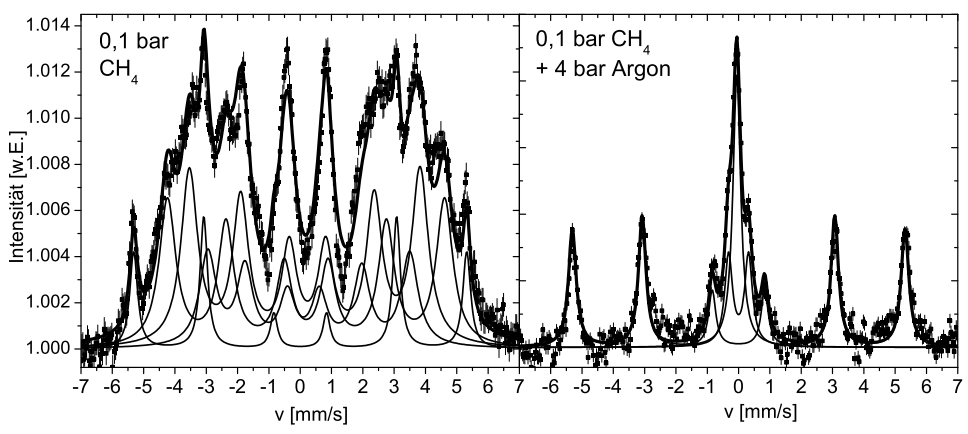

Abbildung 5.29: CEM-Spektren der Proben: $4 \mathrm{~J} / \mathrm{cm}^{2}$, Raw Beam, $11 \times 12$ Pulse, $\mathrm{p}\left(\mathrm{CH}_{4}\right)=0,1$ bar. Das linke Gesamt-Spektrum enthält typische $\varepsilon$-Unterspektren. Rechtes Spektrum: Bei Zugabe von 4 bar Ar bildet sich weder $\varepsilon$ - noch $\theta$-Carbid, sondern $\gamma$-Fe(C).

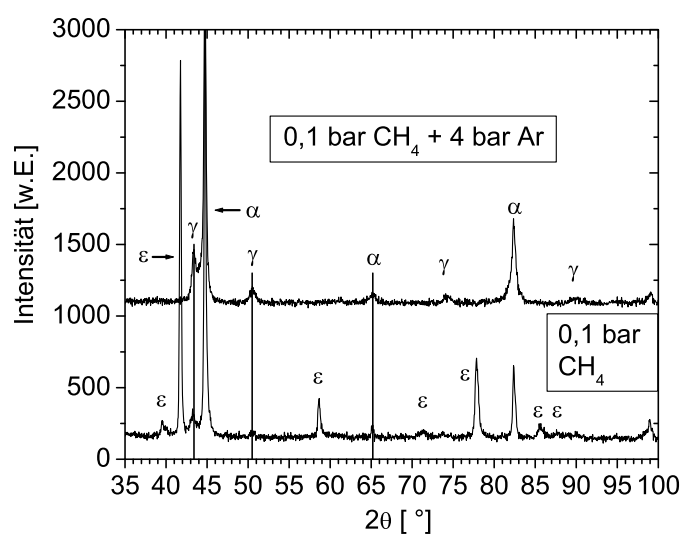

Abbildung 5.30: XRD-Spektren. Die Probe bei $\mathrm{p}\left(\mathrm{CH}_{4}\right)=0,1$ bar $+\mathrm{p}(\mathrm{Ar})=4$ bar weist nur $\gamma$ und $\alpha$-Fe auf, was Abb. 5.29 bestätigt, während bei $\mathrm{p}\left(\mathrm{CH}_{4}\right)=0,1$ bar das $\varepsilon$-Carbid entsteht.

bar ohne Ar-Zusatz $\varepsilon$-Carbid und etwas $\alpha$ - und $\gamma$-Fe. Bei der nächsten Proben-Bestrahlung erhöht man nun den Gesamtdruck durch Zugabe von 4 bar Ar auf $\mathrm{p}_{\text {ges }}=4,1$ bar. Betrachtet man nur den Partialdruck von $\mathrm{p}\left(\mathrm{CH}_{4}\right)^{\text {part }}=0,1$ bar, so würde man die Entstehung von $\varepsilon$-Carbid erwarten. Andererseits beträgt der Gesamtdruck $\mathrm{p}_{\text {ges }}=4,1$ bar, bei dem sich üblicherweise $\theta$-Carbid bildet.

Die Spektren der $\mathrm{p}(\mathrm{Ar})=4$ bar-Probe zeigen überraschenderweise weder $\varepsilon$ - noch $\theta$-Carbid, sondern $\gamma$ $\mathrm{Fe}(\mathrm{C})$. Die CEMS-Analyse deutet auf einen Gesamt-Kohlenstoffgehalt von nur 2,4(10) at.\% hin, während die Probe, die nur in Methan bestrahlt wurde, einen C-Gehalt von ca. 21(2) at.\% aufweist und im Bereich der üblichen $\varepsilon$-Carbide liegt. Das CEM-Spektrum ähnelt der Probe bei $4 \times 4$ Pulse und $\mathrm{p}\left(\mathrm{CH}_{4}\right)=4$ bar (s. Abb. 5.28). Es wurde nur 1 Bestrahlung in Ar-Atmosphäre durchgeführt, als dass man sagen könnte, wie nun der Partialdruck des Ar die Phasenbildung beeinflusst. Das Ergebnis zeigt aber, dass keine der beiden Extremalphasen gebildet wird. Vielmehr behindert das Argon den Carburisierungsprozess und resultiert 
in einer sehr geringen Kohlenstoffaufnahme, die nur zur Bildung von $\gamma$-Fe(C) führt. Dieses Beispiel zeigt, dass sowohl der Gesamtdruck als auch die Atomspezies die Plasmadynamik beträchtlich ändern kann (s.Kap. 4.4.1).

\subsection{Vergleich zwischen Roh- und homogenisiertem Strahl}

Zwei weitere Proben wurden bei $H \approx 3,5 \mathrm{~J} / \mathrm{cm}^{2}$ und $11 \times 12$ Pulsen bestrahlt. Das CEM-Spektrum in Abb. 5.32 zeigt die markanten Unterschiede bei der Bestrahlung mit dem homogenisierten (links) und dem Rohstrahl (rechts). Zieht man für die Probe, die mit dem Roh-Strahl bearbeitet wurde, die Energieserie (s. Abb. 5.37) in Betracht, so ist ersichtlich, dass man sich bei einer Energiedichte von ca. $H=3,5 \mathrm{~J} / \mathrm{cm}^{2}$ gerade am Übergang zur Bildung des $\varepsilon$-Carbids befindet, während für den homogenisierten Strahl 3,5 $\mathrm{J} / \mathrm{cm}^{2}<H \leq 4 \mathrm{~J} / \mathrm{cm}^{2}$ erforderlich sind. Für den homogenisierten Strahl ist mit 3,5 J/ $\mathrm{cm}^{2}$ diese Schwelle

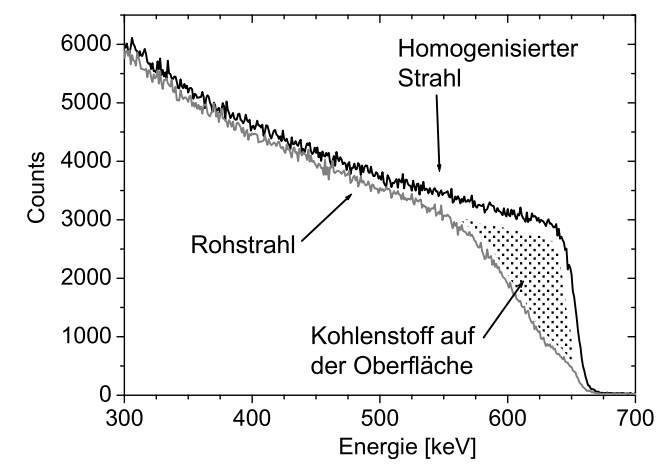

Abbildung 5.31: RBS-Spektrum der Proben, die bei ca. $3,5 \mathrm{~J} / \mathrm{cm}^{2}$ und $11 \times 12$ Pulsen mit dem homogenisierten und dem Rohstrahl bearbeitet wurden.

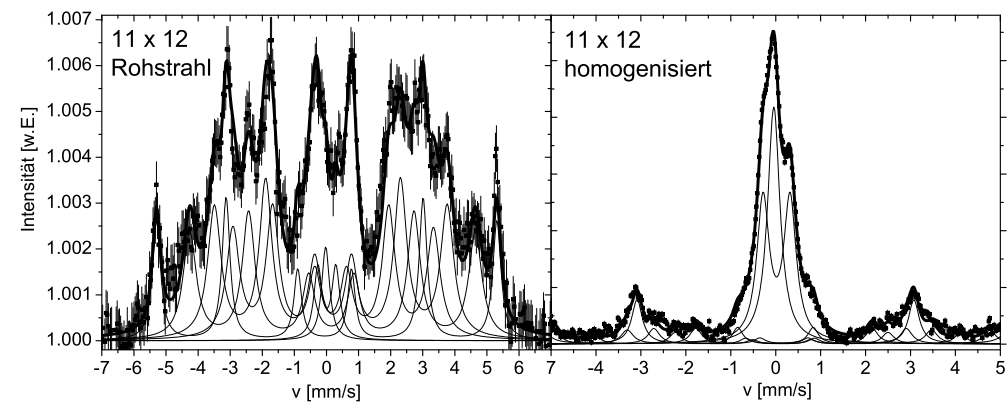

Abbildung 5.32: CEM-Spektrum der Proben, die mit $11 \times 12$ Pulsen bei ca. $3,5 \mathrm{~J} / \mathrm{cm}^{2}($ !) bestrahlt wurden. Links ist das Spektrum der Probe bei Bestrahlung mit dem homogenisierten, rechts mit dem Roh-Strahl dargestellt.

knapp unterschritten, was sich an der Bildung des $\gamma$-Fe(C) zeigt. Auch bei der (nicht dargestellten) $8 \times 8$ Puls-Probe bildet sich im Gegensatz zu der Bestrahlung bei $4 \mathrm{~J} / \mathrm{cm}^{2} \gamma-\mathrm{Fe}(\mathrm{C})$. Geht man jedoch zum Rohstrahl bei $11 \times 12$ Pulsen über, so reicht diese Energiedichte aus, $\varepsilon$-Carbid in ausreichender Menge zu erzeugen. Der Grund ist, dass die gemessene Energie der gemittelte Wert über die Spot-Fläche ist. Beim homogenisierten Strahl ist das Laser-Profil eben sehr gleichförmig. Im Rohstrahl hingegen überschreitet das Zentrum des Profils offenbar den Energie-Mittelwert, während dieser in den Randbereichen unterschritten wird.

Man nehme an, dass der Rohstrahl-Laserspot eine mittlere Energiedichte von $H=3,5 \mathrm{~J} / \mathrm{cm}^{2}$ habe und eine Fläche von $5 \times 5 \mathrm{~mm}^{2}$ bedecke. Auf das als quadratisch angenommene Zentrum, das die Hälfte des Spots ausmacht, fällt -stark vereinfacht- ein mittleres $H=3,6 \mathrm{~J} / \mathrm{cm}^{2}$, auf den Rand $H=3,4 \mathrm{~J} / \mathrm{cm}^{2}$. Die Mäanderschritte des Laserspots betragen $\Delta x=5 \mathrm{~mm} / 11 \approx 0,455 \mathrm{~mm}$ bzw. $\Delta y=5 \mathrm{~mm} / 12 \approx 0,417$ mm. Das Zentrum der Kantenlänge $3,54 \times 3,54 \approx 12,5 \mathrm{~mm}^{2}$ führt somit eine effektive Anzahl von $n=$ $(5 \mathrm{~mm} / 11)^{-1} \cdot 3,54 \mathrm{~mm} \approx 7,8$ und $m=(5 \mathrm{~mm} / 12)^{-1} \cdot 3,54 \mathrm{~mm} \approx 8,5$, also $7,8 \times 8,5 \approx(11 \times 12) / 2$ Pulsen aus: In der Pulsserie bei $\mathrm{p}\left(\mathrm{CH}_{4}\right)=0,1$ bar (Abb. 5.17) enthält die $8 \times 8$-Probe $\varepsilon$-Carbid und ist von Kohlenstoff bedeckt. Die weitere Betrachtung der anderen Fläche wurde weggelassen, da diese wegen der kleineren Energiedichte anscheinend nicht zur Bildung des $\varepsilon$-Carbids beiträgt.

Die allgemeine Schwierigkeit der Laserbestrahlung liegt darin, für jede neue Probenserie genau $4 \mathrm{~J} / \mathrm{cm}^{2}$ zu erreichen, um so Reproduzierbarkeit zu gewährleisten. Fällt die Leistung des Laser etwas unter dieses Maximum, das mit dem homogenisierten Strahl noch erreicht werden kann, so unterschreitet man u.U. auch die Schwelle zur $\varepsilon$-Carbid-Bildung. Wie diese Messreihe zeigt, hängt die Bildung des $\varepsilon$-Carbids oder des $\gamma$-Fe $(\mathrm{C})$ empfindlich von der Schwellen-Energiedichte von ca. $3,6 \mathrm{~J} / \mathrm{cm}^{2}$ (bei $11 \times 12 \mathrm{Pulsen}$ ) ab. Die Kohlenstoffbildung wird nicht nur vom Überlapp der Laserpulse bestimmt, sondern auch von der effektiven Energiedichte, was auch in der nachfolgenden Energie-Serie beobachtet werden kann. 


\subsection{Energie-Serie}

Die Proben der Energieserie wurden unter folgenden Prozessbedingungen hergestellt: Dieses ist die einzige

Tabelle 5.6: Prozessparameter der Energieserie.

\begin{tabular}{|c|c|c|c|c|c|c|c|}
\hline $\mathrm{p}\left(\mathrm{CH}_{4}\right)$ & Pulse & \multicolumn{5}{|c|}{ Energiedichte $\left[\mathrm{J} / \mathrm{cm}^{2}\right]$} \\
\hline \hline 0,1 bar & $11 \times 12$ & 3,25 & 3,6 & 4,0 & 4,8 & 5,5 & 6,9 \\
\hline
\end{tabular}

Serie, die mit dem Raw Beam bestrahlt wurde. Die Energiedichte besitzt also kein homogenes Top-HatProfil. Die Konsequenz dieser Inhomogenität ist, dass Konvektionen eine wesentliche Rolle im Materialinsbesondere Kohlenstofftransport spielen (s. Kap.4.5). Das RBS-Spektrum in Abb.5.33zeigt die typische Niederdruck-Eigenschaft, einen enormen Kohlenstoff-Anteil auf der Oberfläche aufzuweisen. Der maximale

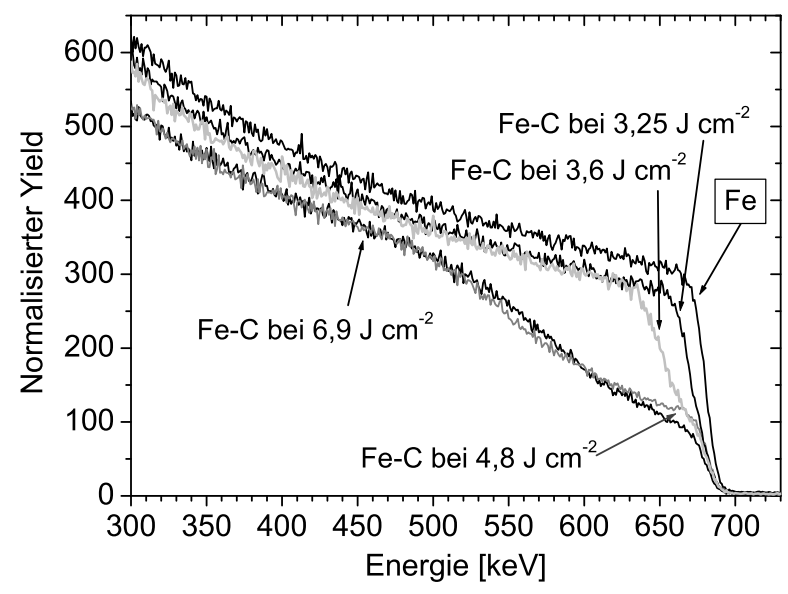

Abbildung 5.33: RBS-Spektrum der Energieserie bei $\mathrm{p}\left(\mathrm{CH}_{4}\right)=4$ bar und $11 \times 12$ Pulsen.

Kohlenstoffgehalt beträgt ca. 27 at.\% bei einer Energiedichte von $6,9 \mathrm{~J} / \mathrm{cm}^{2}$ und sinkt bis auf ca. 8 at.\% bei 3,25 at.\%. Die Schwärzung der Oberfläche steigt ebenfalls mit der Energiedichte. Die XRD-Spektren wurden bei einem Einfallswinkel von $1^{\circ}$ (entspricht ca. $150 \mathrm{~nm}$ Informationstiefe), $5^{\circ}$ (ca. $750 \mathrm{~nm}$ ) und $10^{\circ}$ (ca. $1200 \mathrm{~nm}$ ) genommen (Abb.5.34 5.35 und 5.36). Bei niedrigen Energiedichten bis $3,6 \mathrm{~J} / \mathrm{cm}^{2}$ bildet sich

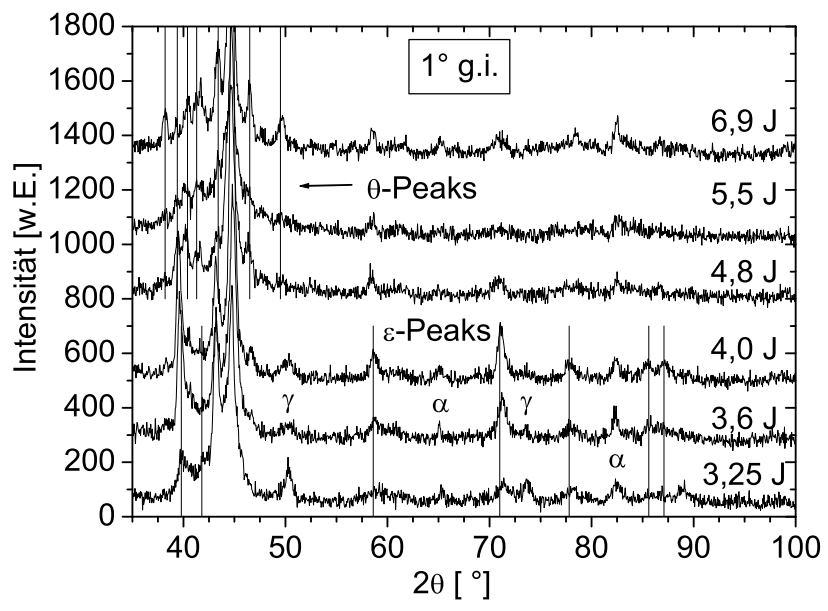

Abbildung 5.34: XRD-Spektren der Energie-Serie bei einem Einfallswinkel von $1^{\circ}$.

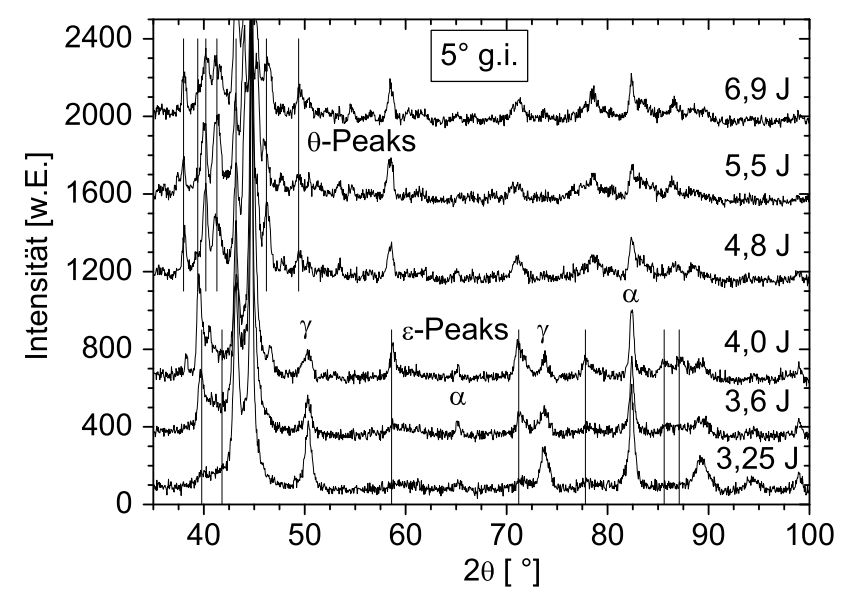

Abbildung 5.35: XRD-Spektren der Energie-Serie bei einem Einfallswinkel von $5^{\circ}$.

$\gamma-\mathrm{Fe}(\mathrm{C})$ mit einem geringen Anteil $\varepsilon$-Carbid, das ab $4 \mathrm{~J} / \mathrm{cm}^{2}$ bis $4,8 \mathrm{~J} / \mathrm{cm}^{2}$ ausschließlich vorhanden ist. Selbst bei $5,5 \mathrm{~J} / \mathrm{cm}^{2}$ und $6,9 \mathrm{~J} / \mathrm{cm}^{2}$ ist ein hoher Anteil $\varepsilon$-Carbid vorhanden. Bei diesen Energien bildet sich auch zunehmend $\theta$-Carbid. Vergleicht man die Phasenbildung bei einer bestimmten Energiedichte in 


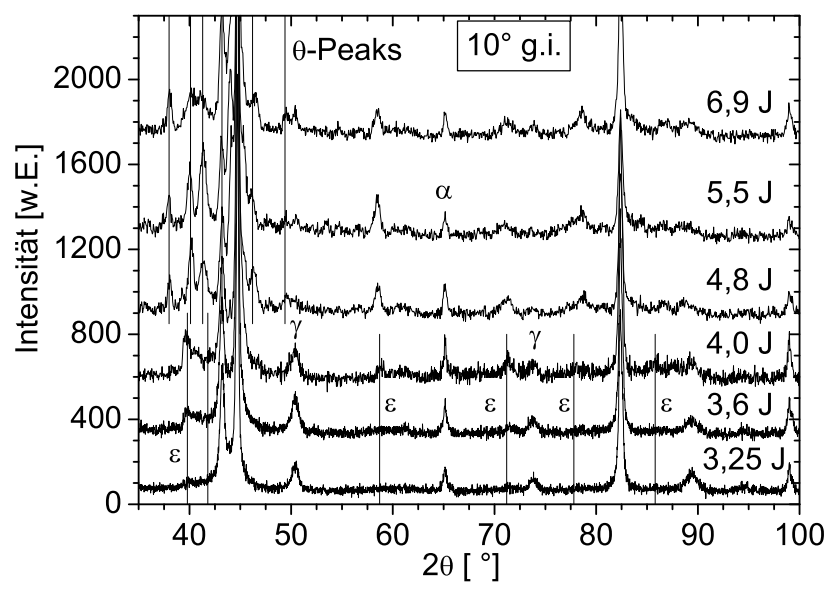

Abbildung 5.36: XRD-Spektren der Energie-Serie bei einem Einfallswinkel von $10^{\circ}$.

Abhängigkeit der Tiefe bzw. Einfallswinkel, so fällt auf, dass alle 3 XRD-Spektren die gleiche PhasenAbfolge aufweisen. D.h. die bei jeder Energiedichte gebildeten Phasen sind relativ gleichmäßig über die gesamte Tiefe bis ca. $1200 \mathrm{~nm}$ verteilt. In keinem Spektrum ist zu beobachten, dass z.B. bei gegebener Energiedichte an der Oberfläche und im Bulk verschiedene Phasen entstehen. Das inhomogene Profil des Roh-Strahls verursacht anscheinend starke laterale Druckgradienten auf der flüssigen Oberfläche, die letztlich zu Konvektionen und somit zur gleichmäßigen Durchmischung des inkorporierten Kohlenstoffs im Material führt.

Das CEM-Spektrum in Abb. 5.37 zeigt die charaktristischen breiten Unterspektren des $\varepsilon$-Carbides im Energiebereich ab $4 \mathrm{~J} / \mathrm{cm}^{2}$. Die entsprechende Flächenanteile der Phasen sind in Abb. 5.38 dargestellt Bei der Energieserie zeigt sich besonders, dass XRD- und CEM-Spektren für sich alleine keine eindeutige

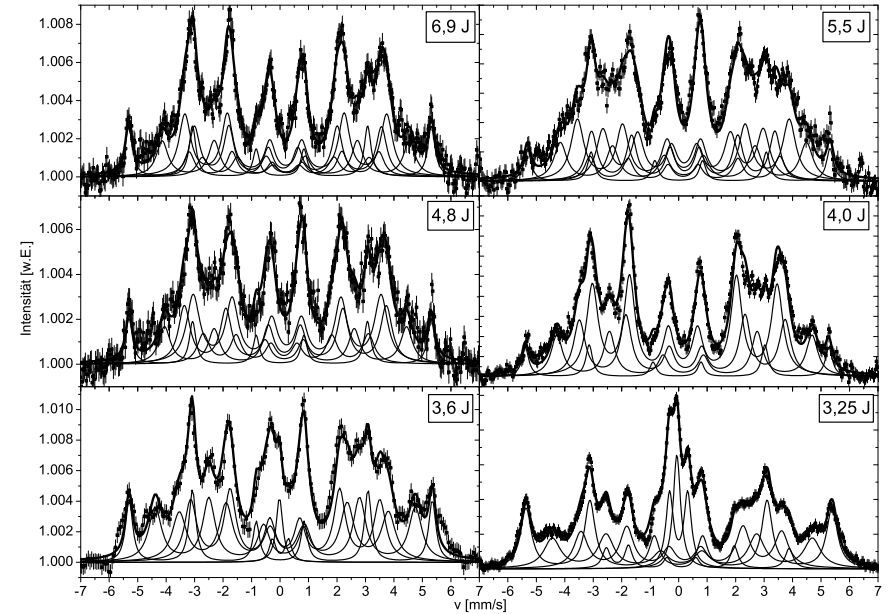

Abbildung 5.37: Mössbauerspektrum der Energieserie bei $\mathrm{p}\left(\mathrm{CH}_{4}\right)=0,1$ bar und $11 \times 12$ Pulsen.

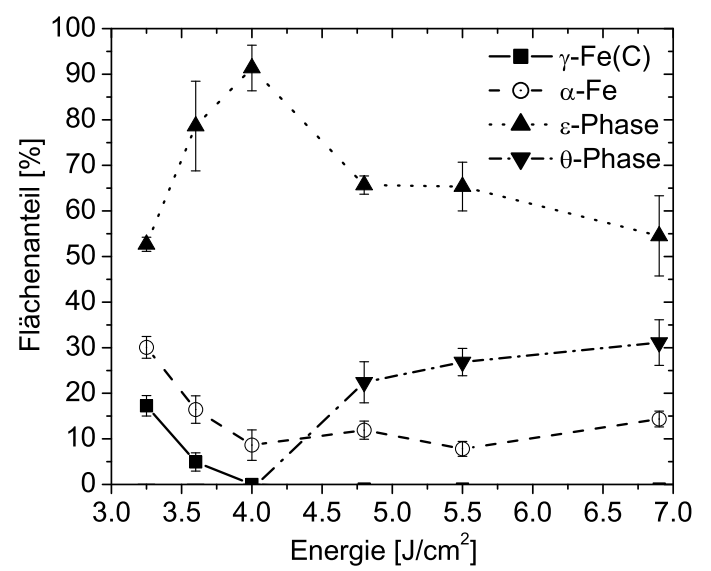

Abbildung 5.38: Flächenanteil der Fe-C-Phasen der Energieserie.

Aussagekraft besitzen:

- Die $\varepsilon$-Unterspektren des CEMS sind so breit, dass mögliche Spektren des $\theta$-Carbids verdeckt werden. Das CEMS deutet vorwiegend auf $\varepsilon$-Carbid hin.

- Das XRD-Spektrum zeigt einen erheblichen Anteil $\theta$-Carbid, das man besonders anhand des niedrigen $2 \theta$-Bereichs identifizieren kann.

Dass im CEMS auch $\theta$-Carbid-Unterspektren angepasst werden müssen, zeigt sich somit erst durch Auswertung der XRD-Reflexe.

Nur so war es auch möglich, das $\varepsilon$-Carbid anhand der PDF-Datenbank eindeutig zu identifizieren. Das korrespondierende CEM-Spektrum kann durch genau 3 Unterspektren angepasst werden, die jeweils 
zum $\mathrm{Fe}_{2} \mathrm{C}, \mathrm{Fe}_{3} \mathrm{C}$ und $\mathrm{Fe}_{6} \mathrm{C}$ gehören. Die so erhaltenden Hyperfeinparameter kann man zur Identifizierung des $\varepsilon$-Carbids heranziehen. Das CEM-Spektrum an sich hätte nicht zur Identifizierung des $\varepsilon$-Carbids ausgereicht, da die Literaturwerte von den hier erhaltenden Ergebnissen abweichen.

Bei der $\mathrm{p}\left(\mathrm{CH}_{4}\right)=0,1$ bar-Serie hat man gesehen, dass die Entstehung des Kohlenstoffs von dem SpotÜberlapp herrührt. Bei dieser Energieserie wird der Überlapp mit $11 \times 12$ Pulsen konstant gehalten, und die steigende Energiedichte ist hier für den Kohlenstoff verantwortlich.

Der Kohlenstoffniederschlag auf den Proben setzt sich zusammen aus Kohlenstoff, der fest mit der Oberfläche verbunden ist und dem losen, rußähnlichen Fallout. Betrachtet man die Energieserie von 3,25 bis $6,9 \mathrm{~J} / \mathrm{cm}^{2}$ aufwärts, so lässt sich der Kohlenstoff-Niederschlag folgendermaßen charakterisieren: Ab 3,6 $\mathrm{J} / \mathrm{cm}^{2}$ entsteht hellbrauner, fester Kohlenstoff. Mit steigender Energiedichte ändert sich die Farbe dieses Films über blauschwarz nach schwarz bei $6,9 \mathrm{~J} / \mathrm{cm}^{2}$. Ab $4 \mathrm{~J} / \mathrm{cm}^{2}$ fällt zusätzlich auch feiner, rußähnlicher Kohlenstoff nieder. Die Farbe des Films läßt sich mit zunehmender Schichtdicke erklären (s. auch RBSSpektrum in Abb. 5.33). Die Raman-Messungen in Abb.5.39 bestätigen, dass die dominierenden Bindung vom graphitischen $s p^{2}$-Typ ist 192 193 194]: Das Gesamtspektrum einer Messung ist aus 2 Einzelmessun-

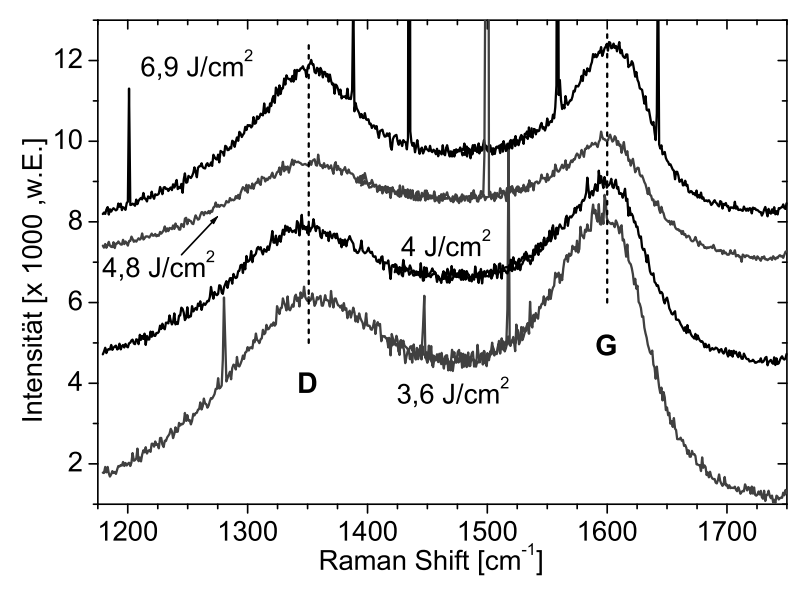

Abbildung 5.39: Ramanspektren der Energieserie. Die charakteristischen $D$ - und $G$-Peaks sind typisch für $s p^{2}$ gebunden Kohlenstoff. Die scharfen Peaks (spikes) sind Störungen, die im Analysator durch Höhenstrahlung verursacht werden. Die Spektren wurden jeweils aus 2 Einzelspektren zusammengesetzt, dadurch entsteht der Überlapp im Bereich um $1450 \mathrm{~cm}^{-1}$.

gen zusammengesetzt. Die den Spektren überlagerten Intensitätsspitzen kommen durch Höhenstrahlung im Analyse-System zustande, dessen Spektrometer aus einer CCD-Kamera besteht. Der D-Peak bei ca. $1350 \mathrm{~cm}^{-1}$ ist dem sog. breathing mode eines Kohlenstoff-6er-Ringes zuzuschreiben. Der $G$-Peak bei ca. $1600 \mathrm{~cm}^{-1}$ wird durch die relative Bewegung der $s p^{2}$-Bindungen verursacht. Das Auftreten der beiden $G$ - und $D$-Peaks sind ein klares Indiz für die Präsenz von nanokristallinem/amorphem Kohlenstoff [192] bzw. von kleinen graphitischen Kristalliten [194]. Die mehr qualitative Betrachtung soll nur untermauern, dass die Bindungseigenschaften des ausfallenden Kohlenstoffs unabhängig von der Energiedichte sind. Das zeigt sich insbesondere dadurch, dass sich die Peak-Positionen und das Intensitätsverhältnis $I(D) / I(G)$ nicht ändern.

\subsection{Pulsserien bei 4 bar Methan und Energiedichten von 2,5 $\mathrm{Jcm}^{-2}$ bzw. $3 \mathrm{Jcm}^{-2}$}

Desweiteren wurde untersucht, wie sich die Phasenbildung bei niedrigeren Energiedichten als $4 \mathrm{~J} / \mathrm{cm}^{2}$ vollzieht. Die niedrigste Energiedichte wurde mit $2,5 \mathrm{~J} / \mathrm{cm}^{2}$ so gewählt, dass die Temperatur $T$ gemäß Simulation (s. Abb. 4.1) gleich der Siedetemperatur $T_{s d}$ des Eisens ist. Nach der Theorie ist die Zündung des Plasmas notwendig, um auf der Oberfläche eine Reaktion herbeizuführen. Da die Energiedichte von $2,5 \mathrm{~J} / \mathrm{cm}^{2}$ offenbar gerade unterhalb der Schwelle liegt, die zur Metallverdampfung überschritten werden muss, ist keine Kohlenstoffaufnahme zu erwarten. Dieses Resultat wird auch mit dem CEM-Spektrum in Abb. 5.40 bestätigt: Selbst bei $16 \times 16$ Pulsen ist keine Änderung der Hyperfeinparameter festzustellen. Bei Bestrahlung bei dieser Energiedichte wird kein Kohlenstoff in das Material eingebracht, obwohl die 


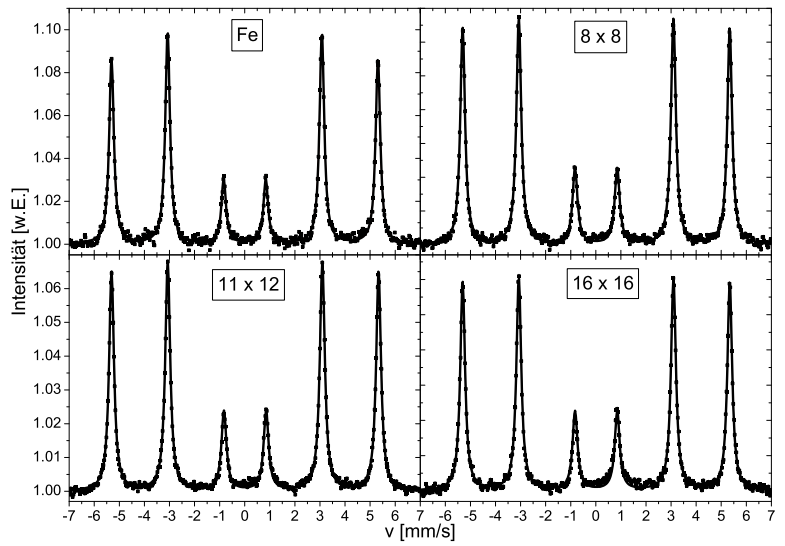

Abbildung 5.40: CEM-Spektren der Pulsserie bei 2,5 J/ $\mathrm{cm}^{2}$.

Oberfläche aufgeschmolzen ist. Das deutet darauf hin, dass die Dissoziation des Methans erst durch die Metallverdampfung bzw. die darauffolgende Plasmabildung initiiert wird. Die RBS-Spektren dieser Reihe sind identisch mit der Fe-Referenzprobe und sind daher nicht dargestellt. Eine Erhöhung der Energiedichte auf $3 \mathrm{~J} / \mathrm{cm}^{2}$ liegt über dieser Schwelle und führt daher zur Aufnahme des Kohlenstoffs. Es bildet sich

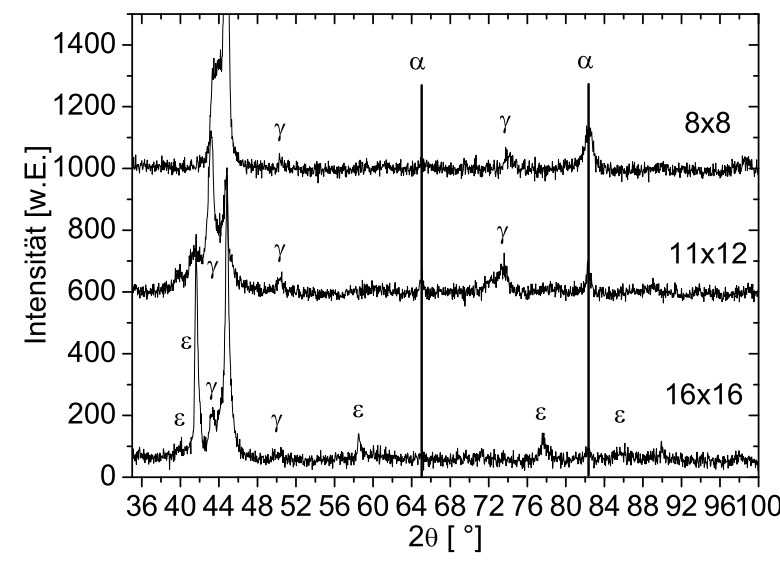

Abbildung 5.41: XRD-Spektren der Proben bei $\mathrm{p}\left(\mathrm{CH}_{4}\right)=4$ bar und $3 \mathrm{~J} / \mathrm{cm}^{2}$. Der Einfallswinkel beträgt $1^{\circ}$.

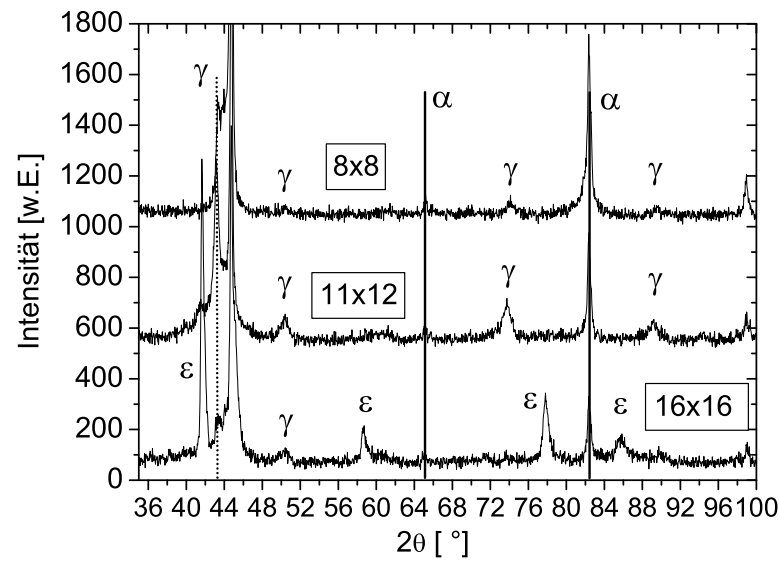

Abbildung 5.42: XRD-Spektren der Proben bei $\mathrm{p}\left(\mathrm{CH}_{4}\right)=4$ bar und $3 \mathrm{~J} / \mathrm{cm}^{2}$. Der Einfallswinkel beträgt $5^{\circ}$.

aber im Gegensatz zur Bestrahlung mit $4 \mathrm{~J} / \mathrm{cm}^{2}$ bei $8 \times 8$ kein $\alpha^{\prime}$-Fe, sondern $\gamma$-Fe(C) (s. Abb. 5.43 ) Interessanterweise entsteht kein $\theta$-, sondern das $\varepsilon$-Carbid, das eigentlich bei niedrigen Drücken auftritt.

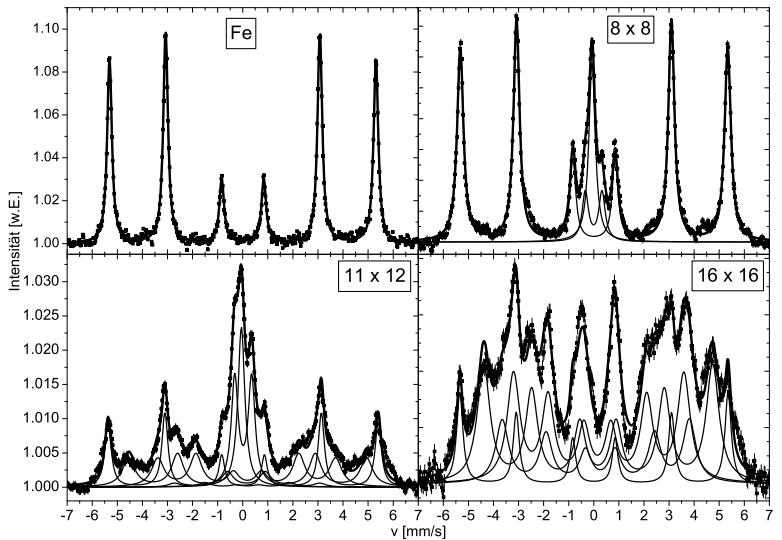

Abbildung 5.43: CEM-Spektrum der Pulsserie bei $3 \mathrm{~J} / \mathrm{cm}^{2}$.

Die XRD-Spektren in Abb. 5.41 und 5.42 zeigen klar die Entstehung des $\gamma$-Fe(C) und $\varepsilon$-Carbids. 


\subsection{Doppel-Bestrahlung}

Bei der Doppel-Bestrahlung wird die Probe bei einer Energiedichte von $4 \mathrm{~J} / \mathrm{cm}^{2}$ zweimal hintereinander auf derselben Fläche mit jeweils $8 \times 8$ Pulsen aber abwechselnd unter Hoch- und Nieder-Druck bestrahlt. Der Druck beträgt dabei 4 und 0,05-0,1 bar. Erwartungsgemäß ist die Probe, die im letzten Bestrahlungszyklus unter niedrigem $\mathrm{CH}_{4}$-Druck bestrahlt wird, auf der Oberfläche schwarz. Die Pulszahl beträgt somit $2 \times(8 \times$ $8)=128$, was ungefähr den üblichen $11 \times 12$ Pulsen entspricht. Von den anderen Serien ist bereits bekannt, dass sich bei $11 \times 12$ Pulsen und $\mathrm{p}\left(\mathrm{CH}_{4}\right)=4$ bar hauptsächlich $\theta$ - bzw. bei 0,1 bar $\varepsilon$-Carbid bildet. Um das $\theta$-Carbid zu bilden, bedarf es außer einer hohen Anzahl von Pulsen offensichtlich einen permanenten hohen Druck.

Weiterhin ist es überraschend, dass unabhängig von der Reihenfolge des angewandten Methandruckes $\varepsilon$-Carbid das Endprodukt ist. Bei $8 \times 8$ Pulsen und $\mathrm{p}\left(\mathrm{CH}_{4}\right)=0,1$ bar wird dieses Carbid bereits gebildet. Bei $\mathrm{p}\left(\mathrm{CH}_{4}\right)=4$ bar und $8 \times 8$ Pulsen wird $\alpha^{\prime}$-Fe beobachtet (deutlicher im XRD-Spektrum zu sehen.)

Die XRD-Spektren (Abb. 5.44) zeigen alle ausnahmslos die Formation des $\varepsilon$-Carbids und sind frei von jeglichen Reflexen des $\theta$-Carbids. Die entsprechenden CEM-Spektren setzen sich aus bekannten, typischen

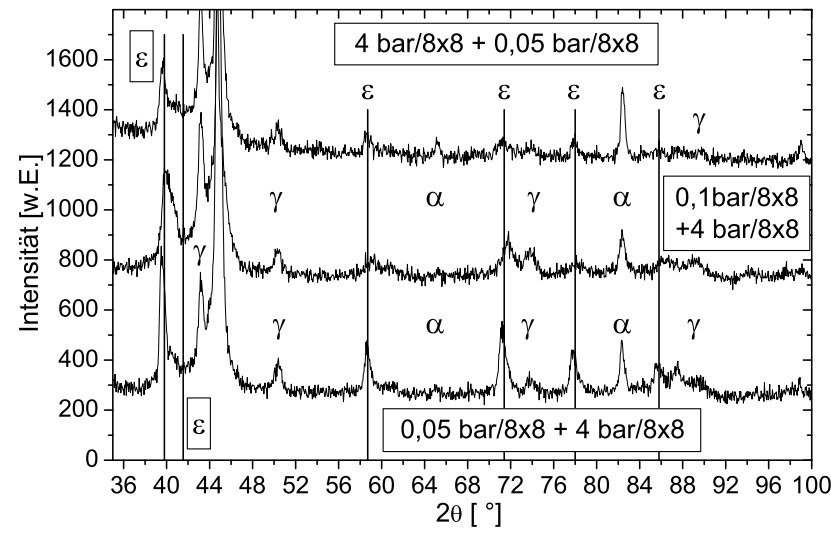

Abbildung 5.44: XRD-Spektren der Proben, die zweimal hintereinander mit $8 \times 8$ Pulsen, aber unter verschiedenen Drücken bestrahlt wurden.

breiten $\varepsilon$-Unterspektren wie in Abb. 5.45 zusammen. Die CEM-Spektren unterscheiden sich lediglich im

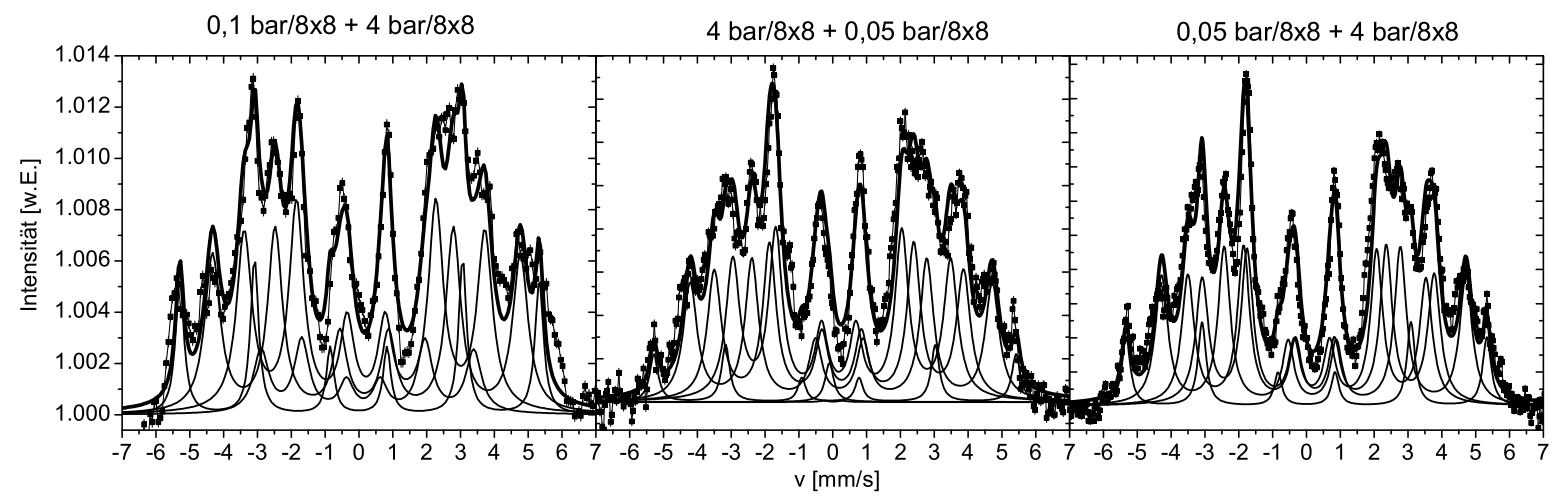

Abbildung 5.45: CEM-Spektren der doppelt-bestrahlten Proben.

unterschiedlichen Flächenanteil der Unterspektren, die verschiedenen Anteilen von $\mathrm{Fe}_{2} \mathrm{C}, \mathrm{Fe}_{3} \mathrm{C}$ und $\mathrm{Fe}_{6} \mathrm{C}$ zuzuordnen sind. Hier zeigt auch wie bei der $3 \mathrm{~J} / \mathrm{cm}^{2}$-Serie, dass $\varepsilon$-Carbid entsteht, ohne dass zwingend eine Kohlenstoffschicht mit der Carbid-Bildung einhergehen muss. Diese ist zweifelsfrei eine Folge des Druckes und nicht der Bildung des $\varepsilon$-Carbids. Hierbei ist anzumerken, dass der C-Gehalt im $\varepsilon$-Carbid in einem großen Bereich variieren kann, während im $\theta$-Carbid der Gehalt genau 25 at.\% betragen muss. 


\subsection{Thermo-Kopplungs-Messung}

Bei verschiedenen Drücken und Pulszahlen werden unterschiedliche Phasen gebildet, was sicherlich von verschiedener Kohlenstoffdiffusion und Temperaturverteilung bzw. -gradienten im Bulkmaterial zusammenhängt. Zur Erfassung der Wärmeleitung durch die Probe hindurch in Abhängigkeit von Druck und Pulszahl ( $8 \mathrm{~Hz}$ Wiederholfrequenz) wurde ein Thermo-Kopplungs-Experiment durchgeführt. Zu diesem Zweck wurde auf der Rückseite der $5 \times 5 \mathrm{~mm}^{2}$ großen Probe ein Eisen-Konstantan(45Ni55Cu)-Thermoelement angebracht und über einen dünnen PMMA-Steg auf dem Boden der Kammer befestigt. Die gemessenen Spannungen liegen im mV-Bereich. Um möglichst identische Kontaktierungsbedingungen zwischen Draht-

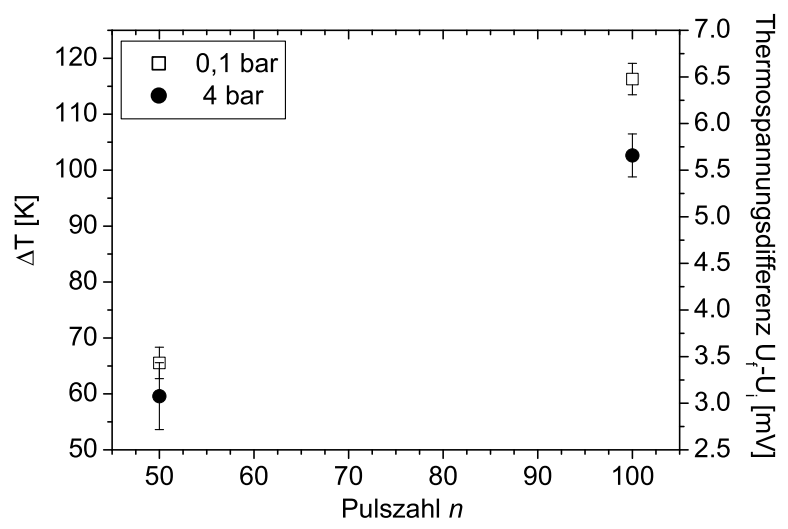

Abbildung 5.46: Gemessene Thermospannung/Temperatur in Abhänigkeit vom Druck und der Pulszahl.

Draht und Draht-Probe zu gewährleisten, wurde eine einzige Probe abwechselnd unter einem Druck von $\mathrm{p}\left(\mathrm{CH}_{4}\right)=0,1$ und 4 bar mit 50 bzw. 100 Einzelpulsen $(8 \mathrm{~Hz})$ bestrahlt. Die Messreihen wurden erst nach mehrmaligem, abwechselndem Bestrahlen aufgenommen, nachdem sich die Schwärze auch nicht mehr merklich geändert hatte und folglich die Reflektivität auf einen möglichst konstanten Wert gehalten wurde. Dadurch sollte errreicht werden, dass sich eine Art ,stationäres" Phasengemisch bildet, dessen Zusammensetzung sich beim Wechsel des Umgebungsdruckes nicht beträchtlich ändert, was natürlich nicht nachgeprüft werden konnte. Die Ursachen einer Veränderung der gemessenen Temperaturveränderung kann man somit etwas eingrenzen und dementsprechend Vorgängen auf der Oberfläche oder im Plasma zuschreiben. Jedoch konnte bei direkt aufeinander folgenden Messungen festgestellt werden, dass die Thermo-Spannungsdifferenz bei niedrigem Druck stets höher als bei hohem Druck bei gleicher Pulszahl (50 oder 100) ist (s. Abb. 5.46). Dies ist ein Hinweis darauf, dass der Gasdruck in der Kammer einen entscheidenden Einfluss auf das Plasma und die Energieeinkopplung in das Material ausübt. Die höhere Temperatur bei niedrigem Druck lässt darauf schließen, dass die Energieeinkopplung höher ist als bei hohem Druck, bei dem das dichtere Plasma die einfallende Laserstrahlung stärker absorbiert.

\subsection{Eigenspannungs-Bestimmung}

Gemäß der Gl. 3.90 beträgt die Steigung der Auftragung $\Delta d / d_{0}$ gegen $\sin ^{2} \Phi$ in Abb. 3.22 gerade das Verhältnis $(1+\nu) \sigma / E$. Negatives $\sigma$ bedeutet Druckspannung (compressive stress) und positives $\sigma$ Zugspannung (tensile stress) [108]. In Abb. 5.47 ist für die Probe, die mit $\mathrm{p}\left(\mathrm{CH}_{4}\right)=0,1$ bar bestrahlt wurde, das Verhältnis $(1+\nu) \sigma / E$ gegen die Anzahl der Pulse aufgetragen. Die beiden Einfallswinkel entsprechen den folgenden Tiefen: $1^{\circ} \approx 150 \mathrm{~nm}$ und $5^{\circ} \approx 750 \mathrm{~nm}$. Die Eigenspannung in den oberflächennahen Schichten ist größer als im Bulk. Mit steigender Pulszahl nimmt die negative Eigenspannung jedoch ab, weil mit steigendem Kohlenstoffgehalt die dadurch aufgebaute, ebenfalls steigende positive Eigenspannung entgegenwirkt. Der Anteil des Kohlenstoffs, der nur das $\varepsilon$-Carbid bildet (Abb. 5.49), wurde mit den CEMS-Unterspektren bestimmt (s. Kap. 5.13). Es konnten nur sehr wenige EigenspannungsBestimmungen durchgeführt werden, da bei der XRD-Analyse nur Reflexe benutzt werden können, die sehr schmal sind und zu einer Phase gehören. Die meisten Proben zeigen die Reflexe mehrerer Phasen und können aufgrund Überlappungen nicht präzise genug getrennt werden. Mit Erhöhung der Pulsanzahl 


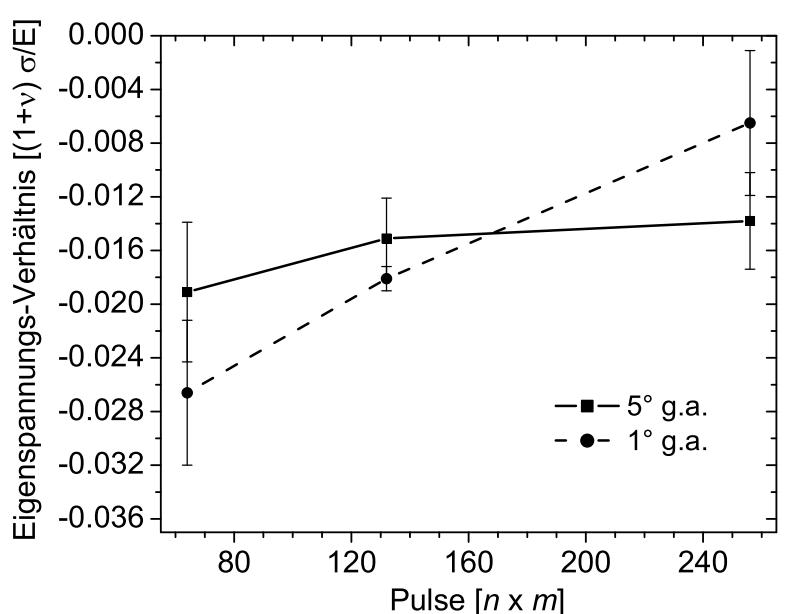

Abbildung 5.4\%: Tiefenabhängige Eigenspannung in den Proben der Pulsserie bei $\mathrm{p}\left(\mathrm{CH}_{4}\right)=0,1$ bar.

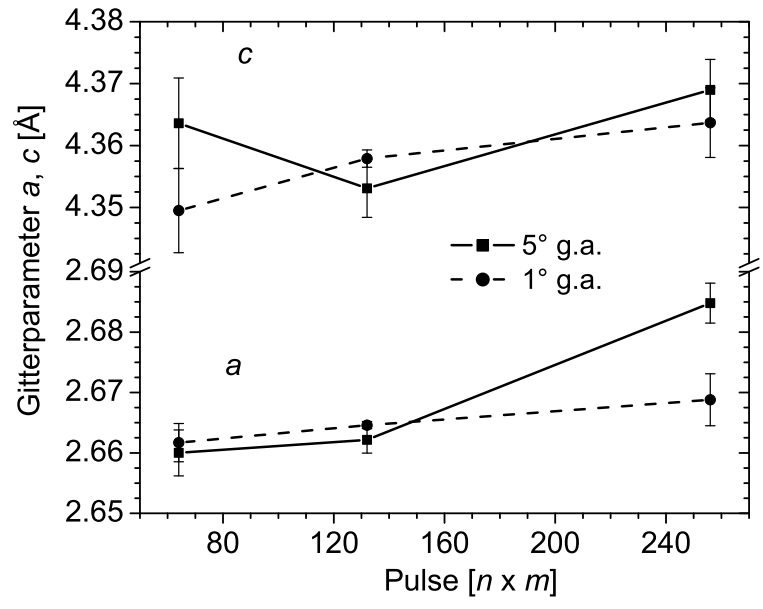

Abbildung 5.48: Die Änderung der Gitterparameter $a, c$ des $\varepsilon$-Carbids in Abhängigkeit der Pulsanzahl und somit des Kohlenstoffeintrags.

erhöht sich die Menge des inkorporierten Kohlenstoffs, was zu einer Vergrößerung der Gitterparameter $a$ und $c$ führt (s. Abb. 5.48).

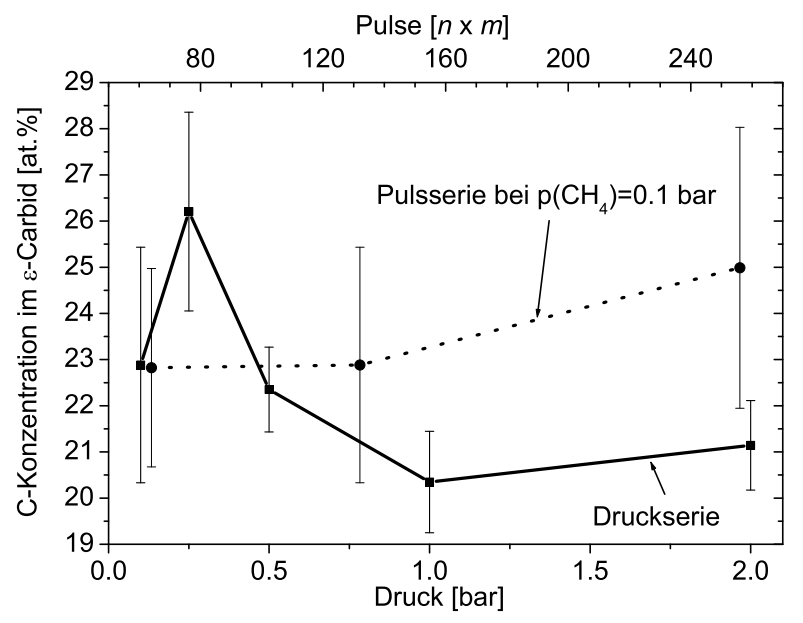

Abbildung 5.49: Der Kohlenstoffanteil des $\varepsilon$-Carbids in Abhängigkeit der Pulsanzahl und Druck.

\subsection{Kohlenstoffgehalt}

Multipliziert man den Flächenanteil der CEM-Spektren jeder Phase $A_{i}$ mit dessen Kohlenstoffanteil (in [at.\%]) $c_{i}$ und summmiert über alle Phasen, so erhält man den gesamten Kohlenstoffgehalt:

$$
c_{\text {tot }}[a t . \%]=\sum_{i} A_{i} \cdot c_{i}[a t . \%]
$$

Es kann jedoch nur der Kohlenstoff erfasst werden, der als Verbindung vorliegt und im CEM-Spektrum eindeutig identifiziert werden kann. Der Anteil des Kohlenstoffs $y_{C}$, der im fcc-Gitter des $\gamma$-Fe(C) eingelagert ist, errechnet sich zu [98]:

$$
x=\frac{A_{1}}{6 \cdot\left(A_{1}+A_{0}\right)} \quad \text { mit } \quad y_{C}=\frac{100 \cdot x}{1+x}
$$

mit: $A_{0}$ ist der Flächenanteil des Singuletts und $A_{1}$ der des Doubletts. Häufig überschreitet der erhaltende Wert $y_{C}$ den maximalen Löslichkeitswert von ca. 8,3 at.\%, der unter normalen Gleichgewichtsbedingungen 


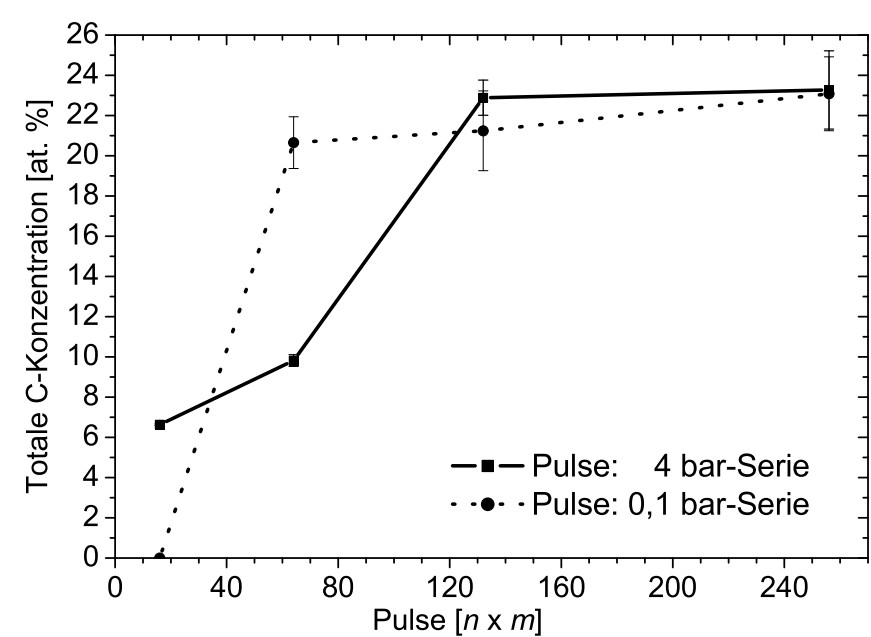

Abbildung 5.50: Gesamt-Kohlenstoffgehalt der Pulsserien bei $\mathrm{p}\left(\mathrm{CH}_{4}\right)=0,1$ bar und $\mathrm{p}\left(\mathrm{CH}_{4}\right)=4$ bar.

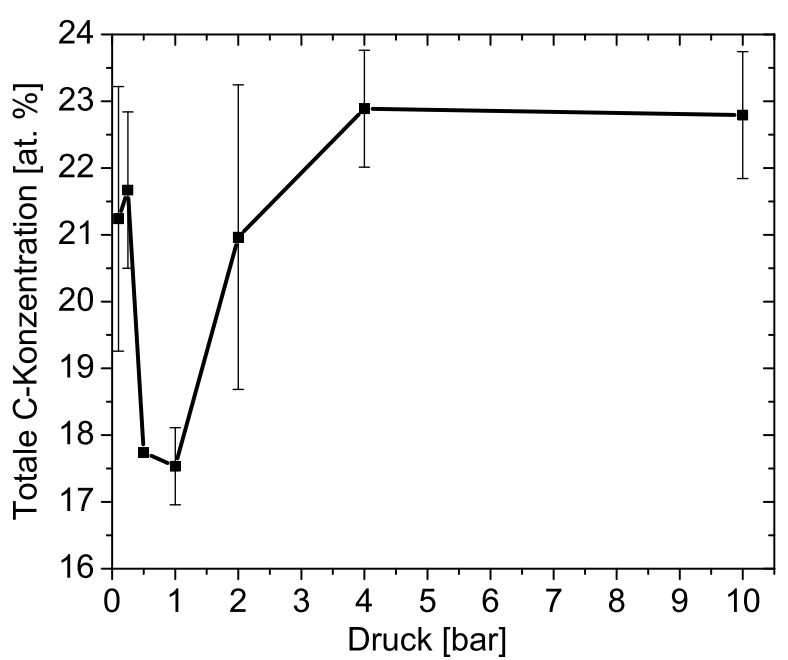

Abbildung 5.51: Gesamt-Kohlenstoffgehalt der Druckserie bei $11 \times 12$ Pulsen.

vorliegt. Aber aufgrund der hohen Drücke und Temperaturen bzw. deren Gradienten, die die Laserprozesse verursachen, entstehen auf diese Weise Nicht-Gleichgewichtsphasen mit enormen Kohlenstoffanteilen. Diese Beobachtung trifft besonders für die Edelstahlproben zu, die im nächsten Abschnitt diskutiert werden. In den Abb. 5.50 und 5.51 ist jeweils der Kohlenstoff-Gehalt der Pulsserien (bei $\mathrm{p}\left(\mathrm{CH}_{4}\right)=0,1$ bzw. 4 bar) und der Druckserie dargestellt. Durch Anfitten an die Funktion

$$
c(n, p)=c_{0} \cdot\left[1-\exp \left(-\frac{n-n_{0}}{t}\right)\right]
$$

wurde der maximale Kohlenstoffgehalt $c_{0}$ bestimmt. Dabei ist $n_{0}=0$ gesetzt, damit die $e$-Funktion durch $(0,0)$ geht, $t$ bestimmt die Form. In der Tab. 5.7 ist $c_{0}$ dieser Serien zusammengefasst und beträgt im Schnitt 25 at.\%. Der Kohlenstoffgehalt, den RBS-Messungen liefern, ist stets höher als der der CEM-

Tabelle 5.7: Maximaler Kohlenstoffgehalt (Sättigungsgehalt) $c_{0}$ für die Druck- und Pulsserien.

\begin{tabular}{|c||c|c|c|}
\hline $\begin{array}{c}\text { Kohlenstoff- } \\
\text { gehalt }\end{array}$ & $\begin{array}{c}\text { Druck- } \\
\text { Serie }\end{array}$ & $\begin{array}{c}\text { Pulsserie } \\
\mathrm{p}\left(\mathrm{CH}_{4}\right)=0,1 \text { bar }\end{array}$ & $\begin{array}{c}\text { Pulsserie } \\
\mathrm{p}\left(\mathrm{CH}_{4}\right)=4 \text { bar }\end{array}$ \\
\hline$c_{0}[$ at.\%] & $22,7(2)$ & $24,0(51)$ & $25,7(45)$ \\
\hline
\end{tabular}

Spektren, weil

a) auch Kohlenstoff detektiert wird, der elementar vorliegt

b) unbekannte oder nicht eindeutig aufösbare Unterspektren autreten können, denen kein KohlenstoffAnteil zugeordnet werden kann. Diese Spektren liegen beispielsweise zwischen zwei scharfen Peaks oder machen sich als Schultern bemerkbar, wie es in Abb. 5.28 der Fall ist.

\subsection{Mikrohärte-Messungen}

Die mechanischen Eigenschaften wurden mit dem Nanoindenter untersucht. Es wurden jeweils mindestens 20 Messungen an der Probe vorgenommen und gemittelt. Ausreißer wurden nicht in die Mittelung einbezogen. Dass manchmal recht abweichende Härtekurven aufgenommen wurden, liegt wahrscheinlich an den Resultaten der Bestrahlungsweise. Das Mäandrieren hinterlässt auf der Oberfläche ein entsprechendes, aber sehr schwaches Muster (hervorgerufen durch den Piston-Effekt). Diese Inhomogenitäten können scheinbar untereinander verschiedene Härten aufweisen. 
Nach dem Modell von Jönsson und Hogmark [195] kann man die Härte $H$ einer Probe in Abhängigkeit der indentierten Tiefe $d$ abschätzen. Die Funktion lautet:

$$
H(d)=H_{s}+\left(H_{c}-H_{s}\right) \cdot\left[2 k \frac{t}{d}-k^{2}\left(\frac{t}{d}\right)^{2}\right]
$$

$H_{s}$ ist die Substrat- und $H_{c}$ die Oberflächenschicht der Dicke $t ; d$ ist die Indenter-Tiefe. Der Parameter $k$ beschreibt die plastische Deformation der indentierten Schicht und variiert zwischen $0,073<k<0,14$, wird jedoch anfangs der Konsistenz halber auf $\mathrm{k}=0,1$ fixiert. Damit das Modell gültig ist, muss das Verhältnis $d / t>0,1$ sein [24]. Da $t$ in der Größenordnung der Schmelztiefe von ca. $1 \mu$ m liegen sollte, ist das Modell für Indenter-Tiefen ab $d>100 \mathrm{~nm}$ anwendbar. Die Abb. 5.52 zeigt die Mikrohärte-Kurven für

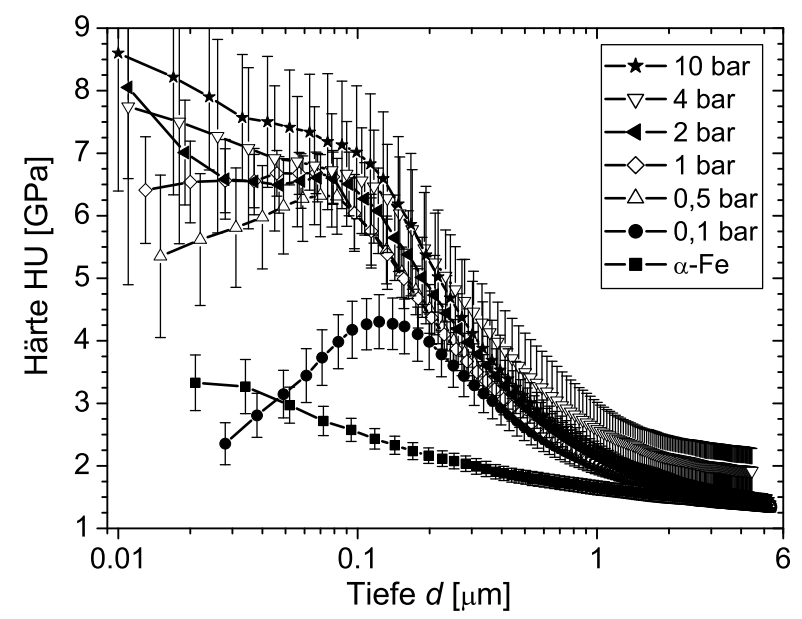

Abbildung 5.52: Mikrohärte-Kurven der Proben bei $11 \times 12$ Pulsen und variablen Methandrücken. Mit steigendem $\theta-\mathrm{Fe}_{3} \mathrm{C}-\mathrm{Gehalt}$ nimmt die Härte zu.

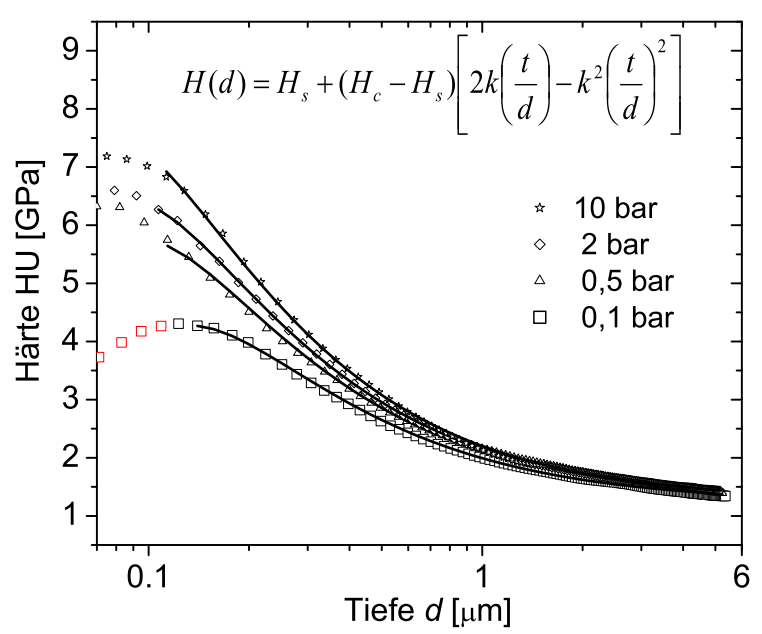

Abbildung 5.53: Mikrohärte-Kurven aus Abb. 5.52, die mit der Formel (5.6) angepasst wurden.

die Druckserie bei $11 \times 12$ Pulsen. Das Ergebnis deckt sich mit den XRD- und CEMS-Messungen, dass bei steigendem Druck der Anteil an hartem $\theta-\mathrm{Fe}_{3} \mathrm{C}$ wächst, was auch die Fits in Tab. 5.8 übereinstimmend wiedergeben. Die Mikrohärtekurven (Abb. 5.52) wurden mittels der Gl. (5.6) angefittet und in sind in Abb. 5.53. In der Tabelle 5.8 sind die angepassten Parameter aufgelistet. Die Eisen(Substrat-)härte

Tabelle 5.8: Härtewerte der Druckserie bei $11 \times 12$ Pulsen.

\begin{tabular}{|c||c|c|c|c|}
\hline Druck [bar] & $k$ & $H_{s}[\mathrm{MPa}]$ & $H_{c}[\mathrm{MPa}]$ & $t[\mu \mathrm{m}]$ \\
\hline \hline \multirow{2}{*}{0,1} & 0,14 & $1229(3)$ & $4332(11)$ & $0,94(1)$ \\
& 0,1 & $1226(3)$ & $4297(13)$ & $1,34(1))$ \\
\hline 0,5 & 0,1 & $1291(5)$ & $5735(44)$ & $0,98(1)$ \\
\hline 2 & 0,1 & $1274(1)$ & $6397(13)$ & $0,90(<0,01)$ \\
\hline \multirow{2}{*}{10} & $0,085(1)$ & $1247(4)$ & $7441(58)$ & 0,94 \\
& 0,1 & $1247(4)$ & $7441(58)$ & $0,80(1)$ \\
\hline
\end{tabular}

beläuft sich im Mittel auf 1260(24) MPa bei einer mittleren Schichtdicke von 0,9 $\mu \mathrm{m}$, was sehr gut zu der simulierten Aufschmelztiefe von ca. $950 \mathrm{~nm}$ bei $4 \mathrm{~J} / \mathrm{cm}^{2}$ (Abb. 4.2 passt. Zu beachten ist die Dicke der 0,1 bar-Probe, die mit $t=1,35 \mu \mathrm{m}$ die Bedingung $d / t>0,1$ für $k=0,1$ nicht über die gesamte Indenter-Tiefe $d$ erfüllt, bei $k=0,14$ jedoch den erwarteten Wert $t=0,94 \mu \mathrm{m}$ erreicht. Andererseits ist für die Probe bei $\mathrm{p}\left(\mathrm{CH}_{4}\right)=10$ bar die Tiefe mit $t=0,8 \mu \mathrm{m}$ etwas zu gering. Bei einem Wert von $k=0,085$ ergibt der Fit die Tiefe der 0,1 bar-Probe. Die plastische Deformierbarkeit der Oberfläche ändert sich also sehr stark, was wegen der Kohlenstoffschicht im Vergleich zu Zementit zu erwarten ist.

Die Härten der Pulsserien bei $\mathrm{p}\left(\mathrm{CH}_{4}\right)=0,1$ und 4 bar sind in den Abb. 5.54 und Abb. 5.55 dargestellt. Die Härtekurven bei $\mathrm{p}\left(\mathrm{CH}_{4}\right)=0,1$ bar weisen außer bei $4 \times 4$ Pulsen ein Maximum auf. Dieses Maximum 


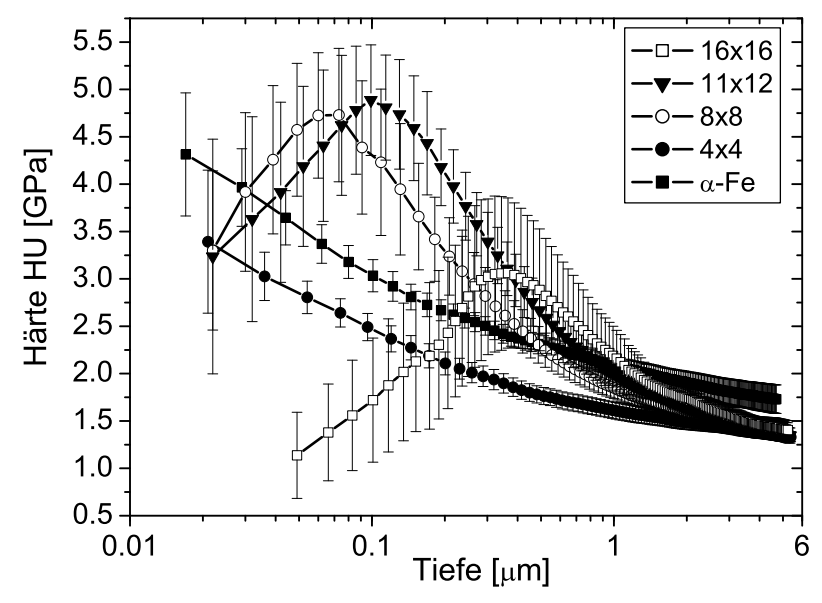

Abbildung 5.54: Mikrohärte-Kurven der Proben bei $\mathrm{p}\left(\mathrm{CH}_{4}\right)=0,1$ bar.

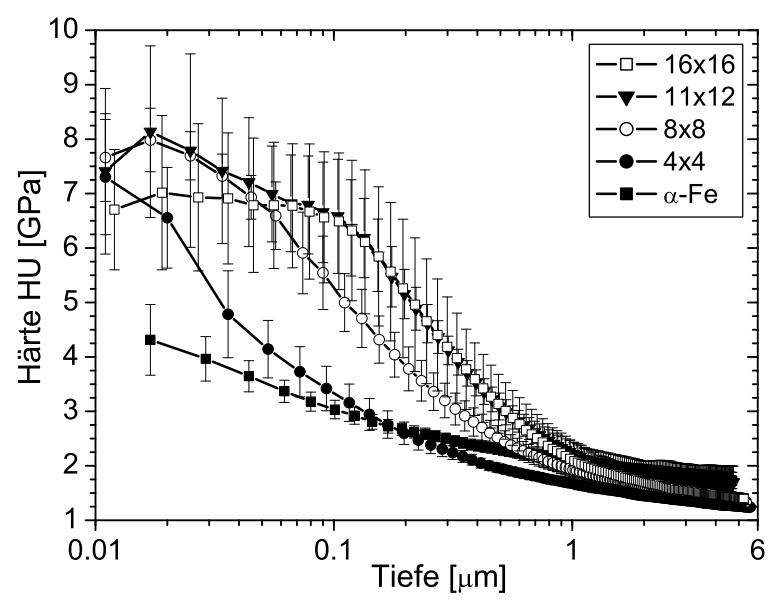

Abbildung 5.55: Mikrohärte-Kurven der Proben bei $\mathrm{p}\left(\mathrm{CH}_{4}\right)=4$ bar.

verschiebt sich mit zunehmender Pulszahl zu größeren Tiefen hin, da die Kohlenstoffschicht entsprechend ansteigt. Der Nanoindenter misst die Matrix aus Carbid und weichem Kohlenstoff, dessen Anteil mit zunehmender Tiefe abnimmt. Die Proben der $\mathrm{p}\left(\mathrm{CH}_{4}\right)=4$ bar-Serie weisen erwartungsgemäß die Tendenz auf, dass mit steigender Pulszahl die harte $\theta$-Carbid-Schicht dicker wird. Die $8 \times 8$-Probe weist eine ähnliche Härte auf. Diese ist gemäß CEMS-Messung auf das ebenfalls harte und spröde Martensit zurückzuführen. In Abb. 5.56 ist die Härte über den CEMS-Flächenanteil des $\theta$-Carbids der Druckserie aufgetragen. Dabei

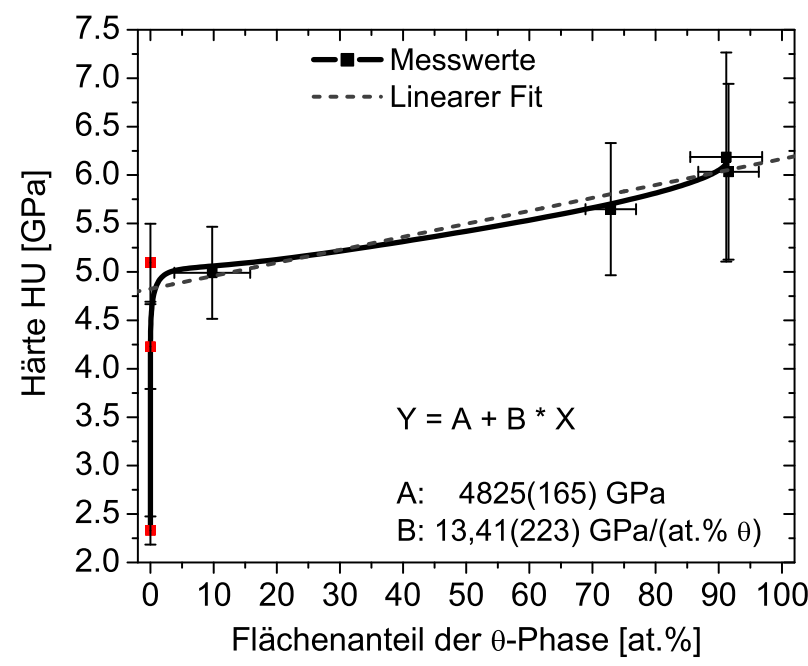

Abbildung 5.56: Abhängigkeit der Gesamthärte vom Gehalt des $\theta$-Carbids. Der Härte-Beitrag der anderen Phasen ist nicht berücksichtigt. Dennoch gilt der lineare Zusammenhang für die Teilphasen.

wurde der Härtewert HU in $150 \mathrm{~nm}$ Tiefe genommen, was auch der CEMS-Informationstiefe entspricht. Die experimentellen Werte können gut mit einem linearen Fit angepasst werden. Die Härte steigt linear mit dem Gehalt der $\theta$-Phase an. Es ist jedoch zu bemerken, dass hier die Gesamthärte in Abhängigkeit des Anteils einer Teilphase aufgetragen ist. Zu der Gesamthärte tragen natürlich auch die nicht berücksichtigten Phasen $\varepsilon$-Carbid, $\alpha$-Fe und $\gamma$-Fe(C) bei. Für die Gesamthärte $H_{\text {ges }}$ eines 2-Phasen-Gefüges gilt beispielsweise einfach die Summenregel [16]:

$$
H_{\text {ges }}=f_{1} \cdot H_{1}+f_{2} \cdot H_{2} \quad, \quad \text { mit } f_{1}+f_{2}=1,
$$

dabei ist $f_{i}$ der Volumenbruch der Komponente $H_{i}$. Bei mehreren Komponenten ist diese Formel entsprechend zu erweitern:

$$
H_{\text {ges }}=\sum_{i} f_{i} \cdot H_{i} \quad, \quad \text { mit } \sum_{i} f_{i}=1
$$




\subsection{Zusammenfassung: Phasengebiete der Puls- und Druckserie}

Die Prozess-Parameter Druck und Pulszahl haben einen relativ geringen Spielraum bei der festen Energiedichte von $H=4 \mathrm{~J} / \mathrm{cm}^{2}$, um die einzelnen Phasen $\gamma$-Fe(C), $\alpha$-Fe, $\varepsilon$ - und $\theta$-Carbid zu erzeugen. Darüber hinaus gibt es auch mehrere Parameter-,,Kombinationen“, bei denen annähernd gleiche Resulte erzielt werden können. Der Flächenanteil der Phasen (außer $\alpha^{\prime}$-Fe) ist in Abhängigkeit von Druck und Pulsanzahl als 3D-Darstellung in Abb. 5.57 aufgetragen, um die Phasenbildungs-Gebiete veranschaulichen zu können. Die Energieabhängigkeit ist dabei in der Darstellung nicht berücksichtigt, da nur eine Serie bei

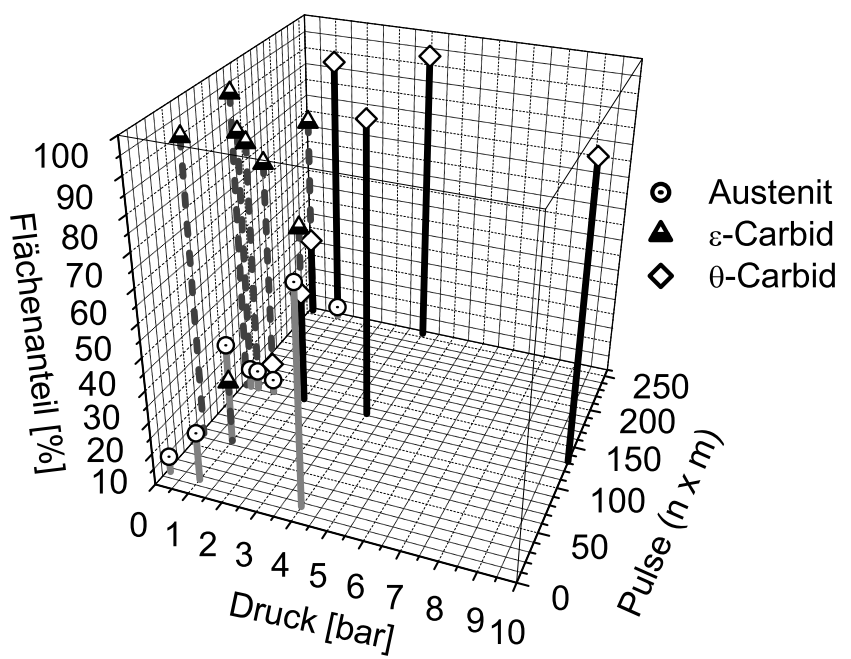

Abbildung 5.57: 3D-Ansicht des Flächenanteils in Abhängigkeit von Puls und Druck.

einem festem Druck $\left(\mathrm{p}\left(\mathrm{CH}_{4}\right)=0,1\right.$ bar $)$ und einer festen Pulszahl $(11 \times 12)$ durchgeführt wurde. 


\section{Kapitel 6}

\section{Ergebnisse der Edelstahl-Bestrahlung}

Der verwendete Edelstahl X5CrNiMo18.10.3 besitzt im Gegensatz zu $\alpha$-Eisen (bcc) eine kubisch-flächenzentrierte Struktur (fcc). Der austenitische Edelstahl wird analog dem Austenit $\gamma$ - $\mathrm{Fe}(\mathrm{C})$ mit $\gamma-\mathrm{Fe}(\mathrm{Cr}, \mathrm{Ni}, \mathrm{Mo})$ bezeichnet und soll andeuten, dass in dem fcc-Gitter außer Fe noch die substitutionellen Elemente Cr, Ni und Mo enthalten sind.

Die wichtigsten Mess-Parameter sind:

- XRD: Gitterparameter : $a=0,35946(10) \mathrm{nm}$. Die Gitterparameter wurden mithilfe der XRD-Reflexe einer unpolierten und unbestrahlten Probe berechnet. Die Aufnahme des Kohlenstoffs verursacht eine Vergrößerung der Gitterparameter $a_{0} \rightarrow a$. Kennt man die Gitterkonstanten aus den XRDSpektren, kann man umgekehrt die Kohlenstoff-Konzentration errechnen:

$$
a=a_{0}+0,00078(a t . \% C)
$$

Für $a_{0}=0,3572 \mathrm{~nm}$ ist das die bekannte Formel für $\gamma$-Fe(C) [9] und es wird hier davon ausgegegangen, dass sich der lineare Term für Edelstahl nur verschwindend gering ändert. $a_{0}$ ist die Gitterkonstante des kohlenstofffreien Grund- oder Ausgangsaustenits (austenite), a die Gitterkonstante des durch Kohlenstoffaufnahme gedehnten Austenits, des sog. expanded austenite.

- Mössbauer: Das CEM-Spektrum der unbehandelten und unpolierten Probe ist in Abb. 6.1 dargestellt. Die typischen Hyperfein-Parameter Isomerieverschiebung IS und Quadrupolaufspaltung $Q S$ sind in Tab. 6.1 aufgelistet:

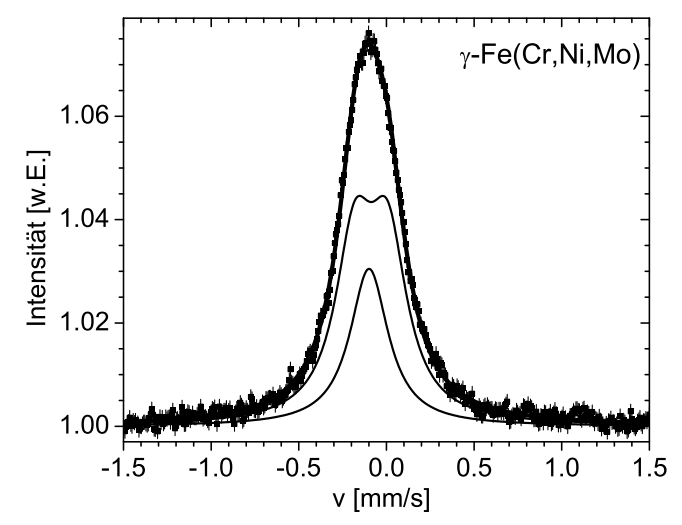

Tabelle 6.1: Richtwerte der Mössbauerparameter des austenitischen Edelstahls [196 197] (s. Abb.6.1). Der * bedeutet, dass diese Parameter beim Anpassen verknüpft wurden. Die Werte des Singuletts und des 1. Doubletts sind die Ausgangswerte des $\gamma-\mathrm{Fe}(\mathrm{C})$. NN bedeutet nächster Nachbar.

\begin{tabular}{|c||c|c|c|}
\hline & IS $[\mathrm{mm} / \mathrm{s}]$ & QS $[\mathrm{mm} / \mathrm{s}]$ & Ursache \\
\hline \hline Singulett & $\approx-0,07^{*}$ & - & kein C-NN \\
\hline 1. Doublett & $\approx 0,02$ & $0,65(2)$ & $1 \mathrm{C}-\mathrm{NN}$ \\
\hline 2. Doublett & $\approx-0,07^{*}$ & $0,18(1)$ & $\begin{array}{c}\text { kein C-NN } \\
\gamma-\mathrm{Fe}(\mathrm{Cr}, \mathrm{Ni}, \mathrm{Mo})\end{array}$ \\
\hline
\end{tabular}

Abbildung 6.1: CEM-Spektrum der unpolierten und unbehandelten Edelstahl-Probe.

Im Gegensatz zum $\gamma$-Fe(C) erscheint im CEM-Spektrum des $\gamma$-Fe(Cr,Ni,Mo) ein weiteres Doublett, das auch bei Abwesenheit des Kohlenstoffs im Gitter auftritt. Die substitutionellen Legierungselemente Cr, $\mathrm{Ni}$ und Mo verursachen durch die geringfügige Verzerrung des kubischen Symmetrie eine kleine Quadrupolaufspaltung. 


\subsection{Pulsserie bei 1 bar Methan}

Die Bestrahlung des Edelstahls X5CrNiMo18.10.3 führt zu gänzlich anderen Ergebnissen. Die Serie wurde bei einem Druck von $\mathrm{p}\left(\mathrm{CH}_{4}\right)=1$ bar von $2 \times 2$ bis $16 \times 16$ Pulsen bestrahlt. Das XRD-Spektrum in Abb.6.2 zeigt eine interessante Phasenentwicklung im Edelstahl. Ab $8 \times 8$ Pulsen entsteht allmählich eine amorphe

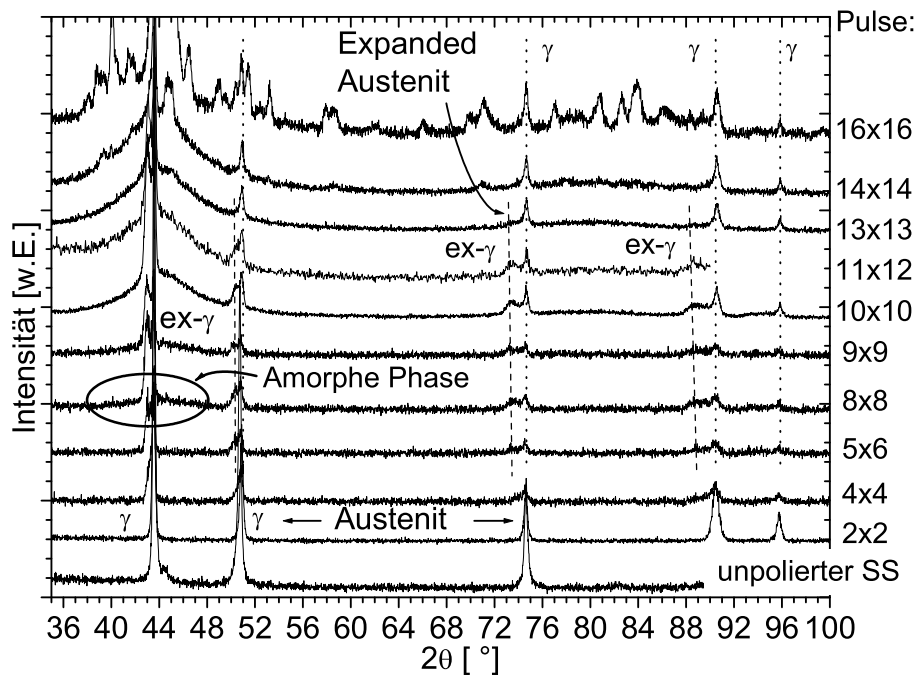

Abbildung 6.2: XRD-Spektren der Pulsserie bei $\mathrm{p}\left(\mathrm{CH}_{4}\right)=1$ bar.

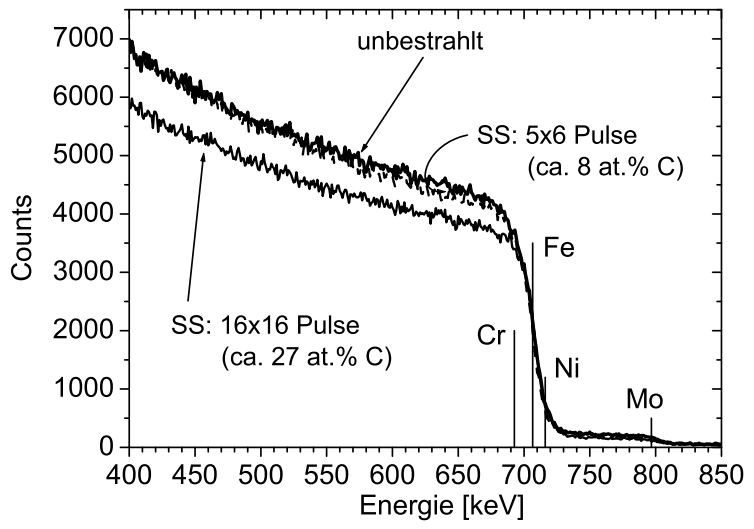

Abbildung 6.3: RBS-Spektrum der Pulsserie; der Übersichtlichkeit sind nur ausgewählte Proben dargestellt. Die maximale Kohlenstoffkonzentration beträgt ca. 27 at.\%, die Elemente geben die Positionen der Kanten an.

Phase, die an dem breiten Untergrund im Bereich von $2 \theta=40^{\circ}-47^{\circ} \mathrm{zu}$ sehen ist. Weiterhin tauchen zusätzlich zu den gewöhnlichen $\gamma$-Peaks des Grund-Austenits ab ca. $4 \times 4$ Pulsen zur low-angle-Seite weitere Peaks auf, die dem expanded austenite, $\gamma-\mathrm{Fe}(\mathrm{C})$ zuzuordnen sind und deren Abstand zueinander mit wachsender Pulszahl zunimmt. Es wurden auch entsprechende Messungen unter einem Einfallswinkel von $1^{\circ}(\approx 150 \mathrm{~nm})$ durchgeführt. Die Spektren ähneln der Form nach den Messungen unter $5^{\circ}$, sind jedoch aufgrund geringer Statistik und Untergrund sehr verrauscht. Diese zeigen ebenfalls den amorphen Untergrund und die Präsenz der $\gamma$-Fe(C)-(Doppel)Peaks, was auch durch die CEM-Spektren bestätigt wird (ungefähr gleiche Informationstiefe). Das Eigenspannungs-Verhältnis $\sigma / E$ in Abhängigkeit von der Pul-

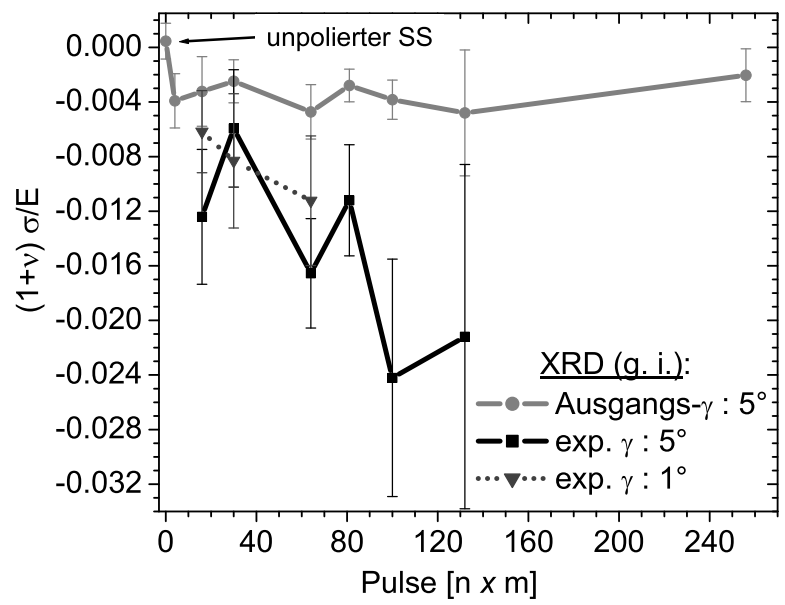

Abbildung 6.4: Eigenspannungs-Verhältnis $(1+\nu) \sigma / E$ in Abhängigkeit von der Pulszahl für expanded und Grund$\gamma-\mathrm{Fe}(\mathrm{C})$. Die Informationstiefen betragen ca. $150 \mathrm{~nm}\left(1^{\circ}\right.$ g.i. $)$ und $750 \mathrm{~nm}\left(5^{\circ}\right.$ g.i. $)$.

sanzahl wurde wie beim Eisen mit $\sin ^{2} \Phi$-Fits bestimmt. Die Abb. 6.4 zeigt die Eigenspannung des Grundund des expanded $\gamma-\mathrm{Fe}(\mathrm{C})$. Die Eigenspannung des fcc-Ausgangsgitters (austenite) ist nahezu konstant, während die Eigenspannung des C-inkorporierten fcc-Untergitters (expanded austenite) deutlich höher ist und mit der Pulsanzahl und somit dem C-Gehalt (im Rahmen der Messgenauigkeit) zunimmt. Das Vorzeichen von $\sigma$ ist negativ, somit liegt Druckspannung vor. Der Eigenspannungs-Wert des unpolierten 
Edelstahls liegt bei $(1+\nu) \sigma / E=0,00046(131)$, erwartungsgemäß der niedrigste Wert für eine unbehandelte Probe. Anders als beim $\varepsilon$-Carbid im Fe (Abb. 5.47) nimmt das Verhältnis $(1+\nu) \sigma / E$ beim Edelstahl mit der Pulszahl zu. Zum einen können die unterschiedliche kristallographische Struktur zum anderen die Beeinflussung anderer Phasen dafür verantwortlich sein.

Der Untergrund ist bei $10 \times 10$ Pulsen richtig ausgeprägt und bleibt bis $14 \times 14$ Pulsen ungefähr konstant. Bei $16 \times 16$ Pulsen verschwindet dieser Untergrund und eine Vielzahl von Reflexen tritt auf, die von unterschiedlichen, kristallinen Carbiden und $\gamma-\mathrm{Fe}(\mathrm{C})$ herrühren und schon etwas bei $14 \times 14$ zu erkennen sind. Das RBS-Spektrum (Abb. 6.3) deutet wie beim Eisen auf einen homogenen Kohlenstoffeintrag von maximal 27 at.\% hin, der jedoch grundlegend unterschiedliche Fe-C-Verbindungen und Phasen bildet. In

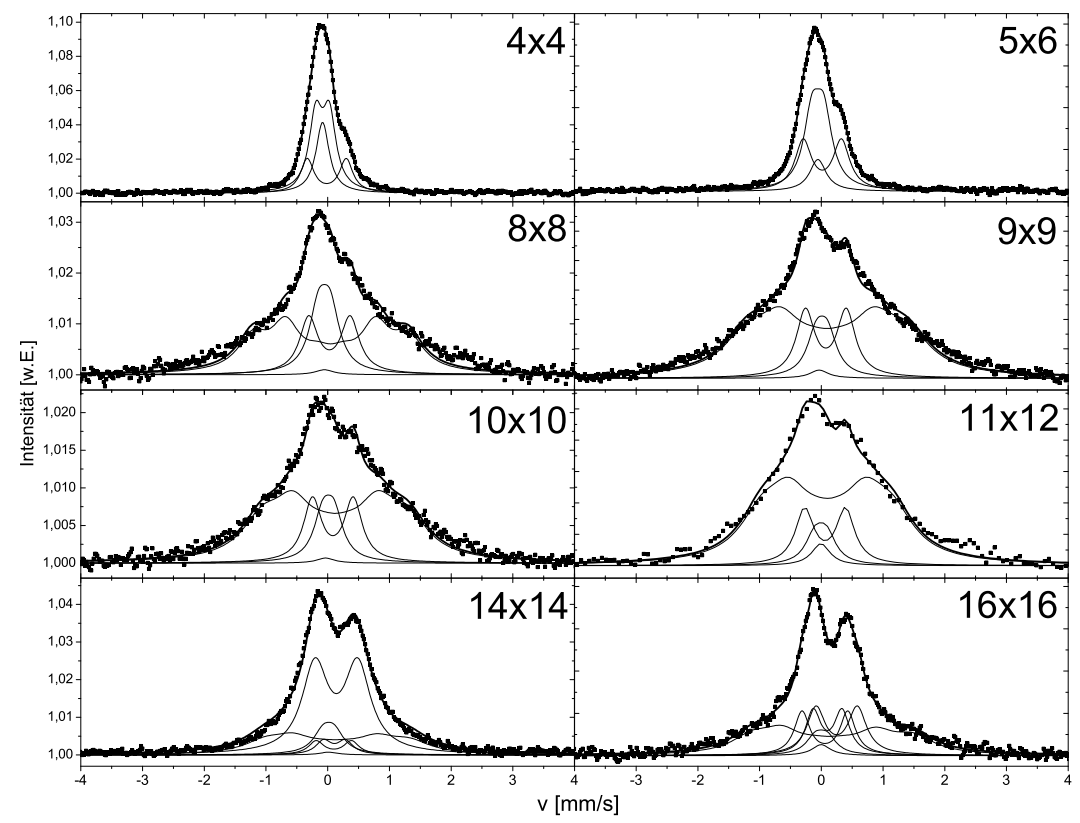

Abbildung 6.5: CEM-Spektren der Edelstahl-Pulsserie bei $\mathrm{p}\left(\mathrm{CH}_{4}\right)=1$ bar.

Abb. 6.6 ist der Kohlenstoffgehalt zusammengestellt, der sich aus den RBS-, XRD- und CEMS-Messungen ergibt. Dabei ist zu beachten, dass RBS den gesamten Kohlenstoff in der Probe detektiert und dass die Informationstiefe des CEMS mit $150 \mathrm{~nm}$ ca. ein Fünftel der des XRD-Spektrums mit $750 \mathrm{~nm}$ beträgt. Die XRD-Messungen wurden unter verschiedenen Einfallswinkeln durchgeführt und liefern deshalb Informationen aus verschiedenen Tiefen. Die Messungen zeigen aber dennoch, dass sowohl beide XRD-Spektren als auch das CEM-Spektrum ungefähr den gleichen Kohlenstoff-Anteil im $\gamma-\mathrm{Fe}(\mathrm{C})$. Der Kohlenstoffgehalt des $\gamma-\mathrm{Fe}(\mathrm{C})$ ist viel geringer als der Gesamtgehalt. Das CEM-Spektrum (Abb. 6.5) zeigt bis $5 \times 6$ Pulsen nur das Singulett bzw. Doublett des $\gamma$-Fe(C). Ab $8 \times 8$ Pulsen tritt ein breites, nicht weiter aufösbares Sextett auf, das sich in Übereinstimmung mit dem XRD-Spektrum (Abb. 6.2 als eine amorphe Phase herausstellt. Im Gegensatz zum XRD-Spektrum, in dem der amorphe Untergrund allmählich ansteigt, taucht diese im Mössbauer-Spektrum mit der $8 \times 8$-Probe auf und bleibt anteilmäßig relativ konstant bis zur $13 \times 13$-Probe. Das Hyperfein-Feld des Sextetts ist jedoch nicht mit der Pulsanzahl korreliert, dessen Wert liegt zwischen 7,3-8,1 T.

Zwischen der $14 \times 14$ - zur $16 \times 16$-Probe ist im XRD-Spektrum deutlich der Wechsel von amorpher Phase zu einer Vielfalt kristalliner Phasen zu erkennen. Im CEM-Spektrum dagegen vollzieht sich der Wechsel zwischen $13 \times 13$ (annähernd identisch mit der Probe bei $11 \times 12$ Pulsen) und der $14 \times 14$ Pulsen: In den Spektren der $14 \times 14$ - und der $16 \times 16$-Probe treten zusätzlich 2 breite Doubletts hervor, die hauptsächlich (Fe,Cr)-Misch-Carbiden 198 199 zuzuordnen sind und sich schon sehr schwach im XRD-Spektrum der $14 \times 14$-Probe bemerkbar machen. Das XRD-Spektrum konnte jedoch keine eindeutige Aussagen über die Carbid-Zusammensetzung liefern, da es zu viele Reflexe sind, die gemäß PCPDFWIN-Datenbank auch intermetallischen Phasen und Ni-enthaltenden Misch-Carbiden zugeordnet werden können. Die CEMUnterspektren sind in beiden Proben größenordnungsmäßig ungefähr gleicher Intensität. Folglich weisen die Phasen (mind. bis zur CEMS-Tiefe von $150 \mathrm{~nm}$ ) eine ähnliche Zusammensetzung auf. Das breite Sextett repräsentiert sowohl die kristallinen Phasen der $16 \times 16$ - als auch den amorphen Untergrund der 
$8 \times 8$ - und der $14 \times 14$-Puls-Proben. Der Anteil der amorphen Phase ist bei der $14 \times 14$ jedoch höher. So ist zu erklären, dass XRD nur schwache kristalline Reflexe und einen starken amorphen Untergrund zeigt. Im Vergleich zu den Eisenproben können zwei markante Unterschiede festgestellt werden:

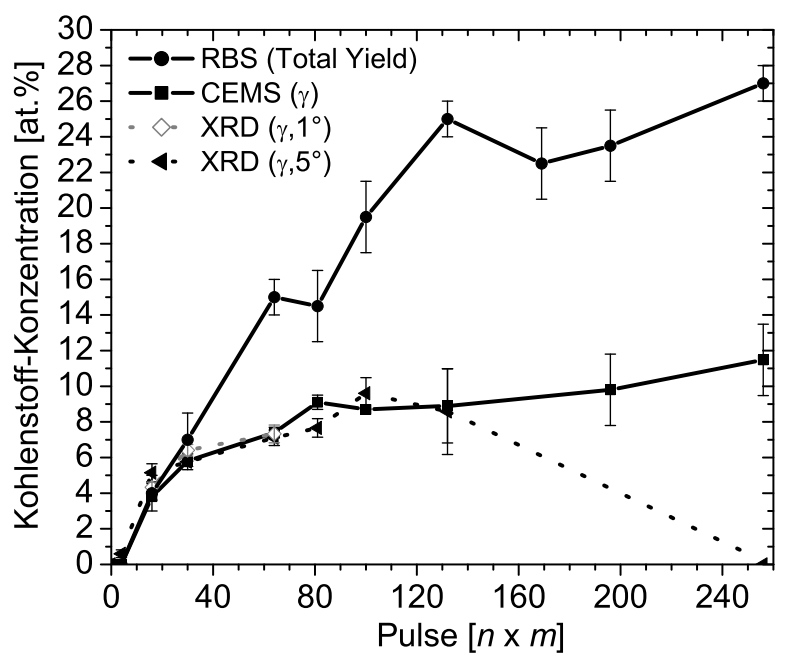

Abbildung 6.6: Der mit verschiedenen Methoden bestimmter Kohlenstoffgehalt im $\gamma$-Fe(C) stimmt relativ gut untereinander überein. Der RBS-Yield beinhaltet den gesamten Kohlenstoffgehalt.

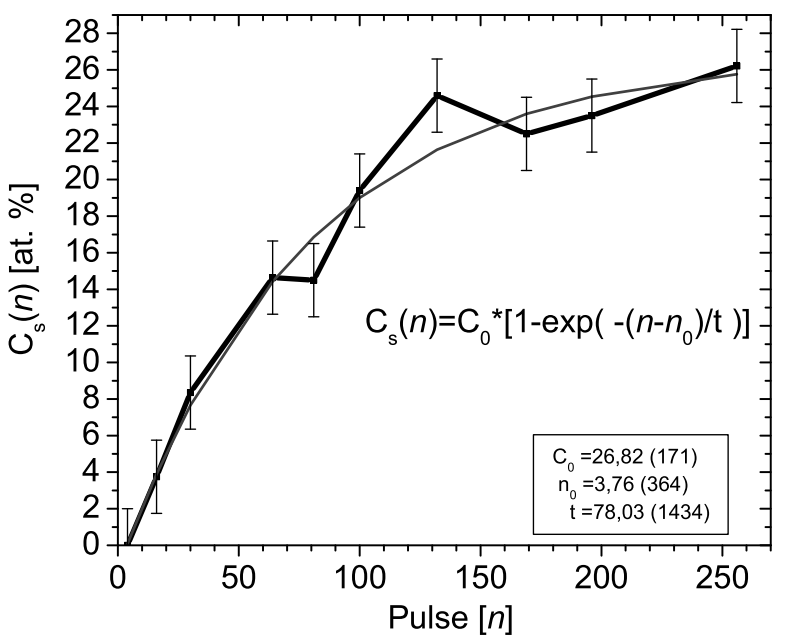

Abbildung 6.7: Der RBS-Kohlenstoffgehalt (Abb. 6.6 wurde durch eine exponentielle Sättigungs-Funktion angepasst und liefert einen maximalen Kohlenstoff-Gehalt von $c_{\max } \approx 26,8$ at.\%.

- Die Phasenentwicklung in der Edelstahlserie ist von der in der Fe-Serien grundlegend verschieden: Es entsteht mit steigendem Kohlenstoff-Eintrag eine amorphe Phase, die sich bei der höchsten Pulszahl von $16 \times 16$ Pulsen in kristalline Phasen umwandelt. Auch wenn bei reinem Eisen unter bestimmten Prozessbedingungen ein erheblicher Anteil von $\gamma-\mathrm{Fe}(\mathrm{C})$ entsteht und dieser als Ausgangs-Phase für die nächsten Pulse dient, ist in keinem Fall eine Amorphisierung festzustellen. Es sind darüber hinaus keine Peaks präsent, die auf die Existenz von $\theta$ - und $\varepsilon$-Carbids schließen lassen.

- Bei Edelstahl kommt es zur Ausbildung des expanded austenite, dessen Gitterkonstanten aufgrund Kohlenstoffaufnahme größer sind als die des Ausgangs-Austenits, das im Edelstahl zudem auch ohne Einlagerung von Kohlenstoff stabil ist. Bei beginnender Kohlenstoffaufnahme bildet sich nicht nur expanded $\gamma-\mathrm{Fe}(\mathrm{C})$, sondern es bleibt auch unreagiertes Grundaustenit übrig. In den Fe-Proben ist kein solcher $\gamma$-Fe(C)-Doppelpeak zu beobachten, da dieser kein Austenit als Ausgangsphase besitzt.

Auffällig ist, dass über die gesamte Reihe hinweg die $\gamma$-Fe-Phase bzw. die (Doppel)Peaks stets vorhanden sind, sowohl in der Tiefe ( $5^{\circ}$-XRD) als auch in den oberflächennahen Schichten (CEMS, $\left.1^{\circ}-\mathrm{XRD}\right)$. Die austenitische Edelstahl-Matrix wird durch den Laserprozess nicht vollständig in die amorphe Phase überführt. Zusätzlich zur Pulsserie bei $\mathrm{p}\left(\mathrm{CH}_{4}\right)=1$ bar ist auch eine Druckserie bei $11 \times 12$ Pulsen untersucht worden. Im Gegensatz zur Fe-Serie, bei der sich verschiedene Phasen bildeten, sind die XRD-Spektren der Proben bei $\mathrm{p}\left(\mathrm{CH}_{4}\right)=0,1 / 0,5 / 1 / 2$ und 4 bar annähernd identisch (Abb. 6.8). Sie zeigen alle den typischen amorphen Untergrund, der auch in der Pulsserie zu erkennen ist.

Ebenso sind die Peaks des Grund- und des expanded austenite vorhanden. Die Kohlenstoff-Profile (Abb.6.9) zeigen für alle Proben einen Kohlenstoff-Gehalt von ca. 24(2) at.\%. Bei der $\mathrm{p}\left(\mathrm{CH}_{4}\right)=2$ bar Probe ist ein erhöhter C-Gehalt auf der Oberfläche zu verzeichnen. Die Oberfläche ist nur bei der $\mathrm{p}\left(\mathrm{CH}_{4}\right)=0,1$ bar Probe schwarz, jedoch nicht in dem Maße wie die entsprechende Fe-Probe. Die CEM-Spektren sind alle identisch mit der $11 \times 12$-Probe der Pulsserie (s. Abb. 6.10). Nur das CEM-Spektrum der 2 barProbe weicht etwas von den Proben der übrigen Drücken ab. Der amorphe Untergrund am wenigsten ausgeprägt. Die besondere Schwierigkeit liegt darin, die breiten Spektren in die Unterspektren aufzulösen und vernünftig anzupassen. Die Spektren bestehen aus einem breiten Sextett, einem Singulett und aus 2 bzw. ab $14 \times 14$ Pulsen aus 4 Doubletts. Die beiden Doubletts und das Singulett sind für die Proben bis $5 \times 6$ Pulse sehr gur anpassbar. Ab $8 \times 8$ Pulsen kommt ein breites Sextett hinzu, was bei gleich gutem Fit 


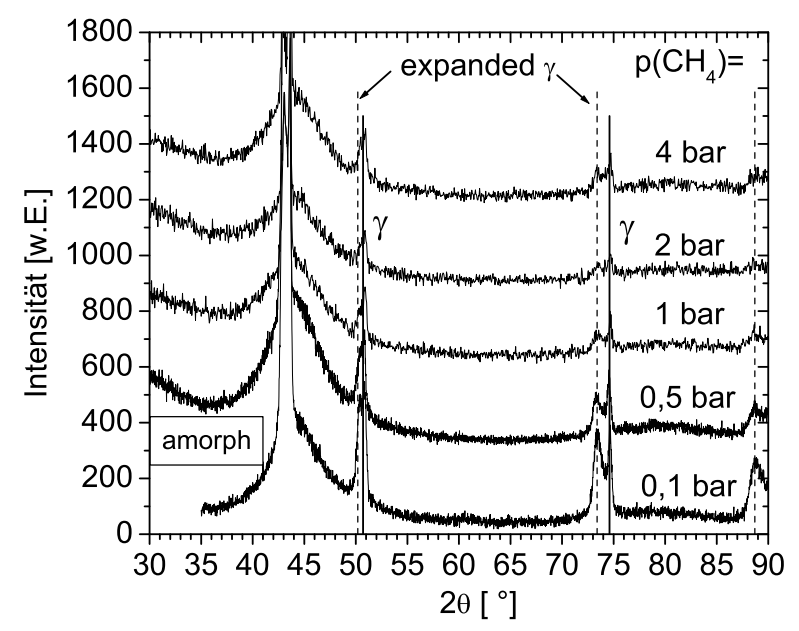

Abbildung 6.8: XRD-Spektren der Edelstahl-Druckserie bei $11 \times 12$ Pulsen. Deutlich zu erkennen ist der amorphe Untergrund und die $\gamma$-Fe(C)-Doppelpeaks (expanded und Grund-Austenit).

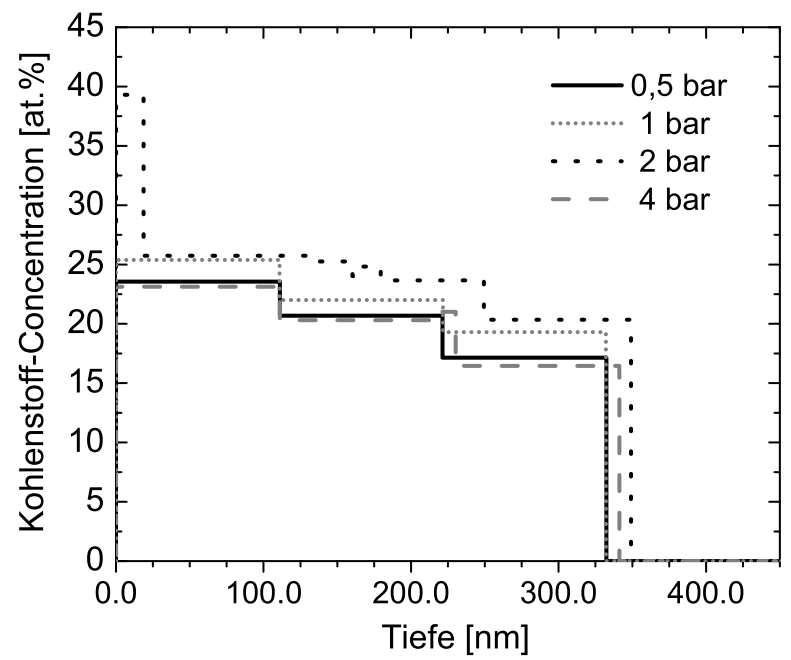

Abbildung 6.9: Kohlenstoffprofil der EdelstahlDruckserie bei $11 \times 12$ Pulsen und $4 \mathrm{~J} / \mathrm{cm}^{2}$.

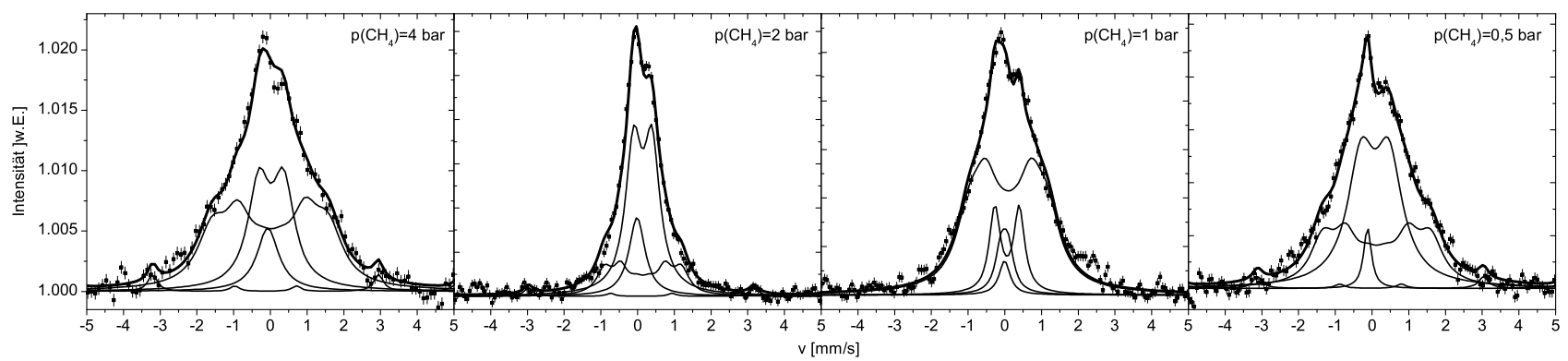

Abbildung 6.10: CEM-Spektren der Edelstahl-Druckserie bei $11 \times 12$ Pulsen und $4 \mathrm{~J} / \mathrm{cm}^{2}$.

die Intensität des schmalen Doubletts und des Singuletts etwas variieren lassen kann. Für die Bestimmung des Kohlenstoffgehalts ist der Fehler jedoch nicht sehr groß, da die Gesamtfläche des Doubletts und des Singuletts nahezu gleich bleibt. Komplizierter wird es bei den Proben ab $14 \times 14$ Pulsen. Hier kommen noch 2 weitere Doubletts hinzu, die (Fe,Cr)-Misch-Carbiden zuzuschreiben sind. Die Parameter der 4 Doubletts und des Singuletts $I S, Q S, \Gamma$ und Intensität können bei vorgegebenem Summenspektrum untereinander umso stärker schwanken, je weniger Fit-Parameter festgehalten wurden. Daher konnten die Hyperfeinparameter nur grob bestimmt werden.

Die magnetischen Eigenschaften der Pulsserie wurde zusätzlich mithilfe des magneto-optischen KerrEffektes (MOKE) bekräftigt (Abb. 6.11). Der Effekt beruht im wesentlichen darauf, dass die Polarisationsebene eines auf die Probe einfallenden Laserstrahles in Abhängigkeit der Magnetisierung $\boldsymbol{m}$ um einen bestimmten Winkel $\delta$ gedreht wird [139 137 138]: $\delta \sim \boldsymbol{m}$. Die Informationstiefe liegt bei Metallen gewöhnlich bei einigen $10 \mathrm{~nm}$ [139]. Ergebnisse mit CEMS können somit nicht unmittelbar verglichen werden. Die Einheit der Abzisse wird in Oersted angegeben $\left(1 \mathrm{Oe}=10^{3} / 4 \pi \mathrm{A} / \mathrm{m}\right)$. Die Ordinate wird in Millivolt des MOKE-Signals gemessen und gibt die relative (Remanenz- und Sättigungs-) Magnetisierung an. Es ist zu erkennen, dass mit steigender Pulszahl die Sättigungsmagnetisierung kontinuierlich ansteigt. Die Magnetisierungskuve von reinem Eisen ist mit der Apparatur nicht aufzunehmen, da die Sättigungsmagnetisierung nicht erfasst werden kann. Die maximale Feldstärke am Ort der Probe liegt bei rund 1400 Oe. Die MOKE-Messungen zeigen im Gegensatz zu den CEM-Spektren (Abb. 6.5) einen Zusammenhang der Pulsanzahl mit der (relativen) Sättigungsmagnetisierung $M_{\text {Sätt }}$ (s. Abb. 6.12), die u.a. ein Maß für die Dichte der ausgerichteten Spins pro Volumeneinheit angibt. Die Entwicklung der Sättigungsmagnetisierung der amorphen Phase kann gut mit einer Exponential-Funktion angepasst werden. Die $16 \times 16$-Probe wurde nicht miteinbezogen, weil dort auch kristalline Phasen zur Gesamtmagnetisierung beitragen. In 


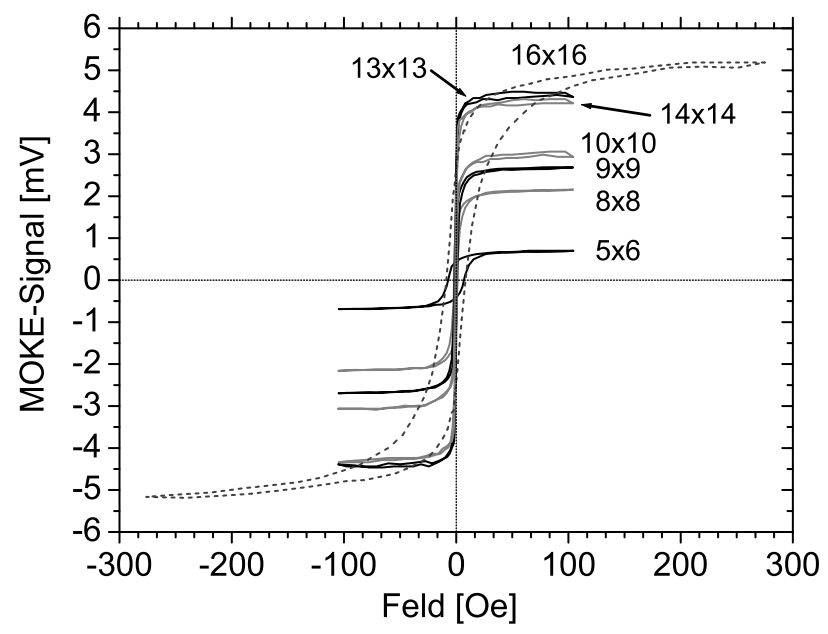

Abbildung 6.11: MOKE-Messungen der Edelstahl-Pulsserie bei 1 bar. Das maximale Feld beträgt 300 Oe.

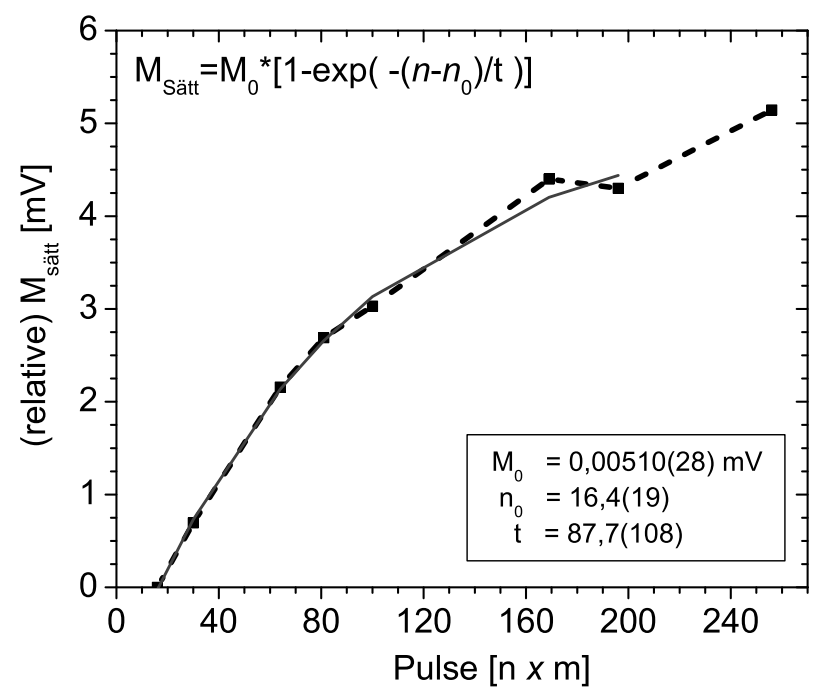

Abbildung 6.12: Die Sättigungsmagnetisierung der Pulsserie in Abhängigkeit von der Pulszahl.

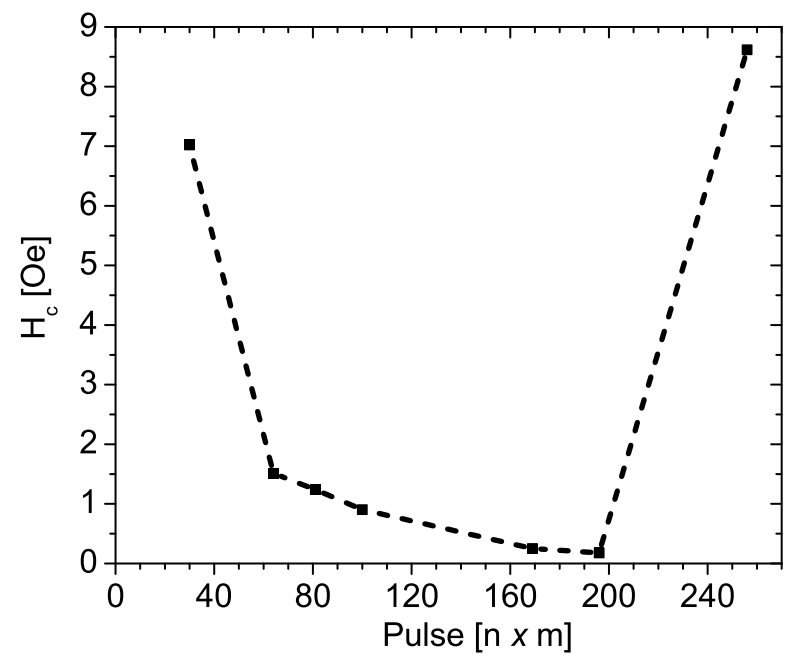

Abbildung 6.13: Das Koerzitivfeld in Abhängigkeit der Pulszahl.

Abb. 6.13 ist das Koerzitivfeld gegen die Pulszahl aufgetragen. Lässt man die 16×16-Probe außen vor, so ergibt sich ein abnehmender Trend des Feldes $H_{c}$ mit der Pulszahl. Sehr viel Faktoren wie chemische, strukturelle, metallurgische und geometrische Beschaffenheit spielen eine Rolle, die das Koerzitivfeld beeinflussen [200], um es beispielsweise mit dem Stress zu korrelieren. Aufgrund fehlender Baufehler wie Versetzungen und Korngrenzen ist generell in amorphen Stoffen das Koerzitivfeld sehr klein und die Hystereskurve sehr schmal, weil dadurch die Bloch-Wände beweglicher sind als in kristallinen Materialien [137], wie in diesem Fall in der 16×16-Probe. Diesen Sachverhalt kann man in Abb. 6.11 gut erkennen. Die $14 \times 14$-Probe zeigt im MOKE amorphes Verhalten, weist jedoch im XRD-Spektrum (s. Abb. 6.2 schon kristalline Reflexe auf. Daraus kann geschlossen werden, dass die kristallinen Phasen unterhalb der amorphen Oberflächenschicht liegen und nicht umgekehrt. Ein weiteres Problem ist, dass aufgrund der geringen Informationstiefe von einigen $10 \mathrm{~nm}$ keine andere Technik zum Vergleich herangezogen und deren Ergebnisse (z.B. der Stress) damit verknüpft werden können.

\subsection{EXAFS}

Die Spektren der EXAFS-Messungen sind in Abb. 6.14 dargestellt. Die reduzierten Peaks der höheren Koordinationsschalen zeigen aufgrund fehlender Fernordnung die Amorphizität bzw. Unordnung auf. Um die atomare Nahordnung einzelner Atome im Edelstahl zu bestimmen und den amorphen Charakter 


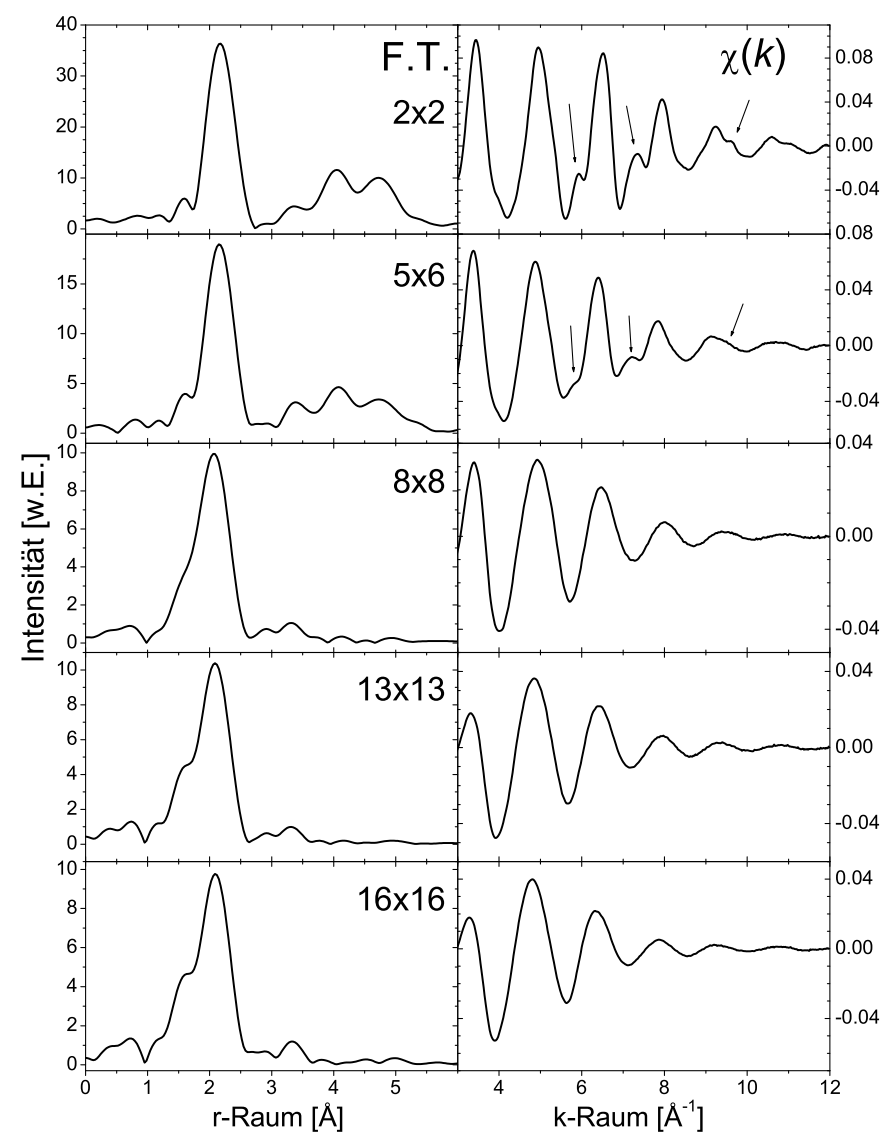

Abbildung 6.14: Die EXAFS-Oszillationen $\chi(k)$ (rechts) und die Fourier-Transformierten $\mathcal{F}\left\{\chi(k) \cdot k^{3}\right\}$ (links). Die Satelliten in der EXAFS der $2 \times 2$ - und $5 \times 6$-Probe (Pfeile) werden durch die relativ starken Beiträge der zweiten und höheren Schalen verursacht. Man beachte die Intensität der Fourier-Transformierten.

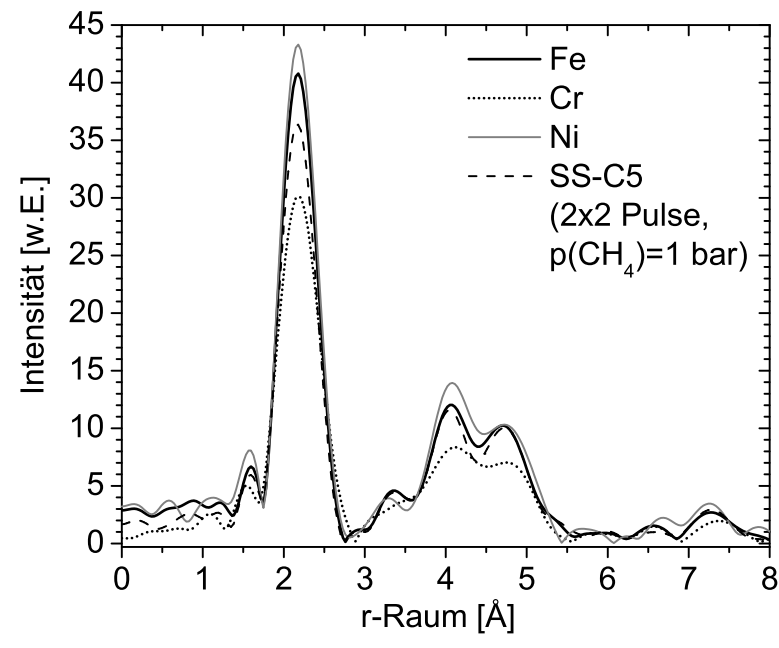

Abbildung 6.15: Fourier-Transformierte der $\chi(k)$ der Kanten von $\mathrm{Fe}, \mathrm{Cr}$ und $\mathrm{Ni}$ im unpoliertem / unbehandeltem Edelstahl bzw. von Fe in der ReferenzProbe.

zu stützen, wurden die Synchrotron-Messungen am L.U.R.E. (Beamline DCI-42) in Orsay/Paris bzw. am E.S.R.F. in Grenoble durchgeführt. Dazu wurden überwiegend die Photoelektronen der Fe $K$-Kante $\left(E_{0}=7112,0 \mathrm{eV}[201 \mid)\right.$ bei Raumtemperatur im total electron yield-Modus (TEY) in einem Energie-Bereich von 7000-7800 eV in $1 \mathrm{eV}$ Schritten aufgenommen. Die Auswertung erfolgt mit der VIPER-Software [119]. Die Informations-Tiefe $d$ für die Elemente ergibt sich nach 202 folgendermaßen: Fe: $84 \mathrm{~nm}, \mathrm{Cr}$ : $70 \mathrm{~nm}$ und Ni: $93 \mathrm{~nm}$. Für Edelstahl wird demnach der Wert von $70 \mathrm{~nm}(\mathrm{Cr})$ angenommen. Der atomare Absorptionskoeffizient $\mu_{0}$ wird mit einem kubischen Spline simuliert. Die gemessenen EXAFS-Oszillationen $\chi(k)$ werden mit $k^{3}$ gewichtet und im Bereich $3<k<12 \AA^{-1}$ Fourier-Transformation durchgeführt (s. Abb. 6.14). Abschneide-Effekte in der Fourier-Transformation, die durch das begrenzte $k$-Intervall entstehen, werden durch ein Hanning-Fenster minimiert [112 117]. Die Oszillationen im Bereich $0<r<1,7 \AA$ rühren von diesen Effekten her. Die erste Koordinationsschale wird im Bereich 1, $18<r<2,7 \AA$ rücktransformiert, um nur den EXAFS-Beitrag der nächsten Nachbarn zu erhalten. Die zweite und höheren Koordinationsschalen verursachen die Satelliten (angedeutet durch die Pfeile) in der EXAFS der $2 \times 2$ und noch zum Teil in der $5 \times 6$-Probe. Bei der Fourier-Rücktransformation werden die höheren Schalen durch das eingeschränkte $r_{\min }-r_{\max }$-Intervall ausgefiltert, wodurch die Satelliten entfallen.

Als Referenz wurde die Probe $2 \times 2$ Pulsen benutzt. In dieser Probe ist zum einen noch ein nicht nachzuweisender Anteil Kohlenstoff inkorporiert; die XRD- und RBS Spektren im Vergleich zum unbehandelten und unpolierten Edelstahl sind identisch. Zum anderen wird die Qualität der Oberfläche erhöht, weil durch die geringe Anzahl Pulse von der Oberfläche spannungs-induzierter Martensit, der durch das Polieren entsteht, und Oxide entfernt werden.

In Abb. 6.15 sind die Fourier-Transformierten der Elemente $\mathrm{Fe}, \mathrm{Cr}$ und $\mathrm{Ni}$ im Vergleich zu Fe und der Referenz gezeigt. Dabei sind die Energien für die Cr $K$-Kante $E_{0}=5989,2 \mathrm{eV}$ und für die Ni $K$-Kante 
$E_{0}=8332,8 \mathrm{eV}$ [201]. Die unterschiedlichen Intensitäten $I$ rühren von den unterschiedlichen Rückstreuamplituden der einzelnen Elemente her [203]. Die atomare Umgebung der einzelnen Elemente weisen dasselbe strukturelle Muster auf. Die Fe-Kanten des Edelstahls und der benutzten Referenz sind sehr ähnlich, weichen jedoch im Peak der 1. Schale etwas stärker ab. Leichte strukturelle Unterschiede in der Oberfläche wie z.B. Atomumordnung durch Oxidbildung kommen dafür in Frage. Die EXAFS-Oszillationen $\chi(k)$ der

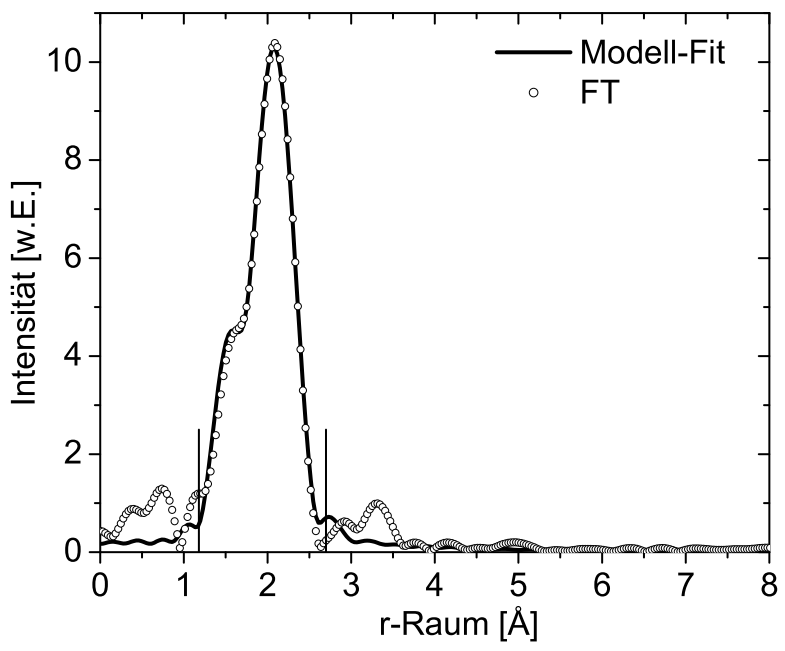

Abbildung 6.16: Fourier-Transformierte ( $\circ \mathrm{FT})$ des $\chi(k)$

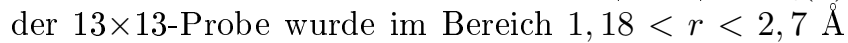
rücktransformiert (o BFT in Abb. 6.17). Der Fit der drei gaussförmigen RDFs (Abb. 6.18) ist als Linie (-) dargestellt.

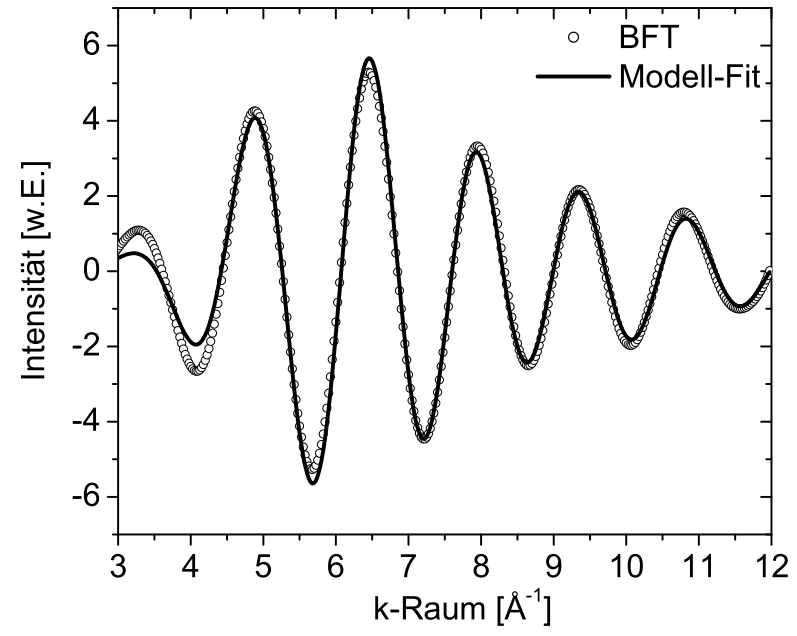

Abbildung 6.17: Die Rücktransformierte (o) ist mit drei gaussförmigen RDFs angefittet (-), die eine Fe-CSchale und zwei Fe-M-Schalen repräsentieren.

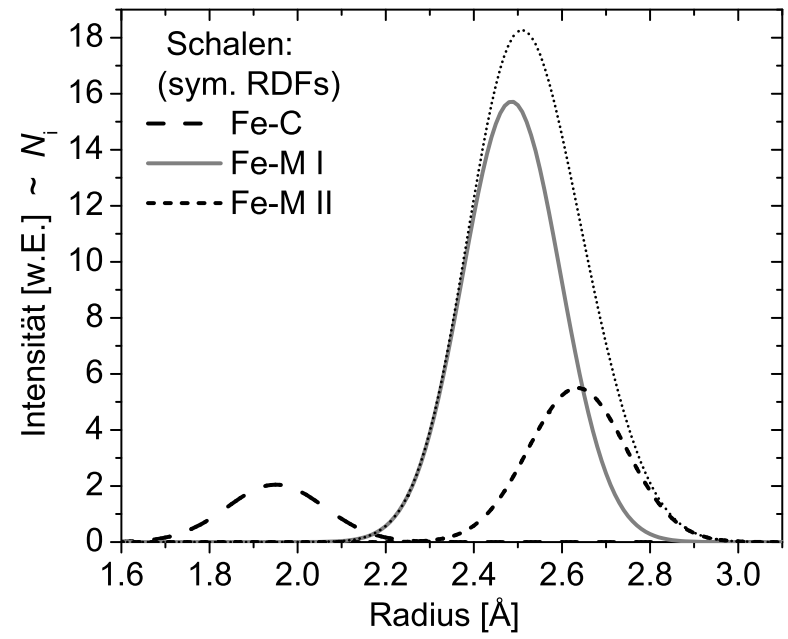

Abbildung 6.18: Die Intensität der gaussförmigen RDFs ist der Anzahl der nächsten Nachbarn $N_{i}$ proportional. Die beiden Fe-M-Schalen bilden die asymmetrische RDF $g_{\text {asym }}(\cdots)$.

Referenz-Fe-Kante werden zunächst mit dem Programm Feff 8.1 simuliert 204 205]. Dieses Programm errechnet die Phase $\phi(k)$ und effektive Streuamplitude $F(k)$, die der Messung nicht zugänglich sind. Als notwendige Eingabe-Datei für FEFF wird eine Liste der Atom-Koordinaten benötigt, die das Programm Aтомs generiert [206]. In der Simulation werden der Parameter $\sigma^{2}$, Gitterkonstante $a, E_{i}$ und $E_{r}$ an das gemessene Spektrum angepasst. Der reale Energieanteil $E_{r}$ verschiebt den Energie-Nullpunkt nach $k \rightarrow \sqrt{k^{2}-E_{r} \cdot 2 m_{e} / \hbar^{2}}$ (s. Gl. (3.58), hier ist $E_{r}=E_{\text {bind }}$ ), der imaginäre Energieanteil $E_{i}$ beschreibt die instrumentelle Linienverbreiterung. Der Unordnungs-Parameter wurde zu $\sigma^{2}=0,0046 \AA^{2}$, die EnergieVerschiebung zu $E_{r}=0,4 \mathrm{eV}$ bestimmt. In Abb. 6.14 ist ersichtlich, dass mit steigender Pulszahl bzw. Amorphizität die EXAFS-Amplitude abnimmt, was durch eine starke Zunahme des Parameters $\sigma^{2}$ aufgrund struktureller Unordnung verursacht wird. 
Mit EXAFS ist auch möglich, in ungeordneten bzw. amorphen Materialien die nächsten Nachbarn und die (mittleren) Abstände vom Zentralatom zu berechnen. Nur die nächsten Nachbarn und damit der erste Peak spielen bei amorphen Substanzen eine Rolle. Der übliche Weg dabei ist die Einführung asymmetrischer radialer Verteilungs-Funktionen (RDF) [117, 112]. Die Gl. 3.60) schreibt sich dann in der Form:

$$
\chi(k)=\frac{1}{k} \sum_{i} S_{0}^{2}(k) F_{i}(k) \int \underbrace{P\left(r_{i}\right) \frac{e^{-2 r_{i} / \lambda}}{r_{i}^{2}}}_{\text {asym. RDF }} \cdot \sin \left(2 k r_{i}+\phi_{i}(k)\right) d r_{i},
$$

$P\left(r_{i}\right)$ ist die Wahrscheinlichkeit, das $i$-te Atom im Bereich zwischen $r_{i}$ und $r_{i}+d r_{i}$ zu finden. Zur Auswertung mit VIPER wird folgende Formel mit der allgemeinen RDF $g\left(r_{i}\right)$ angewandt [119]:

$$
\chi(k)=\frac{1}{k} \sum_{i} S_{0}^{2}(k) F(k) \int_{r_{\min }}^{r_{\max }} \frac{g_{i}(r)}{r^{2}} \sin (2 k r+\phi(k)) d r \quad \operatorname{mit}: \int_{r_{\min }}^{r_{\max }} g_{i}(r) d r=N_{i}
$$

Eine asymmetrische RDF kann u.a. durch Superposition mehrerer symmetrischer Gauss-RDFs erzeugt werden [117.

Nun besteht das Problem darin, dass keine amorphe Referenz bei der Messung vorhanden war. Die Phase $\phi(k)$ und die Amplitude $F(k)$ sind nur für die kristalline $2 \times 2$-Referenz-Probe mit FEFF berechnet worden. Man kann jedoch unter gewissen Umständen $\phi(k)$ und $F(k)$ von bekannten Modellen bzw. Referenzen übernehmen und auf die unbekannte Probe übertragen. Diese sog. phase/amplitude transferability ist dann gültig, wenn überwiegend Einfach-Streu-Prozesse auftreten. Da hier nur die relevanten nächsten Nachbarn betrachtet werden, ist diese Bedingung erfüllt [117]. Die Abb. 6.16 6.18 zeigen die Resultate der Anpassungs- und Transformations-Schritte im Detail. Die Energieverschiebung $E_{r}$ wurde aus der FEFFSimulation der $2 \times 2$-Probe übernommen. Zunächst wurde -wie bereits oben erwähnt- das gemessene $\chi(k)$ der $13 \times 13$-Probe mit $k^{3}$ gewichtet und im Intervall $0<r<8 \AA$ Fourier-transformiert ( $\circ$ BT in Abb. 6.16). Der erste, stark ausgeprägte Peak wurde im Bereich 1,18<r<2,7 Fourier-rücktransformiert (o BFT in Abb. 6.17): Die Oszillationen im $k$-Raum wurden durch drei Gauss-RDFs angepasst, deren Summe (-) der Modell-Fit ist. Im $r$-Raum (Abb. 6.16) ist nochmal der entsprechende Model-Fit (-) im Vergleich zur ursprünglichen Fourier-Transformierten ( $\mathrm{B}$ B) dargestellt.

Den RDFs können drei Schalen zugewiesen werden: In der ersten Koordinationsschale befinden sich im Abstand von ca. 1,95 A C-Atome als nächsten Nachbarn, die Fe-C-Schale. Die beiden Schalen mit den Metallatomen (Fe,Cr,Ni,Mo) als nächste Nachbarn befinden sich jeweils im Abstand von ca. 2,49 A (Fe-M I) bzw. 2,63 $\AA$ (Fe-M II). Die Summe der beiden Fe-M-RDFs führt zu einer asymmetrischen RDF $g_{\text {asym }}$ (Abb. 6.18). Die Unordnungs-Parameter $\sigma^{2}$ der RDFs wurden untereinander gleichgesetzt und gemeinsam angepasst. Die Intensitäten der RDFs sind zu den nächsten Nachbarn $N$ proportional.

Im Bereich bis $k \approx 4,3 \AA^{-1}$ gab es Anpassungsschwierigkeiten (s. Abb. 6.17), deren Ursache nicht klar ist. Diese Fehlanpassung ist z.B. nur mit sehr vielen nächsten Nachbarn $(N>20)$ bei ähnlichen Abständen korrigierbar, was jedoch unphysikalisch ist. Dadurch wurden je nach Fit verschiedene Parameter erhalten, von denen die nächsten Nachbarn der ersten Fe-M-Schale am stärksten schwankten. Von jeweils vier Fits sind die Mittelwerte in Tab. 6.2 aufgelistet. Wegen der bleibenden Fehlanpassung in den Fits ist

Tabelle 6.2: Angepasste und gemittelte Parameter der $13 \times 13-$ Probe: $\bar{N}:$ Nächste Nachbarn, $\bar{x}$ : Distanz der Schale zum Zentralatom $[\AA]$ und $\overline{\sigma^{2}}$ : Unordnungs-Parameter $\left[\AA^{2}\right]$.

\begin{tabular}{|c||c|c|c|}
\hline Schale & Fe-C & Fe-M I & Fe-M II \\
\hline \hline $\bar{x}[\AA]$ & $1,953(5)$ & $2,488(8)$ & $2,625(34)$ \\
\hline $\bar{N}$ & $1,46(5)$ & $10,6(10)$ & $3,67(3)$ \\
\hline$\overline{\sigma^{2}}\left[\AA^{2}\right]$ & $0,01237(36)$ & $0,01237(36)$ & $0,01237(36)$ \\
\hline
\end{tabular}

es nicht möglich, hundertprozentig auf die ermittelten Werte zu vertrauen. Der generelle Aspekt dieser Prozedur bleibt jedoch erhalten. Der Abstand der Fe-C-Schale wurde zu 1,95 $\AA$ errechnet, was eine sehr gute Übereinstimmung mit dem kovalenten Abstand (Summe der kovalenten Radien) von 1,94 $\AA$ ist. Die Eigenschaften der Schalen Fe-M I/II sind: 
a) 14 nächste Nachbarn: Neben Fe und $\mathrm{C}$ sind noch Ni, Cr und Mo in gewissen Atomzahl-Verhältnissen präsent, die zudem noch unterschiedliche Streu-Amplituden besitzen. Es ist nicht auszuschließen, dass sich auch mehrere C-Atome in den Fe-M-Schalen befinden. Wieviel und welche Atome nun tatsächlich vorhanden sind, lässt sich nicht angeben.

b) Distanzbereich von ca. 2,49-2,63 A: Aufgrund der unterschiedlichen Atomgrößen und Abständen zum Zentralatom wird diese asymmetrische Verteilung (RDF) verursacht. Der gewichtete, mittlere Abstand der zusammengesetzten Schale beträgt $\bar{r}_{\text {gew }}=\int g_{\text {asym }} r d r / g_{\text {asym }} d r=2,525 \AA$.

$\mathrm{Zu}$ der asymmetrischen Verteilung ist noch anzumerken, dass diese ebenso gut mit mehr als 2 GaussRDF mit entsprechenden Radien und Intensitäten bzw. Anzahl der nächsten Nachbarn konstruiert werden kann.

Was in den Spektren in Abb. 6.14 auffällt, ist die Tatsache, dass die EXAFS der amorphen $13 \times 13-$ und $14 \times 14$-Probe (wegen Gleichheit nicht dargestellt) mit der kristallinen $16 \times 16$-Probe fast übereinstimmt. XRD-Strukturell (s. Abb. 6.2) und auch von den magnetischen Eigenschaften (s. Abb. 6.11) her sind die Proben zweifellos verschieden. In der 16×16-Probe sind Kristallite von Phasen unterschiedlichster Struktur enthalten. Die Fernordnung eines bestimmten Kristallsystems über mehrere Koordinationsschalen hinweg wird durch die eines anderen Kristallsystems zerstört. In der Fernordnung scheinen die umgebenen Atome unregelmäßig und zufällig angeordnet, wie es bei ungeordneten bzw. amorphen Substanzen der Fall ist.

\subsection{Mikrohärtemessung der Pulsserie}

Die Härte der Edelstahlproben der Pulsserie ist in Abb. 6.19 dargestellt: Aufgrund der Bildung von stress-

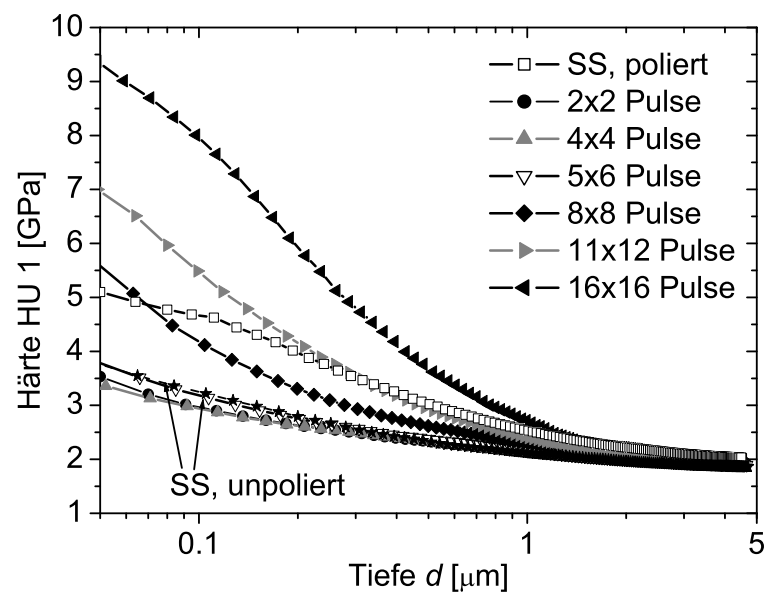

Abbildung 6.19: Die Universalhärte der Edelstahlproben der Pulsserie. Die Härte des polierten, unbestrahlten Edelstahls liegt zwischen Proben niedriger $(\leq 8 \times 8)$ und hoher $(\geq 11 \times 12)$ Pulszahl.

induziertem $\alpha^{\prime}$-Fe ist der polierte Edelstahl härter als der unpolierte. Die Proben, die mit $\leq 8 \times 8$ Pulsen bestrahlt wurden, sind weicher als der polierte Edelstahl und sind mit dem unpolierten Edelstahl zu vergleichen, dessen Härte mit der 5×6-Probe annähernd identisch ist. Durch die Bestrahlung verschwindet einerseits das $\alpha^{\prime}$-Fe durch Umschmelzen/Rekristallisation, andererseit entsteht $\gamma$ - $\mathrm{Fe}(\mathrm{C})$, das die Härte herabsetzt. Die $8 \times 8$-Probe ist wegen der Bildung der beginnenden amorphen Phase (Abb. 6.5 und 6.19) etwas härter, liegt dennoch unter dem polierten Edelstahl. Die $11 \times 12$-Probe besitzt den höchsten amorphen Anteil und hat dementsprechend die höchste Härte der amorphen Proben. Die höchste Härte der Serie zeigt die kristalline $16 \times 16$-Probe, die durch verschiedenen Carbide verursacht werden. In Tab. 6.3 sind die Härtewerte für die Proben ab $8 \times 8$ Pulsen zusammengefasst, die sich analog den Fe-Proben nach Gl. 5.6 errechnen. Für kleinere Pulszahlen ist das Modell der Härtenbestimmung (s. Kap. 5.14) nicht anwendbar, da entweder $d / t<0,1$ ist oder $d / t>0,1$ ist zwar erfüllt, aber in Tiefen $t$, die beträchtlich größer sind als die zugrundegelegte Aufschmelztiefe von ca. $1 \mu \mathrm{m}$. Ein noch akzeptables Beispiel dieses Sachverhalts zeigt die Härte des polierten Edelstahls in Tab. 6.3. Hier ist $d / t<0,1$, doch $1,11 \mu \mathrm{m}$ ist der kleinste Wert für die Aufschmelztiefe, für $d / t>0,1$ ist $t$ jedoch zu groß. Der Härte des Edelstahls beträgt im Mittel 1840(86) MPa (im Vergleich zu Eisen mit 1260(24) MPa). 
Tabelle 6.3: Härtewerte der Edelstahl-Pulsserie. Es sind $H_{s}$ und $H_{c}$ die Härten des Substrates bzw. der OberflächenSchicht der Dicke $t, k$ Deformationsparameter.

\begin{tabular}{|c||c|c|c|c|}
\hline Pulse & $k$ & $H_{s}[\mathrm{MPa}]$ & $H_{c}[\mathrm{MPa}]$ & $t[\mu \mathrm{m}]$ \\
\hline \hline $8 \times 8$ & 0,1 & $1890(3)$ & $3804(31)$ & $1,05(3)$ \\
\hline $\begin{array}{c}\text { Edelstahl, } \\
\text { poliert }\end{array}$ & 0,1 & $1928(5)$ & $4605(19)$ & $1,11(1)(d / t \simeq 0,086)$ \\
\hline $11 \times 12$ & 0,1 & $1900(3)$ & $4222(18)$ & $1,41(2)(d / t \simeq 1,06)$ \\
\hline $16 \times 16$ & 0,1 & $1705(6)$ & $4967(23)$ & $1,02(1))$ \\
\hline
\end{tabular}

\subsection{Bestrahlung in gemischten Gasen, Stickstoff und Ammoniak}

Die Bestrahlung der Proben in einem Gemisch aus Gasen findet bei $3,7 \mathrm{~J} / \mathrm{cm}^{2}$ und $11 \times 12$ Pulsen statt. Besteht die reaktive Atmosphäre aus Gasen mit $\mathrm{p}\left(\mathrm{N}_{2}\right)=0,5$ bar $/ \mathrm{p}\left(\mathrm{CH}_{4}\right)=0,5$ bar, bildet sich zwar auch ein amorpher Untergrund, wie es gewöhlich bei reinem Methan der Fall ist. Dieser ist jedoch nicht so ausgeprägt wie bei reinem Methan. Benutzt man gar ein Gemisch mit $\mathrm{p}\left(\mathrm{N}_{2}\right)=0,5$ bar $/ \mathrm{p}\left(\mathrm{NH}_{3}\right)=0,5$ bar, so ist der Untergrund nicht mehr wahrzunehmen (Abb. 6.20). Die XRD-Spektren der Proben, die ohne

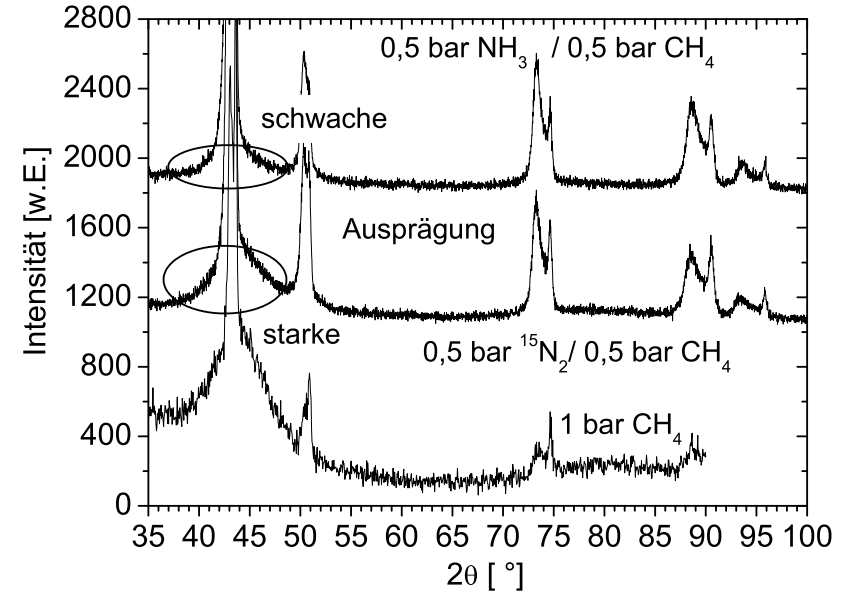

Abbildung 6.20: XRD-Spektren ( $5^{\circ}$ g.i.) der Proben, die in einem Gasgemisch aus je 0,5 bar $\mathrm{CH}_{4}$ und 0,5 bar $\mathrm{N}_{2}$ bzw. $\mathrm{NH}_{3}$ bei $11 \times 12$ Pulsen bestrahlt wurde.

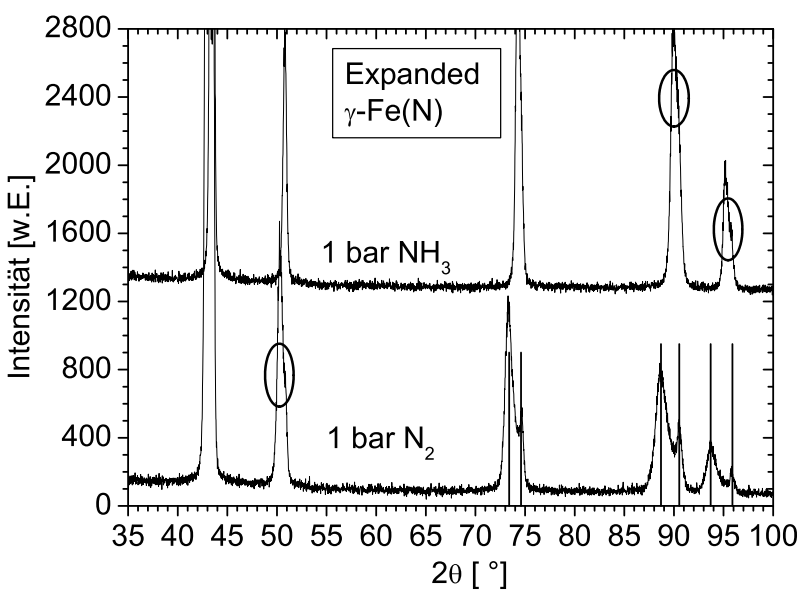

Abbildung 6.21: XRD-Spektren ( $5^{\circ}$ g.i.) der Proben, die bei jeweils 1 bar $\mathrm{N}_{2}$ oder $\mathrm{NH}_{3}$ und $11 \times 12$ bestrahlt wurden. Man erkennt deutlich die Bildung des expanded austenite beim $\mathrm{N}_{2}$. Die Ovale deuten den sehr schwach ausgeprägten Doppelpeak an.

Methan-Zusatz unter gleichen Bedingungen mit je 1 bar $\mathrm{N}_{2}$ und $\mathrm{NH}_{3}$ bestrahlt wurden, zeigen einen markanten Unterschied (s. Abb. 6.21) zu den $\mathrm{CH}_{4}$-Proben: Inkorporierter Stickstoff verursacht keine Bildung einer amorphen, magnetischen Phase. Die Doppel-Peaks der $\mathrm{N}_{2}$-Probe lassen auf einen N-Gehalt von 8,47(49) at.\% schließen; wie der Kohlenstoff verursacht der Stickstoff durch Einlagerung eine Gitteraufweitung. Die Gl.( 6.1) ist analog auch auf den Stickstoffgehalt anwendbar [144]. Der Peak-Abstand der $\mathrm{NH}_{3}$-Probe ist demgegenüber sehr gering und nur im hohen Winkelbereich schwach zu erkennen. Der $\mathrm{N}$-Gehalt beträgt hier nur 2,89(58) at.\%. Die Bindungsenergie des $\mathrm{NH}_{3}$ beträgt $3 \cdot 389 \mathrm{~kJ} / \mathrm{mol}$ und ist höher als die der N-N-Bindung mit $941 \mathrm{~kJ} / \mathrm{mol}$ [45], was letztlich zu einer geringeren Stickstoff-Aufnahme führt. Wie auch $\mathrm{CH}_{4}$ ist $\mathrm{NH}_{3}$ molekular nicht in (flüssigem) Eisen löslich. Damit Stickstoff inkorporiert werden kann, muss sich die Stickstoffverbindung in Stickstoff zerfallen. Das Löslichkeitsverhalten von $\mathrm{N}_{2}$ ist bereits ausführlich untersucht worden [44]. Inwiefern der Wasserstoff, der gemäß $2 \mathrm{NH}_{3} \rightarrow 2 \mathrm{~N}_{2}+3 \mathrm{H}_{2}$ entsteht, bei der Phasenbildung eine Rolle spielt und in welchem Maße den enormen Unterschied in der NKonzentration verursacht, ist unbekannt. Vergleicht man die XRD- und CEM-Spektren der $\mathrm{N}_{2} / \mathrm{CH}_{4}$ - und $\mathrm{NH}_{3} / \mathrm{CH}_{4}$ (Abb. 6.22 und 6.20), so fällt auf, dass nur die Probe einen merklichen amorphen Untergrund zeigt, die das leichter lösliche $\mathrm{N}_{2}$ als Komponente enthält. Andererseits sind die Doppel-Peaks der beiden Gasmischungen $\mathrm{N}_{2} / \mathrm{CH}_{4}$ bzw. $\mathrm{NH}_{3} / \mathrm{CH}_{4}$ nahezu mit der $\mathrm{N}_{2}$-Probe identisch und ergibt einen maximalen 


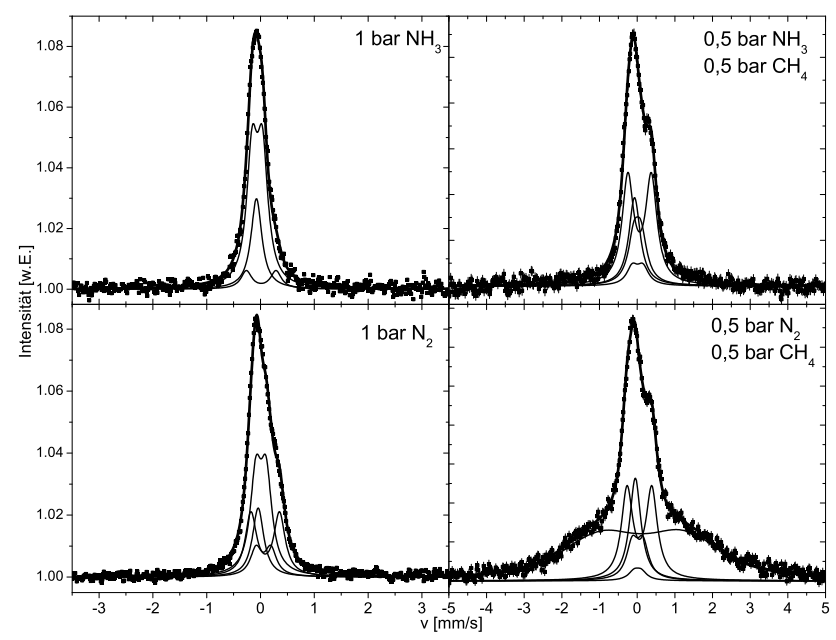

Abbildung 6.22: CEM-Spektren der Proben, die bei 1 bar $\mathrm{N}_{2}, \mathrm{NH}_{3}$ und Gemischen aus je 0,5 bar $\mathrm{N}_{2} / \mathrm{CH}_{4}$ und $\mathrm{NH}_{3} / \mathrm{CH}_{4}$ bestrahlt wurden. Nur die $\mathrm{N}_{2} / \mathrm{CH}_{4}$-Probe zeigt einen amorphen Untergrund. Anzahl der Pulse: $11 \times 12$, $\mathrm{H}=3,7 \mathrm{~J} / \mathrm{cm}^{2}$.

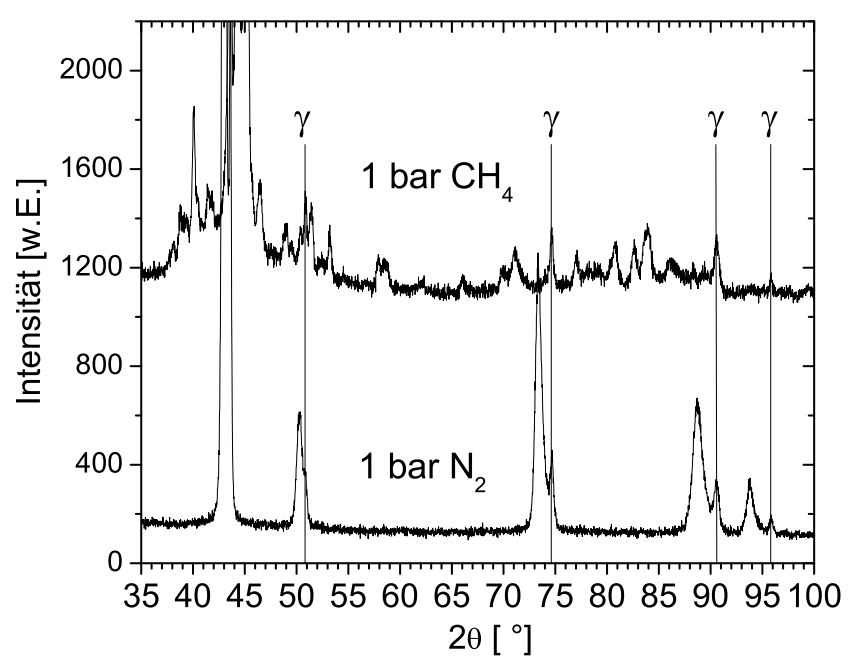

Abbildung 6.23: XRD-Spektren ( $5^{\circ}$ g.i.) der Proben, die unter $\mathrm{p}\left(\mathrm{CH}_{4}\right)=1$ bar und $\mathrm{p}\left(\mathrm{N}_{2}\right)=1$ bar mit $16 \times 16$ Pulsen bestrahlt wurden. Die Linien geben die Positionen des Grundaustenits an.

C/N-Gehalt von ca. 8,5 at.\%. Das $\mathrm{N}_{2}$ zeigt eine höhere Tendenz expanded austenite zu bilden und sich somit eine Lösung einzugehen als $\mathrm{NH}_{3}$. Hat das expanded austenite der $\mathrm{N}_{2} / \mathrm{CH}_{4}$-Probe den maximalen, interstitiellen Anteil an $\mathrm{C} / \mathrm{N}$ von ca. 8,5 at.\% aufgenommen und ist dadurch zunächst gesättigt, so bildet der zusätzlich inkorporierte Kohlenstoff die amorphe Phase. Die $\mathrm{NH}_{3} / \mathrm{CH}_{4}$-Probe hingegen scheint, die Bildung der amorphen Phase und somit weitere Kohlenstoffaufnahme zu hemmen, was an der schwach ausgeprägten amorphen Phase sowohl im XRD- als auch im CEM-Spektrum erkennbar ist. Eine wichtige Bemerkung zum Ammoniak sollte hier gemacht werden:

Die Abb. 6.23 zeigt, dass die $\mathrm{N}_{2}$-Probe, die mit $16 \times 16$ Pulsen bestrahlt wurde, im Wesentlichen der $11 \times 12$-Probe entspricht und im Gegensatz zur $\mathrm{CH}_{4}$-Probe keinerlei kristallinen Phasen (Nitride) aufweist: Ein bedeutender Punkt hierbei ist, dass $\mathrm{N}$ im Gegensatz zu $\mathrm{C}$ ausgasen kann. In der $\mathrm{CH}_{4}$-Probe kommt es bei einer bestimmten Konzentration zur Ausbildung von Fe-, Cr- oder anderen Mischcarbiden, weil mehr Kohlenstoff in den Stahl inkorporiert wird als eigentlich unter Normalbedingungen möglich ist (max. 8,3 at.\%). Diese Konzentration liegt gemäß RBS-Analyse (s. Abb. 6.6 bei ca. 25 at.\%. 


\subsection{Anlassen der Edelstahl-Probe}

Die Probe, die bei $\mathrm{p}\left(\mathrm{CH}_{4}\right)=1$ bar und $11 \times 12$ Pulsen bestrahlt worden war und einen breiten amorphen Untergrund zeigt, wurde in einem Vakuum-Ofen für jeweils 1 Stunde bei $100^{\circ} \mathrm{C}, 200^{\circ} \mathrm{C}, 350^{\circ} \mathrm{C}$ und $500^{\circ} \mathrm{C}$ angelassen. Ziel war es, zu bestimmen, ob sich durch die thermisch aktivierten Rekristallisationsprozesse die ursprüngliche fcc-Struktur wieder ausbildet und welche Carbide dabei entstehen. Abb. 6.24 zeigt

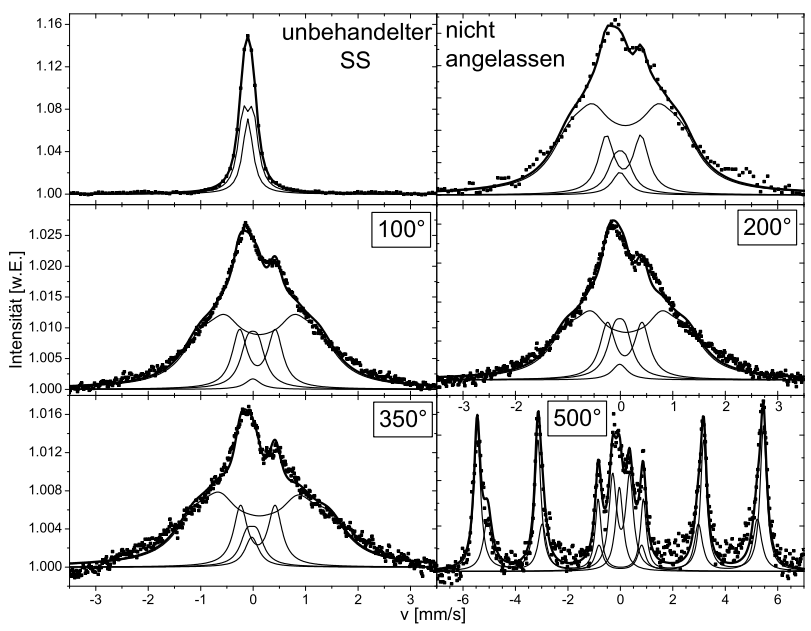

Abbildung 6.24: CEM-Spektren der angelassenen Edelstahls, das bei $\mathrm{p}\left(\mathrm{CH}_{4}\right)=1$ bar und $11 \times 12$ Pulsen bestrahlt wurde. Bei $500^{\circ} \mathrm{C}$ tritt die fcc-Struktur wieder auf.

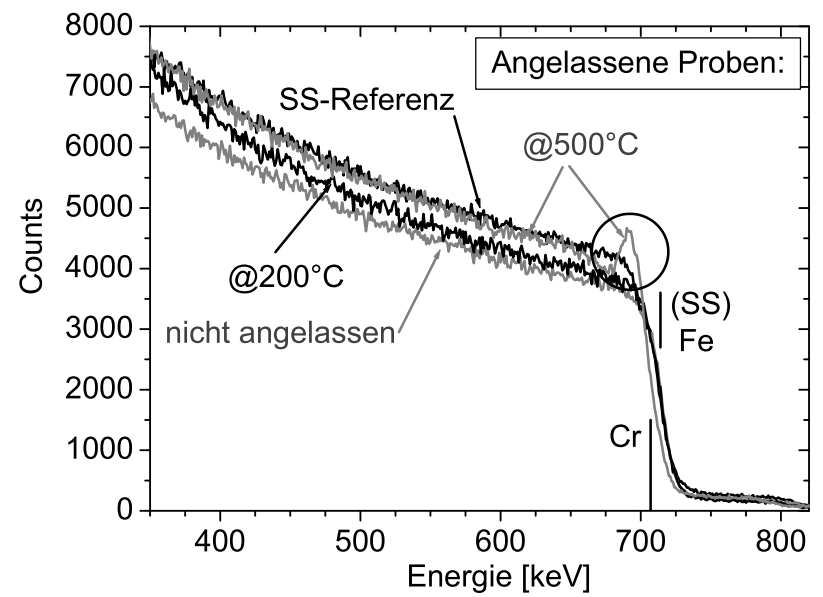

Abbildung 6.25: RBS-Spektrum der Referenz-Probe und der bei $500^{\circ} \mathrm{C}$ angelassenen Probe $\left(\mathrm{p}\left(\mathrm{CH}_{4}\right)=1\right.$ bar und $11 \times 12$ Pulsen).

die CEM-Spektren der Serie. Von $100^{\circ} \mathrm{C}$ bis $350^{\circ} \mathrm{C}$ sind die Spektren nahezu identisch und bestehen überwiegend aus dem breiten magnetischen Unterspektrum. Bei $500^{\circ} \mathrm{C}$ tritt neben den geringen RBSKohlenstoffgehalt von ca. 4,8(10) at.\% eine grundlegend andere Struktur auf: Es sind 2 schmale Sextetts und das Singulett-Doublett-Paar des $\gamma$-Fe(C) vorhanden. Doubletts, die den (Fe,Cr)-Carbiden zugeordnet werden könnten, sind nicht auflösbar bzw. in ihrer Intensität zu klein. Die Hyperfeinfelder unterscheiden sich vom $\alpha$-Eisen-Wert (330 T) und belaufen sich auf 319,0(10)T bzw. 339,0(3)T. Es kommen nun 2 Effekte in Betracht, die diese Werte erklären könnten:

- Im (bct-) $\alpha^{\prime}$-Fe werden am Ort eines betrachteten Fe-Atoms von den nächsten Fe-Nachbarn (NN) folgende Felder erzeugt: 1. NN: 265(2)T , 2. NN: 342(2) T , 3. und 4. NN: 334(2) T [207]30|; in der Literatur weichen diese Werte jedoch oftmals untereinander ab (z.B. [208, 209]).

- Das HF-Feld des $\alpha$-Fe (33T) wird insbesondere von den substitutionellen Elementen $\mathrm{Cr}$ und $\mathrm{Ni}$ verändert [210]: Besitzt das Fe nur nächste Ni-Nachbarn, so beträgt das Feld 34,2 T, bei Ni- und Cr-Nachbarn hingegen 30,8 T [211].

Die entsprechenden CXM-Spektren sind ausnahmslos identisch zur Edelstahl-Referenzprobe, diese sind daher nicht dargestellt. Die strukturellen Umwandlungen laufen nur in oberflächennahen Schichten ab.

Das RBS-Spektrum (Abb. 6.25 zeigt die Referenz und die bei $200^{\circ} \mathrm{C}$ und bei $500^{\circ} \mathrm{C}$ angelassene Probe. Der Kohlenstoff-Anteil beträgt jeweils bei der nicht angelassenen Probe: $\approx 25(1)$ at. $\%, 200^{\circ} \mathrm{C}-$ Probe: $\approx 16(1)$ at. $\%$ und $500^{\circ} \mathrm{C}$-Probe: $\approx 4,8(10)$ at. $\%$. Dieser enorme thermisch aktivierte Abfall des Kohlenstoffanteils kann nur dadurch erklärt werden, dass dieser in tiefere Stahlschichten eindiffundiert und sich dem Nachweis durch RBS entzieht, der sich bis in eine Tiefe von ca. $500 \mathrm{~nm}$ erstreckt.

Auffällig ist der Teil des Spektrums an der Kante. Auf der niederenergetischen Seite bildet sich eine 12(3) nm breite Depression und darauf folgend eine „Nase“ aus, die ca. 9(3) nm dick ist und deren Yield höher ist als die Referenzprobe. Die Kante ist gegenüber dem Edelstahl zu niedriger Energie verschoben. Ein hoher Abteil an Chrom und Kohlenstoff kann die Kante im Spektrum verschieben. Die Nase kann mit einem sehr hohen Anteil von $>30$ at.\% Cr angefittet werden, doch sind die Anpassungen bzw. Simulationen mit WiNDF und RUMP in diesem Fall nicht eindeutig, da aufgrund der hohen Anzahl von 4 Elementen 
$\mathrm{Fe}, \mathrm{Cr}, \mathrm{Ni}, \mathrm{C}$ (Mo mit ca. 1,85 at.\% nicht berücksichtigt) jedes Profil durch geeignete Variationen des atomaren Element-Gehaltes angepasst werden kann, die nicht unbedingt realen Element-Verhältnissen entsprechen oder gar physikalisch möglich sind. 


\section{Kapitel 7}

\section{Diskussion zum Carburisierungs-Prozess}

\subsection{Phasenbildungs-Mechanismus im Eisen}

Die Ergebnisse zeigen, wie die Phasenbildung mit den Prozess-Parametern Pulszahl, Gasdruck und Energie zusammenhängen. Nur unter bestimmten Bedingungen werden reine Phasen erzeugt, die zusätzlich einen geringen Restanteil von maximal ca. 9 at.\% $\alpha$-Eisen enthalten:

- Energiedichte $H=4 \mathrm{~J} / \mathrm{cm}^{2}$ : Bei niedrigem Druck von $\mathrm{p}\left(\mathrm{CH}_{4}\right)=0,1$ bar und hoher Pulszahl $n>11 \times 12$. Energiedichte $H=3 \mathrm{~J} / \mathrm{cm}^{2}$ : Bei hohem Druck $\mathrm{p}\left(\mathrm{CH}_{4}\right)=4$ bar und maximaler Pulszahl von $n=16 \times 16$ bildet sich ausschließlich das $\varepsilon$-Carbid.

- Bei hohen Drücken $\mathrm{p}\left(\mathrm{CH}_{4}\right)>4$ bar und hohen Pulszahlen $n>11 \times 12$ entsteht das technologisch wichtige $\theta$-Carbid.

Die Bildung des Kohlenstoffs wird stets begleitet von der Bildung des $\varepsilon$-Carbids. Der umgekehrte Fall gilt jedoch nicht, da bei $3 \mathrm{~J} / \mathrm{cm}^{2}$ und $\mathrm{p}\left(\mathrm{CH}_{4}\right)=4$ bar $/ 16 \times 16$ Pulsen zwar $\varepsilon$-Carbid, jedoch kein Kohlenstoff entsteht. Bei hohem Druck wird selbst bei $33 \times 33$ Pulsen keine Kohlenstoff-Abscheidung beobachtet.

$\mathrm{Ab}$ welcher Temperatur sich Methan überhaupt zersetzt, darüber geben die thermodynamischen $\mathrm{Zu}$ standsgrößen des Methans Auskunft. Das Volumen bleibt während der Reaktion konstant, somit ist die geeignetete Zustandsgröße die Freie Reaktionsenergie F. Eine freiwillig ablaufende, exotherme Reaktion tritt dann ein, wenn $G>0$ [45].

$$
\Delta F=\Delta U-T \Delta S \quad, \quad \Delta U=\Delta H-p \Delta V \quad(p=101,3 \mathrm{kPa})
$$

Für die Reaktion $\mathrm{CH}_{4} \rightarrow \mathrm{C}+2 \mathrm{H}_{2}$ gilt mit den entsprechenden Werten $\Delta H=-74,85 \mathrm{~kJ} / \mathrm{mol}, \Delta S=80,96$ $\mathrm{J} /(\mathrm{mol} \cdot \mathrm{K})$ : F ür $T>651{ }^{\circ} \mathrm{C}$ ist $F>0$. Diese Temperatur wird im Laser-Prozess bei weitem übertroffen.

Von der konventionellen rf-Plasma-Erzeugung ist bekannt, dass bei sehr niedrigen Drücken der Größenordnung $\mathrm{p}=0,005$ bar (stoß-)ionisierte und angeregte Radikale wie $\mathrm{CH}_{4}^{+}, \mathrm{CH}_{3}^{+}, \mathrm{CH}_{2}^{+}$und $\mathrm{CH}^{+}$erzeugt werden 6 212]. Diese werden auf der Oberfläche adsorbiert und carburisieren das Substrat nach Dissoziation in Kohlenstoff und Wasserstoff. Das Methyl-Radikal ist dabei ein unerläßliches Zwischenprodukt.

Die Entstehung des Kohlenstoff-Fallouts hängt vom Gasdruck, Energiedichte und Laserspot-Überlapp zusammen. Die Pulsabfolge und die übrigen Parameter sind bei $4 \times 4$ - und der $11 \times 12$-Probe identisch. Die Frage ist nun, warum ein höherer Pulsüberlapp Kohlenstoff ausfallen lässt. Eine plausible Erklärung ist, dass bei niedrigsten Pulszahlen die Fläche schneller abgescannt wird und der Vorgang beendet ist, bevor ein merklicher Anteil gebildet werden kann. Bei hohen Pulszahlen $(11 \times 12$ und $16 \times 16)$ wird mehr Zeit beansprucht, um dieselbe Fläche zu mäandrieren. Bis zum Ende des Prozesses kann mehr Kohlenstoff freigesetzt werden.

Ein wichtiger Effekt, der hierbei offensichtlich eine Rolle spielt, ist das „plasma confinement“. Simulationen von Laser-Plasma-Dynamiken in einem Bereich von 1-100 bar zeigten, dass die Mechanismen der Plasma-Expansion sehr stark vom umgebenden Gasdruck abhängen [213] (Laserparameter: $I=10^{7}$ $10^{8} \mathrm{~W} / \mathrm{cm}^{2}, \lambda=1,06 \mu \mathrm{m}$ mit $\left.\tau_{P}=1 \mu \mathrm{s}\right)$. Je höher der Druck der Atmosphäre ist, desto dünner ist die sich ausbreitende Plasmaschicht. Wenn nun bei der Reaktion Kohlenstoff entsteht und sich mit dem 
ausdehnenden Plasma fortbewegt, so ist dessen Reichweite an die Ausdehnung des Plasmas gekoppelt. Ein niedriger Druck von typischerweise 0,1 bar verschiebt gemäß $\mathrm{CH}_{4} \rightarrow \mathrm{C}+2 \mathrm{H}_{2}$ das Gleichgewicht nach rechts, was zusätzlich zum plasma confinement die Kohlenstoff-Entwicklung erklären könnte. Mazhukins Laserparameter und die dieser Arbeit weichen zwar voneinander ab, doch kann man zumindest die Plasma-Dynamik qualitativ zur Erklärung einiger Beobachtungen heranziehen. Die Fläche und Kontaktzeit zwischen Probenoberfläche und dem aktiven Plasma steigt mit erhöhtem Druck an. Dadurch ist die Oberfläche komplett von weiterer Laserstrahlung abgeschirmt. Dieser ,shielding effect" bewirkt, dass das dichte Plasma den Kontakt zur Oberfläche verliert und somit deren direktes Aufheizen unterbunden wird. Das Thermo-Coupling 5.11 hat gezeigt, dass bei niedrigem Druck $(0,1$ bar $)$ die Durchschnitts-Temperatur höher ist als bei hohem Druck. Die Laser-Strahlung dringt im Fall niedrigen Druckes noch durch das Plasma hindurch, wodurch die höhere Oberflächentemperatur zustandekommt.

Einen weiteren wichtigen Ansatz liefert das LSC-LSD-Modell des Plasmadrucks (s. Abb. 4.5): Es wurde bereits festgestellt, dass eine Schwellenenergiedichte von mindestens $3,6 \mathrm{~J} / \mathrm{cm}^{2}$ vorhanden sein muss, um Kohlenstoff freizusetzen. Der Oberflächendruck $p_{S}$ liegt dann im LSD-Bereich bei ca. 4 MPa. Eine geringfügigge Verkleinerung der Energiedichte führt in den LSC-Bereich mit einem höheren Oberflächendruck, der das Reaktionsgleichgewicht nach links verschiebt bzw. zu einer erhöhten Auswirkung des plasma confinements führt.

Es ist etwas ungewöhnlich, unter welchen Voraussetzungen das $\varepsilon$-Carbids gebildet wird: Bei lediglich 3 $\mathrm{J} / \mathrm{cm}^{2}$ bildet es sich selbst bei hohen Drücken $\left(\mathrm{p}\left(\mathrm{CH}_{4}\right)=4\right.$ bar. Bei $4 \mathrm{~J} / \mathrm{cm}^{2}$ entsteht hingegen ausschließlich das $\theta$-Carbid. Die Doppel-Bestrahlungen haben weiterhin gezeigt, dass nur unter konstantem hohen Druck $\theta$-Carbid bei $4 \mathrm{~J} / \mathrm{cm}^{2}$ und $11 \times 12$ Pulsen entsteht.

Bei hohem Druck und unabhängig von der Pulszahl ist in keinem Fall eine Schwärzung zu verzeichnen. Falls sich Kohlenstoff bilden sollte, so wird dieser auf den Bereich des Plasmas, das nun einen viel geringeren Raum beansprucht, begrenzt und unmittelbar in die Oberfläche eingebracht. Aufgrund der höheren $\mathrm{CH}_{4}$-Dichte steht mehr Kohlenstoff zur Verfügung, der pro Laserpuls inkorporiert wird. Bei einer Messreihe, in der das Substrat mit Einzelpulsen $\left(\mathrm{H}=4 \mathrm{~J} / \mathrm{cm}^{2}\right)$ bestrahlt wurde, hat sich herausgestellt, dass bei $\mathrm{p}\left(\mathrm{CH}_{4}\right)=2$ bar die Kohlenstoffaufnahme beträchtlich höher ist als bei $\mathrm{p}\left(\mathrm{CH}_{4}\right)=0,5$ bar. Die Abb. 7.1 und 7.2 zeigen den Kohlenstoffgehalt bis in einer Tiefe von ca. $500 \mathrm{~nm}$. Der Gehalt ist hierbei in der allgemein üblichen RBS-Einheit angegeben. Der Methan-Druck beträgt hierbei 0,5 bar und 2 bar. Der

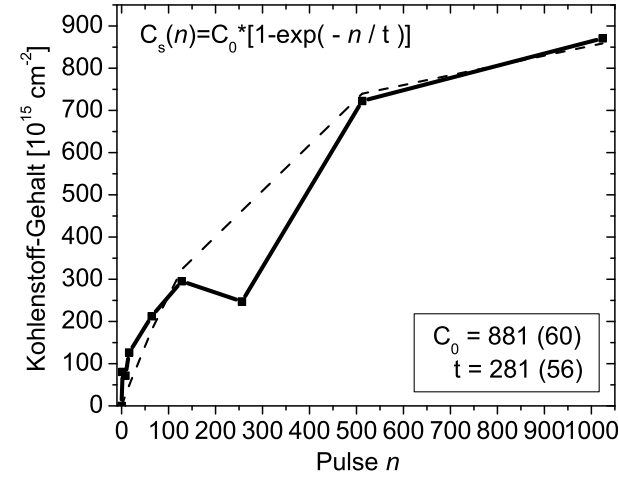

Abbildung 7.1: Kohlenstoffgehalt der Pulsserie bei $\mathrm{p}\left(\mathrm{CH}_{4}\right)=0,5$ bar.

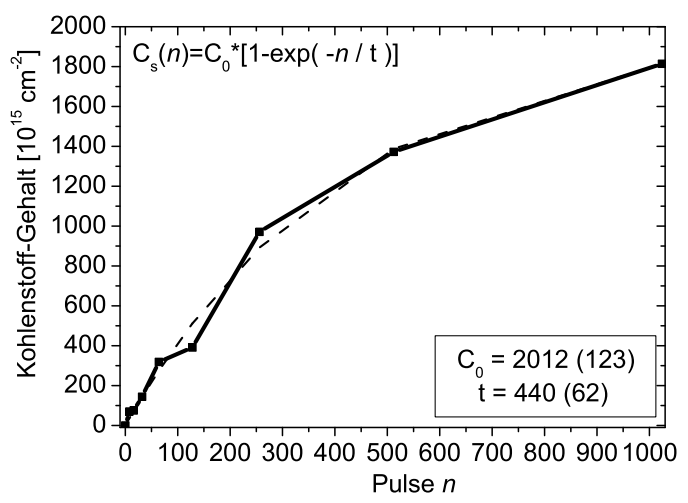

Abbildung 7.2: Kohlenstoffgehalt der Pulsserie bei $\mathrm{p}\left(\mathrm{CH}_{4}\right)=2$ bar.

Kohlenstoffgehalt ist erwartungsgemäß bei 1000 Pulsen maximal, und zwar bei $\mathrm{p}\left(\mathrm{CH}_{4}\right)=2$ bar höher als bei $\mathrm{p}\left(\mathrm{CH}_{4}\right)=0,5$ bar. In Abb. 7.3 ist dieser Sachverhalt noch einmal dargestellt. Zusätzlich ist der Gehalt der mäandrierten Probe bei $\mathrm{p}\left(\mathrm{CH}_{4}\right)=2$ bar und $33 \times 33$ Pulsen angegeben. Man erkennt, dass der Kohlenstoffgehalt der mäandrierten Probe (ca. 33 at.\%) geringer ist als der der Einzelpuls-Probe, der mit ca. 42 at.\% sehr hoch ist. Diese Beobachtung lässt sich auf alle Proben übertragen: Vergleicht man die mäandrierten Proben zwischen $4 \times 4$ und $33 \times 33$ bei $\mathrm{p}\left(\mathrm{CH}_{4}\right)=2 / 4$ bar mit den Einzelpuls-Proben zwischen 16 und 1000 Pulsen bei $\mathrm{p}\left(\mathrm{CH}_{4}\right)=2$ bar, so stellt man fest, dass der Kohlenstoffgehalt der Einzelpuls-Serie (trotz niedrigeren Druckes) zumindest gleich oder höher ist. Lediglich die $11 \times 12$ Probe weist als einzige einen höheren Kohlenstoffgehalt auf. (s. Abb.7.4). Die Gegenüberstellung der mit $4 \times 4$-Pulsen bestrahlten Proben (Abb. 5.28) zeigt ebenfalls, dass bei hohen Drücken am meisten Kohlenstoff eingebracht wird. Das 


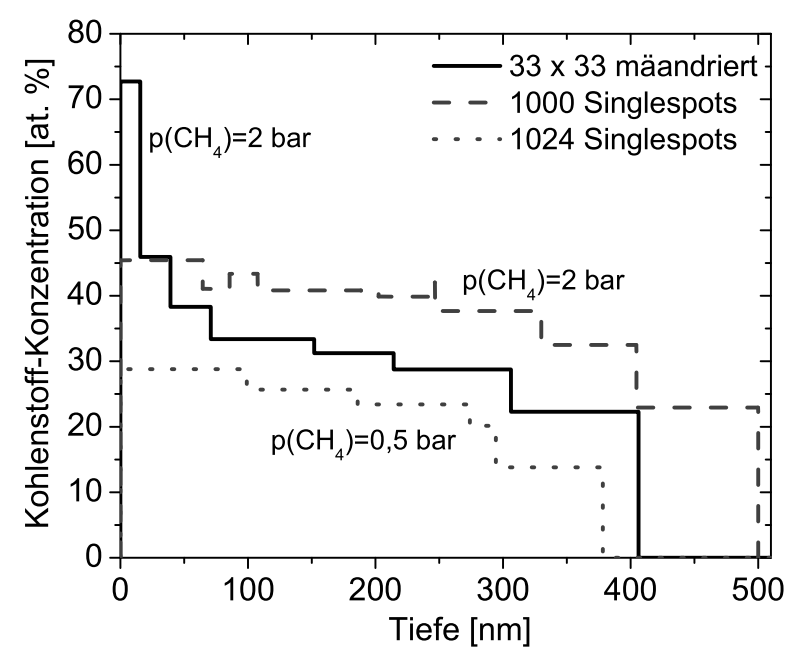

Abbildung 7.3: Vergleich der Proben, die jeweils mit 1000 Pulsen im Mäander- und Einzelpuls-Modus bestrahlt wurden. Der Druck beträgt $\mathrm{p}\left(\mathrm{CH}_{4}\right)=0,5$ bzw. 2 bar.

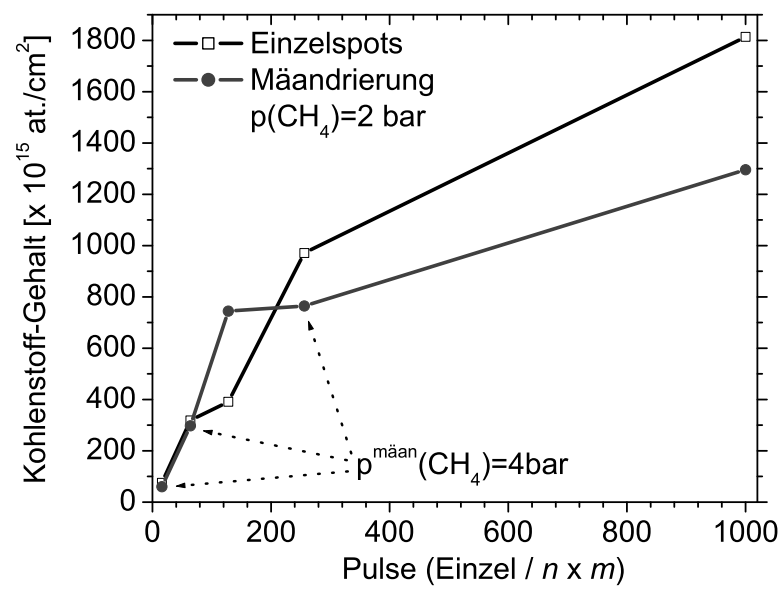

Abbildung 7.4: Kohlenstoffgehalt der Pulsserie bei $\mathrm{p}\left(\mathrm{CH}_{4}\right)=2$ bar.

Ausgangsmaterial besteht aus $\alpha$-Fe und $\gamma$ - $\mathrm{Fe}(\mathrm{C})$, dessen Gesamtanteil und Kohlenstoffgehalt mit steigendem Druck zunimmt. Zu Begin der Phasenbildungs-Sequenz bildet sich stets $\gamma-\mathrm{Fe}(\mathrm{C})$, das maximal ca. 12 at.\% Kohlenstoff aufnehmen kann. Weitere Kohlenstoffinkorporation führt dann je nach Gasdruck zur Bildung des $\varepsilon$-Carbids, dessen niedrigster Kohlenstoffgehalt bei ca. 14,3 at.\% liegt $\left(\mathrm{Fe}_{6} \mathrm{C}\right)$ und sich direkt dem $\gamma-\mathrm{Fe}(\mathrm{C})$ anschließt. Der Gehalt kann kontinuierlich bis auf maximal 33,3 at.\% $\left(\mathrm{Fe}_{2} \mathrm{C}\right)$ anwachsen. Die Hochdruckphase $\theta$-Carbid hingegen ist eine stöchiometrische Phase und bildet sich bei genau 25 at.\% C. Der Variationsbereich ist in diesem Fall also sehr schmal. Wie die Doppelbestrahlungen gezeigt haben (s. Kap. 5.10, ist der Druck der entscheidende Parameter, der erst zur Bildung des $\theta$-Carbides führt.

\subsection{Phasenbildungs-Mechanismus im Edelstahl}

Die markantesten Unterschiede zwischen der Phasenbildung des Eisens und Edelstahls sind im folgenden kurz aufgeführt:

- Die bestrahlten Edelstahlproben sowohl der Druck- als auch der Pulsserie zeigen im XRD-Spektrum jeweils Doppelpeaks, die vom fcc-Grundgitter und vom gedehnten Gitter des $\gamma$-Fe herrühren.

- Die XRD- und Mössbauer-Spektren der Edelstahl-Druckserie bei $11 \times 12$ Pulsen ähneln einander, während die Eisenproben unterschiedliche Phasen aufweisen.

- Die Edelstahl-Pulsserie bei $\mathrm{p}\left(\mathrm{CH}_{4}\right)=1$ bar weist ab $8 \times 8$ Pulsen im XRD-Spektrum einen Untergrund auf, der auf eine amorphe Phase hindeutet. Die kristalline Phase bildet hierbei nur interstitiell eingelagerter Kohlenstoff im fcc-Gitter (als $\gamma$ - $\mathrm{Fe}(\mathrm{C})$ ), es ist jedoch keine stöchiometrische Phase präsent. Erst ab $14 \times 14$ Pulsen bilden sich kristalline Carbid-Phasen. Die amorphe Phase tritt hingegen bei den Eisenproben nicht auf.

Die Sequenz der Phasenbildung mit steigendem Kohlenstoffeintrag im Edelstahl lässt sich wie folgt darstellen:

Grundaustenit $\rightarrow$ Expanded / Grundaustenit $\rightarrow$ Expanded / Grundaustenit plus amorphe Phase $\rightarrow$ kristalline Phasen, Carbide

Die amorphe Phase wird jedoch weiterhin von einer kristallinen Phase begleitet: Der Doppelpeak des retained und des expanded austenite ist sowohl an der Oberfläche (ca. $170 \mathrm{~nm}$, entspricht $1^{\circ}$ grazing incidence bei XRD) als auch in ca. $810 \mathrm{~nm}\left(5^{\circ}\right.$ g.i. $)$ nachzuweisen. Es bilden sich erst ab $14 \times 14$ Pulsen 
(Fe,Cr,Ni)-Carbide. Bei $14 \times 14$ Pulsen ist noch der amorphe Untergrund erkennbar, der bei $16 \times 16$ Pulsen verschwunden ist. Diese amorphen Phasen, die sog. metallischen Gläser oder metallic glassy alloys, entstehen, wenn folgende Bedingungen erfüllt sind [214,|215]:

1) Es müssen in der flüssigen Legierung sehr hohe Abkühlraten $\dot{T}$ erreicht werden. In diesem Fall wird die Keimbildung unterdrückt, die zur Kristallisation führt; die Flüssigkeit bleibt „unterkühlt“. Bei Verwendung eines Lasers kann leicht $\dot{T} \approx 10^{12} \mathrm{~K} / \mathrm{s}$ erreicht werden [216]. Die Abkühlrate, die bei diesen Experimenten erreicht wurden, beträgt einige $10^{10} \mathrm{~K} / \mathrm{s}$.

Die Bestrahlung der Eisenproben führt ebenfalls zu hohen Abkühlraten, jedoch zu keiner Formation der amorphen Phase. Weiterhin verschwindet bei $16 \times 16$ Pulsen die amorphe Phase. Da sich bei der Puls-Serie nur der Spottüberlapp ändert und jeder Puls gleiche Parameter besitzt sowie identische Abkühlraten zur Folge hat. Die Bildung der amorphen Phase ist demzufolge auf den Kohlenstoffeintrag in das Material zurückzuführen.

2) Das Atomradien-Verhältnis der Legierungselemente spielt eine große Rolle [217]. Dieses Verhältnis führt zu einer bestimmten kritischen Konzentration der gelösten Substanz (hier Kohlenstoff).

In weiteren ähnlichen Modellen [218, 219] werden Bedingungen aufgeführt, unter denen der glasartige Zustand stabilisiert wird: Packt man ungeordnet große Metallatome zusammen, so entstehen Strukturen, in deren Hohlräume kleinere Nichtmetall-Atome $N$ hineinpassen. Für die Zusammensetzung ergibt sich $\mathrm{M}_{80} \mathrm{~N}_{20}$. Tatsächlich existieren viele solcher Gläser mit ca. 20 at.\% Metalloid-Anteil, die bei Raumtemperatur stabil sind. Diese Modelle treffen hier für den Edelstahl nicht zu. Die amorphe Phase entsteht bereits bei niedrigeren Konzentrationen. Der gesamte Kohlenstoffgehalt der überwiegend amorphen Proben beträgt in der $11 \times 12$-Probe ca. 25 at.\%, davon sind in der amorphen Phase maximal ca. 16,3 at.\% enthalten. Erhöhung des Kohlenstoffgehalts führt indes zur Bildung kristalliner Phasen $(14 \times 14$ - und 16×16-Proben).

Der entscheidende Punkt der Amorphisierung ist, dass die legierenden Elemente die kristalline Phase aufgrund des Größen-Effektes destabilisieren und nicht den flüssigen Zustand stabilisieren [215]. Um eine feste Lösung zu destabilisieren, ist es erforderlich, eine Volumenausdehnung der metallischen Matrix um 6-8\% herbeizuführen [217]. Die kritische Konzentration $c_{\text {krit }}$, oberhalb der die kritalline Phase instabil wird, errechnet sich zu:

$$
c_{\mathrm{krit}}=0,1 \cdot \frac{V_{M}}{V_{M}-V_{N}},
$$

$V_{M}$ und $V_{N}$ sind die Atomvolumina des Metalls bzw. des Nichtmetalls. Mit den Atomradien $R_{\mathrm{Fe}}=0,126 \mathrm{~nm}$ und $R_{\mathrm{C}}=0,077 \mathrm{~nm}$ folgt:

$$
c_{\mathrm{krit}}=0,1 \cdot \frac{R_{\mathrm{Fe}}^{3}}{R_{\mathrm{Fe}}^{3}-R_{\mathrm{C}}^{3}} \simeq 0,13
$$

In Abb. 7.5 ist der Kohlenstoff, der nur in der amorphen Phase enthalten ist, gegen die Pulszahl aufgetragen. Dieser Anteil ergibt sich, wenn von dem Gesamtanteil, der durch RBS bestimmt wurde, der Anteil abgezogen wird, der in der kristallinen Phase $(\gamma-\mathrm{Fe}(\mathrm{C}))$ enthalten ist. Dieser Anteil wurde aus CEMS- und XRD-Messungen bestimmt und für die Auftragung gemittelt. Gemäß XRD-Spektrum (s. Abb. 6.2 erscheint die amorphe Phase relativ schwach bei $8 \times 8$ Pulsen, ist jedoch richtig ausgeprägt bei $10 \times 10 / 11 \times 12$, was gut mit dem kritischen Wert $c_{\text {krit }} \approx 13$ at.\% übereinstimmt (s. Abb. 7.5).

Eine Aussage über die kritische Abkühlrate $\dot{T}$ bzw. über die Nukleationsrate, die den Kristallisationsprozess steuert, kann unter Zuhilfenahme der Nukleationstheorie getroffen werden. Die Aktivierungsenergie der Nukleation $\Delta G$ ist gegeben durch [215]:

$$
\Delta G=\frac{16 \pi \gamma^{3}}{3 G_{V}^{2}} \propto \frac{\gamma^{3}}{(\Delta T)^{2}},
$$

wobei $\gamma$ die Freie Energie pro Einheits-Grenzfläche in der Fest-Flüssig-Grenzschicht, $G_{V}$ die Freie Energie pro Einheitvolumen, die die treibende Kraft zur Flüssig-Fest-Umwandlung darstellt und $\Delta T=T_{\text {schm }}-T$ mit der Schmelztemperatur $T_{\text {schm. }}$. Die Dichte der stabilen Kristallisationskeime (sog. nuclei) ist gegeben durch [3]:

$$
n \propto \exp \left(\frac{\Delta G}{k T}\right)
$$




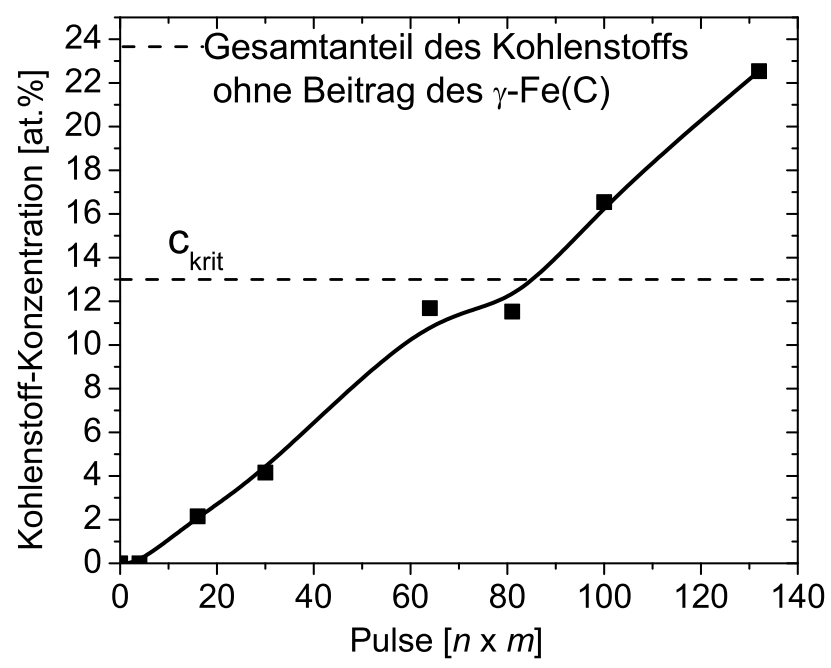

Abbildung 7.5: Kohlenstoffanteil der amorphen Phase in Abhägigkeit der Pulszahl. Die kritische Konzentration ist eingezeichnet.

Die Größe $n$ hängt nun mit der Abkühlrate $\dot{T}$ und der Nukleationsrate $\nu_{D}$ über

$$
n \propto \nu_{D} \cdot \dot{T}
$$

zusammen. Für die Diffusivität $D(T)$ gilt [3]:

$$
D(T)=D_{0} \cdot\left(\frac{Q_{D}}{k T}\right)
$$

dabei ist $Q_{D}$ die Diffusions-Aktivierungsenergie. Die Kühlrate und die direkt mit der Diffusivität $D$ verknüpft. Die Nukleationfrequenz $\nu_{D}$ gibt an, wie oft die Atome in der flüssigen Phase an den Nucleati-

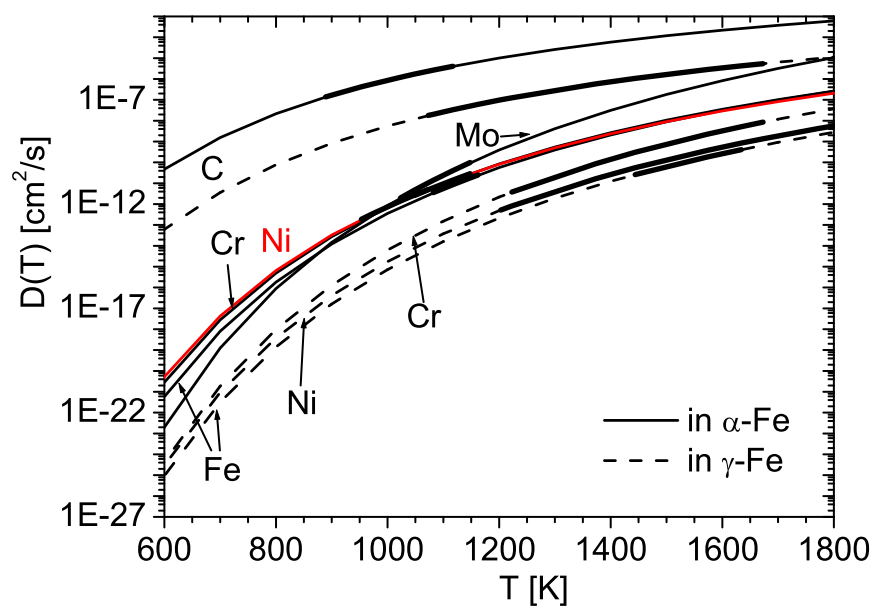

Abbildung 7.6: Diffusivität $D(T)$ der Elemente Fe, Ni, Cr und C in $\alpha$ - und $\gamma$-Fe. Die dick abgesetzten Linien stellen die reproduzierten, experimentellen Daten nach [220] dar.

onskeimen der festen Phase in Kontakt geraten [3]:

$$
\nu_{D} \propto D(T) \cdot\left(\frac{\Delta G}{k T}\right)
$$

In Abb. 7.6 sind die Diffusivitäten der Elemente $\mathrm{Fe}, \mathrm{Cr}, \mathrm{Ni}, \mathrm{Mo}$ (nur $\alpha$ ) und $\mathrm{C}$ in $\alpha$ - und $\gamma$-Fe dargestellt. Ein wesentliches Merkmal ist, dass die Diffusivität in der fcc-Matrix $(\gamma-\mathrm{Fe})$ um 2-3 Größenordnungen geringer ist als in der bcc-Matrix $(\alpha$-Fe). Die Nukleationsrate in der fcc-Matrix (Gl. 7.8) wird dementsprechend geringer sein. Daher sollte eine um 2-3 Größenordnung höhere Abkühlrate erforderlich sein, um die bcc-Matrix zu amorphisieren. 
Diese Argumente sind zwar sehr qualitativ, können aber eine Erklärung dafür liefern, warum es bei ähnlichen Kohlenstoff-Konzentrationen und gleichen Laser-Parametern bzw. Bedingungen in den $\alpha$-EisenProben im Gegensatz zu den Edelstahl-Proben zu keiner merklichen Ausbildung einer amorphen Struktur kommt.

Eine interessante Analogie zur Metalloid-Ionen-Implantation kann hier gezogen werden, die ebenfalls ein Nicht-Gleichgewichtsprozess ist: Hohmuth et al. [8] bestrahlten Eisen mit $\mathrm{B}^{+}, \mathrm{N}^{+}-$und $\mathrm{C}^{+}$-Ionen. Auf diese Weise bildeten sich kristalline und amorphe Phasen. Die Resultate zeigten, dass nur $\mathrm{C}^{+}$ (und $\mathrm{B}^{+}$) ) Ionen die Bildung amorpher Phasen herbeiführen konnten, $\mathrm{N}^{+}$-Ionen hingegen nicht. Damit amorphe Metall-Nichtmetall-Legierung überhaupt entstehen, muss das Radienverhältnis von Metall $\left(R_{\mathrm{M}}\right)$ und Nicht-Metall $\left(R_{\mathrm{N}}\right)$ der Bedingung $0,59<R_{\mathrm{N}} / R_{\mathrm{M}}<0,88$ genügen (Hägg-Regel), für Fe-C ist das $R_{\mathrm{C}} / R_{\mathrm{Fe}}=0,611$. Nach der obigen Ausführung muss eine höhere Abkühlrate erreicht worden sein. Nach [7] errechneten einige Autoren, dass die Abkühlrate bei der Ionenimplantation bis zu $\dot{T} \sim 10^{15} \mathrm{~K} / \mathrm{s}$ betragen kann. Der letzte Punkt betrifft die neben der amorphen Phase auftretenden Doppelpeaks. Offenbar wird der Kohlenstoff bis zur Sättigungsgrenze von ca. 9,0 at.\% in das $\gamma$-Fe(C) eingebracht. Weitere Kohlenstoffinkorporation erhöht nur den Gehalt in der amorphen Phase, ohne dass der Anteil der kristallinen $\gamma$-Phase zugunsten der amorphen Phase abnimmt.

Für ein Cu-Ti-System wurde bei ähnlichen Abkühlraten von $\dot{T} \sim 10^{10} \mathrm{~K} / \mathrm{s}$ gefunden, dass die Ausbildung der fcc-Struktur als einzige mit der Glas-Bildung konkurrieren kann und daher gemeinsam mit ihr auftritt [221]. Einer Erkärung dafür liegt die Idee zugrunde, dass fcc eine dichtest-gepackte Struktur ist, die der ungeordneten Atomanordung in der Flüssigkeit am meisten ähnelt. Wird die KohlenstoffKonzentration weiter bis auf ca. 26 at.\% gesteigert $(16 \times 16$-Probe), so wird die Bedingung nicht mehr erfüllt, die amorphe Phase, die bis zu einer Konzentration von 20 at.\% C aufrecht erhalten werden kann, zu formieren und zu stabilisieren. Stattdessen bilden sich ausschließlich kristalline Phasen in Form von Carbiden. 


\section{Kapitel 8}

\section{Zusammenfassung}

Im Rahmen dieser Arbeit sind die Untersuchungen zur Bestrahlungen von Eisen und Edelstahl in reaktiver Atmosphäre (hauptsächlich Methan) durchgeführt worden. Das Hauptinteresse liegt darin, unter welchen Bedingungen welche Anteile an verschiedenen Fe-C-Verbindungen entstehen. Dabei wird untersucht, wie die Laser-Parameter Druck, Pulsanzahl und Energie die Phasenbildung im Material beeinflussen. Im folgenden werden die Ergebnisse der Eisen- und Stahlproben zusammengefasst.

\subsection{Eisen}

Die Kohlenstoffaufnahme, die Phasenbildung und der entsprechende Phasenanteil im Eisen hängt empfindlich von den Prozess-Parametern Gasdruck, Pulszahl und Energie ab. Weiterhin beeinflusst auch die Gasmischung die Entstehung einer bestimmten Phase: Die Zugabe eines inerten Gases ändert die Phasenzusammensetzung beträchtlich bei ansonsten gleichen Verhältnissen. Variation aller Laserparameter in den Grenzen für Druck: 0,1 bar $\leq \mathrm{p}\left(\mathrm{CH}_{4}\right) \leq 10$ bar, Pulszahl: $4 \times 4 \leq n \leq 16 \times 16$ und Energiedichte: 3,25 $\mathrm{J} / \mathrm{cm}^{2} \leq H \leq 6,9 \mathrm{~J} / \mathrm{cm}^{2}$ führen zu einer Vielfalt an Phasen und Phasengemischen aus $\gamma$-Fe(C), $\alpha$-Fe, $\varepsilon$ und $\theta$-Carbid:

Als instruktives Beispiel sei erwähnt, dass bei Bestrahlung in Atmosphären mit $\mathrm{p}\left(\mathrm{CH}_{4}\right) \geq 4$ bar, Pulszahlen $n \geq 11 \times 12$ und Energiedichten $H=4 \mathrm{~J} / \mathrm{cm}^{2}$ das harte, orthorhombische $\theta-\mathrm{Fe}_{3} \mathrm{C}$ (Zementit) entsteht, welches den höchsten Härtegrad aller gebildeten Phasen aufweist. Wird stattdessen die Bestrahlung unter einem Druck von $\mathrm{p}\left(\mathrm{CH}_{4}\right)=0,1$ bar durchgeführt, so bildet sich das hexagonale $\varepsilon$ - $\mathrm{Fe}_{x} \mathrm{C}$, das relativ weich ist.

Die Ergebnisse zeigen außerdem, dass bei den niedrigsten Drücken im Gegensatz zu sehr hohen Drücken Kohlenstoff ausfällt, was auf eine unterschiedliche Ausdehnug des Plasma schließen läßt.

\subsection{Edelstahl}

Die erste Serie des Edelstahl wurde unter einem Druck von $\mathrm{p}\left(\mathrm{CH}_{4}\right)=1$ bar und mit einer Energiedichte von $H=3,7 \mathrm{~J} / \mathrm{cm}^{2}$ im Bereich von $2 \times 2 \leq n \leq 16 \times 16$ Pulsen bestrahlt. Es zeigt sich, dass sich anders als im Eisen zunächst kein Carbid, sondern eine kristalline Fe-C-Phase bildet, deren Kohlenstoffgehalt mit steigender Pulszahl bis zu einer Sättigungsgrenze zunimmt. Diese Phase besteht zum einen aus dem Grundgitter des Edelstahls (Grund-Austenit) und zum anderen aus Austenit, dessen Gitter durch den Kohlenstoff aufgeweitet ist (expanded austenite).

Interessant ist, dass sich ab einer Konzentration von ca. 13 at.\% Kohlenstoff zusätzlich eine ausgeprägte magnetische, amorphe Phase bildet. Erst bei der höchsten Anzahl von $16 \times 16$ Pulsen verschwindet die amorphe Phase und es kommt zur Ausbildung vieler Carbide. Die fcc-Struktur läßst sich demnach leichter amorphisieren als die bcc-Struktur.

Die zweite Serie wurde bei $11 \times 12$ Pulsen und einer Energiedichte $H=4 \mathrm{~J} / \mathrm{cm}^{2}$ im Bereich von 0,1 bar $\leq \mathrm{p}\left(\mathrm{CH}_{4}\right) \leq 4$ bar bestrahlt. Während beim Eisen die Phasenbildung stark druckabhängig ist, ändert sich beim Edelstahl die Phasenzusammensetzung kaum merklich; es ist durchgängig die amorphe Phase mit dem Austenit präsent. 



\section{Kapitel 9}

\section{Ausblick}

Ein Schwerpunkt zukünftiger Untersuchungen wird es sein, die Ergebnisse dieser Arbeit mit KohlenstoffTiefenprofilen zu ergänzen und die Diffusionsvorgänge mit theoretischen Vorhersagen zu vergleichen, wie es im Fall der Laser-Nitrierung durchgeführt worden ist [177, 24, 163]. Die Resonanzenergie des Kohlenstoffisotops ${ }^{15} \mathrm{C}$ für resonante Kernreaktionsanalyse (RNRA) liegt bei $1748 \mathrm{keV}$ [222] und liegt somit außerhalb des IONAS-Energiebereichs.

Weiterhin ist es für die Laser-Plasma-Verfahren sehr aufschlussreich, die verschiedenen Ionenarten der reaktiven Gasatmosphäre durch spektroskopische Verfahren zu identifizieren. Zusammen mit weiteren Thermo-Kopplungs-Experimenten, die unter variablen Prozessbedingungen wie Energie, Druck und Pulsanzahl durchgeführt werden, könnten durch die Untersuchung der Art und Menge der Ionenspezies tiefere Einblicke in den Carburisierungs-Mechansimus bzw. spezifischen Phasenbildungsprozess gewonnen werden.

Die Messungen haben gezeigt, dass die Gasmischung $\mathrm{Ar} / \mathrm{CH}_{4}$ zur Bildung anderer Phasen führten als die jeweils reinen Gase. Durch weitere Serien mit Edelgasbeimischungen kann festgestellt werden, ob nicht viel niedrigere Werte der Prozess-Parameter (Druck, Energie und Pulszahl) ausreichen, um dasselbe Resultat zu erzielen wie bei den bisherigen hohen Werten. 



\section{Literaturverzeichnis}

[1] H.J. Grabke, Materiali in Tehnoloije 36 (2002) 297.

[2] J. Zhang and O. Ostrovski, ISIJ Int. 41 (2001) 333.

[3] W.D. Callister, Materials Science and Engineering: An Introduction, 6 ed. (Wiley, New York, 2003).

[4] J.M. Baek et al., Surf. Coat. Techn. 131 (2000) 568.

[5] M. Okumiya et al., Surf. Coat. Techn. 174 (2003) 1171.

[6] I. Jauberteau et al., J. Vac. Sci. Technol. A 18 (2000) 108.

[7] D.M. Follstaedt, Nucl. Instr. and Meth. B 7/8 (1985) 11.

[8] K. Hohmuth et al., Nucl. Instr. and Meth. 209/210 (1983) 249.

[9] A. Königer et al., Phys. Rev. B 80 (1997) 8143.

[10] A.M.C. Pérez-Martin et al., Mat. Sci. Eng. B 19 (1993) 281.

[11] D. Bäuerle, Laser Processing and Chemistry (Springer-Verlag, Berlin, Heidelberg, 2000).

[12] W.M. Steen, Laser Material Processing (Springer-Verlag, Berlin, Heidelberg, New York, 1998).

[13] M. van Allmen and A. Blatter, Laser-Beam Interactions with Materials, Springer Series in Material Science (Springer-Verlag, Berlin, 1986).

[14] T.R. Jervis, M. Nastasi and J.P. Hirvonen, Mat. Res. Soc. Symp. Proc. 279 (1993) 665.

[15] J.C. Ion, K.E. Easterling and M.F. Ashby, Acta Metall. 32 (1984) 1949.

[16] M.F. Ashby and K.E. Easterling, Acta Metall. 32 (1984) 1935.

[17] J.C. Ion, H.R. Shercliff and M.F. Ashby, Acta Metall. Mater. 40 (1992) 1539.

[18] F. Dausinger and J. Shen, ISIJ Int. 33 (1993) 925.

[19] M. Hegelich, Phys. Rev. Lett. 89 (2002) 085002.

[20] M. Roth, Physik J. 3 (2004) 31.

[21] P. Canova and E. Ramous, J. Mat. Sci. 21 (1986) 2143.

[22] M. Tayal and K. Mukherjee, Mat. Sci. Eng. A 174 (1994) 231.

[23] E. Carpene and P. Schaaf, Appl. Phys. Lett. 80 (2002) 891.

[24] E. Carpene, Dissertation, Universität Göttingen, 2002.

[25] V.N. Anisimov et al., Phys. Chem. Mech. Surfaces 3 (1985) 2756.

[26] I.N. Mihailescu et al., J. Appl. Phys. 75 (1994) 5286. 
[27] H.O.T.B. Massalski, P.R. Subramanian and L. Kacprzak, editors, Binary Alloy Phase Diagrams (ASM International, Ohio, 1996).

[28] D.H. Jack and K.H. Jack, Mater. Sci. Eng. 11 (1973) 1.

[29] R.C. Ruhl and M. Cohen, Trans. AIME 245 (1969) 241.

[30] M. Ron, Iron-Carbon and Iron-Nitrogen Systems, Applications of Mössbauer Spectroscopy II, edited by R.L. Cohen, pp. 329-392, Academic Press, New York, 1980.

[31] F.P. Pickering, Constitution and Properties of Steels, Materials Science and Technology, edited by R.W. Cahn, P. Haasen and E.J. Kramer, volume 7, VCH, Weinheim, New York, Basel, Cambridge, 1987.

[32] J.M. Dubois and G.L. Caër, Acta Metall. 25 (1977) 609.

[33] M. Ron and Z. Mathalone, Phys. Rev. B 4 (1971) 774.

[34] Powder Diffraction File Database PDF-2, Newton Square, 1997, International Centre for Diffraction Data.

[35] R.C. Weast and M.J. Astle, Handbook of Chemistry and Physics (CRC Press, Boca Raton, 1983).

[36] Z. Mathalone et al., J. Appl. Phys. 42 (1971) 687.

[37] H. Jehn et al., Gases and Carbon in Metals. Part XIV: Ferrous Metals(2), Iron-Carbon, Physik Daten - Physics Data No. 5-14 (Fachinformations Zentrum, Karlsruhe, 1981).

[38] H. Bester and K.W. Lange, Arch. Eisenhüttenwesen 43 (1972) 283.

[39] G.G. Tibbetts, J. Appl. Phys. 51 (1980) 4813.

[40] D.C. Parris and R.B. McLellan, Acta. Met. 24 (1976) 523.

[41] D.E. Jiang, Phys. Rev. B 67 (2003) 214103.

[42] R.A. McKee, Phys. Rev. B 22 (1980) 2649.

[43] R.J. Meyer, E.H.E. Pietsch and A. Kotowski, Gmelin-Durrer: Metallurgie des Eisens - Theorie der Stahlerzeugung 1, Gmelins Handbuch der anorganischen Chemie, edited by H. Trenkler and W. Krieger, volume 5a, Springer, Berlin, Heidelberg, New York, 1978.

[44] J. Kunze, Nitrogen and Carbon in Iron and Steel (Akademie-Verlag, Berlin, 1990).

[45] C.E. Mortimer, Basiswissen der Chemie (Verlag Thieme, Stuttgart, 2003).

[46] G.D. Considine, Van Nostrand's Encyclopedia of Chemistry, 5 ed. (Wiley-Interscience, Hoboken, New Jersey, 2005).

[47] R.C. Ruhl and M. Cohen, Acta Met. 15 (1967) 159.

[48] D.L. Williamson, S. Bukshpan and R. Ingalls, Phys. Rev. B 6 (1972) 4194.

[49] S. Nagakura, J. Phys. Soc. Japan 14 (1959) 186.

[50] E.J. Fasiska and G.A. Jeffrey, Acta. Cryst. 19 (1965) 463.

[51] S. Nagakura, T. Suzuki and M. Kusunoki, Trans. JIM 22 (1981) 699.

[52] W.C. Chiou and E.A. Carter, Surf. Sci. 530 (2003) 87.

[53] J. Häglund, G. Grimvall and T. Jarlborg, Phys. Rev. B 44 (1991) 2914.

[54] G.B. Raupp and W.N. Delgass, J. Catal. 58 (1979) 348. 
[55] Y.V. Maksimov et al., Kinet. Catal. 15 (1974) 1144.

[56] G.L. Caër et al., J. Phys. Chem. 86 (1982) 4799.

[57] J.W. Niemantsverdriet and A.M. van der Kraan, J. Phys. Chem. 84 (1980) 3363.

[58] J.A. Amelse, J.B. Butt and L.H. Schwartz, J. Phys. Chem. 82 (1978) 558.

[59] R.A. Arents et al., Phys. Met. Metall. 36 (1973) 46.

[60] A.A. Zhukov et al., Acta Met. 21 (1973) 195.

[61] M. Ron, H. Shechter and S. Niedzwiedz, J. Appl. Phys. 39 (1968) 265.

[62] J.R.G. jr. and G. Parravano, J. Phys. Chem. 82 (1978) 1040.

[63] H. Ino et al., J. Phys. Soc. Jpn. 25 (1968) 88.

[64] K.W. Andrews, Acta Met. 11 (1963) 939.

[65] K.W. Andrews, Acta Met. 12 (1964) 921.

[66] F. Habashi, Alloys (Wiley-VCH, Weinheim, New York, 1998).

[67] C.W. Wegst, Stahlschlüssel - Key to Steel (Verlag Stahlschlüssel Wegst GmbH, Marbach, 1989).

[68] P. Haasen, Physical Metallurgy (Cambridge University Press, Cambridge, 1996).

[69] J.K.L. Lai et al., Mat. Sci. Eng. A 379 (2004) 308.

[70] ASM Handbook of Alloy Phase Diagrams, zitiert auf: http://www.sv.vt.edu/classes/ MSE2094_NoteBook/96ClassProj/experimental/ternary2.html.

[71] E. Ignatowitz, Werkstofftechnik für Metallbauberufe (Verlag Europa-Lehrmittel, Haan Gruiten, 2003).

[72] R.L. Mössbauer, Naturwissenschaften 45 (1958) 538.

[73] R.L. Mössbauer, Z. Physik 151 (1958) 124.

[74] R.L. Mössbauer, Science 137 (1962) 731.

[75] S.J. Campbell, W.A. Kaczmarek and M. Hoffmann, Hyp. Int. 126 (2000) 175.

[76] D.C. Cook, Hyp. Int. 141/142 (2002) 21.

[77] U. Gonser, editor, Mössbauer Spectroscopy, volume 5 of Topics in Applied Physics (Springer-Verlag, Berlin, Heidelberg, New York, 1975).

[78] U. Gonser, editor, Mössbauer Spectroscopy II, volume 25 of Topics in Current Physics (SpringerVerlag, Berlin, Heidelberg, New York, 1981).

[79] U. Gonser and R. Preston, Mössbauer Spectroscopy Applied in Amorphous Metals, Glassy Metals II, edited by H. Beck and H.J. Güntherodt, volume 53 of Topics in Applied Physics, chap. 4, p. 93, Springer-Verlag, Berlin, Heidelberg, New York, Tokyo, 1983.

[80] P. Gütlich, R. Link and A. Trautwein, Mössbauer Spectroscopy and Transition Metal Chemistry (Springer-Verlag, Berlin, Heidelberg, New York, 1978).

[81] L. May, An Introduction to Mössbauer Spectroscopy (Plenum Press, New York, 1971).

[82] U. Gonser, Mater. Sci. Eng. 3 (1968/69) 1.

[83] N.N. Greenwood and T.C. Gibb, Mössbauer Spectroscopy (Chapman and Hall, London, 1971). 
[84] G.N. Belozerski, Mössbauer Studies of Surface Layers, volume 81 of Studies in Physical and Theoretical Chemistry (Elsevier, Amsterdam, London, New York, Tokyo, 1993).

[85] G. Musiol et al., Kern- und Elementarteilchenphysik (Verlag Harri Deutsch, Frankfurt a.M., 1995).

[86] J.R. Tesmer and M. Nastasi, editors, Handbook of Modern Ion Beam Materials Analysis (MRS, Pennsylvania, 1995).

[87] F.J. Litterst et al., J. Phys. C 12 (1979) 5551.

[88] V.I. Goldanskii and R.H. Herber, Chemical Applications of Mössbauer Spectroscopy (Academic Press, New York and London, 1968).

[89] N. Ashcroft and N.D. Mermin, Festkörperphysik (Oldenbourg Verlag, München, Wien, 2001).

[90] K. Kopitzki, Einführung in die Festkörperphysik (Teubner, Stuttgart, 1993).

[91] H. Wegener, Der Mössbauer Effekt und seine Anwendungen in Physik und Chemie (Bibliographisches Institut, Mannheim, 1964).

[92] D.G. Rancourt, Nucl. Inst. Meth. B 44 (1989) 199.

[93] G. Schatz and A. Weidinger, Nukleare Festkörperphysik (Teubner, Stuttgart, 1992).

[94] D. Kamke, Einführung in die Kernphysik (Vieweg Verlag, Braunschweig, 1979).

[95] W. Greiner, Klassische ElektrodynamikTheoretische Physik (Verlag Harri Deutsch, Frankfurt a.M., 1991).

[96] A. Vértes, L. Korecz and K. Burger, Mössbauer Spectroscopy, volume 5 of Studies in Physical and Theoretical Chemistry (Elsevier, Amsterdam, Oxford, New York, 1979).

[97] W. Meisel, Hyp. Int. 45 (1989) 73.

[98] P. Schaaf, Dissertation, Universität Saarbrücken, 1991.

[99] P. Schaaf et al., Nucl. Instr. and Meth. B 53 (1991) 184.

[100] L. Blaes et al., Hyperfine Interactions 29 (1986) 1571.

[101] P. Schaaf et al., Hyperfine Interactions 92 (1994) 1189.

[102] F. Landry and P. Schaaf, GöMOSS - Windows Fitprogram for the Analysis of Mössbauer Spectra, unpublished, 1998.

[103] F.E. Wagner, J. de Physique 37 (1976) C6 673.

[104] P. Schaaf et al., Hyperfine Interactions 95 (1995) 199.

[105] C. Kittel, Einführung in die Festkörperphysik (Oldenbourg Verlag, München, Wien, 2002).

[106] A. Authier, Dynamical Theory of X-Ray Diffraction (Oxford University Press, Oxford, 2004).

[107] J. Als-Nielsen and D. McMorrow, Elements of Modern X-Ray Physics (Wiley, New York, 2001).

[108] B.G. Cullity and S.R. Stock, Elements of X-Ray Diffraction, 3 ed. (Prentice Hall, New Jersey, 2001).

[109] C. Suryanarayana and M.G. Norton, X-Ray Diffraction: A Practical Approach (Plenum Press, New York, London, 1998).

[110] E. Lifshin, X-Ray Characterization of Materials (VCH, Weinheim, 1999).

[111] J. Wong, EXAFS Studies of Metallic Glasses, Glassy Metals I, edited by H.J. Güntherodt and H. Beck, volume 42 of Topics in Applied Physics, Springer, Berlin, Heidelberg, New York, 1981. 
[112] B.K. Teo, EXAFS: Basic Principles and Data Analysis, volume 9 of Inorganic Chemistry Concepts (Springer-Verlag, Berlin, Heidelberg, New York, Tokyo, 1986).

[113] E.A. Stern, Phys. Rev. B 10 (1974) 3027.

[114] P.A. Lee, Phys. Rev. B 11 (1975) 2795.

[115] E.A. Stern, D.E. Sayers and F.W. Lytle, Phys. Rev. B 11 (1975) 4836.

[116] D.E. Sayers and E.A. Stern, Phys. Rev. Lett. 27 (1971) 1204.

[117] D.C. Koningsberger and R. Prins, X-Ray Absorption: Principles, Applications, Techniques of EXAFS, SEXAFS and XANES, volume 92 of Chemical Analysis (Wiley-Interscience, New York, 1988).

[118] T. Mayer-Kuckuk, Kernphysik (Teubner, Stuttgart, 1994).

[119] K.V. Klementev, www.desy.de/ klmn/viper.html, DESY, Hamburg, 2000, VIPER-Programm.

[120] K.V. Klementev, J. Phys. D 34 (2001) 209.

[121] S. Nasu, Hyp. Int. 128 (2000) 101.

[122] M. Uhrmacher et al., Nucl. Instr. and Meth. B 9 (1985) 234.

[123] W.K. Chu, J.W. Mayer and M.A. Nicolet, Backscattering Spectrometry (Academic Press, Orlando, 1978).

[124] W.H. Bragg and R. Kleeman, Philos. Mag. 10 (1905) 318.

[125] A. Wachter and H. Hoeber, Repetitorium Theoretische Physik (Springer, Berlin, Heidelberg, 2005).

[126] H. Frauenfelder and E.M. Henley, Teilchen und Kerne (Oldenbourg, München, Wien, 1995).

[127] N. Bohr, Mat. Phys. Medd. Dan. Vid. Selsk. 18 (1948) 8.

[128] J. Lindhard and M. Scharff, Mat. Phys. Medd. Dan. Vid. Selsk. 27 (1953) 15.

[129] H.H. Behncke, Härterei-Technische Mitteilungen 48 (1993) 3.

[130] H.H. Behncke and W. Weiler, Materialprüfung (Sonderdruck) Heft 7-8 (1988).

[131] P. Schaaf, Prog. Mat. Sci. 47 (2002) 1.

[132] P. Neumaier and H. Fischer, I-Lack 60. Jahrgang (1992) 375.

[133] P.S. Prévey, Metals Handbook (American Society for Metals (ASM), Metals Park, Ohio, 1986).

[134] S. Krumm, www.geol.uni-erlangen, Universität Erlangen, 1997, WinFit-Programm.

[135] R. Allmann, Röntgen-Pulverdiffraktometrie (Springer-Verlag, Berlin, Heidelberg, New York, 2002).

[136] R.A. Young, The Rietveld Method (Oxford University Press, Oxford, 1993).

[137] E. Jäger and R. Perthel, Magnetische Eigenschaften von Festkörpern (Akademie Verlag, Berlin, 1996).

[138] A. Hubert and R. Jäger, Magnetic Domains (Springer Verlag, Berlin, Heidelberg, New York, 1998).

[139] K. Zhang, Dissertation, Universität Göttingen, 2001.

[140] W. Demtröder, Molekülphysik (Oldenbourg, München, Wien, 2003).

[141] C.N. Banwell and E.M. McCash, Molekülspektroskopie (Oldenbourg, München, Wien, 1999).

[142] American Rolling Mill Company (ARMCO), http://www.aksteel.de, AK Steel GmbH, Köln. 
[143] Goodfellow Cambridge Limited, http://www.goodfellow.com/csp/active/gfHome.csp, Huntingdon, England.

[144] C. Illgner, Dissertation, Universität Göttingen, 1996.

[145] E. Mazur, Interaction of Ultrashort Laser Pulses with Solids, Spectroscopy and Dynamics of Collective Excitations in Solids, edited by B.D. Bartolo, p. 417, Plenum Press, New York, 1997.

[146] W.W. Duley, UV-Lasers: Effects and Applications in Materials Science (Cambridge University Press, Cambridge, 1996).

[147] W. Demtröder, Laserspektroskopie (Springer-Verlag, Berlin, Heidelberg, 2000).

[148] K.H. Hellwege and O. Madelung, editors, Landolt-Börnstein, volume 15(b) of Neue Serie III (Springer, Berlin, 1985).

[149] Y.S. Touloukian et al., Thermal diffusivity, Thermophysical Properties of Matter, edited by Y.S. Touloukian and C.Y. Ho, volume 10 of The TPRC Data Series, Plenum, New York, Washington, 1973.

[150] V. Semak and A. Matsunawa, J. Phys. D 30 (1997) 2541.

[151] R.K. Singh and J. Narayan, Mat. Sci. Eng. B 3 (1989) 217.

[152] H.S. Carslaw and J.C. Jaeger, Conduction of Heat in Solids (Clarendon Press, Oxford, 1959).

[153] T. Kasuya and N. Shimoda, J. Appl. Phys. 82 (1997) 3672.

[154] S.I. Anisimov and V.A. Khokhlov, Instabilities in Laser-Matter Interaction (CRC Press, Boca Raton, London, Tokyo, 1995).

[155] S. Sankaranarayana and A. Kar, J. Phys. D 32 (1999) 777.

[156] S. Sankaranarayana, H. Emminger and A. Kar, J. Phys. D 32 (1999) 1605.

[157] C.L. Chan and J. Mazumder, J. Appl. Phys. 62 (1987) 4579.

[158] S. Fähler and H.U. Krebs, Appl. Surf. Sci. 96-98 (1996) 61.

[159] J.M. Poate and J.W. Mayer, Laser Annealing of Semiconductors (Academic Press, New York, London, 1982).

[160] S.U. Campisano, Appl Phys. A 30 (1983) 195.

[161] M. Bollhöfer and V. Mehrmann, Numerische Mathematik (Vieweg, Wiesbaden, 2004).

[162] C.A.J. Fletcher, Computational Techniques for Fluid Dynamic, volume 1 (Springer, Berlin, 1988).

[163] F. Landry, Dissertation, Universität Göttingen, 1999.

[164] D.I. Rosen et al., J. Appl. Phys. 53 (1982) 3190.

[165] D.I. Rosen, D.E. Hastings and G.M. Weyl, J. Appl. Phys. 53 (1982) 5882.

[166] W.H. Kegel, Plasmaphysik (Springer-Verlag, Berlin, Heidelberg, 1998).

[167] M.H. Glowacki, J. Phys. D 28 (1995) 2051.

[168] R.K. Singh and J. Narayan, Phys. Rev. B 41 (1990) 8843.

[169] N. Arnold, J. Gruber and J. Heitz, Appl. Phys. A 69 [Suppl.] (1999) S87.

[170] R.G. Root, Modeling of Post-Breakdown Phenomena, Laser-Induced Plasmas and Applications, edited by L.J. Radziemski and D.A. Cremers, p. 69, Marcel Dekker Inc., New York and Basel, 1992. 
[171] A.N. Pirri, Phys. Fluids 16 (1973) 1435.

[172] A.N. Pirri, R. Root and P.K.S. Wu, AAIA Journal 16 (1978) 1296.

[173] Y.P. Răzer, Sov. Phys. JETP 31 (1970) 1148.

[174] Y.P. Raŭzer, Sov. Phys. JETP 21 (1965) 1009.

[175] A.A. Boni and F.Y. Su, Phys. Fluids 17 (1974) 340.

[176] N. Ferriter et al., AIAA Journal 15 (1977) 1597.

[177] M. Han, Dissertation, Universität Göttingen, 2001.

[178] Y.B. Zel'dovich and Y.P. Raŭzer, Physics of Shock Waves and High Temperature Phenomena (Academic Press, New York, London, 1966).

[179] W. Wagner and K.M. de Reuck, Methane - International Thermodynamic Tables of the Fluid State, volume 13 (No. 41) of IUPAC Chemical Data Series (Blackwell Science, Oxford, 1996).

[180] Air Liquide, Zentrale Düsseldorf, Gasekatalog, http://www.airliquide.de/loesungen/produkte/gase/ gasekatalog/stoffe/methan.html.

[181] Y.P. Ră̌zer, Sov. Phys. Quantum Electron. 14 (1984) 40.

[182] J.P. Reilly, A. Ballantyne and J.A. Woodroffe, AAIA Journal 17 (1979) 1098.

[183] G. Herziger and E.W. Kreutz, Phys. Scripta T 13 (1986) 139.

[184] V.Y. Balandin, D. Otte and O. Bostanjoglo, J. Appl. Phys. 78 (1995) 2037.

[185] T. Iida and R.I.L. Guthrie, The Physical Properties of Liquid Metals (Clarendon Press, Oxford, 1988).

[186] A.D. Zweig, J. Appl. Phys. 70 (1991) 1684.

[187] M. van Allmen, J. Appl. Phys. 47 (1976) 5460.

[188] T. Yoshida and K. Akashi, J. Appl. Phys. 48 (1977) 2252.

[189] L.R. Doolittle, Nucl. Instrum. Methods B 9 (1985) 344.

[190] N. Barrades, R. Webb and C. Jeynes, editors, The IBM DataFurnace WiNDF Version 7.0.x (University of Surrey, Guildford UK, 2001).

[191] J. Foct et al., J. Physique 40, Coll. C2, Suppl. 3 (1979) C2.

[192] A.C. Ferrari and J. Robertson, Phys. Rev. B 61 (2000) 14095.

[193] A.C. Ferrari and J. Robertson, Phys. Rev. B 64 (2001) 075414.

[194] S. Prawer et al., Diamond Relat. Mater. 5 (1996) 433.

[195] B. Jönsson and S. Hogmark, Thin Solid Films 114 (1984) 257.

[196] V.G. Gavriljuk, B.D. Shanina and H. Berns, Acta Mater. 48 (2000) 3879.

[197] V.M. Nadutov, Mat. Sci. Eng. A254 (1998) 234.

[198] P. Schaaf, S. Wiesen and U. Gonser, Acta Metall. Mater. 40 (1992) 373.

[199] P. Schaaf et al., Acta Metall. Mater. 42 (1994) 3077.

[200] G. Bertotti, Hysteresis in Magnetism (Academic Press, San Diego, London, 1998). 
[201] B.L. Henke, E.M. Gullikson and J.C. Davis, Atomic Data and Nucl. Data Tables 54 (1993) 181.

[202] W.T. Elam et al., Phys. Rev. B 38 (1988) 26.

[203] B.K. Teo and P.A. Lee, J. Am. Chem. Soc. 101 (1979) 2815.

[204] A.L. Ankudinov et al., Phys. Rev. B 58 (1998) 7565.

[205] J.J. Rehr and R.C. Albers, Rev. Mod. Phys. 72 (2000) 621.

[206] B. Ravel, J. Synchrotron Rad. 8 (2001) 314.

[207] T. Moriya et al., J. Phys. Soc. Jpn. 24 (1968) 60.

[208] H. Ino, S. Nasu and U. Gonser, J. de Physique 40 (1980) C1.

[209] H. Ino et al., Acta Met. 30 (1982) 9.

[210] J.P. Eymery et al., Thin Solid Films 217 (1992) 1.

[211] B. Boubeker et al., Nucl. Inst. Meth. B 101 (1995) 267.

[212] P. Plessis, P. Marmet and R. Dutil, J. Phys. B 16 (1983) 1283.

[213] V. Mazhukin, I. Smurov and G. Flamant, J. Comput. Phys. 112 (1994) 78.

[214] I. Schmidt and E. Hornbogen, Z. Metallkde. 69 (1978) 221.

[215] M.A. Otooni, Elements of Rapid Solidification: Fundamentals and Applications, volume 29 of Springer Series in Materials Science (Springer, Berlin, Heidelberg, New York, 1998).

[216] M. von Allmen, Laser Quenching, Glassy Metals II, edited by H. Beck and H.J. Güntherodt, volume 53 of Topics in Applied Physics, Springer, Berlin, Heidelberg, New York, Tokyo, 1983.

[217] T. Egami, Mat. Sci. Eng. A 226 (1997) 261.

[218] D.E. Polk, Acta Met. 20 (1972) 485.

[219] C.H. Bennett, D.E. Polk and D. Turnbull, Acta Met. 19 (1971) 1295.

[220] J.F. Shackelford and W. Alexander, The CRC Materials Science and Engineering Handbook, 3 ed. (CRC Press, Boca Raton, 2001).

[221] T.B. Massalski, Relationships between the Glass-forming Ability and several Metallurgical Parameters, Rapidly Quenched Metals, edited by S. Steeb and H. Warlimont, volume 1, North-Holland, Amsterdam, Oxford, New York, Tokyo, 1985.

[222] E. Thune and T.C.M. Jaouen, Phys. Rev. B 68 (2003) 115434. 


\section{Danksagung}

...eigentlich ist es überflüssig zu erwähnen, dass alle Mitarbeiterinnen und Mitarbeiter des II. Physikalischen Instituts sowohl zur überaus freundlichen Atmosphäre beigetragen als auch in irgendeiner Weise mit Rat und Tat zur Seite gestanden haben, wenn immer ich Hilfe brauchte. Allen gebührt mein größter Dank, sei es in wissenschaftlichen, elektronischen, mechanischen, PC- oder in verwaltungstechnischen Angelegenheiten.

Mir wäre es am liebsten, hier entweder alle oder keine Personen aufzuzählen. Ganz ohne geht es leider nicht, daher versuche ich, es kurz und bündig zu halten:

In erster Linie danke ich natürlich Herrn Prof. Dr. P. Schaaf für das Interesse an der Arbeit, die Unterstützung und das Bereitstellen des Themas.

Den ehemaligen Mitarbeitern Herrn Dr. G.A. Müller, Herrn Dr. M. Schwickert und Herrn Dr. E. Carpene für die Einführung in die verschiedenen Messmethoden und Datenauswertungs-Techniken.

Stellvertretend für die Feinmechanik-Werkstatt Herrn K. Arndt und für die Elektronik-Werkstatt Herrn K. Schemmerling.

Herrn D. Purschke und Herrn A. König für die Einführung und Bedienung des IONAS.

Herrn Dipl.-Phys. J. Zennek für die Raman-Messungen.

Herrn Dr. K. Zhang für die MOKE-Messungen.

Frau A. Traverse am L.U.R.E. in Orsay/Paris und Frau S. Ramos am E.S.R.F in Grenoble für die EXAFSMessungen.

...und last but not least danke ich den übrigen Diplomandinnen und Diplomanden bzw. Doktorandinnen und Doktoranden des II. Insitutes für die sehr schöne und fröhliche Zeit, für die Grillabende, Feiern, Seminare, Praktika, fruchtbaren und weniger ernsten Diskussionen. 



\section{Lebenslauf}

\section{Persönliche Daten:}

Name:

Michael Kahle

Geburtsdatum:

20. August 1973

Geburtsort:

Göttingen, Deutschland

Staatsangehörigkeit:

deutsch

Familienstand: ledig

\section{Schulausbildung:}

1980-1984

Adolf-Reichwein-Grundschule in Göttingen

$1984-1986$

Bert-Brecht-Orientierungsstufe in Göttingen

1986-1993

Hainberg-Gymnasium in Göttingen,

Abitur: 12.05.1993

Wehrdienst:

07/1993-06/1994

Zwölfmonatiger Grundwehrdienst in Munster

\section{Hochschulausbildung:}

WS 1994/95-WS 2000/01: Physikstudium an der Georg-August-Universität zu Göttingen

17.10 .1996

10.11 .2000

11.01 .2006

\section{Arbeitserfahrung:}

$11 / 2000-02 / 2001$

$11 / 2001-02 / 2002$

ab 01.03 .2002
Vordipolm

Diplom in Physik:

Thema: „Breitbandige Ultraschallspektroskopie

an Vesikel-Suspensionen von Lecithinen"

am III. Physikalischen Institut

Abschluss der Promotion:

Thema: „Laser-Carburisierung von Eisen und Edelstahl“

HiWi am III. Physikalischen Institut

HiWi am II. Physikalischen Institut

Wissenschaftlicher Angestellter am II. Physikalischen Institut 